\title{
Auger decay in double core ionized molecules
}

\author{
Dissertation zur Erlangung des \\ mathematisch-naturwissenschaftlichen Doktorgrades \\ "Doctor rerum naturalium" \\ der Georg-August-Universität Göttingen im Promotionsprogramm \\ PROPHYS \\ der Georg-August University School of Science (GAUSS) \\ vorgelegt von \\ Ludger Inhester \\ aus Göttingen
}

Göttingen, 2013 


\section{Betreuungsausschuss:}

Prof. Dr. Helmut Grubmüller, Abteilung für theoretisches und computergestützte Biophysik, Max-Planck Institut für biophysikalische Chemie

Prof. Dr. Jörg Enderlein, Abteilung für Biophysik / komplexe Systeme, Drittes Physikalisches Institut, Georg-August Universität Göttingen

\section{Mitglieder der Prüfungskomission:}

Referent:

Prof. Dr. Helmut Grubmüller, Abteilung für theoretische und computergestützte Biophysik, Max-Planck Institut für biophysikalische Chemie

Koreferent:

Prof. Dr. Jörg Enderlein, Abteilung für Biophysik / komplexe Systeme, Drittes Physikalisches Institut, Georg-August Universität Göttingen

\section{Weitere Mitglieder der Prüfungskomission:}

Dr. Gerrit Groenhof, Computational biomolecular chemistry group, Nanoscience Center, Universität Jyväskylä

Prof. Dr. Simone Techert, AG Strukturdynamik (bio)chemischer Systeme, Max-Planck Institut für biophysikalische Chemie

Prof. Dr. Claus Ropers, AG Nano-Optik und Ultraschnelle Dynamik, Institut für Materialphysik, Georg-August Universität Göttingen

Prof. Dr. Sarah Köster, Nanoscale imaging of cellular dynamics group, Institut für Röntgenphysik, Georg-August Universität Göttingen

\section{Tag der mündlichen Prüfung:}




\begin{abstract}
X-ray free electron lasers allow to create and probe double core holes in molecules via successive ionization in considerable amount. The properties of these double core ionized states are in particular relevant for the radiation damage in X-ray coherent diffractive imaging (CDI) experiments with single molecules. In this thesis the Auger decay of double core ionized states in small molecules is investigated via quantum chemical ab-initio methods. To model the emitted Auger electrons at continuous energy levels the single center method is used, in which the electronic wave function is described on a radial grid using spherical harmonics. As shown for the example of a water molecule, the proton dynamics induced by the double core ionization is reflected in the Auger spectrum by marked tails on the high-energy part of each spectral peak. The life time of double core holes in molecules is significantly reduced compared to that of single core holes due to the core hole induced screening effects of the valence electrons. This mechanism is explained by a simple model from which a relation for the decay rate and valence electron population is derived. Possible consequences of these results for X-ray diffraction experiments are: First, even for pulses shorter than 10fs the diffraction patterns is biased by the core hole induced rearrangement of the electronic valence structure. Second, the overall ionization rate is enhanced because of the faster refilling of double core holes.
\end{abstract}




\section{Zusammenfassung}

Röntgen Freie Elektronen Laser ermöglichen es Doppel-K-Schalen Löchern in Molekülen in aufeinanderfolgenden mehrfachen Ionisationsschritten in bedeutender Anzahl zu erzeugen. Die Eigenschaften dieser zweifach ionisierten Zustände ist insbesondere relevant für die Strahlungsschäden bei Beugungsexperimenten mit kohärenter Röntgenstrahlung zur Bildgebung einzelner Moleküle. In dieser Arbeit wird der Auger Zerfall doppelt K-Schalen ionisierter Moleküle mittels quantenchemischer ab-initio Methoden untersucht. Zur Beschreibung des emittierten Auger Elektrons im kontinuierlichen Energiespektrum wird dabei die Ein-Zentrums Methode verwendet, in der die elektronische Wellenfunktion auf einem radialen Gitter beschrieben wird unter Verwendung von sphärischen Harmonischen. Wie anhand des Wassermoleküls gezeigt wird, ergeben sich durch die DoppelK-Loch induzierte Protonendynamik in dem Auger Spektrum ausgeprägte Flanken im höherenergetischen Teil jeder Spektralspitze. Die Lebensdauer von Doppel-K-Schalen Löchern in Molekülen ist deutlich verringert im Vergleich zu einfachen K-Löchern durch die K-Loch induzierten Abschirmeffekte der Valenzelektronen. Dieser Mechanismus wird durch ein einfaches Modell erklärt aus dem eine Beziehung zwischen Zerfallsrate und Valenzelektronenpopulation abgeleitet. Mögliche Konsequenzen dieser Ergebnisse für Röntgenbeugungsexperimente sind: Erstens, auch für Röntgenpulse kürzer als 10fs wird das Beugungsbild durch die K-Loch induzierten Umstrukturierungen der Valenzelektro-

nen beeinflußt. Zweitens, die Gesamt-Ionisationsrate ist erhöht aufgrund der schnelleren Neubesetzung der K-Löcher. 


\section{Contents}

Contents

$\begin{array}{lll}1 & \text { Introduction } & 1\end{array}$

\begin{tabular}{|lll}
\hline 2 & Theory and numerical implementation & 7
\end{tabular}

2.1 Electronic structure calculation $\ldots \ldots \ldots \ldots$. . . . . . . . 8

$2.1 .1 \quad$ Born-Oppenheimer approximation $\ldots \ldots \ldots$. . . . . . . . 8

2.1 .2 Hartree-Fock approximation $\ldots \ldots \ldots \ldots$

$2.1 .3 \quad$ Configuration interaction $\ldots \ldots \ldots \ldots \ldots$

$2.2 \quad$ X-ray matter interaction $\ldots \ldots \ldots \ldots \ldots \ldots$

2.2 .1 The electromagnetic field $\ldots \ldots \ldots \ldots$

2.2 .2 Molecule-field interaction . . . . . . . . . . . . . . 17

$2.2 .3 \quad$ Derivation of the Pauli master equation $\ldots \ldots . \ldots 20$

2.3 Single center method $\ldots \ldots \ldots \ldots$. . . . . . . . . . . . . . . . . . . . . . 34

$2.3 .1 \quad$ Expansion in spherical harmonics . . . . . . . . . . . . . . . 34

$2.3 .2 \quad$ Asymptotics of the continuum electron wave function . . . . . . 38

$2.4 \quad$ Numerical procedure for solving the continuum wave function . . . . . . . 42

2.4 .1 Grid points . . . . . . . . . . . . . . . . . . . . . 42

2.4 .2 Vector sweep integration for Eq. $|2.126|$ with given $\mathbf{X}$. . . . . . . . 43 


\section{CONTENTS}

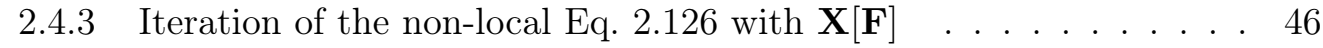

2.4 .4 Normalization . . . . . . . . . . . . . . . . . . . . . . . . . . . 48

2.4 .5 Radial truncation . . . . . . . . . . . . . . . . . . . . . . 48

2.4 .6 Truncation of angular quantum numbers $\ldots \ldots \ldots$. . . . . . . 48

$2.4 .7 \quad$ Exchange potential approximation . . . . . . . . . . . . . . 49

2.4 .8 Calculation of transition amplitudes $\ldots \ldots \ldots$

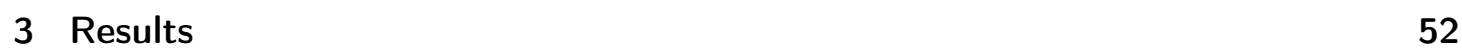

3.1 Auger decay in neon $\ldots \ldots \ldots \ldots$. . . . . . . . . . . . . . . . . 52

3.1.1 Convergence of initial and final state energies with respect to basis sets and CI truncation . . . . . . . . . . . . . . . . . 52

3.1.2 Convergence of transition rates with respect to basis set and CI truncation . . . . . . . . . . . . . . . . . . 56

3.1.3 Auger spectrum of neon after single and double core ionization . . 60

3.1 .4 Discussion . . . . . . . . . . . . . . . . . . . . . . . . . 63

3.2 Auger spectrum of water after single and double core ionization . . . . . 63

$3.2 .1 \quad$ Introduction $\ldots \ldots \ldots \ldots \ldots$. . . . . . . . . . . . . . . . . . .

3.2 .2 Article $\ldots \ldots \ldots \ldots \ldots \ldots$

$3.3 \quad$ Double core hole decay and core hole screening in first row hydrides . . . 77

3.3 .1 Introduction . . . . . . . . . . . . . . . . . . . . . . . 77

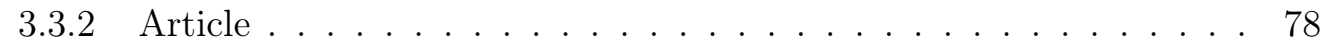

$3.4 \quad$ Auger spectrum of acetylene after single and double core ionization . . . . 83

$3.4 .1 \quad$ Introduction $\ldots \ldots \ldots \ldots$. . . . . . . . . . . . . . . . . . . . . . . 83

3.4 .2 Computational details $\ldots \ldots \ldots \ldots \ldots$

$3.4 .3 \quad$ Auger spectrum after single core ionization (K-LL) $\ldots . \ldots .87$ 
CONTENTS

$3.4 .4 \quad$ Auger spectrum after double core ionization (KK-KLL) . . . . . . 90

3.4 .5 Discussion \& Outlook . . . . . . . . . . . . . . . . . . . . 97

4 Conclusion \& Outlook 98 


\section{Acknowledgments}

I want to thank my supervisors Helmut Grubmüller, Jörg Enderlein, and Gerrit Groenhof for the steady support during this Phd project. Furthermore, I would like to express my gratitude to the additional members of the examination committee, Sarah Köster, Claus Ropers, and Simone Techert. For careful reading of drafts and helpful comments I am indebted to Carl F. Burmeister, Jan Braun, Maike Clemens, Hossein Ebadi, Georgia Erdmann, Konstantin Finke, Petra Kellers, Timo Graen, Gerrit Groenhof, Helmut Grubmüller, and Bernd Inhester. For the many fruitfull discussions, a special thanks goes to my colleagues at the MPI, in particular Carl F. Burmeister, Maike Clemens, Mehdi Davari, Hossein Ebadi, Zhong Yin, and the whole department of theoretical and computational biophysics. 


\section{Introduction}

Since the first experiments by Friedrich et al. [35], X-ray crystallography has become an established and essential tool for investigating the structure of matter. The seminal reconstruction of nucleic acids by Watson et al. 99] and the structure determination of myoglobin by Kendrew et al. 56 are two of the milestones for applying this technique on biological samples. Today, X-ray crystallography is the most important method for structure determination of biological macromolecules [31, 78]. The major limitation of this method is, however, the necessity of prior crystallization of the sample. The newly available XFEL (X-ray free electron lasers) holds the promise to overcome this drawback. One of the perspective applications of XFELs are coherent diffractive imaging (CDI) experiments with single non-crystalline samples [37, 71]. A primary goal of this concept is the determination of the structure of bio-molecules in atomic resolution, without prior crystallization. In the field of structural biology, this technique would be of high relevance, as it would give insight into the structure of macromolecules such as certain membrane proteins which cannot be crystallized.

According to the concept of CDI, a sample is exposed to an intense X-ray pulse with large spatial coherence. At the high intensity provided by XFELs the recording of the elastically scattered X-ray radiation enables the reconstruction of the electron density distribution in the sample and thus allows the investigation of the structure of the sample in atomic resolution. However, when exposed to this large amount of ionizing radiation, a bio-molecule will suffer from severe radiation damage and it will eventually loose its structural integrity in a Coulomb explosion. To circumvent this problem, the X-ray pulse has to be short enough, such that diffraction at the molecule occurs before the relevant structure of the molecule is destroyed. Further, many diffraction patterns of identical samples have to be accumulated to yield sufficient scattering data. This concept, known as diffract and destroy strategy, is illustrated in Fig. 1.1.

For nano-crystallized biological samples, diffract and destroy experiments with ultrashort X-ray pulses from a XFEL source have been successfully performed recently 18 , 67, 76]. The success of this method relies on the mechanism of self-terminating diffraction [6]. As soon as the crystalline order is lost as a consequence of radiation damage, 


\section{Introduction}

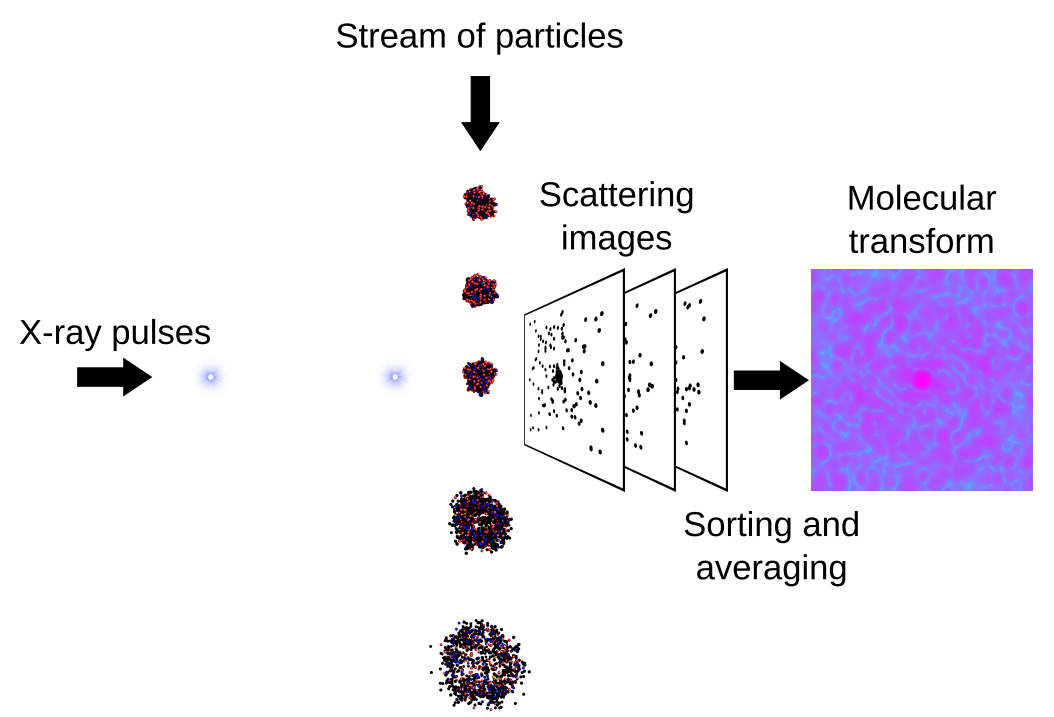

Figure 1.1: Illustration of the diffract and destroy strategy: A beam of identical samples is hit with the X-ray beam. The diffraction patterns are recorded, while the sample is destroyed. The collected diffraction patterns are classified for the different orientations, from which the sample structure is then reconstructed. Figure taken from Ref. 15.

the coherent Bragg diffraction signal from the crystal decreases. The damaged molecules do not produce their own specific diffraction pattern, so that their homogeneous background signal can be separated from the Bragg diffraction of the intact molecules. By accumulating several diffraction patterns of identical samples in sequential shots, it was possible to maintain a sufficient signal and reconstruct the intact molecular structure in atomic resolution.

To investigate under which circumstances this principle could also work for noncrystalline, single molecule samples is crucial. An important parameter in this context is the timescale at which the molecule dissociates under the influence of the heavy ionizing radiation. It is well known from experiments and atomic calculations, that the primary ionization events due to X-ray radiation are core shell photo-ionizations. For the biologically most relevant elements carbon $(\mathrm{C})$, nitrogen $(\mathrm{N})$, and oxygen $(\mathrm{O})$ core shell ionization contributes approximately 90 to $95 \%$ to the total ionization cross section at photon energies of $\simeq 8 \mathrm{keV}$ [101]. At the same photon energy, the elastic scattering cross section, which is responsible for the formation of the diffraction image, is about ten times smaller than that for ionization [7]. After ionization, core vacancies in the $\mathrm{C}, \mathrm{N}$, and $\mathrm{O}$ atom shells are refilled via Auger decay or fluorescence with a branching ratio of 


\section{Introduction}

approximately $99 \%$ for the Auger decay [60].

Recently, it has been demonstrated that the XFELs are capable to also produce double $\mathrm{K}$-shell vacancies in significant amounts $25,29,48,80,102$. The creation of these highly excited electronic states is possible because the ionization rate is of the same magnitude as the decay rate of the K-vacancies, such that there is a decent probability that the second electron is stripped off the K-shell before the first $\mathrm{K}$-vacancy is refilled. With an empty K-shell, the core vacant electronic states have a strongly reduced ionization cross section, such that the respective atom becomes temporarily nearly transparent to X-ray radiation - a mechanism known as frustrated absorption [48, 102]. Electronic states with empty K-shell are not only interesting on its own $8,17,25,61,62,90$, but also open up the question, whether the induced transparency of double K-holes might effectively delay the radiation damage in a CDI experiment 68.

Whereas the radiation damage mechanisms of XFEL radiation for atoms are fairly well understood, much higher complexity for molecular or atomic cluster samples is expected. First estimates of the structural disintegration process of a bio-molecule have been made based on molecular dynamics simulations [71]. In these simulations, nuclei were propagated classically under the influence of force fields describing chemical bonds. Additionally, Coulomb forces mimic the rapid charging of the molecule via photo-ionization and Auger decay processes. The investigated atomic displacements and the simulated diffraction patterns computed in this study suggest that a structure determination via CDI in atomic resolution might be possible for X-ray pulse durations shorter than a few femtoseconds. Later studies $41,45,46,52,54,104$ also modeled the dynamics of released photo- and Auger electrons. These studies revealed that due to the high ionization levels, the emitted electrons become trapped within the sample by the appreciable Coulomb force and screen the high charge in the center of the sample. The screening by these quasi unbound electrons delays the dissociation of the inner core of the sample such that the destruction of the sample proceeds much faster in the outer boundary. As a consequence, it has been suggested that a bio-molecule covered with a layer of, e.g. water, might resist XFEL radiation substantially longer [46].

The validity of above simulations relies on the photo ionization cross sections and Auger decay rates which determine the speed at which the sample charges up. The cross sections and Auger decay rates used are mostly estimated from atomic calculations and - due to the complexity of the problem - only few attempts have been made to investigate ionization and Auger decay involving highly ionized states in molecular calculations [58] or atomic calculations in an embedded charged environment [4, 94]. Other relevant molecular effects like the migration of electron density, which directly changes 


\section{Introduction}

the diffraction pattern and indirectly might influence the further atomic motion as well as subsequent ionization processes were not modeled in these simulations. Recent experiments [30, 33] with small molecules revealed the relevance of these processes, and call for a better understanding of the electronic radiation damage in molecules. A detailed investigation of electron dynamics and Auger decay in molecules after multiple ionization via quantum mechanical ab initio calculations would thus provide crucial insight into the effective radiation damage in CDI experiments and allows to give more reliable parameters to simulate the XFEL induced molecular dissociation.

Apart from the radiation damage in CDI experiments, the electronic structure of multiple core hole states in molecules also received considerable attention, because the spectroscopy of multiple hole states allows a more sensitive probing of the electronic structure than single core holes $8,17,61,62,90$. In particular, probing electronic states in which core holes are located at different nuclear sites may give insight into the geometry and the electronic structure of a molecule, because their spectroscopic properties sensitively depend on how far the two core holes in the molecule are spatially separated. Computational efforts which address molecular ionization and Auger decay spectra are an essential requirement to understand and interpret spectroscopic measurements of these multiple core ionized states. Thus, also from this perspective, ab inito studies of the Auger decay of multiple core ionized molecules are desirable.

Despite of the large demand for investigating these processes, only few computational studies have so far addressed the Auger decay in molecules and even fewer considered multiple core ionization. The major challenges in the computational description of Auger decay processes in molecules are:

1. The computational description of highly excited electronic states in molecules.

2. The accurate description of the delocalized continuum wave function and of the localized core electrons within the same framework.

The first challenge results from the strong correlation in the involved core hole states or multiple ionized valence states. To address these electronic states, the so called algebraic diagrammatic construction [59] or the configuration interaction method [2] has been used. The second challenge results from the fact that for Auger and photo-ionization processes an electron is promoted from a localized to a completely delocalized state. Localized electrons are described in common quantum chemistry approaches with basis sets of Gaussian functions. Previous approaches have represented both the localized bound and the delocalized continuum electrons in the vicinity of the molecules with Gaussian functions 


\section{Introduction}

using the so called Stieltjes imaging method [16]. In particular for the high energetic continuum, this technique requires an enormous amount of basis functions [58 which puts strong limitations on the calculation. In another approach, known as the single center method [27, 103], the continuum wave function is expanded in spherical harmonics on a radial grid. This latter approach offers an acceptable representation also of high energy continuum states, but its application is limited to small molecules with high symmetry due to the computational costs which arise for high electronic angular momentum. In more simplified approaches an explicit description of the continuum wave function is avoided. In the one-center approach [5, 23, 84], molecular Auger transitions are calculated using amplitudes estimated from atomic calculations. Furthermore, Auger spectra have been estimated based on a population analysis [69, 98] or based on statistical considerations [89], in which the weights of the final state wave function on components with two valence holes are used as line intensities. Although these methods have given qualitative agreement with experiments in several cases, the effect of the molecular continuum wave function cannot generally be neglected. In particular, for reliable calculations of absolute transition rates, an explicit description of molecular continuum wave functions has to be considered 23, 103.

To address the ionization and Auger decay processes in a non-linear, polyatomic molecule, I have developed in this thesis an ab initio quantum chemistry approach to calculate ionization cross sections and Auger decay rates. The developed procedure is based on the single center method [27, 103] for the description of the continuum electron and on the configuration interaction method for the bound electrons.

Using this procedure I investigated the electron dynamics induced by double K-shell ionization in small molecules within the short timescales relevant for CDI experiments. The calculations provide emission spectra of Auger electrons, which may help to interpret future experimental data. They also demonstrate the immediate impact of multiple ionization on the electronic structure and on the nuclei dynamics. Furthermore, absolute decay rates of single and double K-shell vacancies in small molecules were obtained. For the dependency of the core hole lifetimes on the chemical environment, a qualitative relation was derived, which provides essential parameters to model the radiation damage in CDI experiments with macromolecules.

\section{Outline of the thesis}

The outline of this thesis is as follows. Chapter 2 briefly introduces the theoretical methods, the underlying assumptions and limitations. Further, I discuss the numerical 


\section{Introduction}

implementation of the theoretical approach and give the details of the performed calculations. In the following chapter 3 , I present the obtained results. It contains two published articles, on which this cumulative thesis is based. The first article [50] studies the ionization dynamics and the Auger decay of a single water molecule exposed to high intense X-ray radiation. The second article [51 systematically investigates single and double K-hole lifetimes in first-row hydrides. Further results regarding Auger decay transitions in acetylene are presented at the end of chapter 3 . In chapter 4 I discuss the relevance of these results from a broader perspective and give an outlook on future work. 


\section{Theory and numerical implementation}

In this chapter, I introduce the theoretical models and the methods that were used for the calculations. To clarify the notation, the general methods for electronic structure calculations are briefly introduced in the first section. In the second section, concepts of X-ray-matter interaction are presented. By stepping through the derivation of the Pauli master equation starting from first principles, I illustrate the necessary approximations and discuss the conditions under which they are valid. Based on this derivation, the relevant X-ray-molecule interaction processes are discussed. Finally, I describe the concept of the single center method and the details of the numerical procedure I have used to obtain the electronic continuum wave function.

Throughout this thesis, atomic units (a.u.) are used. A list of fundamental constants in atomic and in standard units is given in Table 2.1. Table 2.2 lists the conversion of the most important units.

Table 2.1: Fundamental constants in standard (SI) and atomic units.

\begin{tabular}{l|c|c|l} 
Quantity & Symbol & SI value \& unit & a.u. value \\
\hline Planck constant divided by $2 \pi$ & $\hbar$ & $1.05 \cdot 10^{-34} \mathrm{~J} \cdot \mathrm{s}$ & 1 \\
elementary charge: & $e$ & $1.60 \cdot 10^{19} \mathrm{C}$ & 1 \\
electron mass: & $m_{e}$ & $9.11 \cdot 10^{-31} \mathrm{~kg}$ & 1 \\
Coulomb's constant: & $1 /\left(4 \pi \epsilon_{0}\right)$ & $8.987 \cdot 10^{9} \mathrm{Nm}^{2} / \mathrm{C}^{2}$ & 1 \\
speed of light: & $c$ & $299.8 \cdot 10^{6} \mathrm{~m} / \mathrm{s}$ & $1 / \alpha \simeq 137$
\end{tabular}

Table 2.2: Conversion of units.

\begin{tabular}{r|c|c} 
Quantity & Formula & SI conversion \\
\hline Length & $1 a_{0}:=4 \pi \epsilon_{0} \hbar^{2} /\left(m_{e} e^{2}\right)$ & 1 a.u. $=5.291 \cdot 10^{-11} \mathrm{~m}$ \\
Energy & $1 E_{\text {Hartree }}:=m_{e} e^{4} /\left(4 \pi \epsilon_{0} \hbar^{2}\right)$ & 1 a.u. \\
Time & $\hbar / E_{\text {Hartree }}$ & 1 a.u. $=211 \mathrm{eV}$ \\
Rate & $E_{\text {Hartree }} / \hbar$ & 1 a.u. $\hat{=} 4.14 \cdot 10^{-17} \mathrm{~s}$ \\
& &
\end{tabular}




\subsection{Electronic structure calculation}

In this section, I summarize the techniques for the electronic structure calculation used in this thesis, which are the Hartree-Fock approximation and the configuration interaction approach. I first introduce the Born-Oppenheimer approximation which yields a factorization of the molecular eigenstate into an electronic and a nuclear part. Then, I briefly review the main aspect of the Hartree-Fock approximation and the configuration interaction (CI) approach to obtain the many electron wave function.

\subsubsection{Born-Oppenheimer approximation}

The total Hamiltonian of a molecule is given in the non-relativistic limit by

$$
\begin{gathered}
H_{\mathrm{M}}=\sum_{n}^{\text {nuclei }}\left(\frac{P_{n}^{2}}{2 M_{n}}+\sum_{n \neq n^{\prime}}^{\text {nuclei }} \frac{Z_{n} Z_{n^{\prime}}}{\left|\mathbf{R}_{n}-\mathbf{R}_{n^{\prime}}\right|}\right) \\
+\sum_{i}^{\text {electrons }}\left(\frac{p_{i}^{2}}{2}+\frac{1}{2} \sum_{i^{\prime} \neq i}^{\text {electrons }} \frac{1}{\left|\mathbf{r}_{i}-\mathbf{r}_{i^{\prime}}\right|}-\sum_{n}^{\text {nuclei }} \frac{Z_{n}}{\left|\mathbf{r}_{i}-\mathbf{R}_{n}\right|}\right),
\end{gathered}
$$

where $\mathbf{R}_{n}$ denotes the position of the nuclei, which have charge $Z_{n}$ and mass $M_{n}, \mathbf{r}_{i}$ are the positions of the electrons, $\mathbf{P}_{n}$ and $\mathbf{p}_{n}$ are the nuclei and electron momenta, respectively.

The Born-Oppenheimer approximation assumes that, due to the much larger mass $M_{n}$, the nuclear motion can be neglected when considering the fast electronic motion, such that the total wave function can be written as a product of an electronic wave function $|\psi(\mathbf{R})\rangle$, which parametrically depends on all the nuclear coordinates $\mathbf{R}=\left(\mathbf{R}_{1}, \mathbf{R}_{2}, \ldots\right)$, and a nuclear wave function $|\chi\rangle$. The operators of electronic coordinates $\mathbf{r}_{i}$ and $\mathbf{p}_{i}$ only act on the electronic wave function $|\psi(\mathbf{R})\rangle$ so that the electronic eigenstate $\left|\psi_{j}(\mathbf{R})\right\rangle$ is defined by the electronic eigenvalue equation

$$
\begin{aligned}
& H_{\mathrm{el}}(\mathbf{R})\left|\psi_{j}(\mathbf{R})\right\rangle=\sum_{i}^{\text {electrons }}\left(\frac{\mathbf{p}_{i}^{2}}{2}\right.\left.+\frac{1}{2} \sum_{i^{\prime} \neq i}^{\text {electrons }} \frac{1}{\left|\mathbf{r}_{i}-\mathbf{r}_{i^{\prime}}\right|}-\sum_{n}^{\text {nuclei }} \frac{Z_{n}}{\left|\mathbf{r}_{i}-\mathbf{R}_{n}\right|}\right)\left|\psi_{j}(\mathbf{R})\right\rangle \\
&=E_{j}^{\mathrm{el}}(\mathbf{R})\left|\psi_{j}(\mathbf{R})\right\rangle
\end{aligned}
$$

The eigenvalue $E_{j}^{\mathrm{el}}(\mathbf{R})$ depends on the nuclear coordinates $\mathbf{R}$ and defines, together with 


\section{Theory and numerical implementation}

the nuclear-nuclear electrostatic term in Eq.2.1, the nuclear potential

$$
U_{j}(\mathbf{R}):=\sum_{n \neq n^{\prime}}^{\text {nuclei }} \frac{Z_{n} Z_{n^{\prime}}}{\left|\mathbf{R}_{n}-\mathbf{R}_{n^{\prime}}\right|}+E_{j}^{\mathrm{el}}(\mathbf{R})
$$

for the electronic state $j$. The remaining nuclear eigenvalue equation then reads

$$
H_{\text {nuc }}\left|\chi_{\nu}^{j}\right\rangle=\left(\sum_{n}^{\text {nuclei }} \frac{P_{n}^{2}}{2 M_{n}}+U_{j}(\mathbf{R})\left|\chi_{\nu}^{j}\right\rangle=E_{j, \nu}\right)\left|\chi_{\nu}^{j}\right\rangle .
$$

Thus, in the Born-Oppenheimer approximation, the eigenfunction of the total (nuclear and electronic) Hamiltonian, which depends on all nuclear coordinates $\mathbf{R}$ and all electronic coordinates $\mathbf{r}=\left(\mathbf{r}_{1}, \mathbf{r}_{2}, \ldots\right)$, is given by

$$
\Psi_{j, \nu}(\mathbf{r}, \mathbf{R})=\left\langle\mathbf{R}, \mathbf{r} \mid \Psi_{j, \nu}\right\rangle=\left\langle\mathbf{R} \mid \chi_{\nu}^{j}\right\rangle\left\langle\mathbf{r} \mid \psi_{j}(\mathbf{R})\right\rangle,
$$

where the index $j$ indicates the electronic eigenstate and the index $\nu$ denotes the molecular vibrational state, i.e. the nuclear eigenstate.

In the following two subsections, I consider the solution of the electronic Hamiltonian $H_{\mathrm{el}}(\mathbf{R})$ (Eq. 2.2). To improve the readability, I will drop the additional parameter $\mathbf{R}$ in the electronic Hamiltonian $H_{\mathrm{el}}$ and the electronic wave function $\left|\psi_{j}(\mathbf{R})\right\rangle$, keeping in mind that they both parametrically depend on the nuclear positions.

\subsubsection{Hartree-Fock approximation}

To solve the many particle problem, a popular approach in quantum chemistry is to approximate the many particle interaction by a mean field. This concept is known as the Hartree-Fock approximation, which I will now illustrate for the electronic Hamiltonian.

The electronic Hamiltonian $H_{\mathrm{el}}$ in Eq.2.2 for $N$ electrons reads

$$
H_{\mathrm{el}}=\sum_{i}^{N} \frac{p_{i}^{2}}{2}-\sum_{i}^{N} \sum_{n}^{\text {nuclei }} \frac{Z_{n}}{\left|\mathbf{r}_{i}-\mathbf{R}_{n}\right|}+\frac{1}{2} \sum_{i^{\prime} \neq i} \frac{1}{\left|\mathbf{r}_{i}-\mathbf{r}_{i^{\prime}}\right|}
$$

where the sum is taken over all $N$ electrons, $Z_{n}$ is the charge of the $n$-th nucleus at position $\mathbf{R}_{n}, \mathbf{p}_{i}$ and $\mathbf{r}_{i}$ is the momentum and position of electron $i$. Introducing a single particle basis $\phi_{\alpha}$, the electronic Hamiltonian is rewritten in second quantization 
as

$$
H_{\mathrm{el}}=\sum_{\alpha \beta}\langle\alpha|h| \beta\rangle c_{\alpha}^{\dagger} c_{\beta}+\frac{1}{2} \sum_{\alpha \beta \gamma \delta}\left\langle\alpha \beta\left|\frac{1}{r_{12}}\right| \gamma \delta\right\rangle c_{\alpha}^{\dagger} c_{\beta}^{\dagger} c_{\delta} c_{\gamma}
$$

where $c_{\alpha}^{\dagger}$ is a creation operator of an electron with one particle spin wave function $\left|\phi_{\alpha}\right\rangle$, satisfying the fermionic permutation rules

$$
\left[c_{\alpha}, c_{\beta}\right]=0, \quad\left[c_{\alpha}, c_{\beta}^{\dagger}\right]=\delta_{\alpha, \beta} .
$$

In Eq. 2.7, the one-particle interaction terms have been abbreviated as

$$
\langle\alpha|h| \beta\rangle:=\int d \mathbf{r} \phi_{\alpha}^{*}(\mathbf{r})\left(\frac{-\Delta}{2}-\sum_{A} \frac{Z_{A}}{\left|\mathbf{r}-\mathbf{R}_{A}\right|}\right) \phi_{\beta}(\mathbf{r}),
$$

and the two-particle Coulomb integral as

$$
\left\langle\alpha \beta\left|\frac{1}{r_{12}}\right| \gamma \delta\right\rangle:=\int d \mathbf{r}_{1} d \mathbf{r}_{2} \phi_{\alpha}^{*}\left(\mathbf{r}_{1}\right) \phi_{\beta}^{*}\left(\mathbf{r}_{2}\right) \frac{1}{\left|\mathbf{r}_{1}-\mathbf{r}_{2}\right|} \phi_{\gamma}\left(\mathbf{r}_{1}\right) \phi_{\delta}\left(\mathbf{r}_{2}\right) .
$$

Note that the basis functions $\phi$ are meant to include the spin degree of freedom, such that Eq. 2.9 is non-zero only when the spin quantum numbers of basis function $\phi_{\alpha}$ and $\phi_{\beta}$ are equal and, accordingly, Eq. 2.10 is non-zero only when the spin quantum numbers of basis function $\phi_{\alpha}$ and $\phi_{\gamma}$ as well as $\phi_{\beta}$ and $\phi_{\delta}$ are equal.

To introduce the Hartree-Fock mean field, the electronic Hamiltonian $H_{\mathrm{el}}$ is modified by approximating the two-electron terms in Eq. 2.7 by pairwise averaging over an electronic mean field state $|\mathrm{HF}\rangle[\mathbf{8 3}]$, i.e.

$$
\begin{aligned}
2 c_{\alpha}^{\dagger} c_{\beta}^{\dagger} c_{\delta} c_{\gamma} & \simeq c_{\alpha}^{\dagger} c_{\delta}\left\langle\mathrm{HF}\left|c_{\beta}^{\dagger} c_{\gamma}\right| \mathrm{HF}\right\rangle+c_{\beta}^{\dagger} c_{\gamma}\left\langle\mathrm{HF}\left|c_{\alpha}^{\dagger} c_{\delta}\right| \mathrm{HF}\right\rangle \\
& -c_{\alpha}^{\dagger} c_{\gamma}\left\langle\mathrm{HF}\left|c_{\beta}^{\dagger} c_{\delta}\right| \mathrm{HF}\right\rangle-c_{\beta}^{\dagger} c_{\delta}\left\langle\mathrm{HF}\left|c_{\alpha}^{\dagger} c_{\gamma}\right| \mathrm{HF}\right\rangle
\end{aligned}
$$

Inserting this approximation into the original Hamiltonian $H_{\mathrm{el}}$ yields the Hartree-Fock Hamiltonian

$$
H_{\mathrm{HF}}=\sum_{\alpha \beta}\left[\langle\alpha|h| \beta\rangle+\sum_{\gamma \delta} \frac{1}{2}\left(\left\langle\alpha \delta\left|\frac{1}{r_{12}}\right| \gamma \beta\right\rangle-\left\langle\alpha \delta\left|\frac{1}{r_{12}}\right| \beta \gamma\right\rangle\right)\left\langle\mathrm{HF}\left|c_{\delta}^{\dagger} c_{\gamma}\right| \mathrm{HF}\right\rangle\right] c_{\alpha}^{\dagger} c_{\beta},
$$

where the symmetry of the Coulomb integral $\left\langle\alpha \beta\left|\frac{1}{r_{12}}\right| \gamma \delta\right\rangle=\left\langle\beta \alpha\left|\frac{1}{r_{12}}\right| \delta \gamma\right\rangle$ has been exploited. The Hartree-Fock state $|\mathrm{HF}\rangle$, which is used for the mean field evaluation, solves 


\section{Theory and numerical implementation}

the eigenvalue problem

$$
H_{\mathrm{HF}}|\mathrm{HF}\rangle=E_{\mathrm{HF}}|\mathrm{HF}\rangle,
$$

self-consistently. Note that the Hartree-Fock state $|\mathrm{HF}\rangle$ does not necessarily need to be the ground state.

The reduction to an effective single particle interaction Hamiltonian allows to solve the eigenvalue problem for $N$ electrons by the single Slater determinant

$$
|\mathrm{HF}\rangle=c_{i_{N}}^{\dagger} \ldots c_{i_{2}}^{\dagger} c_{i_{1}}^{\dagger}|\mathrm{vac}\rangle
$$

where $|\mathrm{vac}\rangle$ is the vacuum state and $c_{i}^{\dagger}$ is the fermionic creation operator for an electron spin-orbital $\left\langle\mathbf{r} \mid \phi_{\mathbf{i}}\right\rangle$. The spin-orbitals $\left\langle\mathbf{r} \mid \phi_{i}\right\rangle$ are expanded in the one-particle basis by coefficients $C_{\alpha, i}$,

$$
\left|\phi_{i}\right\rangle:=\sum_{\alpha} C_{\alpha, i}\left|\phi_{\alpha}\right\rangle
$$

To determine the matrix $\mathbf{C}$ composed of the column vectors $\mathbf{C}_{i}$, the variational principle is used, that is, if $\mathbf{C}$ is chosen such that $|\mathrm{HF}\rangle$ solves Eq. 2.13 for an eigenvalue $E_{\mathrm{HF}}$, small variations on the energy expectation value for the varied solution $\mathbf{C}+\eta \mathbf{C}^{\prime}$ should vanish in first order of $\eta$, i.e.

$$
\frac{\partial}{\partial \eta} E\left\{\mathbf{C}+\eta \mathbf{C}^{\prime}\right\}:=\frac{\partial}{\partial \eta} \frac{\left\langle\operatorname{HF}\left\{\mathbf{C}+\eta \mathbf{C}^{\prime}\right\}\left|H_{\mathrm{HF}}\right| \operatorname{HF}\left\{\mathbf{C}+\eta \mathbf{C}^{\prime}\right\}\right\rangle}{\left\langle\operatorname{HF}\left\{\mathbf{C}+\eta \mathbf{C}^{\prime}\right\} \mid \operatorname{HF}\left\{\mathbf{C}+\eta \mathbf{C}^{\prime}\right\}\right\rangle}=0 .
$$

By additionally requiring the coefficient vectors $\mathbf{C}_{i}$, i.e. the column vectors of the matrix C, to be orthonormal,

$$
\sum_{\alpha} C_{i, \alpha} C_{\alpha, j}^{\dagger}=\delta_{i, j}
$$

one obtains in a few steps [87, pp. 108-129] the Hartree-Fock equation for the coefficient vectors $\mathbf{C}_{i}$,

$$
\sum_{\beta}\left(\langle\alpha|h| \beta\rangle+\sum_{\gamma, \delta}\left\langle\alpha \delta\left|\frac{1}{r_{12}}\right| \gamma \beta\right\rangle-\left\langle\alpha \delta\left|\frac{1}{r_{12}}\right| \beta \gamma\right\rangle \sum_{j}^{\mathrm{occ}} C_{j, \delta}^{\dagger} C_{\gamma, j}\right) C_{\beta, i}=\epsilon_{i} C_{\alpha, i},
$$

in compact notation written as

$$
\mathbf{F}[\mathbf{C}] \mathbf{C}_{i}=\epsilon_{i} \mathbf{C}_{i}
$$




\section{Theory and numerical implementation}

\section{Numerical solution of the Hartree-Fock equations}

Depending on the desired electronic state, different schemes of spin-restrictions for the coefficient vectors $\mathbf{C}_{i}$ were used in the calculations performed in this thesis. For closed shell states in which all electrons are spin-paired the spin symmetry was exploited by requiring that the coefficient vectors for the two spin populations are equal. This procedure is known as the restricted Hartree-Fock method (RHF). For open shell states with one or more unpaired electron spins, the unrestricted Hartree-Fock method (UHF) with no constraints on the coefficients was used. When the obtained molecular orbitals in the Hartee-Fock calculation were used as basis for later calculations (see the following subsection), restricted open shell Hartree-Fock calculations (ROHF) were performed, in which also for open shell states it is required that for both spin populations the coefficient vectors $\mathbf{C}_{i}$ are the same.

To numerically solve for the coefficient vectors $\mathbf{C}_{i}$ and orbital eigenvalues $\epsilon_{i}$ in Eq. 2.18, I used the Hartree-Fock procedure provided by the PSI3 [24] or the GAUSSIAN09 [36] quantum package. Basically, the solution for the coefficients $\mathbf{C}$ is found by iteratively solving the eigenvalue problem until the eigenvectors of the Fock Matrix $\mathbf{F}[\mathbf{C}]$ are consistent with the set of coefficient vectors $\mathbf{C}_{i}$ from which the Fock matrix has been calculated. Usually, these calculations converge to an approximate description of the electronic ground state for the respective number of electrons and spin assignment. To also obtain an approximate description for core ionized states, convergence to this desired state was achieved by choosing the initial guess for the coefficients $\mathbf{C}$ such that they are similar to the desired core hole state. This was accomplished by first performing a ground state calculation, and then use the obtained ground state result for the coefficient vectors $\mathbf{C}$ with rearranged electronic occupations as initial guess for the core ionized state calculation. In general, the convergence to the desired state is not guaranteed, but for vacancy states in orbitals with isolated orbital eigenvalues $\epsilon_{i}$, which were mostly addressed in this work, the desired vacancy state is usually obtained by the described procedure. The Hartree-Fock method therefore provides a computationally cheap tool for obtaining reliable estimates for core ionized states [61].

\subsubsection{Configuration interaction}

In the previous subsection, the solution to the electronic wave function was found as a single Slater determinant by introducing a mean field. Usually, the energy eigenvalues obtained by the Hartee-Fock approximation are relatively accurate, however relevant 


\section{Theory and numerical implementation}

properties of the electronic wave function are badly described. This failure of the HartreeFock approximation is related to the fact that the electronic state is not well described by a single Slater determinant but requires rather a linear combination of different Slater determinants. This is expressed by the observation that the motion of a single electron is not independent from the other electrons but is correlated to them. These correlations are addressed by the configuration interaction (CI) method 47, 87 and thus yields a more accurate description of electronic states. This method also provides a way to obtain excited electronic states more reliably than using the Hartree-Fock method as the electronic states are deduced from a linear equation, whereas in the Hartree-Fock method electronic states are deduced from a non-linear equation instead. The concept of the CI method is to use a general many particle description of the wave function, which is briefly summarized in this subsection.

A general many particle basis of $N$ electrons is constructed by a set of Slater determinants

$$
\left|\phi_{j_{1}, \sigma_{1}} \ldots \phi_{j_{N}, \sigma_{N}}\right\rangle:=c_{j_{N}, \sigma_{N}}^{\dagger} \ldots c_{j_{1}, \sigma_{1}}^{\dagger}|\mathrm{vac}\rangle .
$$

Here, $\mid$ vac $\rangle$ is the vacuum state and $c_{j, \sigma}^{\dagger}$ is the fermionic creation operator for an electron with the wave function $\phi_{j}(\mathbf{r})$ with spin $\sigma$. Using a set of $K$ orthonormal one-electron basis functions $\phi_{j}(\mathbf{r})$ an expansion of a general $N$-electron wave function is written as

$$
|\psi\rangle=\sum_{\left\{\left(j_{1}, \sigma_{1}, \ldots, j_{N}, \sigma_{N}\right)\right\}} C_{j_{1}, \sigma_{1}, \ldots, j_{N}, \sigma_{N}}\left|\phi_{j_{1}, \sigma_{1}} \ldots \phi_{j_{N}, \sigma_{N}}\right\rangle
$$

where the sum is taken over all $\left(\begin{array}{c}N \\ 2 K\end{array}\right)$ ordered $N$-tuples distributing the $N$ electrons over the set of $K$ one-electron basis functions with spin quantum number $\sigma= \pm 1 / 2$. Expanding the Hamiltonian in this basis, the coefficients $C_{j_{1}, \sigma_{1}, \ldots, j_{N}, \sigma_{N}}$ and the energy eigenvalue for a certain Hamiltonian-eigenstate are obtained by solving the respective $\left(\begin{array}{c}N \\ 2 K\end{array}\right)$ dimensional eigenvalue problem. Note that this procedure (also known as the Full CI expansion) is exact, provided that the set of one-electron basis functions $\phi_{j}(\mathbf{r})$ is complete.

For the calculations performed here, the set of one-electron basis functions was taken from the set of molecular orbitals previously obtained in an Hartree-Fock calculation. 


\section{Theory and numerical implementation}

The involved Hamiltonian matrix elements

$$
\begin{gathered}
\left\langle\phi_{j_{1}^{\prime}, \sigma_{1}} \ldots \phi_{j_{N}^{\prime}, \sigma_{N}}\left|H_{e l}\right| \phi_{j_{1}, \sigma_{1}} \ldots \phi_{j_{N}, \sigma_{N}}\right\rangle \\
=\sum_{i, j}\langle i|h| j\rangle\left\langle\phi_{j_{1}^{\prime}, \sigma_{1}} \ldots \phi_{j_{N}^{\prime}, \sigma_{N}}\left|c_{i}^{\dagger} c_{j}\right| \phi_{j_{1}, \sigma_{1}} \ldots \phi_{j_{N}, \sigma_{N}}\right\rangle+ \\
\frac{1}{2} \sum_{i, j, k, l}\left\langle i j\left|\frac{1}{r_{12}}\right| l k\right\rangle\left\langle\phi_{j_{1}^{\prime}, \sigma_{1}} \ldots \phi_{j_{N}^{\prime}, \sigma_{N}}\left|c_{i}^{\dagger} c_{j}^{\dagger} c_{k} c_{l}\right| \phi_{j_{1}, \sigma_{1}} \ldots \phi_{j_{N}, \sigma_{N}}\right\rangle,
\end{gathered}
$$

were then calculated by using the respective one or two-particle interaction matrix elements $\langle i|h| j\rangle$ and $\left\langle i j\left|1 / r_{12}\right| l k\right\rangle$ provided by the PSI3 quantum package [24] with which the Hartree-Fock calculation was performed. The matrix elements for the creation and annihilation operators were evaluated using the so called Slater-Condon rules (see for example Ref. [87]). The Hamiltonian eigenstate was then found by using common linear algebra libraries 3,65 .

The sum in Eq. 2.21 can be dramatically reduced by contracting the Slater determinants to eigenfunctions of the total spin operators $S^{2}$ and $S_{z}$ with given spin quantum numbers $S$ and $M_{S}$ [73. These spin eigenstates form a new basis set, known as configuration state functions (CSFs). Similarly, if the molecule is symmetric, the configuration state functions can be contracted to satisfy a given spatial symmetry. By requiring the desired electronic state to fulfill this symmetry and spin properties, the dimension of the eigenvalue problem is strongly reduced.

However, exploiting spin and spatial symmetry does usually not suffice to reduce the number of multi-electron basis functions to a computationally feasible number. Thus, truncation schemes are used to have only the most relevant configuration state functions for a certain spin and spatial symmetry included in the expansion. For the calculations performed here, this was achieved by reducing the sum in Eq. 2.21 to CSFs close to a reference occupation or a set of reference occupations. Starting with a specific reference occupation (usually built from the MOs occupied as expected in the desired state), a set of configuration state functions was created by performing a given number of excitations, i.e. promotions of electrons from occupied MOs into unoccupied MOs. The procedure is illustrated in Fig. 2.1. The truncation schemes are in the following denoted as "CI0" for the CSFs drawn from the bare occupation reference, "CIS" for CI truncated to single excitations from the given reference, "CISD" for CI truncated to single and double excitations from the given reference, and so on. Also truncation schemes were used, where excitations from multiple references are taken into account. These truncation schemes are denoted in the following as "MRCIS", "MRCISD", etc. By increasing the number of excitations, the truncation error in the calculation can be systematically decreased and in this way the 
quality of the electronic structure calculation was assessed.
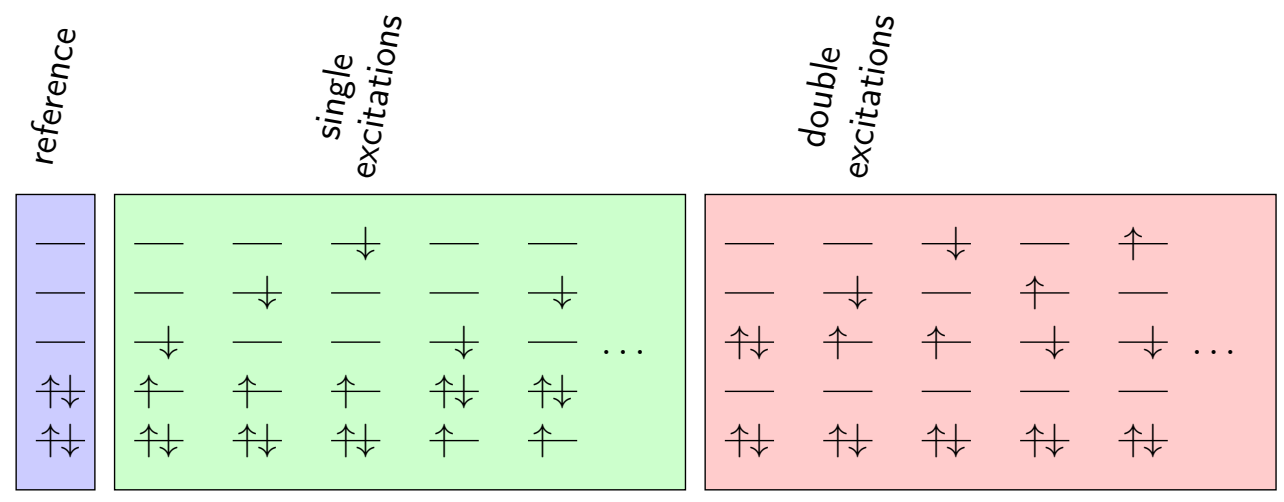

Figure 2.1: Illustration of truncation for CI expansion in terms of excitation levels. The blue box illustrates the reference state: four electrons occupying the lowest spin-orbitals. The green box illustrates all single excitations from the reference, where one electron has been promoted to orbitals which are unoccupied in the reference. The red box illustrates double excitations, where two electrons have been promoted to orbitals which are unoccupied in the reference.

\section{$2.2 \mathrm{X}$-ray matter interaction}

In this section the electromagnetic field and its interaction with electronic states is discussed. To introduce the notation, I first give the quantized description of the electromagnetic field. Then, the interactions of a molecule with an X-ray field will be discussed. This is done by going through the relevant approximation steps deriving the Pauli master equation and discussing the conditions under which they are valid. Finally, I will deduce transition rates for the most relevant X-ray matter interaction processes.

\subsubsection{The electromagnetic field}

Like the multi-electron problem, the electromagnetic field is a multi-particle state. However, in contrast to the electronic case, it consists of non-interacting particles with bosonic exchange symmetry. The electric field $\mathbf{E}(\mathbf{r}, t)$ and magnetic field $\mathbf{B}(\mathbf{r}, t)$ in vacuum may be 
defined by the vector potential $\mathbf{A}(\mathbf{r}, t)$ via the relations [4, 81,

$$
\mathbf{B}(\mathbf{r}, t):=\nabla \times \mathbf{A}(\mathbf{r}, t), \quad \mathbf{E}(\mathbf{r}, t):=-\alpha \frac{\partial}{\partial t} \mathbf{A}(\mathbf{r}, t),
$$

where $\alpha \simeq 1 / 137$ is the fine structure constant. In the following the Coulomb gauge is used, requiring without loss of generality

$$
\nabla \mathbf{A}(\mathbf{r}, t)=0
$$

In vacuum, the potential $\mathbf{A}$ then obeys the wave equation

$$
\Delta \mathbf{A}(\mathbf{r}, t)-\alpha^{2} \frac{\partial^{2}}{\partial t^{2}} \mathbf{A}(\mathbf{r}, t)=0
$$

The general solutions for the vector potential in a volume $V$, decomposed into the plane wave solution of Eq.2.25 in quantized form is given by the operator ${ }^{1}$

$$
\begin{aligned}
\mathbf{A}(\mathbf{r}) & =\sum_{\mathbf{k}, \lambda} \sqrt{\frac{2 \pi}{V|\mathbf{k}| \alpha}}\left(a_{\mathbf{k} \lambda} \mathbf{s}_{\lambda} e^{i \mathbf{k r}}+a_{\mathbf{k} \lambda}^{\dagger} \mathbf{s}_{\lambda} e^{-i \mathbf{k r}}\right) \\
& =: \sum_{\lambda} A_{\lambda}(\mathbf{r}) \mathbf{s}_{\lambda} \\
& =: \sum_{\mathbf{k}, \lambda} A_{\mathbf{k} \lambda}^{-}(\mathbf{r}) \mathbf{s}_{\lambda}+A_{\mathbf{k} \lambda}^{+}(\mathbf{r}) \mathbf{s}_{\lambda} .
\end{aligned}
$$

In Eq. 2.26 the sum over $\lambda$ covers the two polarization directions, described by the unit vectors $\mathbf{s}_{\lambda}$, which are perpendicular to the wave vector $\mathbf{k}$. The operators $a_{\mathbf{k} \lambda}^{\dagger}$ and $a_{\mathbf{k} \lambda}$ are bosonic creation and annihilation operators, respectively, which create a plane wave mode (photon) with wave vector $\mathbf{k}$ and polarization $\lambda$. In this representation, the Hamiltonian of the electromagnetic field simplifies to

$$
H_{\mathrm{EM}}=\sum_{\mathbf{k}, \lambda} \omega_{\mathbf{k}} a_{\mathbf{k} \lambda}^{\dagger} a_{\mathbf{k} \lambda}
$$

where $\omega_{\mathbf{k}}:=|\mathbf{k}| / \alpha$ and the zero-point energy has been shifted [34].

\footnotetext{
${ }^{1}$ Note that the vector field $\mathbf{A}(\mathbf{r})$ is in the following an operator in the Schrödinger picture and thus is not time-dependent; the time-evolution is shifted to the states of the electromagnetic field.
} 


\section{Theory and numerical implementation}

\subsubsection{Molecule-field interaction}

In the following, I discuss the relevant interaction amplitudes for a molecule exposed to an X-ray field, which will be later used to derive expressions for cross sections and decay rates.

The dynamics of a free electron in an external electromagnetic field is described using its canonical momentum

$$
\mathbf{p}+\alpha \mathbf{A}(\mathbf{r})
$$

by the Hamiltonian

$$
h=\frac{1}{2}(\mathbf{p}+\alpha \mathbf{A}(\mathbf{r}))^{2} .
$$

Thus, the total Hamiltonian of a system consisting of a molecule and the electromagnetic field is given by

$$
H=H_{\mathrm{nuc}}+H_{\mathrm{el}}+H_{\mathrm{I}}+H_{\mathrm{EM}},
$$

where $H_{\text {nucl }}$ denotes the nuclear part, $H_{\text {el }}$ the electronic part without electromagnetic field, $H_{\mathrm{EM}}$ the electromagnetic field part, and $H_{\mathrm{I}}$ the coupling of the molecular electrons to the electromagnetic field which is given by the term

$$
H_{\mathrm{I}}=\sum_{j, j^{\prime}}\left\langle\phi_{j}\left|\alpha \mathbf{A}(\mathbf{r}) \mathbf{p}+\frac{1}{2} \alpha^{2} \mathbf{A}^{2}(\mathbf{r})\right| \phi_{j^{\prime}}\right\rangle c_{j}^{\dagger} c_{j^{\prime}} .
$$

As specified in subsection 2.1.2 the operators $c_{j}^{\dagger}$ and $c_{j}$ create and annihilate an electron in spin orbital $j$, respectively. Note, that any direct interaction of the nuclei with the electromagnetic field was neglected here. The nuclei mass is too large to respond to the fast oscillating X-ray field and thus, the nuclear motion is barely affected directly by the radiation.

Inserting the quantized electromagnetic field $\mathbf{A}(\mathbf{r})$ from Eq. 2.26 into the interaction matrix element $H_{\mathrm{I}}$ (Eq. 2.31) one can see, that in first order the last term in Eq. 2.31, $\mathbf{A}^{2}(\mathbf{r})$, is responsible for scattering due to the possible pairing of annihilation and creation operators $\left(a_{\mathbf{k}}^{\dagger} a_{\mathbf{k}^{\prime}}\right)$, which preserves the number of photons. Similarly, the first term, $\alpha \mathbf{A}(\mathbf{r}) \mathbf{p}$, describes absorption and emission, because it has terms that are proportional to a single creation or annihilation operator $\left(a_{\mathbf{k}}^{\dagger}\right.$ or $\left.a_{\mathbf{k}^{\prime}}\right)$ and thus changes the number of photons by one. In the following analysis, I discuss these two terms separately. 


\section{Theory and numerical implementation}

\section{Absorption and emission amplitude}

The matrix elements of eigenstates of the electronic Hamiltonian $H_{\mathrm{el}}$ satisfy

$$
H_{\mathrm{el}}\left|\psi_{i}\right\rangle=E_{i}\left|\psi_{i}\right\rangle
$$

In terms of these electronic eigenstates, the matrix elements of the interaction Hamiltonian (Eq. 2.31), $\alpha \mathbf{A}(\mathbf{r}) \mathbf{p}$, read

$$
\begin{aligned}
\alpha\left\langle\psi_{i^{\prime}}|\mathbf{A}(\mathbf{r}) \mathbf{p}| \psi_{i}\right\rangle & \simeq \alpha \mathbf{A}\left(\mathbf{r}_{0}\right) \sum_{j j^{\prime}}\left\langle\psi_{i^{\prime}}\left|c_{j^{\prime}}^{\dagger} c_{j}\right| \psi_{i}\right\rangle\left\langle\phi_{j^{\prime}}|\mathbf{p}| \phi_{j}\right\rangle \\
& =i \alpha \omega_{i^{\prime} i} \sum_{j j^{\prime}}\left\langle\psi_{i^{\prime}}\left|c_{j^{\prime}}^{\dagger} c_{j}\right| \psi_{i}\right\rangle \sum_{\lambda} A_{\lambda}\left(\mathbf{r}_{0}\right)\left\langle\phi_{j^{\prime}}\left|r_{\lambda}\right| \phi_{j}\right\rangle,
\end{aligned}
$$

where $\omega_{i^{\prime} i}:=E_{i^{\prime}}-E_{i}$, and $\left|\phi_{j}\right\rangle$ denotes the single particle electron basis. Here, it was assumed that the wavelength of the electromagnetic wave is much larger than the extent of the molecular wave function such that

$$
e^{i \mathbf{k r}} \simeq e^{i \mathbf{k r}_{0}}
$$

with $\mathbf{r}_{\mathbf{0}}$ giving now the position of the total molecule, i.e. the center of mass of the molecule. For visible light with wavelengths of several hundred nanometers, this is usually a good approximation. For X-rays with wavelengths of $\simeq 1 a_{0}$, this is not in general applicable, as the wave functions for atoms or molecules are typically of similar size. In this case, the variation of the wave phase factor in Eq. 2.35 needs to be taken into account.

However, for the lower $Z$ elements which are addressed here, the dominant contribution for the X-ray matter interaction arises from core ionization (ionization of the $1 s$ orbitals). These orbitals are indeed much smaller than the X-ray wavelength of about $1 a_{0}$. For the interaction amplitude involving $\mathrm{K}$-shell ionization, the spatial variation of $\mathbf{A}(\mathbf{r})$ may become relevant as soon as the wavelength of light approaches the size of the K-shell $\simeq$ $1 / Z$. For carbon this would require a photon energy of more than $100 \mathrm{keV}$, which is much higher than the photon energies addressed here. In contrast, the spatial variation of $\mathbf{A}(\mathbf{r})$ is relevant also for lower photon energies, when considering the interaction amplitudes involving valence shells. As these amplitudes are much smaller than the interaction amplitude with the $\mathrm{K}$ shells and here the general evolution of the electronic state is considered, the spatial variation of $\mathbf{A}(\mathbf{r})$ can practically be neglected. Thus, in this work, the so called dipole approximation (Eq. 2.35) can safely be used to estimate the molecular ionization amplitudes. 


\section{Theory and numerical implementation}

\section{Scattering amplitude}

To discuss the remaining $\mathbf{A}^{2}$ part of the interaction Hamiltonian (Eq. 2.31), matrix elements in terms of states of the electromagnetic field and of the electronic structure are now considered. Specifically, the matrix elements

$$
\frac{\alpha^{2}}{2}\left\langle\psi_{i^{\prime}}\left|\left\langle(N-1)_{\left[\mathbf{k}_{\text {in }}, \lambda_{\text {in }}\right]} ; 1_{\left[\mathbf{k}_{\text {out }}, \lambda_{\text {out }}\right]}\left|\mathbf{A}^{2}(\mathbf{r})\right| N_{\left[\mathbf{k}_{\text {in }}, \lambda_{\text {in }}\right]}\right\rangle\right| \psi_{i}\right\rangle
$$

are examined. Here, $\left|\psi_{i}\right\rangle$ and $\left|\psi_{i^{\prime}}\right\rangle$ are again eigenstates of $H_{\mathrm{el}},\left|N_{\left[\mathbf{k}_{\text {in }}, \lambda_{\text {in }}\right]}\right\rangle$ and

$$
\left|(N-1)_{\left[\mathbf{k}_{\text {in }}, \lambda_{\text {in }}\right]} ; 1_{\left[\mathbf{k}_{\text {out }}, \lambda_{\text {out }}\right]}\right\rangle:=\frac{1}{\sqrt{N}} a_{\mathbf{k}_{\text {out }}, \lambda_{\text {out }}}^{\dagger} a_{\mathbf{k}_{\text {in }}, \lambda_{\text {in }}}\left|N_{\left[\mathbf{k}_{\text {in }}, \lambda_{\text {in }}\right]}\right\rangle
$$

is an eigenstates of $H_{\mathrm{EM}}$, where $\left|N_{\left[\mathbf{k}_{\text {in }}, \lambda_{\text {in }}\right]}\right\rangle$ has $N$ bosons in the mode $\mathbf{k}_{\mathrm{in}}, \lambda_{\mathrm{in}}$.

This choice is motivated by the fact that these matrix elements give the amplitude for scattering, as here the total number of occupied modes in the radiation field is not affected while one radiation field excitation is transferred from mode $\mathbf{k}_{\text {in }} \lambda_{\text {in }}$ to $\mathbf{k}_{\text {out }} \lambda_{\text {out }}$. The matrix elements between these states are

$$
\begin{gathered}
\frac{\alpha^{2}}{2}\left\langle\psi_{i^{\prime}}\left|\left\langle(N-1)_{\left[\mathbf{k}_{\text {in }}, \lambda_{\text {in }}\right]} ; 1_{\left[\mathbf{k}_{\text {out }}, \lambda_{\text {out }}\right]}\left|\mathbf{A}^{2}(\mathbf{r})\right| N_{\left[\mathbf{k}_{\text {in }}, \lambda_{\text {in }}\right]}\right\rangle\right| \psi_{i}\right\rangle \\
=\frac{2 \pi \delta_{\lambda_{\text {in }}, \lambda_{\text {out }}}}{V \sqrt{\omega_{\mathbf{k}_{\text {in }}} \omega_{\mathbf{k}_{\text {out }}}}} \sqrt{N}\left\langle\psi_{i^{\prime}}\left|e^{i\left(\mathbf{k}_{\text {in }}-\mathbf{k}_{\text {out }}\right) \mathbf{r}}\right| \psi_{i}\right\rangle \\
=\frac{2 \pi \delta_{\lambda_{\text {in }}, \lambda_{\text {out }}}}{V \sqrt{\omega_{\mathbf{k}_{\text {in }}} \omega_{\mathbf{k}_{\text {out }}}}} \sqrt{N} \int d \mathbf{r} e^{i\left(\mathbf{k}_{\text {in }}-\mathbf{k}_{\text {out }}\right) \mathbf{r}} \rho_{\mathrm{el}, i^{\prime} i}^{\text {trans }}(\mathbf{r}),
\end{gathered}
$$

where $\rho_{\mathrm{el}, i^{\prime} i}^{\mathrm{trans}}$ is the transition electron density for the states $\left|\psi_{i}\right\rangle$ and $\left|\psi_{i^{\prime}}\right\rangle$,

$$
\rho_{\mathrm{el}, i^{\prime} i}^{\mathrm{trans}}:=\sum_{\alpha \beta}\left\langle\psi_{i^{\prime}}\left|c_{\beta}^{\dagger} c_{\alpha}\right| \psi_{i}\right\rangle \phi_{\beta}^{*}(r) \phi_{\alpha}(r) .
$$

For $i=i^{\prime}$, the transition electron density $\rho_{\mathrm{el}, i^{\prime} i}^{\mathrm{trans}}$ is equivalent to the electron density of state $i$. In this case, the scattering is proportional to the Fourier transform of the electron density at the scattering wave vector $\mathbf{k}_{\text {in }}-\mathbf{k}_{\text {out }}$. This case represents elastic scattering, where the electronic state is not changed. In contrast, the case $i \neq i^{\prime}$ represents inelastic scattering (Compton scattering), where energy is transferred between the radiation field and the molecule. As can be seen, the scattering amplitude is proportional to the square root of the number of boson excitations (photons) $N$ in the mode $\mathbf{k}_{\text {in }}, \lambda_{\text {in }}$. Thus, the probability of the scattering processes described by the $\mathbf{A}^{2}$ term is 


\section{Theory and numerical implementation}

proportional to the number of boson excitations, i.e., to the intensity of the incident light.

In summary, I reviewed here the basic concepts of X-ray physics. To clarify the notation and be self-contained, I have discussed the interaction amplitudes for X-ray scattering, photo emission and photo absorption.

\subsubsection{Derivation of the Pauli master equation}

In the previous subsection, I discussed the relevant amplitudes for X-ray matter interaction. These amplitudes already give first insight into the elementary interaction processes. However, a full description of the dynamics of molecular states and the many more electromagnetic field states is too involved. As a simplification, I consider the dynamics of the molecule in the framework of the Pauli master equation.

In this framework, the molecule is described by time-dependent populations $n_{i}(t)$, i.e. probabilities for a molecular state $i$ to be occupied at a time $t$. The dynamics of these populations is specified via transition rates from one state to another. This way, for example, the double ionization of a molecule, consists of two independent sequential ionization processes. Although this approach seems to be a quite intuitive description of the problem, the application of the Pauli master equation is only valid under specific conditions. To show on what assumptions these conditions rely, I discuss in the following the derivation of the Pauli master equation starting from fundamental equations. This presentation closely follows Ref. [20], which is adopted here for the ionization and Auger interaction processes of a molecule. The radiation field and the electronic continuum are here treated as reservoir driving the decoherence of the electronic states of the molecule.

The derivation is split into two parts: first, the derivation of the Bloch-Redfield equation and second, the so called secular approximation. I will summarize the relevant assumptions and discuss their applicability to the problem of a molecule exposed to

XFEL radiation. Based on the derived expressions for the Redfield tensor elements, I will finally give expressions for the transition rate for the elementary interaction processes. 


\section{Theory and numerical implementation}

\section{Bloch-Redfield equation}

Similar to the discussion in subsection 2.2.1, the evolution of a system consisting of a molecule and a reservoir is described by the Hamiltonian

$$
H=H_{\mathrm{M}}+H_{\mathrm{I}}+H_{\mathrm{R}},
$$

where $H_{\mathrm{M}}$ is the Hamiltonian of the molecule (nuclear and electronic part), $H_{\mathrm{R}}$ the Hamiltonian of the reservoir, later specified as radiation field or electronic continuum, and $H_{\mathrm{I}}$ is the reservoir-molecule interaction part, which can be written as a product of operators acting on molecule or reservoir states, e.g., as a combination of respective field operators $A$ for molecular states and field operators $R$ for reservoir states,

$$
H_{\mathrm{I}}=A \cdot R,
$$

which will be specified later.

The Hilbert space of the entire system is spanned by the product states of molecule and reservoir states. The effective time evolution of the molecule is given by tracing out the reservoir states in the total density matrix $\rho(t)$. Thus, the reduced density matrices of the molecule and the reservoir are given by

$$
\begin{aligned}
\rho_{\mathrm{M}}(t) & :=\operatorname{Tr}_{\mathrm{R}} \rho(t), \\
\rho_{\mathrm{R}}(t) & :=\operatorname{Tr}_{\mathrm{M}} \rho(t) .
\end{aligned}
$$

It is assumed that the interaction $H_{\mathrm{I}}$ is weak and the reservoir is much larger than the molecule, such that the reservoir can be approximated by a steady state, $\rho_{\mathrm{R}}(t) \simeq$ $\rho_{\mathrm{R}}(0)$. Specifically, it is assumed that the density matrix $\rho_{\mathrm{R}}(0)$ is pure, i.e., it represents a statistical mixture of reservoir eigenstates. Under this assumption it follows that the expectation value of a reservoir field operator at any time is zero, i.e.

$$
\langle R(t)\rangle_{\mathrm{R}}:=\operatorname{Tr}_{\mathrm{R}} \rho_{R}(0) R(t)=0 .
$$

Within the interaction picture, the evolution of the density operator $\rho$ of the system driven by the coupling term $H_{\mathrm{I}}$ is determined by Heisenberg's equation of motion

$$
\frac{d}{d t} \rho(t)=-i\left[H_{\mathrm{I}}(t), \rho(t)\right] .
$$

The perturbative expansion of the time-evolution of the density matrix for a finite time 
step $\Delta t$ is given by the Dyson series

$$
\begin{gathered}
\Delta \rho(t+\Delta t):=\rho(t+\Delta t)-\rho(t)=-i \int_{t}^{t+\Delta t} d t^{\prime}\left[H_{\mathrm{I}}\left(t^{\prime}\right), \rho(t)\right] \\
-\int_{t}^{t+\Delta t} d t^{\prime} \int_{t}^{t^{\prime}} d t^{\prime \prime}\left[H_{\mathrm{I}}\left(t^{\prime}\right),\left[H_{\mathrm{I}}\left(t^{\prime \prime}\right), \rho(t)\right]\right]+\ldots
\end{gathered}
$$

Tracing out the reservoir states, this series yields

$$
\begin{gathered}
\Delta \rho_{\mathrm{M}}(t)=-i \int_{t}^{t+\Delta t} d t^{\prime} \operatorname{Tr}_{\mathrm{R}}\left[H_{\mathrm{I}}\left(t^{\prime}\right), \rho(t)\right] \\
-\int_{t}^{t+\Delta t} d t^{\prime} \int_{t}^{t^{\prime}} d t^{\prime \prime} \operatorname{Tr}_{\mathrm{R}}\left[H_{\mathrm{I}}\left(t^{\prime}\right),\left[H_{\mathrm{I}}\left(t^{\prime \prime}\right), \rho(t)\right]\right]+\ldots
\end{gathered}
$$

Evaluating the traces of the commutators under the time integrals, it can be shown 20 that each term in this series includes expectation values of products of field operators $R(t)$. Thus, the first term in Eq. 2.49 vanishes (Eq. 2.46). The second term in Eq. 2.49 is proportional to

$$
\left\langle R\left(t^{\prime}\right) R\left(t^{\prime \prime}\right)\right\rangle:=\operatorname{Tr}_{\mathrm{R}} \rho_{R}(0) R\left(t^{\prime}\right) R\left(t^{\prime \prime}\right)=: g\left(t^{\prime}-t^{\prime \prime}\right),
$$

which is the auto-correlation function of the reservoir field $R$. Because it was assumed that the reservoir is statistically stationary, the auto-correlation can only depend on the time difference $t^{\prime}-t^{\prime \prime}$ as anticipated by the definition of $g\left(t-t^{\prime}\right)$ [20. Note that the next non-vanishing term is proportional to

$$
\left\langle R\left(t^{\prime}\right) R\left(t^{\prime \prime}\right) R\left(t^{\prime \prime \prime}\right) R\left(t^{\prime \prime \prime \prime}\right)\right\rangle,
$$

and thus depends on the four-time-correlation of the reservoir.

In the following, I assume that only the two-time correlation function as the first nonvanishing term contributes to the dynamics and that all higher correlations are negligible and therefore the Dyson series (Eq. 2.49) can be truncated at second order in $H_{\mathrm{I}}$. A further and fundamental assumption is, that this reservoir correlation function decays rapidly with time $\tau=t^{\prime}-t^{\prime \prime}$ and vanishes for times larger than a certain correlation time $\tau_{c}$ that is significantly smaller than the finite time step $\Delta t$ in which the perturbative expansion is valid. 


\section{Theory and numerical implementation}

After having changed the integration variables $\left(t^{\prime \prime}=t^{\prime}-\tau\right)$, equation 2.49 reads

$$
\Delta \rho_{\mathrm{M}}(t+\Delta t) / \Delta t \simeq-1 /(\Delta t) \int_{t}^{t+\Delta t} d t^{\prime} \int_{0}^{\infty} d \tau \operatorname{Tr}_{\mathrm{R}}\left[H_{\mathrm{I}}\left(t^{\prime}\right),\left[H_{\mathrm{I}}\left(t^{\prime}-\tau\right), \rho(t)\right]\right]
$$

where the integration limit of $\tau$ has been extended to infinity exploiting the fact that $\langle R(0) R(\tau)\rangle$ vanishes within $\tau \ll \Delta t$. Note that in contrast to Eq. 2.49, the time evolution of the density operator in Eq. 2.52 is now Markovian (keeping in mind that the integrand vanishes for $\tau>\Delta t$ ), that means its evolution at time $t+\Delta t$ only depends on operator values evaluated at times $t^{\prime}$ within the finite time-step interval $[t, t+\Delta t]$

Expanding the commutator in Eq. 2.52 and performing the time integration over $t^{\prime}$ in the basis of molecular eigenstates yields the Bloch-Redfield equations 20,77 , 96

$$
\frac{\Delta \rho_{\mathrm{M}}(t)_{i j}}{\Delta t}=\sum_{n, m} e^{i\left(\omega_{i j}-\omega_{m n}\right) t} f\left(\left(\omega_{i j}-\omega_{m n}\right) \Delta t\right) K_{i j m n} \rho_{M}(t)_{m n}
$$

where the Redfield tensor is defined as

$$
\begin{gathered}
K_{i j m n}:=-\int_{0}^{\infty} d \tau \\
\langle R(0) R(\tau)\rangle\left(\delta_{j n} \sum_{r} A_{i r} A_{r m} e^{i \omega_{m r} \tau}-A_{i m} A_{n j} e^{i \omega_{m i} \tau}\right) \\
+\langle R(0) R(-\tau)\rangle\left(\delta_{i m} \sum_{r} A_{m r} A_{r j} e^{i \omega_{r n} \tau}-A_{i m} A_{n j} e^{i \omega_{j n} \tau}\right)
\end{gathered}
$$

and the abbreviations

$$
f(x):=e^{i x / 2} \frac{\sin (x / 2)}{x / 2} \text { and } \omega_{i j}:=\left(E_{i}-E_{j}\right),
$$

were used.

The Bloch-Redfield equations provide a full description of the molecule dynamics, without depending explicitly on the state of the reservoir. For practical applications, they are only applicable when a very limited subset of molecular states is involved in the dynamics. 


\section{Theory and numerical implementation}

\section{Secular approximation}

To gain a description of the molecule dynamics according to the Pauli master equation, the Bloch-Redfield equation derived in the previous subsection are here simplified by introducing the secular approximation.

In the secular approximation, all terms in Eq. 2.53 with $\omega_{i j}-\omega_{m n} \neq 0$ are neglected. This may be justified as $f\left(\left(\omega_{i j}-\omega_{m n}\right) \Delta t\right) \simeq 0$ for $\left(\omega_{i j}-\omega_{m n}\right) \gg 1 / \Delta t$, i.e., when the spacing between the energy levels is much larger than the inverse scale of the time evolution. Whether this assumption is valid for a molecule exposed to XFEL radiation will be discussed below.

The time evolution for the different density matrix elements in the secular approximation in the Schrödinger picture is given by

$$
\begin{aligned}
\frac{d}{d t} \rho_{\mathrm{M}}(t)_{i i} & =\sum_{n} K_{i i n n} \rho_{M}(t)_{n n} \\
\frac{d}{d t} \rho_{\mathrm{M}}(t)_{i j} & =-i \omega_{i j} \rho_{M}(t)_{i j}+K_{i j i j} \rho_{M}(t)_{i j},
\end{aligned}
$$

where now the difference quotient $\Delta \rho_{\mathrm{M}}(t+\Delta t) / \Delta t$ has been approximated by the time derivative $\frac{d \rho_{\mathrm{M}}(t)}{d t}$. Equations 2.56 and 2.57 are now uncoupled equations for the diagonal and non-diagonal elements of the density matrix. Equation 2.56 is the desired Pauli master equation, which describes the evolution of molecular state populations. These populations are given by the diagonal density matrix elements $\rho_{i i}$. The Redfield tensor elements $K_{i j m n}$ which are involved in Eq. 2.56 and 2.57 are

$$
\begin{aligned}
K_{\substack{i i n n \\
i \neq n}}= & 2\left|A_{n i}\right|^{2} \operatorname{Re} \int_{0}^{\infty} d \tau\langle R(0) R(\tau)\rangle e^{i \omega_{n i} \tau}, \\
K_{i i i i}= & -2 \sum_{r \neq i}\left|A_{i r}\right|^{2} \operatorname{Re} \int_{0}^{\infty} d \tau\langle R(0) R(\tau)\rangle e^{i \omega_{i r} \tau}, \\
K_{\substack{i j i j \\
i \neq j}}= & -\int_{0}^{\infty} d \tau\left[\langle R(0) R(\tau)\rangle\left(\sum_{r}\left|A_{i r}\right|^{2} e^{i \omega_{i r} \tau}-A_{i i} A_{j j}\right)\right. \\
& \left.+\langle R(0) R(-\tau)\rangle\left(\sum_{r}\left|A_{j r}\right|^{2} e^{-i \omega_{j r} \tau}-A_{i i} A_{j j}\right)\right] .
\end{aligned}
$$

Writing the correlation function in spectral representation, the time integral in Eq. 2.58 


\section{Theory and numerical implementation}

and Eq. 2.59 can now be carried out as

$$
\begin{gathered}
\operatorname{Re} \int_{0}^{\infty} d \tau\langle R(0) R(\tau)\rangle e^{i \omega_{n i} \tau}=\operatorname{Re} \int_{0}^{\infty} d \tau \sum_{a b} \rho_{R, a a} R_{a b} R_{b a} e^{-i \omega_{a b} \tau} e^{i \omega_{n i} \tau} \\
=2 \pi \sum_{a b} \rho_{R, a a}\left|R_{a b}\right|^{2} \delta\left(E_{a}-E_{b}+E_{n}-E_{i}\right) .
\end{gathered}
$$

Here, $\rho_{R, a a}$ is the spectral probability to find the reservoir state $a$ with energy $E_{a}$. As can be seen, the $\tau$ integration yields energy-conserving delta functions for the energy transfers between the reservoir and the molecule.

The interpretation of the Redfield tensors is now clear: Elements $K_{i i n n}$ describe transitions in which the molecule is transferred from state $n$ to state $i$, while the reservoir changes from state $a$ to state $b$ by preserving the overall energy, i.e. $E_{a}+E_{n}=E_{b}+E_{i}$. Similarly, $K_{i i i i}$ characterizes all decaying transitions going from molecular state $i$ to any other state under influence of the reservoir.

The non-diagonal elements of the molecular density matrix $\rho_{\mathrm{M}}(t)_{i j}$ represent the coherence properties between state $i$ and $j$. In the secular approximation, they couple only to themselves via the complex tensor element $R_{i j i j}$. The imaginary part of the tensor element $R_{i j i j}$ causes $\rho_{\mathrm{M}}(t)_{i j}$ to oscillate with a shifted frequency $\omega_{i j}+\operatorname{Im} K_{i j i j}$. The real part of $K_{i j i j}$ results in a decay describing the loss of coherence due to the interaction of the molecule with the bath. Having carried out the $\tau$ integration in Eq. 2.61 the rate of coherence loss is thus given by

$$
\begin{aligned}
\operatorname{Re} K_{\substack{i j i j \\
i \neq j}=} & \frac{1}{2}\left(K_{i i i i}+K_{j j j j}\right) \\
& -2 \pi \sum_{a b} \rho_{R, a a}\left|R_{a b}\right|^{2} \delta\left(E_{a}-E_{b}\right)\left(\left|A_{i i}\right|^{2}+\left|A_{j j}\right|^{2}-2 \operatorname{Re} A_{i i} A_{j j}\right) .
\end{aligned}
$$

Both terms are strictly negative (see Eq. 2.59), illustrating that in the secular approximation, the interaction with the reservoir inevitably introduces a loss of coherence of the molecular states specified by the steady oscillatory decay of the non-diagonal components of the density matrix $\rho_{\mathrm{M}}(t)_{i j}$. Given that the above assumptions hold, the evolution of the molecular density matrix can be considered completely decoherently over longer time scales. Thus, the dynamics of the molecule is solely given by the Pauli master equation (Eq. 2.56) and $\rho_{\mathrm{M}}(t)_{i j}=0$ (instead of Eq. 2.57). 


\section{Theory and numerical implementation}

\section{Validity of approximation}

In summary, the validity of the Pauli master equation is based on the following assumptions:

- The reservoir is always in a stationary state, which is a statistical mixture of eigenstates. This is particularly the case, when the reservoir is in thermodynamic equilibrium. The reservoir, which is considered here to be the X-ray field or the electronic continuum, has a large number of possible excitation modes compared to those of the molecule. The perturbation introduced by the molecule on the reservoir can be considered negligible. Therefore it is justified, to assume the reservoir field to be stationary within the timescale of $\tau_{\mathrm{dyn}}$ in which the dynamics of the molecule occurs.

- The Dyson series is truncated at the first non-vanishing order. This is valid, when the typical magnitude of the interaction strength is small compared with the typical electron binding energies in the molecule. Considering as reservoir an incident X-ray beam of frequency $\omega$, the typical interaction strength is given by the ponderomotive energy defined by

$$
E_{p}:=J /\left(4 \omega^{2}\right),
$$

where $J$ is the intensity of the electromagnetic field. The extreme intensities $J$ provided by XFEL can be treated perturbatively because the ponderomotive energy $E_{p}$ is usually still small compared to electron binding energies of a few eV. Note that the second non-vanishing term (fourth order in $H_{I}$ ) of the Dyson series would include instantaneous two-photon absorption processes [42], which have little relevance for XFEL conditions [79]. Similarly, those terms of fourth order in $H_{I}$ which describe the molecule-continuum interaction would account for instantaneous double Auger processes, i.e., the reoccupation of an electron hole via instantaneous emittance of two electrons. Considering additional mixed terms of the radiation field and the electronic continuum interaction of fourth order in $H_{I}$, these terms would give rise to a one-step approach of the Auger effect [43. By excluding these terms, the photo-ionization and the subsequent Auger decay is seen here to be composed of two distinct steps.

The consequence of truncating the Dyson series at the first non-vanishing order can also be interpreted in the following way: The interaction with the much larger reservoir behaves effectively like a measurement of the molecular quantum states. Thus, via the interaction with the reservoirs, the electronic wave function is steadily subject to measurements, such that the wave function repeatedly collapses to a 


\section{Theory and numerical implementation}

measured electronic state with the probability given by the diagonal elements of the density matrix.

- The correlation time of the radiation field $\tau_{c}$ must be smaller than the timescale $\tau_{\text {dyn }}$ with which the dynamics of density matrix elements occur. This condition is usually fulfilled, when considering the XFEL beam as reservoir, which has a coherence time of few 100 attoseconds [40], whereas the typical time scale of the dynamics is several femtoseconds. Note, that by using this strict approximation, the $\tau$ integration in Eq. 2.61 leads to energy conserving delta functions. However, in more detail, with finite correlation time and taking care of the $\tau$ dependence of the density matrix $\rho$, these energy conserving delta functions must be replaced by kernels which are broadened by the spectral width of the reservoir and the dynamics of the density matrix evolution.

- The discrete energy levels of the molecule are well separated, such that reciprocal energy differences $1 / \omega_{i j}$ are much smaller than the dynamical timescale, $1 / \omega_{i j} \ll$ $\tau_{\text {dyn }}$. When considering the different vibrational energy niveaus of the molecule, this is definitely not the case and the secular approximation becomes invalid, as the vibrational energies can lie in a molecule quite close. For the here considered XFEL-molecule interaction also dissociative molecular states are involved in which the vibrational mode levels even get continuous. However, when considering only the electronic states, the energies are usually separated by several eV corresponding to several attoseconds which is shorter than $\tau_{\text {dyn }}$.

The non-applicability of the secular approximation regarding vibrational states implies the relevance of interferences between the different vibrational levels of an electronic state (vibrational interference effects). The investigation of these vibrational levels is a formidable task, which allows deep insight in the molecular structure. However, I only focused here on the evolution of electronic state populations. I, thus, did not consider the different vibrational levels but combined them to the respective electronic state. In the electron emission spectra, the vibrational states modulate the spectra with small detailed features. By neglecting these vibrational states and their interference, I lost this information and got a more coarse-grained picture of the spectrum. In this thesis, I approximated the broadening of spectral lines due to several close-lying vibrational levels by convolution with a broadening kernel of certain width. The width of the broadening was either estimated from comparison with measured spectra or was obtained from molecular dynamics simulation as specified in detail in section 3.2 . 


\section{Theory and numerical implementation}

To perform this combination of vibrational levels to one electronic level, I assumed that the involved transition amplitudes are independent of the respective vibrational levels and thus do not depend on the nuclear coordinates $\mathbf{R}$ within the support of the vibrational states.

In the Pauli master equation two types of Redfield tensor elements $K_{i j k l}$ remain. In the following, I specify the field operators $A$ and $R$ and discuss the resulting expressions for the two Redfield tensor elements $K_{\substack{i i n n \\ i \neq n}}$ and $K_{i i i i}$.

\section{Photo-ionization and fluorescence}
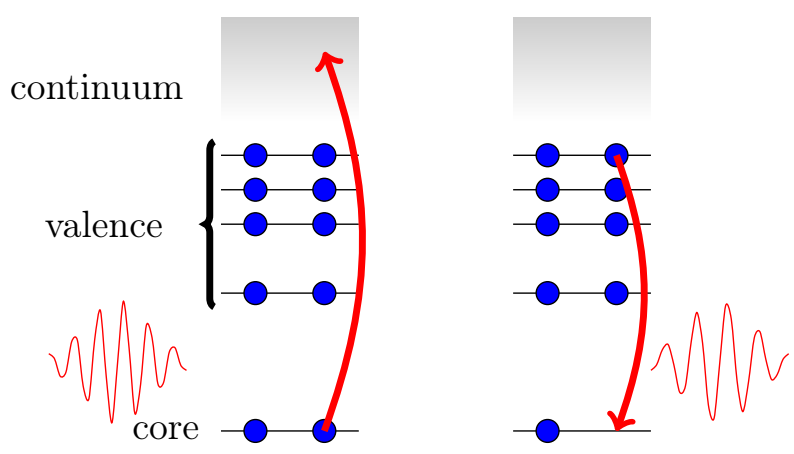

Figure 2.2: Left: Sketch of a core ionization process. The electron in the core level is transferred to an unbound level in the continuous energy spectrum. Right: Sketch of a fluorescence process. An electron from a higher energy level reoccupies the core hole while emitting a photon.

As discussed in subsection 2.2 .2 about the molecule-field interaction, I now consider the interaction Hamiltonian part relevant for photon absorption / emission from Eq. 2.31. i.e.

$$
H_{\mathrm{I}}=\alpha \mathbf{A}(\mathbf{r}) \mathbf{p}
$$

to derive ionization cross sections and fluorescence decay rates. The photo-ionization and fluorescence processes are sketched in Fig. 2.2.

The radiation field and the unbound electron states are treated here as a reservoir. To achieve this, the electron momentum operator $\mathbf{p}$ is projected onto its unbound and bound parts by introducing the projectors $Q$ and $P=1-Q$. With these projectors the interaction Hamiltonian reads

$$
H_{\mathrm{I}}=\alpha \mathbf{A}(\mathbf{r})(P \mathbf{p} P+Q \mathbf{p} P+P \mathbf{p} Q+Q \mathbf{p} Q),
$$




\section{Theory and numerical implementation}

where in the following the part $Q \mathbf{p} Q$ terms (unbound-unbound coupling) is neglected. The remaining continuum-bound and bound-bound coupling terms are

$$
\begin{aligned}
Q \mathbf{p} P & =\sum_{\alpha \kappa} \mathbf{p}_{\kappa \alpha} c_{\kappa}^{\dagger} c_{\alpha}, \\
P \mathbf{p} Q & =\sum_{\alpha \kappa} \mathbf{p}_{\alpha \kappa} c_{\alpha}^{\dagger} c_{\kappa}, \\
\text { and } \quad P \mathbf{p} P & =\sum_{\alpha \beta} \mathbf{p}_{\alpha \beta} c_{\alpha}^{\dagger} c_{\beta},
\end{aligned}
$$

where $c_{\alpha}$ and $c_{\beta}\left(c_{\alpha}^{\dagger}\right.$ and $\left.c_{\beta}^{\dagger}\right)$ are annihilation (creation) operators for bound electrons and $c_{\kappa}\left(c_{\kappa}^{\dagger}\right)$ is an annihilation (creation) operator for an unbound continuum electron. The quantities $\mathbf{p}_{\alpha \kappa}$ denote the one particle momentum matrix elements between the respective one-electron spin wave functions $\phi_{\alpha}$ and $\phi_{\kappa}$. The two Redfield tensor elements relevant in the secular approximation (Eq. 2.58 and Eq. 2.59) yield

$$
\begin{aligned}
K_{\substack{i i n n \\
i \neq n}}= & \sum_{\kappa}\left|\sum_{\beta} p_{\lambda_{q} \kappa \beta}\left\langle\psi_{i}\left|c_{\beta}\right| \psi_{n}\right\rangle\right|^{2} \frac{4 \pi^{2} \alpha}{\omega_{q}} J_{\mathbf{q} \lambda_{q}} \delta\left(E_{i}-E_{n}-\omega_{q}+\epsilon_{\kappa}\right) \\
& +\sum_{\substack{\mathbf{k}, \lambda \\
\mathbf{k} \neq \mathbf{q}, \lambda \neq \lambda_{q}}}\left|\sum_{\alpha \beta} p_{\lambda_{\alpha \beta} \beta}\left\langle\psi_{i}\left|c_{\alpha}^{\dagger} c_{\beta}\right| \psi_{n}\right\rangle\right|^{2} \frac{4 \pi^{2}}{\omega_{k} V} \delta\left(E_{i}-E_{n}+\omega_{k}\right) \\
K_{\substack{i i i i \\
i \neq n}=} & -\sum_{r \neq i} K_{r r i i} .
\end{aligned}
$$

Here, $\epsilon_{\kappa}$ is the energy of the unbound continuum electrons and it was assumed that the unbound electronic reservoir is empty at all times, i.e., when $\langle\cdot\rangle_{\text {cont }}$ means expectation value over the reservoir of the continuum electrons, $\left\langle c_{\kappa}^{\dagger} c_{\kappa}\right\rangle_{\text {cont }}=0$. Further, it was assumed that the radiation reservoir is a linearly polarized X-ray flux with photon energy $\omega_{q}$ (larger than the ionization potential of all bound electrons), polarization $\lambda_{q}$, and intensity $J_{\mathbf{q} \lambda_{q}}:=\left\langle a_{\mathbf{q} \lambda_{q}}^{\dagger} a_{\mathbf{q} \lambda_{q}}\right\rangle_{\mathrm{rad}} /(\alpha V)$, where $\langle\cdot\rangle_{\mathrm{rad}}$ means expectation value over the reservoir of the radiation field. As can be seen, two major terms in Eq. 2.69 appear, the first one is dependent on the intensity at mode $\mathbf{q}$ and describes an ionization process, the second one describes an electronic bound-bound transition, specifically a fluorescence process. 


\section{Theory and numerical implementation}

Employing the dipole approximation (Eq. 2.34) the Redfield tensor elements read

$$
\begin{gathered}
K_{\substack{i i n n \\
i \neq n}}=\sigma_{n \rightarrow i}\left(\omega_{q}\right) J_{\mathbf{q} \lambda_{q}}+\Gamma_{n \rightarrow i}^{\text {fluor. }}, \\
K_{i i i i}=-\sum_{r \neq i} \sigma_{i \rightarrow r}\left(\omega_{q}\right) J_{\mathbf{q} \lambda_{q}}-\Gamma_{i \rightarrow r}^{\text {fluor. }},
\end{gathered}
$$

with the absorption cross section

$$
\sigma_{i \rightarrow j}(\omega):=4 \pi^{2} \alpha \omega \sum_{\kappa}\left|\sum_{\beta} r_{\lambda \kappa \beta}\left\langle\psi_{j}\left|c_{\beta}\right| \psi_{i}\right\rangle\right|^{2} \delta\left(E_{i}-E_{j}-\omega+\epsilon_{\kappa}\right),
$$

and the fluorescence decay rates

$$
\begin{aligned}
\Gamma_{n \rightarrow i}^{\text {fluor. }} & :=\sum_{\lambda}\left|\sum_{\alpha \beta} r_{\lambda \alpha \beta}\left\langle\psi_{i}\left|c_{\alpha}^{\dagger} c_{\beta}\right| \psi_{n}\right\rangle\right|^{2} \sum_{\substack{\mathbf{k} \\
\mathbf{k}, \lambda \neq \mathbf{q}, \lambda q}} \frac{4 \pi^{2} \omega_{k}}{V} \delta\left(E_{i}-E_{n}+\omega_{k}\right) \\
& =\sum_{\lambda}\left|\sum_{\alpha \beta} r_{\lambda \alpha \beta}\left\langle\psi_{i}\left|c_{\alpha}^{\dagger} c_{\beta}\right| \psi_{n}\right\rangle\right|^{2} \frac{\alpha^{2}\left(E_{i}-E_{n}\right)^{3}}{2 \pi},
\end{aligned}
$$

where in the last step the summation over the radiation modes was executed in the thermodynamic limit $\left(\sum_{\mathbf{k}} \rightarrow \int d^{3} k \frac{V}{(2 \pi)^{3}}\right)$.

\section{Auger decay}

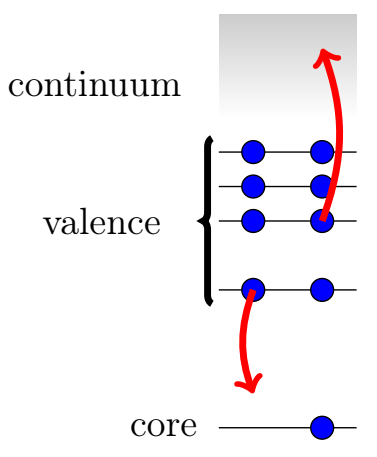

Figure 2.3: Sketch of Auger decay process. An electron at higher energy level reoccupies the core hole while another electron is emitted.

Similar to the previous consideration regarding photo-absorption and fluorescence pro- 


\section{Theory and numerical implementation}

cesses, the effect of the electronic continuum mediated by the bare continuum-bound interaction is now included in the Redfield tensors. The resulting Auger transitions are sketched in Fig. 2.3 .

Again, the total Hamiltonian is projected onto bound and continuum states

$$
H=H_{\text {bound }}+H_{\text {cont }}+H_{\mathrm{I}}=Q H Q+P H P+Q H P+P H Q .
$$

Here, $Q$ is the projector onto the Hilbert subspace with one electron in the continuum and multiple electrons in the bound states and $P$ is the projector into the multi-electron Fock space containing only the bound electrons. Note, that the two projectors $Q$ and $P$ are not exactly complementary, because multi-continuum-electron contributions have been neglected here. With the electronic Hamiltonian as specified in Eq. 2.7, the different terms are

$$
\begin{aligned}
H_{\mathrm{bound}}= & \sum_{\alpha \beta}\langle\alpha|h| \beta\rangle c_{\alpha}^{\dagger} c_{\beta}+\frac{1}{2} \sum_{\alpha \beta \gamma \delta}\left\langle\alpha \beta\left|\frac{1}{r}\right| \gamma \delta\right\rangle c_{\alpha}^{\dagger} c_{\beta}^{\dagger} c_{\delta} c_{\gamma} \\
H_{\mathrm{cont}}= & \sum_{\kappa \lambda}\langle\kappa|h| \lambda\rangle c_{\kappa}^{\dagger} c_{\lambda} \\
& +\sum_{\kappa \lambda \alpha \beta}\left(\left\langle\kappa \alpha\left|\frac{1}{r}\right| \lambda \beta\right\rangle-\left\langle\kappa \alpha\left|\frac{1}{r}\right| \beta \lambda\right\rangle\right) c_{\kappa}^{\dagger} c_{\alpha}^{\dagger} c_{\lambda} c_{\beta}, \\
H_{\mathrm{I}}= & \sum_{\alpha \kappa}\langle\alpha|h| \kappa\rangle\left(c_{\alpha}^{\dagger} c_{\kappa}+c_{\kappa}^{\dagger} c_{\alpha}\right), \\
& +\sum_{\alpha \beta \gamma \kappa}\left(\left\langle\alpha \beta\left|\frac{1}{r}\right| \kappa \gamma\right\rangle-\left\langle\alpha \beta\left|\frac{1}{r}\right| \gamma \kappa\right\rangle\right) \\
& \left(c_{\alpha}^{\dagger} c_{\beta}^{\dagger} c_{\gamma} c_{\kappa}+c_{\kappa}^{\dagger} c_{\gamma}^{\dagger} c_{\beta} c_{\alpha}\right),
\end{aligned}
$$

where the sums over $\alpha, \beta, \gamma, \delta$ are taken over the one-particle basis functions restricted to the bound states and the sums over $\kappa$ and $\lambda$ are taken over unbound continuous energynormalized states and is to be understood as an integration. With this definition and considering the electronic continuum as an empty reservoir, the two Redfield tensor elements relevant in the secular approximation (Eqs.2.58 and 2.59) yield

$$
K_{\substack{i i n n \\ i \neq n}}=\Gamma_{n \rightarrow i}^{\text {Auger }} \quad \text { and } \quad K_{i i i i}=-\sum_{r \neq i} \Gamma_{i \rightarrow r}^{\text {Auger }}
$$


with the Auger decay rate defined as

$$
\begin{gathered}
\Gamma_{i \rightarrow j}^{\text {Auger }}:=2 \pi \sum_{\kappa} \delta\left(E_{i}-E_{j}+\epsilon_{\kappa}\right) \mid \sum_{\alpha}\langle\alpha|h| \kappa\rangle\left\langle\psi_{j}\left|c_{\alpha}\right| \psi_{i}\right\rangle \\
+\left.\sum_{\alpha, \beta, \gamma}\left(\left\langle\alpha \beta\left|\frac{1}{r}\right| \kappa \gamma\right\rangle-\left\langle\alpha \beta\left|\frac{1}{r}\right| \gamma \kappa\right\rangle\right)\left\langle\psi_{j}\left|c_{\gamma}^{\dagger} c_{\beta} c_{\alpha}\right| \psi_{i}\right\rangle\right|^{2} .
\end{gathered}
$$

\section{Spin free form of the Pauli master equation}

The expressions derived in this subsection involve summations over spins. This spin summation can be carried out using the Wigner-Eckart Theorem [10]. For the relevant spin states which are singlet, doublet and triplet spin states. This yields the following relations

$$
\begin{aligned}
\left\langle\psi_{j, M=1 / 2}^{S=1 / 2}\left|c_{\alpha, \downarrow}\right| \psi_{i, M=0}^{S=0}\right\rangle & =-\left\langle\psi_{j, M=-1 / 2}^{S=1 / 2}\left|c_{\alpha, \uparrow}\right| \psi_{i, M=0}^{S=0}\right\rangle, \\
\left\langle\psi_{j, M=0}^{S=1}\left|c_{\alpha, \uparrow}\right| \psi_{i, M=1 / 2}^{S=1 / 2}\right\rangle & =\frac{1}{\sqrt{2}}\left\langle\psi_{j, M=1}^{S=1}\left|c_{\alpha, \downarrow}\right| \psi_{i, M=1 / 2}^{S=1 / 2}\right\rangle, \\
\left\langle\psi_{j, M=1 / 2}^{S=1 / 2}\left|c_{\gamma, \uparrow}^{\dagger} c_{\beta, \downarrow} c_{\alpha, \uparrow}\right| \psi_{i, M=0}^{S=0}\right\rangle & =-\left\langle\psi_{j, M=-1 / 2}^{S=1 / 2}\left|c_{\gamma, \uparrow}^{\dagger} c_{\beta, \downarrow} c_{\alpha, \downarrow}\right| \psi_{i, M=0}^{S=0}\right\rangle, \\
\left\langle\psi_{j, M=0}^{S=1}\left|c_{\gamma, \uparrow}^{\dagger} c_{\beta, \downarrow} c_{\alpha, \uparrow}\right| \psi_{i, M=1 / 2}^{S=1 / 2}\right\rangle & =\frac{1}{\sqrt{2}}\left\langle\psi_{j, M=1}^{S=1}\left|c_{\gamma, \uparrow}^{\dagger} c_{\beta, \downarrow} c_{\alpha, \downarrow}\right| \psi_{i, M=1 / 2}^{S=1 / 2}\right\rangle,
\end{aligned}
$$

where the additional $M$ and $S$ indices now denote spin quantum numbers and the arrows denote the respective spin of the fermionic creation and annihilation operators. Using the above relations, the Pauli master equation (Eq. 2.56) can be written in a spin less form where now the density matrix elements are understood as averages over the different elements of a spin multiplet.

Combining the Redfield tensors for photo-ionization, fluorescence, and Auger decay yields the spin-free Pauli master equation

$$
\begin{aligned}
\frac{d \rho_{\mathrm{M}}(t)_{i i}}{d t}= & -\sum_{r \neq i} \rho_{\mathrm{M}}(t)_{i i}\left(\Gamma_{i \rightarrow r}+\sigma_{i \rightarrow r}\left(\omega_{k}\right) J_{\mathbf{k} \lambda}\right) \\
& +\sum_{n \neq i} \rho_{\mathrm{M}}(t)_{n n}\left(\Gamma_{n \rightarrow i}+\sigma_{n \rightarrow i}\left(\omega_{k}\right) J_{\mathbf{k} \lambda}\right),
\end{aligned}
$$

where the decay rates $\Gamma_{n \rightarrow i}$ (combined Auger and fluorescence decay rates) and ionization cross sections $\sigma_{n \rightarrow i}\left(\omega_{k}\right)$ have been multiplied with the spin multiplicity of their respective final states. Accordingly, the state indices $i, r, n$ now denote respective spin multiplets. 


\section{Theory and numerical implementation}

\section{Calculation of Auger emission spectra}

In this thesis, I considered the spectra of emitted Auger electrons, particularly. Having numerically integrated the electronic spin-multiplet population $\rho_{\mathrm{M}}(t)_{i i}$ of the relevant electronic states $i$ (Eq. 2.85), Auger electron emission spectra $f(\epsilon)$ were obtained by the relation

$$
f(\epsilon)=\int_{-\infty}^{\infty} d t \rho_{\mathrm{M}}(t)_{i i} \int_{0}^{\infty} d \epsilon d\left(\epsilon-\epsilon_{n}\right) \sum_{i, n} \Gamma_{i \rightarrow n}^{\text {Auger }} .
$$

The function $d(\epsilon)$ is a broadening function, which was chosen to be a Gaussian to incorporate the effects of broadening due to nuclear motion and evolution of the density matrix (lifetime broadening) as discussed in subsection 2.2.3

In summary, under the assumptions discussed, the spin-free Pauli master euqation (Eq. 2.85) now describes the dynamics of molecular electronic spin multiplets, which were used in this work to track the evolution of a molecule exposed to XFEL radiation. The Auger electron spectra were obtained from Eq. 2.86 which accumulates the Auger emission in time weighted by the respective state population. In addition, it parametrically incorporates the effects of lifetime broadening and nuclear motion via the broadening function $d(\epsilon)$. 


\subsection{Single center method}

As discussed in the previous section, the evaluation of the dynamics of a molecule exposed to the X-ray field requires calculation of matrix elements involving electronic continuum wave functions. These electronic continuum wave functions cannot be described by localized basis functions, as commonly used in quantum chemistry. To address this problem, the wave function was expanded on a radial grid using spherical harmonics with asymptotic boundary conditions at large distances, a procedure known as single center method [26, 27]. Conceptual, this procedure roots back to the beginning of molecular ab-initio calculations [12] and has been used for various applications involving the electronic continuum in molecular calculations 64, 86, 103. In this section, I review the details of the single center method and explain how the wave function of an electron in the continuous energy spectrum in the vicinity of a molecule was numerically determined.

\subsubsection{Expansion in spherical harmonics}

As mentioned in subsection 2.2.3, it is assumed that there is only one unbound electron at a certain time. I furthermore assume that the electronic structure of the bound electrons can be determined independently of the continuum electron presence. Thus, given the many particle state of the bound electrons $|\psi\rangle$, the stationary wave function of the continuum electron $\phi_{\epsilon}(r)$ with energy $\epsilon$ is determined as solution of the stationary Schrödinger equation

$$
\begin{aligned}
-\frac{1}{2} \Delta \phi_{\epsilon}(\mathbf{r}) & +\sum_{n}^{\text {nuclei }} \frac{-Z_{n}}{\left|\mathbf{r}-\mathbf{R}_{\mathbf{n}}\right|} \phi_{\epsilon}(\mathbf{r})+\sum_{i, j}^{\text {orbitals }} \int d^{3} \mathbf{r}^{\prime} \rho_{i j} \frac{\phi_{i}^{*}\left(\mathbf{r}^{\prime}\right) \phi_{j}\left(\mathbf{r}^{\prime}\right)}{\left|\mathbf{r}-\mathbf{r}^{\prime}\right|} \phi_{\epsilon}(\mathbf{r}) \\
& -\sum_{i, j}^{\text {orbitals }} \rho_{i j}^{\sigma_{\epsilon}} \int d^{3} \mathbf{r}^{\prime} \frac{\phi_{i}^{*}\left(\mathbf{r}^{\prime}\right) \phi_{\epsilon}\left(\mathbf{r}^{\prime}\right)}{\left|\mathbf{r}-\mathbf{r}^{\prime}\right|} \phi_{j}(\mathbf{r})=\epsilon \phi_{\epsilon}(\mathbf{r}), .
\end{aligned}
$$

The first term on the left hand side of Eq. 2.87 is the kinetic energy term, the second term describes the electrostatic attraction by the molecular nuclei at positions $\mathbf{R}_{\mathbf{n}}$ with charges $Z_{n}$. The third term describes the electrostatic repulsion with the electrons occupying the molecular orbitals $\phi_{j}(\mathbf{r})$. The last term on the left side of Eq. 2.87 is the corresponding exchange interaction. The electrostatic and exchange terms depend on the matrix $\rho_{i j}$, 


\section{Theory and numerical implementation}

which is the quantum chemical density matrix, defined as

$$
\rho_{i j}:=\sum_{\sigma= \pm 1 / 2} \rho_{i j}^{\sigma}:=\sum_{\sigma= \pm 1 / 2}\left\langle\psi\left|c_{\sigma, i}^{\dagger} c_{\sigma, j}\right| \psi\right\rangle
$$

where $c_{\sigma, i}^{\dagger}$ and $c_{\sigma, i}$ create and annihilate electrons in orbital $i$ with spin $\sigma$, respectively.

The single center expansion of the molecular orbitals and the electronic continuum wave functions is given by

$$
\begin{aligned}
\phi_{\epsilon}(\mathbf{r}) & =\sum_{l m} \frac{P_{\epsilon, l m}(r)}{r} Y_{l m}(\theta, \phi), \\
\phi_{i}(\mathbf{r}) & =\sum_{l m} \frac{P_{i, l m}(r)}{r} Y_{l m}(\theta, \phi),
\end{aligned}
$$

where $Y_{l m}(\theta, \phi)$ are spherical harmonics and $P_{i, l m}(r)$ and $P_{\epsilon, l m}(r)$ are the expansion coefficients at radius $r$ of the orbital $i$ and the continuum wave function $\epsilon$, respectively. With these expansion (Eq. 2.89 and Eq. 2.90) and using the Laplace expansion for the Coulomb interaction,

$$
\frac{1}{\left|\mathbf{r}-\mathbf{r}^{\prime}\right|}=\sum_{l m} \frac{4 \pi}{2 l+1} \frac{r_{<}^{l}}{r_{>}^{l+1}} Y_{l m}^{*}\left(\theta^{\prime}, \phi^{\prime}\right) Y_{l m}(\theta, \phi),
$$

with

$$
r_{<}:=\left\{\begin{array}{ll}
r^{\prime} & \text { for } r^{\prime}<r \\
r & \text { for } r^{\prime} \geq r
\end{array} \quad r_{>}:=\left\{\begin{array}{ll}
r^{\prime} & \text { for } r^{\prime}>r \\
r & \text { for } r^{\prime} \leq r
\end{array},\right.\right.
$$

the stationary Schrödinger Eq. 2.87 for $\phi_{\epsilon}(\mathbf{r})$ yields a set of coupled differential equations for the coefficient functions $P_{\epsilon, l m}(r)$. In particular, the different parts in Eq. 2.87 
read

$$
\begin{aligned}
& -\Delta \phi_{\epsilon}(\mathbf{r})=-\frac{1}{r} \sum_{l m}\left(\frac{d^{2}}{d r^{2}} r-\frac{l(l+1)}{r^{2}}\right) P_{\epsilon, l m}(r) Y_{l m}(\theta, \phi), \\
& \frac{1}{\left|\mathbf{r}-\mathbf{R}_{\mathbf{n}}\right|} \phi_{\epsilon}(\mathbf{r})=\frac{1}{r} \sum_{\substack{l_{m}, l_{1} m_{1} \\
l_{2} m_{2}}} \frac{4 \pi\left(l_{2} m_{2}, l m \mid l_{1} m_{1}\right)}{2 l_{2}+1} \frac{r_{<}^{l_{2}}}{r_{>}^{l_{2}+1}} Y_{l_{2} m_{2}}\left(\theta_{n}, \phi_{n}\right) \\
& P_{\epsilon, l_{1} m_{1}}(r) Y_{l m}(\theta, \phi) \\
& \int d^{3} \mathbf{r}^{\prime} \frac{\phi_{j}^{*}\left(\mathbf{r}^{\prime}\right) \phi_{k}\left(\mathbf{r}^{\prime}\right)}{\left|\mathbf{r}-\mathbf{r}^{\prime}\right|} \phi_{\epsilon}(\mathbf{r})=\frac{1}{r} \sum_{\substack{l_{m}, l_{1} m_{1}, l_{2} m_{2} \\
l_{3} m_{3}, l_{4} m_{4}}} \frac{4 \pi\left(l m \mid l_{1} m_{1}, l_{4} m_{4}\right)\left(l_{4} m_{4}, l_{3} m_{3} \mid l_{2} m_{2}\right)}{2 l_{4}+1} \\
& \int_{0}^{\infty} d r^{\prime} \frac{r_{<}^{l_{4}}}{r_{>}^{l_{4}+1}} P_{j, l_{3} m_{3}}^{*}\left(r^{\prime}\right) P_{\epsilon, l_{2} m_{2}}\left(r^{\prime}\right) \\
& P_{\epsilon, l_{1} m_{1}}(r) Y_{l m}(\theta, \phi) \text {. }
\end{aligned}
$$

The abbreviation $\left(l m \mid l_{1} m_{1}, l_{2} m_{2}\right)$ denotes the Gaunt coefficients, which are integrals of spherical harmonics $Y_{l m}(\theta, \phi)$ triples,

$$
\left(l m \mid l_{1} m_{1}, l_{2} m_{2}\right)=\left(l_{1} m_{1}, l_{2} m_{2} \mid l m\right)^{*}:=\int d \Omega Y_{l m}^{*}(\theta, \phi) Y_{l_{1} m_{1}}(\theta, \phi) Y_{l_{2} m_{2}}(\theta, \phi)
$$

where the integration is to be performed over the entire $4 \pi$ solid angle,

$$
\int d \Omega:=\int_{0}^{\pi} \sin (\theta) d \theta \int_{0}^{2 \pi} d \phi
$$

With these identities (Eq. 2.93, Eq. 2.94, Eq. 2.95) the differential equation 2.87 is rewritten as

$$
\begin{gathered}
\left(\frac{-1}{2} \frac{d^{2}}{d r^{2}}+\frac{l(l+1)}{2 r^{2}}\right) P_{\epsilon, l m}(r)+\sum_{l_{1} m_{1}}\left(V_{l m, l_{1} m_{1}}^{\mathrm{ne}}(r)+J_{l m, l_{1} m_{1}}(r)\right) P_{\epsilon, l_{1} m_{1}}(r) \\
+X_{l m}\left[\mathbf{P}_{\epsilon}\right](r)=\epsilon P_{\epsilon, l m}(r)
\end{gathered}
$$

or shorter

$$
\frac{d^{2}}{d r^{2}} \mathbf{P}_{\epsilon}(r)=-\mathbf{M}(r) \mathbf{P}_{\epsilon}(r)+2 \mathbf{X}\left[\mathbf{P}_{\epsilon}\right](r)
$$

where the elements of the vector $\mathbf{P}_{\epsilon}(r)$ are the different coefficient functions $P_{\epsilon, l m}(r)$. The matrix $\mathbf{M}$ in Eq. 2.99 is defined as

$$
M_{l m, l_{1} m_{1}}(r):=\delta_{l_{1}, l} \delta_{m_{1}, m}\left(\frac{-l(l+1)}{r^{2}}+2 \epsilon\right)-2 V_{l m, l_{1} m_{1}}^{\mathrm{ne}}(r)-2 J_{l m, l_{1} m_{1}}(r)
$$


with the abbreviations

$$
\begin{aligned}
V_{l m, l_{1} m_{1}}^{\mathrm{ne}}(r):= & \sum_{n}^{\text {nuclei }} Z_{n} \sum_{l_{2} m_{2}} \frac{4 \pi\left(l_{2} m_{2}, l m \mid l_{1} m_{1}\right)}{2 l_{2}+1} \frac{r_{<}^{l_{2}}}{r_{>}^{l_{2}+1}} Y_{l_{2} m_{2}}\left(\theta_{n}, \phi_{n}\right) \\
J_{l m, l_{1} m_{1}}(r):= & \sum_{i, j}^{\text {orbitals }} \rho_{i, j} \sum_{\substack{l_{2} m_{2}, l_{3} m_{3} \\
l_{4} m_{4}}} \frac{4 \pi\left(l m \mid l_{1} m_{1}, l_{4} m_{4}\right)\left(l_{4} m_{4}, l_{3} m_{3} \mid l_{2} m_{2}\right)}{2 l_{4}+1} \\
y_{l}[f](r):= & y_{0}^{\infty} d P_{\left.i, l_{3} m_{3} P_{j, l_{2} m_{2}}\right](r)}^{\infty} \frac{r_{<}^{l}}{r_{>}^{l+1}} f\left(r^{\prime}\right) .
\end{aligned}
$$

The term $\mathbf{X}\left[\mathbf{P}_{\epsilon}\right]$ denotes the contributions of the non-local exchange interaction, which is a linear functional of the solution vector $\mathbf{P}_{\epsilon}$,

$$
\begin{gathered}
X\left[\mathbf{P}_{\epsilon}\right]_{l, m}(r):=\sum_{i, j}^{\text {orbitals }} \rho_{i j}^{\sigma_{\epsilon}} \sum_{\substack{l_{1} m_{1}, l_{2} m_{2} \\
l_{3} m_{3}, l_{4} m_{4} \\
l_{l_{4}}\left[P_{i, l_{3} m_{3}}^{*} P_{\epsilon, l_{2} m_{2}}\right](r) P_{j, l_{1} m_{1}}(r) .}} \frac{4 \pi\left(l m \mid l_{1} m_{1}, l_{4} m_{4}\right)\left(l_{4} m_{4}, l_{3} m_{3} \mid l_{2} m_{2}\right)}{2 l_{4}+1}
\end{gathered}
$$

For computational purposes, it is convenient to introduce [27 the auxiliary linear functional $y_{l}[f](r)$ (Eq. 2.103), which is implicitly determined by the boundary value problem

$$
\begin{array}{rll}
\frac{d^{2}\left(r \cdot y_{l}[f](r)\right)}{d r^{2}} & =\frac{l(l+1)}{r^{2}}\left(r \cdot y_{l}[f](r)\right)-\frac{2 l+1}{r} f(r) \\
\left(r \cdot y_{l}[f](r)\right) & \stackrel{r \rightarrow \infty}{\rightarrow} \frac{1}{r^{l}} \int_{0}^{\infty} d r^{\prime}\left(r^{\prime}\right)^{l} f\left(r^{\prime}\right) d r^{\prime} \\
\left(r \cdot y_{l}[f](r)\right) & \stackrel{r \rightarrow 0}{\rightarrow} r^{l+1} \int_{0}^{\infty} d r^{\prime} \frac{1}{\left(r^{\prime}\right)^{l+1}} f\left(r^{\prime}\right) d r^{\prime},
\end{array}
$$

resulting in a differential equation for $r \cdot y_{l}[f](r)$ which is similar to that for the quantities $P_{\epsilon, l m}(r)$.

In summary, by using the single center expansion (Eq. 2.89 and Eq. 2.90), the original three dimensional differential equation (Eq. 2.87) transforms into a set of coupled linear differential equations (Eq. 2.99). However, solving Eq. 2.99 numerically can be still quite costly depending on the number of angular quantum numbers $l, m$ which are taken account of. Therefore, I here briefly give some details on how I calculated the quantities $V_{l m, l_{1} m_{1}}(r)$ and $X_{l m}(r)$ efficiently by avoiding duplicate calculations. 


\section{Theory and numerical implementation}

To perform the single center expansion of the bound electron orbitals (Eq. 2.90), the angular integral

$$
P_{i, l m}(r)=r \cdot \int d \Omega \phi_{i}(\mathbf{r}) Y_{l m}^{*}(\theta, \phi)
$$

was calculated via Gauss-Legendre integration, where the bound electron orbitals were taken from a prior Hartree-Fock calculation performed with the PSI3 quantum package [24. Subsequently, the auxiliary quantities $y_{l}\left[P_{i, l_{1} m_{1}} P_{j, l_{2} m_{2}}\right](r)$ (see Eq. 2.103) were calculated by solving the differential equation (Eq. 2.105) for each required pair of orbital and angular quantum numbers. The numerical procedure for solving this differential equation is similar to the one described later for solving the coupled differential equation 2.98 for $P_{\epsilon, l m}(r)$. From the stored auxiliary quantities $y_{l}\left[P_{i, l_{1} m_{1}} P_{j, l_{2} m_{2}}\right](r)$, the quantities $J_{l m, l_{1} m_{1}}(r)$ and $X_{l m}(r)$ were assembled as defined in Eq. 2.102 and Eq. 2.104, respectively. The quantum chemical density matrix elements $\rho_{i j}$ were taken from the CI calculation for the respective electronic bound state. To reduce the computational costs, real valued spherical harmonics were used which are given by

$$
Y_{l m}(\theta, \phi)= \begin{cases}-i / \sqrt{2}\left(Y_{l-m}^{c}(\theta, \phi)-(-1)^{m} Y_{l m}^{c}(\theta, \phi)\right) & m<0 \\ Y_{l m}^{c}(\theta, \phi) & m=0, \\ 1 / \sqrt{2}\left(Y_{l m}^{c}(\theta, \phi)+(-1)^{m} Y_{l-m}^{c}(\theta, \phi)\right) & m>0\end{cases}
$$

where $Y_{l m}^{c}(\theta, \phi)$ are the usual complex spherical harmonics. Also, precalculated values for the Gaunt coefficients $\left(\operatorname{lm} \mid l_{1} m_{1}, l_{2} m_{2}\right)$ were used [75]. Notably, for many combinations of lm quantum number tuples the computation could be avoided, as the respective Gaunt coefficients $\left(l_{1} m_{2} \mid l_{2} m_{3}, l_{3} m_{3}\right)$ are zero [49].

\subsubsection{Asymptotics of the continuum electron wave function}

The coupled differential equations 2.98 are of second order and therefore, for each angular quantum number tuple $(l, m)$, two boundary conditions need to be specified for a unique solution. One of the boundary conditions results from the requirement, that the energy eigenvalue $\left\langle\phi_{\epsilon}|h| \phi_{\epsilon}\right\rangle$ must be finite which requires that

$$
P_{\epsilon, l m}(r \rightarrow 0) \rightarrow 0 \quad \text { for all } l, m \text {. }
$$




\section{Theory and numerical implementation}

Further boundary conditions result from the normalization condition

$$
\left\langle\phi_{\epsilon} \mid \phi_{\epsilon^{\prime}}\right\rangle=\int_{0}^{\infty} d r \sum_{l m} P_{l m, \epsilon}^{*}(r) P_{l m, \epsilon^{\prime}}(r)=\delta\left(\epsilon-\epsilon^{\prime}\right) .
$$

In this subsection, the asymptotic behavior of $\phi_{\epsilon}(r)$ is deduced by performing a perturbative expansion of the solution, from which I show, how the above normalization condition is achieved.

In the following, I will use the Dirac notation,

$$
\left\langle r, l, m \mid P_{\epsilon}^{L M}\right\rangle:=P_{\epsilon, l m}^{L M}(r),
$$

for the coefficient function at radius $r$ with the angular quantum numbers $l m$. The additional superscript $L M$ has been added to discern the different energy degenerate solutions with different contributions in the $l m$ tuples.

First, the differential equation (Eq. 2.99 for the coefficient function $P_{\epsilon, l m}^{L M}(r)$ is split into a Coulomb potential part $h_{0}$ that is caused by a single point charge and a distortion part $v$ containing the deviations of the actual molecule-continuum interaction from $h_{0}$. The value of the single point charge is chosen, such that the distortion part $v$ vanishes at a large radius $r$, i.e., the point charge is equal to the total charge of the molecule.

The solution of the boundary value problem for the single point charge potential,

$$
h_{0}\left|P_{\epsilon}^{0, L M}\right\rangle=\epsilon\left|P_{\epsilon}^{0, L M}\right\rangle
$$

with the boundary condition $\lim _{r \rightarrow 0}\left\langle r, l, m \mid P_{\epsilon}^{0, L M}\right\rangle=0$, and the normalization condition $\left\langle P_{\epsilon}^{0, L M} \mid P_{\epsilon^{\prime}}^{0, L^{\prime} M^{\prime}}\right\rangle=\delta\left(\epsilon-\epsilon^{\prime}\right) \delta_{L L^{\prime}} \delta_{M M^{\prime}}$, is known from the Coulomb wave function [1] as

$$
\left\langle r, l, m \mid P_{\epsilon^{\prime}}^{0, L M}\right\rangle=\delta_{L, l} \delta_{M, m} \mathcal{F}_{l}(r) .
$$

Here, $\mathcal{F}_{l}(r)$ denotes the regular Coulomb wave function for the respective point charge. The corresponding linearly independent solution is the irregular Coulomb wave functions $\mathcal{G}_{l}(r)$, for which $\mathcal{G}_{l}(r \rightarrow 0) \neq 0.2$ Figure 2.4 shows the regular and irregular Coulomb wave functions $\mathcal{F}_{l}(r)$ and $\mathcal{G}_{l}(r)$ for typical parameters .

\footnotetext{
${ }^{2}$ Note that in this work energy normalized Coulomb wave functions are used. Thus, $\mathcal{F}_{l}(r):=$ $\sqrt{\frac{2}{\pi k(\epsilon)}} \tilde{\mathcal{F}}_{l}(k(\epsilon) r)$, where $k(\epsilon)=\sqrt{2 \epsilon}$ and $\tilde{\mathcal{F}}_{l}(\rho)$ is the usual definition of Coulomb wave function as for example found in Ref. 1 .
} 

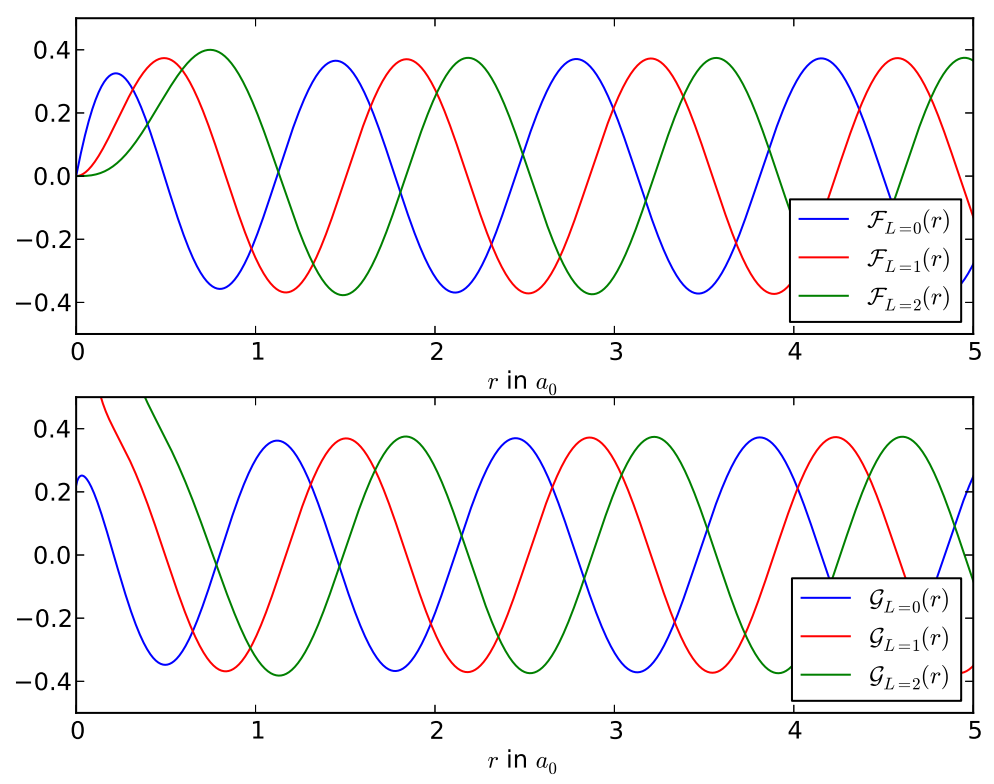

Figure 2.4: Energy normalized regular and irregular Coulomb wave function $\mathcal{F}_{L}(r)$ and $\mathcal{G}_{L}(r)$ for different angular momenta $L$ for a central point charge of $q=2$ a.u. and an energy of $\epsilon=10$ a.u. .

Next, I consider the boundary value problem for the full molecular potential (Eq.2.99)

$$
\left(h_{0}+v\right)\left|P_{\epsilon}^{L M}\right\rangle=\epsilon\left|P_{\epsilon}^{L M}\right\rangle .
$$

This boundary value problem is formally solved by the Lippmann-Schwinger equation

$$
\left|P_{\epsilon}^{L M}\right\rangle=\left|P_{\epsilon, L M}^{0}\right\rangle+G^{0}(\epsilon) v\left|P_{\epsilon}^{L M}\right\rangle
$$

where $G^{0}(\epsilon)$ is the Greens operator defined as

$$
G^{0}(\epsilon):=\lim _{\eta \searrow 0} \frac{1}{2}\left(\frac{1}{\epsilon-h_{0}+i \eta}+\frac{1}{\epsilon-h_{0}-i \eta}\right) .
$$

Iteratively reinserting the left hand side of Eq. 2.114 into the right hand side starting with $\left|P_{\epsilon}^{L M}\right\rangle=\left|P_{\epsilon, L, M}^{0}\right\rangle$, results in the Born series

$$
\left|P_{\epsilon}^{L M}\right\rangle=\left|P_{\epsilon, L, M}^{0}\right\rangle+G^{0}(\epsilon)\left(v+v G^{0}(\epsilon) v+v G^{0}(\epsilon) v G^{0}(\epsilon) v+\ldots\right)\left|P_{\epsilon, L, M}^{0}\right\rangle .
$$




\section{Theory and numerical implementation}

The spatial representation of $G^{0}(\epsilon)$ is obtained by contour integration $]^{3}$ after inserting the full set of the solution of the undistorted boundary problem (Eq. 2.111), which yields

$$
\left\langle r, l, m\left|G^{0}(\epsilon)\right| r^{\prime}, l_{1}, m_{1}\right\rangle=-\frac{\pi}{2} \mathcal{F}_{l}\left(r_{<}\right) \mathcal{G}_{l}\left(r_{>}\right) \delta_{l, l_{1}} \delta_{m, m_{1}}
$$

Inserting this expression for the Greens operator $G^{0}(\epsilon)$ into the Born series yields for $r \rightarrow \infty$

$$
\left\langle r, l, m \mid P_{\epsilon}^{L M}\right\rangle=P_{\epsilon, l, m}^{L M}(r) \stackrel{r \rightarrow \infty}{\longrightarrow} \mathcal{F}_{l}(r) \delta_{l, L} \delta_{m, M}+\mathcal{G}_{l}(r) R_{l m, L M}
$$

where the elements of the reactant matrix $\mathbf{R}$ are

$$
R_{l m, L M}:=-\frac{\pi}{2} \int_{0}^{\infty} d r^{\prime} \int_{0}^{\infty} d r^{\prime \prime} \mathcal{F}_{l}\left(r^{\prime}\right)\left\langle r^{\prime}, l, m\left|v+v G^{0}(\epsilon) v \ldots\right| r^{\prime \prime}, L, M\right\rangle \mathcal{F}_{L}\left(r^{\prime \prime}\right) .
$$

Equation 2.118 reveals that for $r \rightarrow \infty$ the elements $\left\langle r, l, m \mid P_{\epsilon}^{L M}\right\rangle$ of the solution vector $\mathbf{P}_{\epsilon}^{L M}(r)$ are given by linear combinations of the Coulomb wave function $\mathcal{F}_{l}(r)$ and $\mathcal{G}_{l}(r)$. In this asymptotic limit, the element $P_{\epsilon, L M}^{L M}(r)$ has unit contribution from the regular Coulomb function $\mathcal{F}_{L}(r)$ and contributions in the irregular Coulomb function $\mathcal{G}_{l}(r)$ specified by the matrix element $R_{L M, L M}$. Other contributions, $P_{\epsilon, l m}^{L M}(r)$ with $l, m \neq$ $L, M$, are given by the irregular Coulomb function $\mathcal{G}_{l}(r)$ with an amplitude specified by the off-diagonal elements of the reactant matrix $\mathbf{R}$. Thus, the entries in matrix $\mathbf{R}$ reflect how strong the solution differs from the solution of the undistorted boundary value problem with a Coulomb potential of a single point charge and, in particular, how strong a mixing of different angular contributions $(\mathrm{lm})$ is caused by the non-isotropic parts of the molecular potential. Notably, the reactant matrix $\mathbf{R}$ is symmetric, as $v$ and $G^{0}$ are symmetric with respect to angular components $(l m)$ (both are hermitian). This important property allows normalizing the solutions according to Eq. 2.109. It turns out that the inner products of the $\left|P_{\epsilon}^{L M}\right\rangle$ vector functions are entirely determined by their asymptotic behavior (Eq. 2.118). Therefore, it follows from the orthogonality of $\mathcal{F}_{l}(r)$ and $\mathcal{G}_{l}(r)$ that

$$
\left\langle P_{\epsilon}^{L M} \mid P_{\epsilon^{\prime}}^{L^{\prime} M^{\prime}}\right\rangle=\delta\left(\epsilon-\epsilon^{\prime}\right)\left(\delta_{L L^{\prime}} \delta_{M M^{\prime}}+\sum_{l m} R_{l m, L M} R_{l m, L^{\prime} M^{\prime}}\right)
$$

The energy normalized solutions according to Eq. 2.109 are then obtained 26] by the

\footnotetext{
${ }^{3}$ The calculation is equivalent to the calculation in Ref. 93. p. 209 for the potential free case
} 
linear combination of solution vectors

$$
\tilde{P}_{\epsilon, l m}^{L M}(r)=\frac{1}{\sqrt{1+\lambda_{L M}^{2}}} \sum_{L^{\prime} M^{\prime}} U_{L^{\prime} M^{\prime}}^{L M} P_{l m}^{L^{\prime} M^{\prime}}(r),
$$

where the vectors $\mathbf{U}^{L M}$ solve the symmetric eigenvalue problem

$$
\mathbf{R} \mathbf{U}^{L M}=\lambda_{L M} \mathbf{U}^{L M}
$$

In this way, the energy normalized (see Eq. 2.109) solutions to the perturbed radial Schrödinger equation are obtained by matching the solution vector $\mathbf{P}_{\epsilon}^{L M}$ for large $r$ with a linear combination of the regular and irregular Coulomb wave functions $\mathcal{F}_{l}(r)$ and $\mathcal{G}_{l}(r)$. The energy normalized solutions $\tilde{P}_{\epsilon}^{L M}(r)$ then serve as basis vectors for evaluation of matrix elements for Auger decay and ionization processes.

\subsection{Numerical procedure for solving the continuum wave function}

In this section, I discuss some of the numerical issues relevant for finding the solution of Eq. 2.99 .

\subsubsection{Grid points}

With an equidistant discretization of the differential equation 2.99, numerical errors may produce severe problems at those positions, where the effective potential becomes singular. This is the case at $r=0$, where for $l \neq 0$ the centrifugal term is singular and also at the positions of the nuclei, where the electrostatic attraction of the nuclei is singular. These potential singularities are handled efficiently by increasing the density of grid points at these positions, while having a lower density of grid points at those positions where the effective potential is small.

Thus, before discretizing the differential equation, I used a transformation described in Refs. [27] and [26] to generate non-equidistant radial grid points. For this purpose, I mapped the physical distance $r$ to a variable $\rho$ implicitly defined by

$$
\rho(r):=\alpha r+\beta \ln r+\sum_{p}^{\text {nuclei }} \arctan \frac{R_{p}-r}{\gamma_{p}} .
$$




\section{Theory and numerical implementation}

For positive $\alpha, \beta$, and $\gamma_{p}, \rho(r)$ is monotonously increasing and when $\rho(r)$ is discretized equidistantly by

$$
\rho_{n}=\rho_{0}+n \cdot h,
$$

the grid points $r\left(\rho_{n}\right)$ are more densely concentrated at $r \rightarrow 0$ and at the radial distances of the nuclei $R_{p}$. Further, the solutions for the transformed equidistant grid points $\rho$ were renormalized via

$$
F_{l m}(\rho):=\sqrt{\frac{d \rho(r)}{d r}} P_{l m}(r(\rho)):=\sqrt{\rho^{\prime}} P_{l m}(r),
$$

such that the differential equation 2.99 for $P_{l m}(r)$ transforms into

$$
\frac{d^{2}}{d \rho^{2}} \mathbf{F}(\rho)=-\frac{1}{\rho^{\prime 2}}(\tilde{\mathbf{M}}(\rho) \mathbf{F}(\rho)-2 \mathbf{X}[\mathbf{F}](\rho))
$$

with

$$
\tilde{M}_{l m, l_{1} m_{1}}(\rho):=M_{l m, l_{1} m_{1}}(r(\rho))+\delta_{l l_{1}} \delta_{m m_{1}}\left(\frac{1}{2} \frac{\rho^{\prime \prime \prime}}{\rho^{\prime}}-\frac{3}{4} \frac{\left(\rho^{\prime \prime}\right)^{2}}{\left(\rho^{\prime}\right)^{2}}\right)
$$

Thus, by introducing the variable transformation $\rho(r)$, the second order differential equation for $P_{l m}(r)$ yields a similar second order differential equation for the renormalized solution $F_{l m}(\rho)$, but with additional potential terms (Eq. 2.127).

\subsubsection{Vector sweep integration for Eq. 2.126 with given $\mathrm{X}$}

In this subsection, I describe how I numerically solved the coupled differential equation 2.126 for the renormalized solution $\mathbf{F}_{l m}(\rho)$. The method I used for this task is largely based on the procedure presented in Ref. [27]. Equation 2.126 is non-local because the right hand side depends on $\mathbf{X}[\mathbf{F}]$. This non-locality is treated iteratively by inserting the respective last iterate of $\mathbf{F}$ into $\mathbf{X}[\mathbf{F}]$ so that the right hand side can be considered known and one can solve the equation locally. Details of the embedding iteration are given below.

The Numerov scheme [14] is used to discretize the second order derivative in Eq. 2.126. It relates the value $\mathbf{F}_{n}$ of the function $\mathbf{F}(\rho)$ at grid point $\rho_{n}$ to its two neighboring grid points by

$$
\mathbf{A}_{n+1} \mathbf{F}_{n+1}-\mathbf{B}_{n} \mathbf{F}_{n}+\mathbf{A}_{n-1} \mathbf{F}_{n-1}=\mathbf{f}_{n}+\mathcal{O}\left(h^{4}\right) .
$$


The matrices $\mathbf{A}, \mathbf{B}$ and $\mathbf{f}$ are defined by

$$
\begin{aligned}
\mathbf{A}_{n} & :=\mathbf{1}-h^{2} \frac{1}{12} \frac{1}{\rho_{n}^{\prime 2}} \tilde{\mathbf{M}}\left(\rho_{n}\right), \\
\mathbf{B}_{n} & :=\mathbf{2}+h^{2} \frac{10}{12} \frac{1}{\rho_{n}^{\prime 2}} \tilde{\mathbf{M}}\left(\rho_{n}\right), \quad \text { and } \\
\mathbf{f}_{n} & :=h^{2} \frac{1}{12} \frac{2}{\rho_{n}^{\prime 2}}\left(\mathbf{X}\left(\rho_{n+1}\right)+10 \mathbf{X}\left(\rho_{n}\right)+\mathbf{X}\left(\rho_{n-1}\right)\right) .
\end{aligned}
$$

Together with the asymptotic boundary condition at the two outermost grid points $N$ and $N-1$,

$$
\begin{aligned}
\frac{1}{\rho_{N}^{\prime}}\left(F_{l m}^{L M}\right)_{N} & =\left(\left(\mathcal{F}_{l}\right)_{N} \delta_{L M, l m}+R_{L M . l m}\left(\mathcal{G}_{l}\right)_{N}\right), \\
\frac{1}{\rho_{N-1}^{\prime}}\left(F_{l m}^{L M}\right)_{N-1} & =\left(\left(\mathcal{F}_{l}\right)_{N-1} \delta_{L M, l m}+R_{L M . l m}\left(\mathcal{G}_{l}\right)_{N-1}\right),
\end{aligned}
$$

the boundary value problem can be written as a system of linear equations with a blocked tridiagonal matrix as

$$
\left(\begin{array}{cccccccc}
\mathbf{B}_{1} & \mathbf{A}_{2} & & & & & & \\
\mathbf{A}_{1} & \mathbf{B}_{2} & \mathbf{A}_{3} & & & & & \\
& & \ddots & & & & & \\
& & \mathbf{A}_{n-1} & \mathbf{B}_{n} & \mathbf{A}_{n+1} & & & \\
& & & & \ddots & & & \\
& & & & \mathbf{A}_{N-2} & \mathbf{B}_{N-1} & \mathbf{A}_{N} & \\
& & & & & \frac{1}{\rho_{N-1}^{\prime}} \mathbf{1} & \mathbf{0} & -\mathcal{G}_{N-1} \\
& & & & & \mathbf{0} & \frac{1}{\rho_{N}^{\prime}} \mathbf{1} & -\mathcal{G}_{N}
\end{array}\right)\left(\begin{array}{c}
\mathbf{F}_{1} \\
\mathbf{F}_{2} \\
\vdots \\
\\
\vdots \\
\mathbf{F}_{N-1} \\
\mathbf{F}_{N} \\
\mathbf{R}
\end{array}\right)=\left(\begin{array}{c}
\mathbf{f}_{1} \\
\mathbf{f}_{2} \\
\vdots \\
\\
\vdots \\
\mathbf{f}_{N} \\
\mathcal{F}_{N-1} \\
\mathcal{F}_{N}
\end{array}\right),
$$

where here, $\mathcal{F}_{n}$ and $\mathcal{G}_{n}$ are now diagonal matrices with the values of the regular and irregular Coulomb functions at grid point $n$, respectively, as diagonal elements.

This linear equation was solved by a block variant of the Thomas-Algorithm [14], which consists of two distinct steps. In the first step, the forward sweep, the lower secondary diagonal of the matrix is removed, while in the second step, the backward sweep, the upper secondary diagonal is removed. Specifically, in the backward-sweep two neighboring points of the solution vectors are related by

$$
\mathbf{F}_{n}=\mathbf{U}_{n}+\mathbf{V}_{n} \mathbf{F}_{n+1}
$$

The two matrices $\mathbf{U}_{n}$ and $\mathbf{V}_{n}$ are determined by insertion into Eq. 2.128. This gives the 


\section{Theory and numerical implementation}

iterative relation for the forward sweep

$$
\begin{aligned}
\mathbf{V}_{n} & =\left(\mathbf{B}_{n}-\mathbf{A}_{n-1} \mathbf{V}_{n-1}\right)^{-1} \mathbf{A}_{n+1} \\
\mathbf{U}_{n} & =\left(\mathbf{B}_{n}-\mathbf{A}_{n-1} \mathbf{V}_{n-1}\right)^{-1}\left(\mathbf{A}_{n-1} \mathbf{U}_{n-1}-\mathbf{f}_{n}\right) .
\end{aligned}
$$

The inner boundary conditions define the starting values for the matrix series $\mathbf{V}$ and $\mathbf{U}$ as 27

$$
\begin{aligned}
\mathbf{U}_{1} & =\mathbf{U}_{2}=0, \\
\mathbf{V}_{1}=\mathbf{V}_{2}=\mathbf{V} & =\left(\mathbf{B}_{2}-\mathbf{A}_{1} \mathbf{V}\right)^{-1} \mathbf{A}_{3},
\end{aligned}
$$

where the last equation is solved iteratively. Inserting relation 2.135 into Eq. 2.118 determines the unknown symmetric matrix $\mathbf{R}$ as

$$
\mathbf{R}=-\left(\mathbf{U}_{N-1}+\rho_{N}^{\prime} \mathcal{F}_{N} \mathbf{V}_{N-1}-\rho_{N-1}^{\prime} \mathcal{F}_{N-1}\right)\left(\rho_{N}^{\prime} \mathcal{G}_{N} \mathbf{V}_{N-1}-\rho_{N-1}^{\prime} \mathcal{G}_{N-1}\right)^{-1}
$$

where the matrix $\mathbf{U}_{N}$ is now composed of the vectors $\mathbf{U}_{N}$ from Eq.2.137.

In summary, the linear Eq. 2.126 was solved by first calculating the matrices $\mathbf{V}_{n}$ and $\mathbf{U}_{n}$ from relation 2.137. Having determined the matrix $\mathbf{R}$ via relation 2.140 , the value of the solution vector at the largest grid point $\mathbf{F}_{N}$ was determined via Eq. 2.118 and the solution vectors for all the degenerate solutions were calculated by iterating relation 2.135 backwards. The involved matrix operations were carried out using standard linear algebra libraries [3]. The evaluation of the regular and irregular Coulomb functions $\mathcal{F}_{l}(r)$ and $\mathcal{G}_{l}(r)$ was performed using the Gnu Scientific Library [38].

Note, that this block algorithm for solving Eq. 2.134 can numerically be unstable for $\left\|\mathbf{B}_{n}\right\| \leq 2\left\|\mathbf{A}_{n}\right\|$ [97] 4 The reverse condition for numerical stability is not fulfilled for all grid points. To improve the numerical stability I used a pivot strategy for the grid points where $\left\|\mathbf{B}_{n}\right\|>2|| \mathbf{A}_{n} \|$ which is similar to that used in the Gauss-elimination for nonblock tridiagonal matrices [14]. It follows, that at those grid points with $\left\|\mathbf{B}_{n}\right\|>2\left\|\mathbf{A}_{n}\right\|$, the solution has to be back iterated via

$$
\mathbf{F}_{n-1}=\mathbf{A}_{n-1}^{-1}\left(\mathbf{f}_{n}-\mathbf{A}_{n+1} \mathbf{F}_{n+1}+\mathbf{B}_{n} \mathbf{F}_{n}\right),
$$

instead of relation 2.135. The total procedure is summarized as pseudo-code in Table 2.3 ,

\footnotetext{
${ }^{4}\|\cdot\|$ is here the Frobenius matrix norm.
} 
To validate the precision of the obtained solution, the relative residual

$$
\frac{\|\mathbf{T F}-\mathbf{f}\|_{\infty}}{\|\mathbf{T}\|_{\infty}\|\mathbf{F}\|_{\infty}+\|\mathbf{f}\|_{\infty}}
$$

was verified [28], where here $\mathbf{T}$ is the block tridiagonal matrix from Eq. 2.134, $\mathbf{F}$ the matrix built up from all solution vectors column-wise, and $\mathbf{f}$ is the right hand side of Eq. 2.134 .

Table 2.3: Vector sweep algorithm: Solves the coupled boundary value problem given by Eq. 2.134 for the solution vector $\mathbf{F}_{n}$ and $\mathbf{R}$.

Given: $\mathbf{A}_{n}$ for $n=1, \ldots, N-1, \mathbf{B}_{n}, \mathbf{f}_{n}$ for $n=1, \ldots, N$

and $\mathcal{F}_{n}, \mathcal{G}_{n}, \mathcal{F}_{n}^{\prime}, \mathcal{G}^{\prime}{ }_{n}$ for $n=N, N-1$

Inner boundary condition:

$\mathbf{U}_{1}, \mathbf{U}_{2} \leftarrow \mathbf{0}$

Find $\mathbf{V}$ solving $\left(\mathbf{B}_{2}-\mathbf{A}_{1} \mathbf{V}\right)^{-1} \mathbf{A}_{3}$

$\mathbf{V}_{1}, \mathbf{V}_{2} \leftarrow \mathbf{V}$

Forward sweep:

for all $n=3, \ldots, N-1$ do

$$
\begin{aligned}
& \mathbf{V}_{n} \leftarrow\left(\mathbf{B}_{n}-\mathbf{A}_{n-1} \mathbf{V}_{n-1}\right)^{-1} \mathbf{A}_{n+1} \\
& \mathbf{U}_{n} \leftarrow\left(\mathbf{B}_{n}-\mathbf{A}_{n-1} \mathbf{V}_{n-1}\right)^{-1}\left(\mathbf{A}_{n-1} \mathbf{U}_{n-1}-\mathbf{f}_{n}\right)
\end{aligned}
$$

end for

Outer boundary condition:

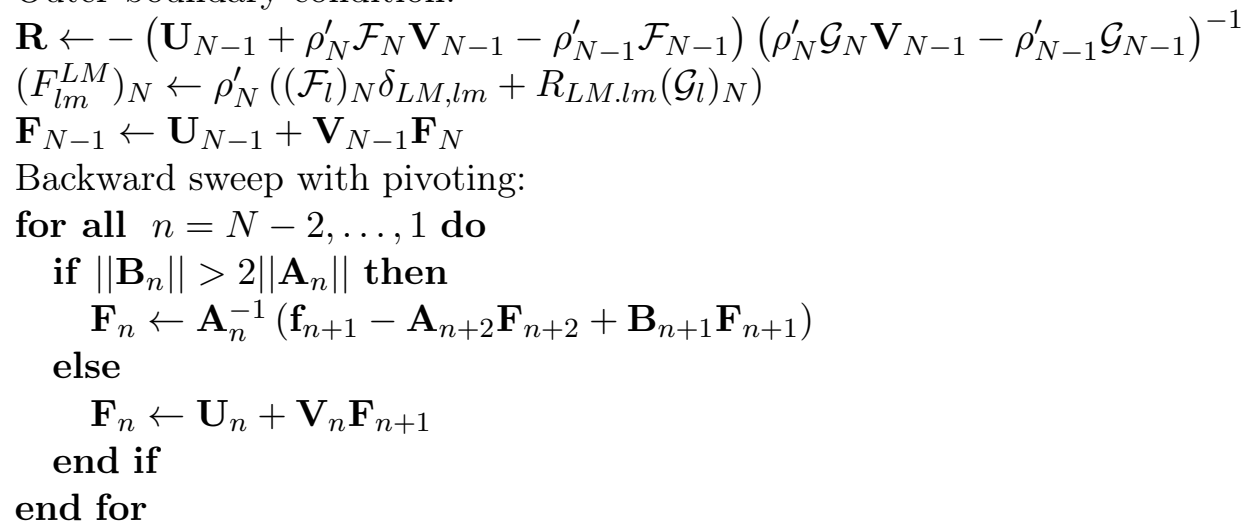

\subsubsection{Iteration of the non-local Eq. 2.126 with $\mathrm{X}[\mathrm{F}]$}

The inhomogeneity term $\mathbf{X}[\mathbf{F}]$ in the differential equation depends non-locally on the solution $\mathbf{F}$ of the differential equation. Therefore, a new solution $\mathbf{F}^{(i)}$ was determined by iteratively reinserting the previously determined solution $\mathbf{F}^{(i-1)}$ until the series defined 


\section{Theory and numerical implementation}

by

$$
\frac{d^{2}}{d \rho^{2}} \mathbf{F}^{(i)}(\rho)=-\frac{1}{\rho^{\prime 2}}\left(\tilde{\mathbf{M}}(r) \mathbf{F}^{(i)}(\rho)-2 \mathbf{X}\left[\mathbf{F}^{(i-1)}\right](\rho)\right),
$$

converges, i.e., the solution is found to be self-consistent. For the calculations performed, I confirmed the convergence by ensuring that the difference between elements of the matrix $\mathbf{R}$ in subsequent iterations is smaller than a certain threshold $\epsilon$. Specifically, I assumed the solution to be converged, if

$$
\eta_{i}:=\max _{l m, l_{1} m ;}\left|R_{l m, l_{1} m_{1}}^{i}-R_{l m, l_{1} m_{1}}^{i-1}\right|<\epsilon .
$$

As initial guess, the solution was chosen to be zero, i.e. no inhomogeneity $\mathbf{f}$ was taken into account in the first iteration. When solutions for different electronic bound states yielding slightly differing potential functions were subsequently solved, it turned out to be advantageous to use the solution for the previously determined electronic bound state as initial guess.

To accelerate the convergence of the solution, I used an acceleration scheme similar to the Aitken procedure [14]. In particular, I used the following two acceleration steps:

1. If in three subsequent iterations $i-2, i-1, i$ the quantities $\eta_{i-2}, \eta_{i-1}, \eta_{i}$ have identical signs, are drawn from the same matrix element $R_{l, m}$, and $\eta_{i}<\eta_{i-1}<\eta_{i-2}$, then the inhomogeneity for the following iteration $i+1$ is linearly extrapolated as

$$
\tilde{\mathbf{X}}=\left(1-\frac{1}{1-\eta_{i} / \eta_{i-1}}\right) \mathbf{X}\left[\mathbf{F}^{i}\right]+\frac{1}{1-\eta_{i} / \eta_{i-1}} \mathbf{X}\left[\mathbf{F}^{i-1}\right]
$$

2. If in three subsequent iterations $i-2, i-1, i$ the quantities $\eta_{i-2}, \eta_{i-1}, \eta_{i}$ have alternating signs, are drawn from the same matrix element $R_{l, m}$, and $\left|\eta_{i}\right|<\left|\eta_{i-1}\right|<$ $\left|\eta_{i-2}\right|$, then the inhomogeneity for the next iteration is linearly interpolated as

$$
\tilde{\mathbf{X}}=\left(1-\frac{1}{2} \eta_{i} / \eta_{i-1}\right) \mathbf{X}\left[\mathbf{F}^{i}\right]-\frac{1}{2} \eta_{i} / \eta_{i-1} \mathbf{X}\left[\mathbf{F}^{i-1}\right]
$$

3. Else, the inhomogeneity for the next iteration is

$$
\tilde{\mathbf{X}}=\mathbf{X}\left[\mathbf{F}^{i}\right]
$$

With these two accelerating iteration rules (1 and 2), the necessary number of iterations could be reduced significantly. 


\subsubsection{Normalization}

The procedure described in the previous subsections 2.4.2 and 2.4.3 yields solution vectors complying with the asymptotic boundary condition (see Eq. 2.118)

$$
P_{\epsilon, l, m}^{L M}(r) \stackrel{r \rightarrow \infty}{\longrightarrow} \mathcal{F}_{l}(r) \delta_{l, L} \delta_{m, M}+\mathcal{G}_{l}(r) R_{l m, L M} .
$$

Thus, the solutions are not yet properly energy normalized. As derived in subsection 2.3.2 I obtain energy normalized solutions $\tilde{P}_{\epsilon, l m}^{L M}(r)$ from the linear combination

$$
\tilde{P}_{\epsilon, l m}^{L M}(r)=\frac{1}{\sqrt{1+\lambda_{L M}^{2}}} \sum_{L^{\prime} M^{\prime}} U_{L^{\prime} M^{\prime}}^{L M} P_{l m}^{L^{\prime} M^{\prime}}(r),
$$

where the vectors $\mathbf{U}^{L M}$ are solutions to the eigenvalue problem

$$
\mathbf{R} \mathbf{U}^{L M}=\lambda_{L M} \mathbf{U}^{L M}
$$

\subsubsection{Radial truncation}

The value of the largest grid point has to be chosen such that the molecular potential has reached its asymptotic behavior within the covered radial range. At the largest grid point, the molecular potential is supposed to be similar to that of a single point charge and the solution can thus be mapped to the Coulomb wave functions $\mathcal{F}_{l}(r)$ and $\mathcal{G}_{l}(r)$. To confirm the choice of the largest grid point, I checked whether the calculated molecular potential $J_{l 1 m 1, l 2 m 2}(r)+V_{l 1 m 1, l 2 m 2}^{n e}(r)$ has relevant non-isotropic contributions, i.e. the contributions for $l 1 \neq l 2 \neq 0$ are much smaller than for $l 1=l 2=0$. For the molecules considered, a choice of $r_{\max }=15 \mathrm{a}_{0}$ turned out to be sufficient.

\subsubsection{Truncation of angular quantum numbers}

The angular expansion has to be truncated at some maximal value of angular quantum numbers $l$ and $m$ to make the calculation feasible. This relies on the assumption that higher angular momentum solutions are practically not influenced by the molecular potential as the centrifugal potential term $l(l+1) / r^{2}$ dominates for large $l$ over the molecular potential. Furthermore, depending on the angular momentum of the involved molecular orbitals in the ionization process, an electron can only be transferred into a continuum wave function with a limited angular quantum number. Thus, only the continuum solutions with limited angular momentum are accessible, and continuum wave functions 


\section{Theory and numerical implementation}

with high angular momentum do not contribute to the Auger and photo-ionization process.

The choice of the truncation of the $l, m$-angular quantum numbers is also related to the choice of center of the decomposition. A necessary but not sufficient condition for the truncation is that with the maximal angular resolution $\sim 2 \pi / l_{\max }$, all relevant molecular orbitals can be described. Usually, the core orbitals as the most localized orbitals are therefore difficult to represent within the single center expansion. For small systems containing only one central heavy atom, e.g., first row hydrides like $\mathrm{H}_{2} \mathrm{O}$, it is advantageous to set the center of decomposition on the position of the central heavy atom, e.g. oxygen in $\mathrm{H}_{2} \mathrm{O}$. With this expansion center, it turned out that the maximum $l$ quantum number in first row hydrides can be reduced to $l \leq 5$.

For molecules with two or more $\mathrm{K}$ shells, the required angular resolution is much higher. Choosing the center of decomposition at the molecule's center of mass, the absolute value of the $m$ quantum number can be reduced to some tractable maximal value. To make the calculations for linear molecules feasible, an adapted truncation scheme was used. Here, it must be considered that in a molecular photo-ionization process an emitted electron can at maximum obtain additional angular momentum of $l \pm 1$ or $m \pm 1$. For the Auger process, an emitted electron may at maximum carry away the total angular momentum of two bound electrons. Thus, e.g., the Auger decay process in acetylene (or ethyne) involving $\pi$-orbitals $(m= \pm 1$ ) can be described with $m$ quantum numbers $|m| \leq 2$, whereas the $l$ quantum numbers needs to cover much higher values, i.e. $l \leq 20$.

\subsubsection{Exchange potential approximation}

The numerical procedure presented in subsection 2.4 .3 can be simplified by avoiding the inhomogeneity term $\mathbf{X}[\mathbf{P}]$ on the right side of Eq. 2.99. Instead of the inhomogeneity term, I approximated in most calculations the exchange interaction with an exchange interaction potential. Here the Kohn Sham Gaspar (KSG) approximation 39 was used, which replaces the inhomogeneous part $\mathbf{X}[\mathbf{P}]$ by the potential term

$$
V_{\mathrm{XC}}[\rho(\mathbf{r})]=-\left(\frac{3}{\pi} \rho(\mathbf{r})\right)^{1 / 3}
$$

The cubic root of the electron density $\rho^{1 / 3}$ was numerically calculated by computing the electron density $\rho(\mathbf{r})$ at each point of a sphere from its spherical harmonic decomposi- 
tion

$$
\begin{aligned}
\rho(\mathbf{r}) & =\sum_{l m} Y_{l m}(\theta, \phi) \sum_{l_{1} m_{1}, l_{2} m_{2}}\left(l_{1} m_{1} \mid l_{2} m_{2} l m\right)^{*} \sum_{i, j} \frac{P_{i, l m}(r) P_{j, l m}^{*}(r)}{r^{2}} \rho_{i, j} \\
& =: \sum_{l m} Y_{l m}(\theta, \phi) \frac{\rho_{l m}(r)}{r}
\end{aligned}
$$

and numerically decomposing the cubic root of the electron density $\rho^{1 / 3}(\mathbf{r})$ again into the spherical harmonics by a Gauss-Legendre integration

$$
\left(\rho^{1 / 3}\right)_{l m}(r)=r \int d \Omega Y_{l m}^{*}(\theta, \phi) \rho^{1 / 3}(\mathbf{r})
$$

For small molecules, such as the first row hydrides, the electron density is mostly spherically symmetric. For these molecules I avoided the expensive numerical computation of the electron density and subsequent decomposition of its cubic root by approximating the cubic root of the electron density by the Taylor series

$$
\begin{aligned}
\rho(\mathbf{r})^{1 / 3} & =Y_{00}(\theta, \phi)^{1 / 3}\left(\rho_{00} / r\right)^{1 / 3} \\
& +1 / 3 \sum_{\substack{l \neq 0 \\
m \neq 0}} \frac{Y_{l m}(\theta, \phi) \rho_{l m}(r)}{Y_{00}(\theta, \phi) \rho_{00}(r)}+\mathcal{O}\left(\rho_{l m}(r) / \rho_{00}(r)\right)^{2},
\end{aligned}
$$

truncated at first order of the non-spherically symmetric term of $\rho_{l m}(r)$.

\subsubsection{Calculation of transition amplitudes}

Having obtained the numerical representation of the energy normalized continuum wave function, the relevant transition amplitudes involving these continuum wave function can now be calculated. The dipole matrix element, the one-particle Hamiltonian and the 


\section{Theory and numerical implementation}

Coulomb matrix elements read in the single center expansion

$$
\begin{aligned}
\left\langle\phi_{i}\left|r_{\lambda}\right| \phi_{\epsilon}^{L M}\right\rangle= & \sqrt{\frac{4 \pi}{3}} \int_{0}^{\infty} d r r \sum_{\substack{l_{1} m_{1} \\
l_{2} m_{2}}} P_{\epsilon, l_{1} m_{1}}(r) P_{i, l_{2} m_{2}}(r)\left(l_{2} m_{2} \mid l_{1} m_{1}, 1 \lambda\right), \\
\left\langle\phi_{i}|h| \phi_{\epsilon}^{L M}\right\rangle= & \sum_{l m} \int_{0}^{\infty} d r\left(\frac{1}{2} \frac{d P_{i, l m}^{*}(r)}{d r} \frac{d P_{\epsilon, l m}^{L M}(r)}{d r}\right. \\
& \left.+\sum_{\substack{l_{1} m_{1} \\
l_{2} m_{2}}} P_{i, l m}^{*}(r) V_{l m, l_{1} m_{1}}^{\mathrm{ne}}(r) P_{\epsilon, l_{2} m_{2}}^{L M}(r)\right) \\
\left\langle i j\left|\frac{1}{r}\right| k \epsilon\right\rangle= & \sum_{\substack{l m_{1}, l_{1} m_{1}, l_{2} m_{2} \\
l_{3} m_{3}, l_{4} m_{4}}}^{\infty} \frac{4 \pi\left(l_{3} m_{3} \mid l_{4} m_{4}, l m\right)\left(l m, l_{1} m_{1} \mid l_{2} m_{2}\right)}{2 l_{4}+1} \\
& \int_{0}^{\infty} d r P_{i, l_{3} m_{3}}^{*}(r) P_{k, l_{4} m_{4}}(r) y_{l}\left[P_{j, l_{1} m_{1}}^{*} P_{\epsilon, l_{2} m_{2}}^{L M}\right](r)
\end{aligned}
$$

where the indices $i, j, k$ denote bound molecular orbitals and $\epsilon$ denotes the continuum wave function, respectively. As can be seen, all of these expressions involve a single radial integration. The numerical evaluation of these matrix elements was performed on the radial grid using Simpson's rule [14]. For the calculation of the one-particle Hamiltonian matrix elements (Eq. 2.157), the derivatives $d P_{i, l m}(r) / d r$ were approximated by finite differences.

In summary, I have given in this section all relevant numerical details which were used to obtain the continuum wave function in the vicinity of a molecule and to calculate bound-continuum interaction amplitudes. Specifically, I have considered photoionization and Auger decay transitions. The evaluation of these quantities allows to determine the electron dynamics and, in particular, to calculate ionization and Auger decay rates. 


\section{Results}

\subsection{Auger decay in neon}

In this section, I validate the computational approach introduced in the previous chapter and test the convergence with respect to different basis sets, to the CI truncation, and to the number of radial grid points. I consider the Auger decay of single K-shell ionized neon, which has been well studied before [9, 19, 100].

First, I will show how the calculated energies for a core ionized initial state and, exemplarily, two different doubly valence ionized final states converge with respect to the used basis set and CI truncation scheme. Then, I will discuss the convergence of the calculated decay rates with respect to the size of the basis set, the CI truncation scheme, and the number of radial grid points used. Finally, I will compare the calculated Auger decay rates with the experimental spectrum.

\subsubsection{Convergence of initial and final state energies with respect to basis sets and $\mathrm{Cl}$ truncation}

To test the convergence with respect to the basis sets, I used four different basis sets consisting of Gaussian functions contracted to a finite number of basis functions. The basis sets and the number of contractions are listed in Table 3.1 .

In all calculations, molecular orbitals were obtained in a restricted open shell HartreeFock (ROHF) calculation for the initial, core ionized state using the PSI3 quantum chemistry package 24]. These molecular orbitals were then used in a single reference CI calculation to describe the core ionized states. In particular, the CI truncation scheme introduced in subsection 2.1 .3 was used with the additional restriction, that the core orbital is singly occupied in all involved configurations. Figure 3.1 shows the energy of the single core ionized state of neon $\left(1 s^{-1}\right)$ obtained with different basis sets and different CI truncations, respectively. With the CISD truncation scheme in combination 


\section{Results}

Table 3.1: Basis sets which were employed in the calculations and their number of contractions for neon. The letters s, p, d, f, g denote the angular symmetry of respective basis functions.

\begin{tabular}{|c|c|c|}
\hline Basis set & $\begin{array}{c}\text { Number of } \\
\text { Gaussian primitives }\end{array}$ & $\begin{array}{l}\text { Number of } \\
\text { contractions }\end{array}$ \\
\hline $3-21 \mathrm{G} 11$ & $\begin{array}{l}6 \mathrm{~s} \\
3 \mathrm{p}\end{array}$ & $\begin{array}{l}3 \mathrm{~s} \\
2 \mathrm{p}\end{array}$ \\
\hline cc-pVDZ 95 & $\begin{array}{l}10 \mathrm{~s} \\
5 \mathrm{p} \\
2 \mathrm{~d}\end{array}$ & $\begin{array}{l}4 \mathrm{~s} \\
3 \mathrm{p} \\
2 \mathrm{~d}\end{array}$ \\
\hline cc-pVTZ 95 & $\begin{array}{l}12 \mathrm{~s} \\
7 \mathrm{p} \\
3 \mathrm{~d} \\
1 \mathrm{f}\end{array}$ & $\begin{array}{l}6 \mathrm{~s} \\
5 \mathrm{p} \\
3 \mathrm{~d} \\
1 \mathrm{f}\end{array}$ \\
\hline cc-pVQZ 95 & $\begin{array}{c}15 \mathrm{~s} \\
9 \mathrm{p} \\
5 \mathrm{~d} \\
3 \mathrm{f} \\
1 \mathrm{~g}\end{array}$ & $\begin{array}{l}\mathrm{s} \\
7 \mathrm{p} \\
5 \mathrm{~d} \\
3 \mathrm{f} \\
1 \mathrm{~g}\end{array}$ \\
\hline
\end{tabular}

with the cc-pVTZ basis set, the absolute energy converged within few $\mathrm{eV} \simeq 0.1$ a.u.

The same set of molecular orbitals was used to describe final states of the Auger process. Note, that the molecular orbitals have been optimized for the core ionized states, such that the description of initial and final electronic states may be unbalanced. This imbalance is compensated by using multiple references in the configuration interaction calculations for the final double valence vacancy states. The set of references was chosen from all ten possible combinations with two holes in the four valence electron orbitals. From these references, the space of configuration state functions was built up from a finite number of excitations into the unoccupied MOs.

For two final electronic states of the K-LL Auger process, the $2 s^{-2}\left({ }^{1} S\right)$ and $2 p^{-2}\left({ }^{1} D\right)$ states, Figure 3.2 shows the calculated energies as a function CI truncation scheme for different basis sets. As can be seen, with the MRCISD truncation and the cc-pVTZ basis set, the energy converged to within $\simeq 0.1$ a.u.

Thus, for both, initial and final states, the combination of the cc-pVTZ basis set with the (MR)CISD truncation scheme provides electronic states for which the absolute energy is converged within $0.1 \mathrm{a} . \mathrm{u} . \simeq 2.7 \mathrm{eV}$. This combination is thus chosen for further 


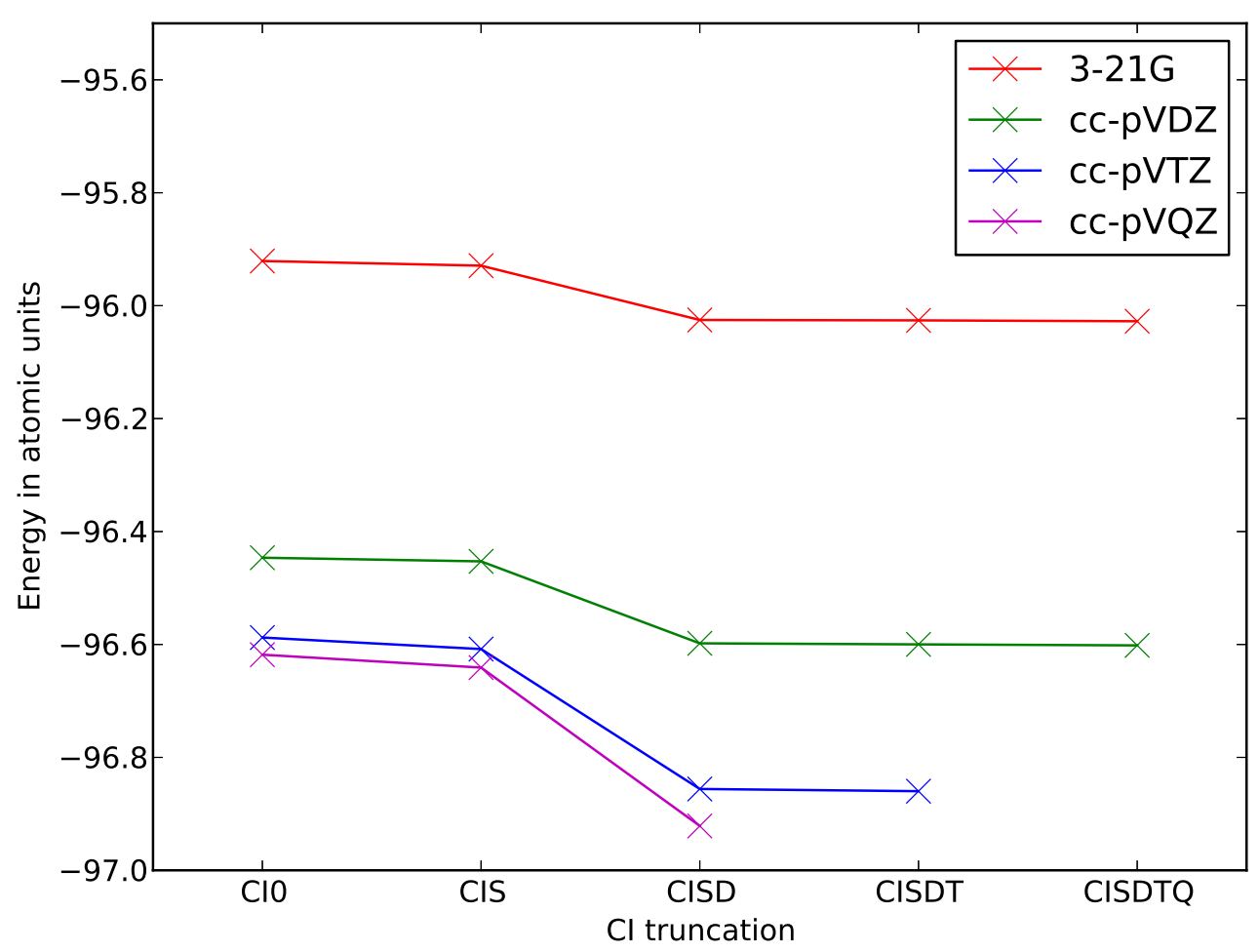

Figure 3.1: Convergence of calculated single core ionized state energy of neon with respect to CI truncation and basis set. With the CISD truncation and cc-pVTZ basis set the computed energy is converged within $\simeq 0.1$ a.u..

calculations. Note, that the energy differences, which are relevant for the calculation of Auger spectra, can be expected to be even more accurate, because most correlation effects contribute similarly to initial and final state energy and thus tend to cancel out. This is demonstrated in Fig. 3.3 where the transition energy (energy difference) for the same states, as considered in Fig. 3.2 and Fig. 3.1. is shown as a function of the CI truncation scheme and with different basis sets. As can be seen, the transition energies change by less than $1 \mathrm{eV}$ with a basis set larger than the cc-pVTZ basis set and with a CI truncation scheme beyond (MR)CISD.

Note, that relativistic contributions to the transition energies, which are of the order of $1 \mathrm{eV}[72]$, were neglected here. It can be assumed that these contributions are approximately the same for each final state, such that they may give rise to a constant shift of transition energies. 


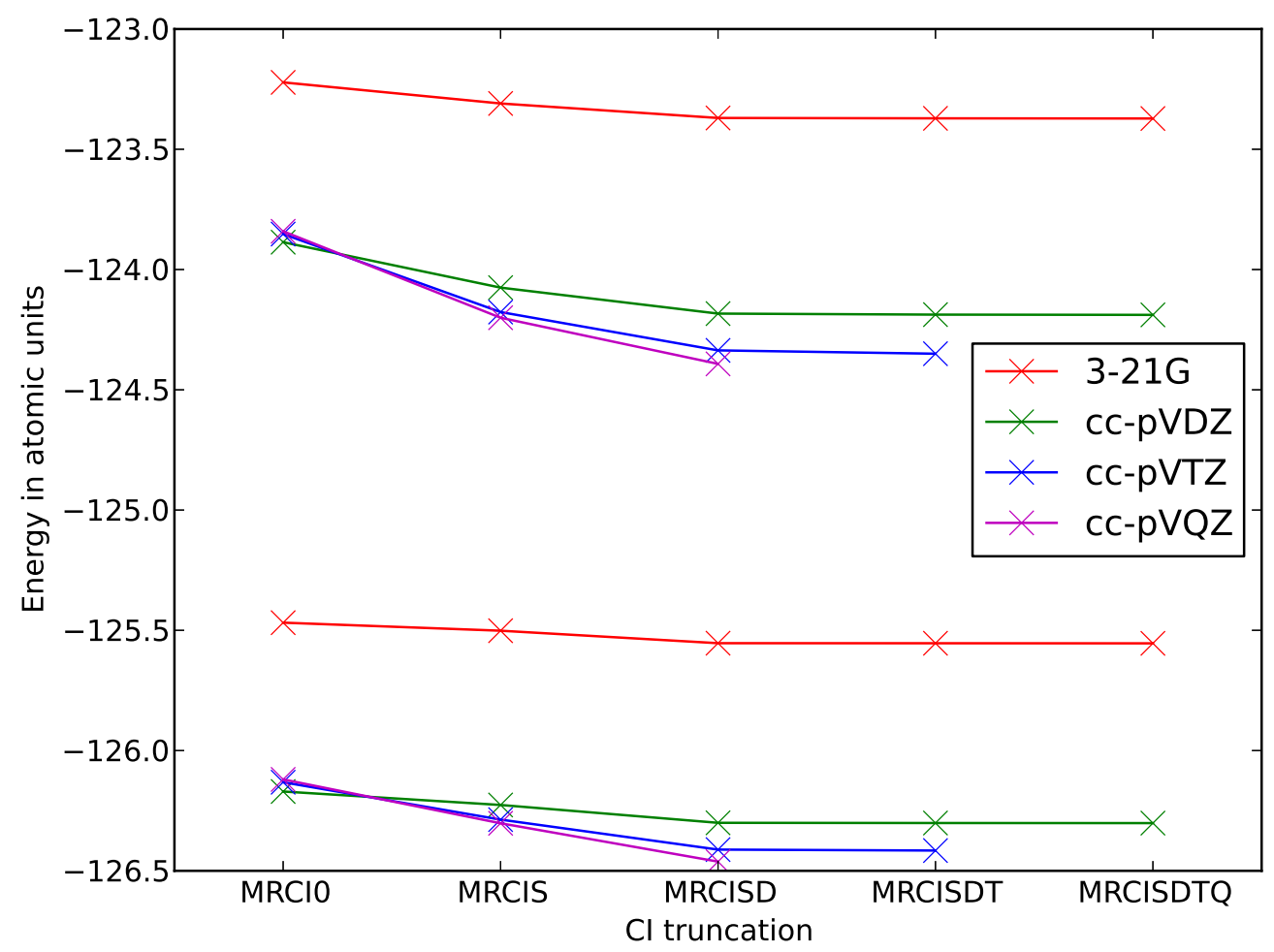

Figure 3.2: Convergence of calculated double valence ionized state energy of neon with respect to CI truncation scheme and chosen basis set. Exemplary, two states are shown: the upper crosses show the $2 s^{-2}\left({ }^{1} S\right)$ state, the lower crosses set the $2 p^{-2}\left({ }^{1} D\right)$ state. With the MRCISD truncation and the cc-pVTZ basis set, the computed energy is converged within $\simeq 0.1 \mathrm{a} . \mathrm{u}$.

In summary, for the calculations of the transition energies of neon, the combination of the cc-pVTZ basis set and the (MR)CISD truncation scheme results in transition energies which are accurate within $1 \mathrm{eV}$. This accuracy is sufficient to resolve individual features of the Auger electron emission spectrum, as the individual transition energies spread over a spectral range of about $60 \mathrm{eV}\left(\simeq\right.$ difference between $2 s^{-2}\left({ }^{1} S\right)$ and $2 p^{-2}\left({ }^{1} D\right)$ final state). 


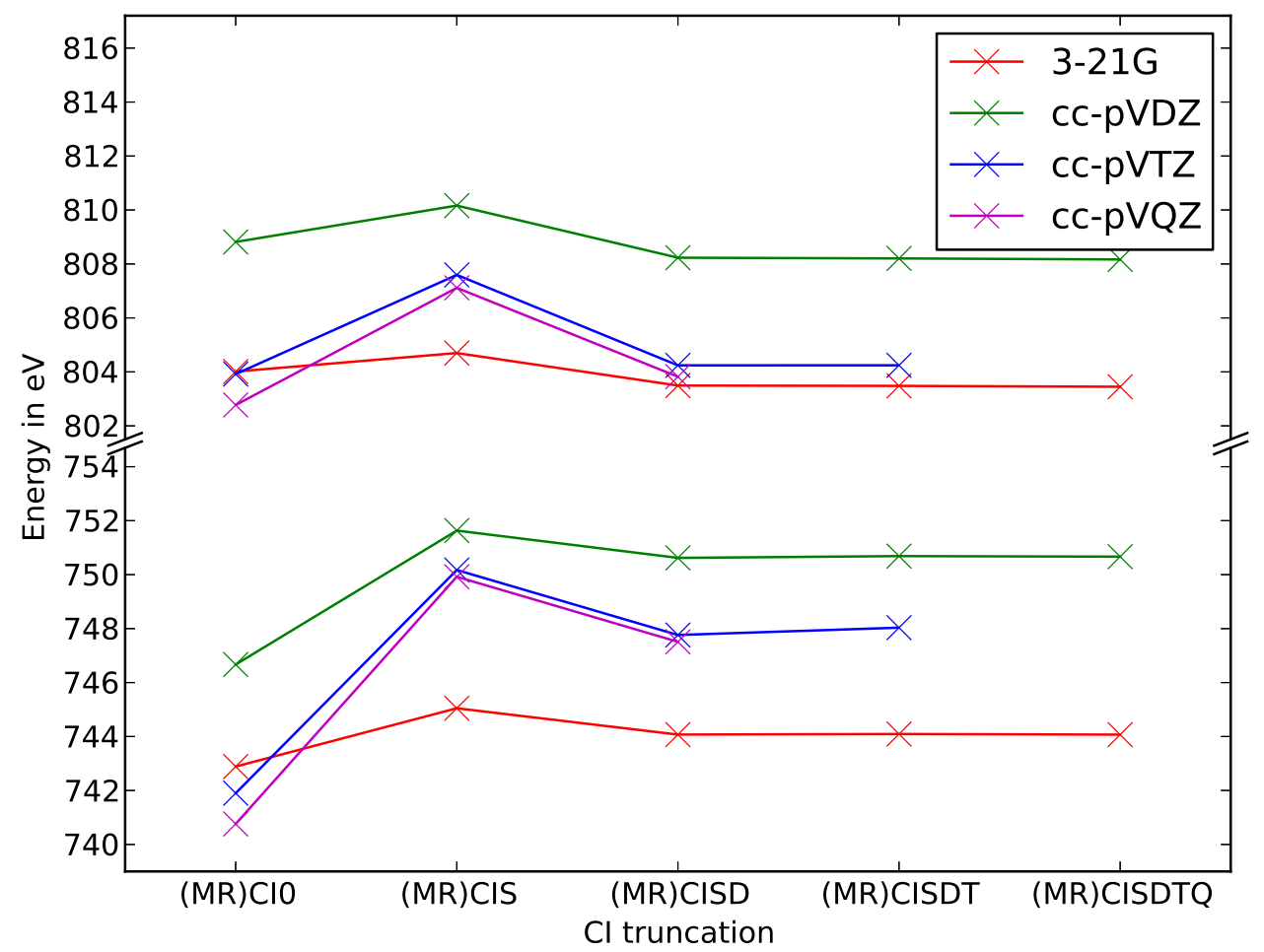

Figure 3.3: Transition energies for the K-LL Auger decay calculated with different basis sets and CI truncations. Exemplary, two states are shown: the upper crosses depict the transition energy for the $2 s^{-2}\left({ }^{1} S\right)$ final state, the lower crosses the $2 p^{-2}\left({ }^{1} D\right)$ final state. As can be seen, the transition energies at CISD level with cc-pVTZ basis change by less than $1 \mathrm{eV}$ with increased basis set and CI truncation.

\subsubsection{Convergence of transition rates with respect to basis set and $\mathrm{Cl}$ truncation}

In this subsection, the convergence of the calculated Auger transition rates, with respect to the basis set and CI truncation scheme for the K-LL Auger transition of neon, is studied. Throughout this section, 2000 radial grid points were used. The largest radial grid point was chosen to be $20 a_{0}$. The center of expansion was chosen to be at the position of the nucleus. Because of the isotropic symmetry of the problem and the number of electrons (single atom, $s$ and $p$ electrons), the angular truncation of $l m$ angular quantum 


\section{Results}

numbers to $l, m \leq 2$ is exact.

For the same two final states $\left(2 s^{-2}\left({ }^{1} S\right)\right.$ and $\left.2 p^{-2}\left({ }^{1} D\right)\right)$, which were considered in the previous section, Fig. 3.4 shows the calculated transition rates as a function of the CI truncation scheme calculated with different basis sets. For comparison, also results using exact exchange and the KSG exchange potential for the determination of the continuum electron wave function are shown. As can be seen, with increasing basis set size and CI truncation scheme the transition rates converge. Beyond the CISD truncation scheme, the calculated rates show almost no dependence on the CI truncation, whereas the dependence on the basis set is much stronger. A relative difference of less than 5\%, when changing from a cc-pVTZ to a cc-pVQZ basis set, is seen. Using the KSG exchange interaction approximation yields transition rates, which deviate slightly from the rates calculated with the exact exchange.

To further investigate the validity of the KSG exchange approximation, Table 3.2 compares all K-LL Auger transition rates for neon calculated with exact exchange to those calculated with the approximated exchange potential. The transition rates differ in all but one case by less than $5 \%$. The rate for the triplet transition $1 s^{-1} \rightarrow 2 s^{-1} 2 p^{-1}\left({ }^{3} P\right)$ differs by $14.5 \%$. The total decay rate deviates by about $3.3 \%$. Relativistic effects are known from atomic calculations 19 to be relevant for atomic numbers $Z \geq 35$ and were therefore neglected here.

Table 3.2: Calculated K-LL Auger transition rates in $10^{-3}$ a.u and transition energies in $\mathrm{eV}$ for neon (cc-pVTZ basis, CISD/MRCISD).

\begin{tabular}{l|c|c|c|c} 
Final state & $\begin{array}{c}\text { Transition } \\
\text { energy }\end{array}$ & $\begin{array}{c}\Gamma_{i \rightarrow f} \\
\text { (exact exchange) }\end{array}$ & $\begin{array}{c}\Gamma_{i \rightarrow f} \\
\text { (approx. exchange) }\end{array}$ & $\begin{array}{c}\text { Relative } \\
\text { difference }\end{array}$ \\
\hline $2 p^{-2}\left({ }^{1} D\right)$ & 804.24 & 4.903 & 5.122 & $+4.4 \%$ \\
$2 p^{-2}\left({ }^{1} S\right)$ & 800.53 & 0.716 & 0.749 & $+4.6 \%$ \\
$2 s^{-1} 2 p^{-1}\left({ }^{1} P\right)$ & 771.33 & 1.726 & 1.680 & $-2.6 \%$ \\
$2 s^{-1} 2 p^{-1}\left({ }^{3} P\right)$ & 782.14 & 0.719 & 0.823 & $+14.5 \%$ \\
$2 s^{-2}\left({ }^{1} S\right)$ & 747.76 & 0.706 & 0.688 & $-2.5 \%$ \\
\hline total rate & & 8.769 & 9.062 & $+3.3 \%$
\end{tabular}

In summary, with a (MR)CISD truncation scheme and the cc-pVTZ basis set, the transition energies are converged to within $0.1 \mathrm{eV}$, and the transition rates by about $5 \%$. The use of the KSG exchange potential approximation is justified within these error bounds. 


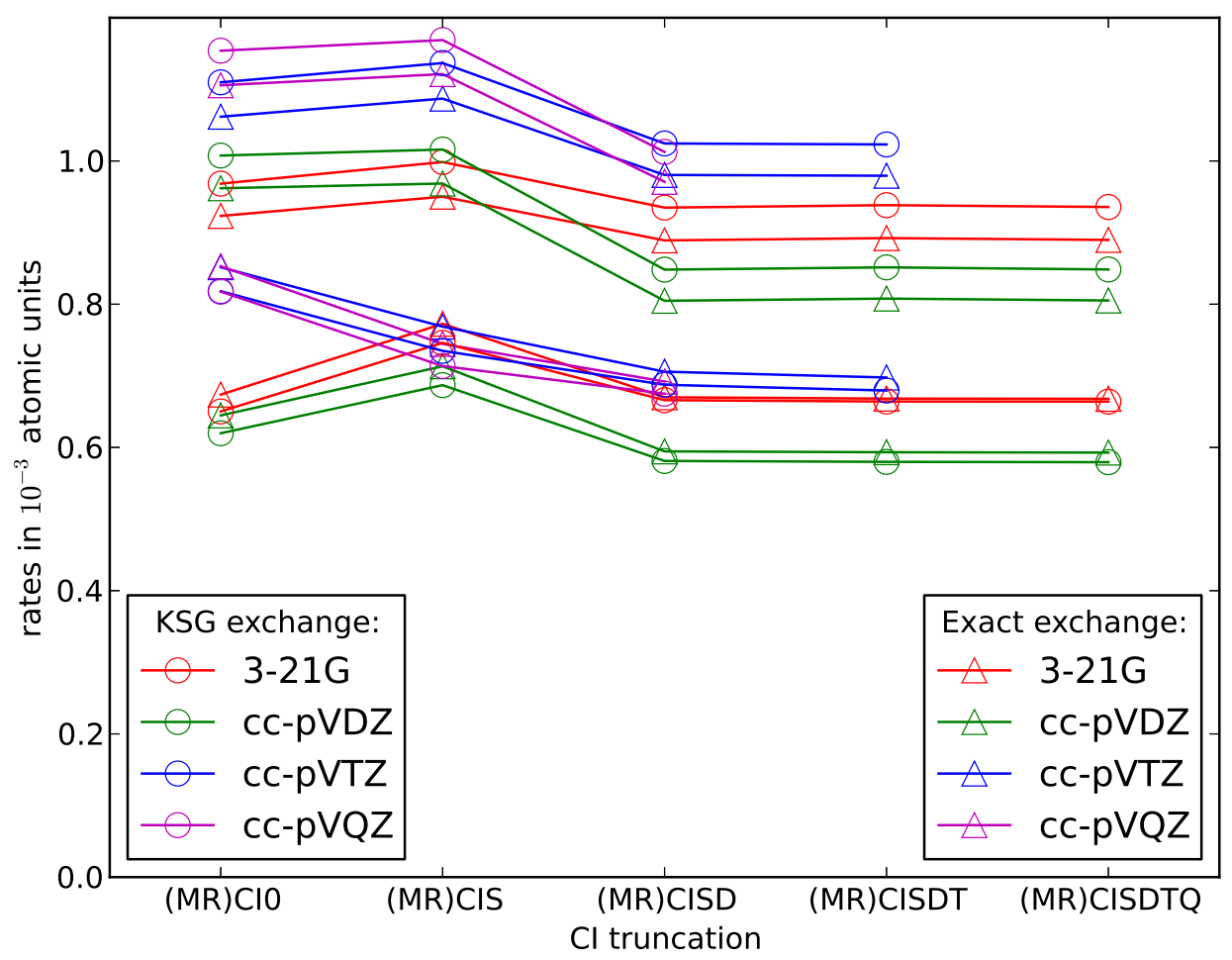

Figure 3.4: Calculated K-LL Auger transition rates with different CI truncations, basis sets, and exchange interaction models for the two final states shown in Fig. 3.2 (the upper triangles / circles show the $2 p^{-2}\left({ }^{1} D\right)$ final state, the lower triangles / circles the $2 s^{-2}\left({ }^{1} S\right)$ final state). Triangles denote rates calculated with exact exchange contribution, circles denote rates calculated with the KSG exchange approximation. The color indicates the used basis set.

\section{Convergence of decay rates with respect to the radial grid}

To validate the numerical calculation of the continuum wave function and the Auger decay rates, the calculated transition rates for the K-LL Auger decay of neon were compared using different radial integration steps and the convergence was tested. From the Numerov integration scheme, a discretization error proportional to the power of $\Delta r^{4}$ is expected. Because non-equidistant grid-spacing is used, convergence of the calculated transition rates is here considered as a function of the asymptotic grid-spacing $\Delta r$ at 


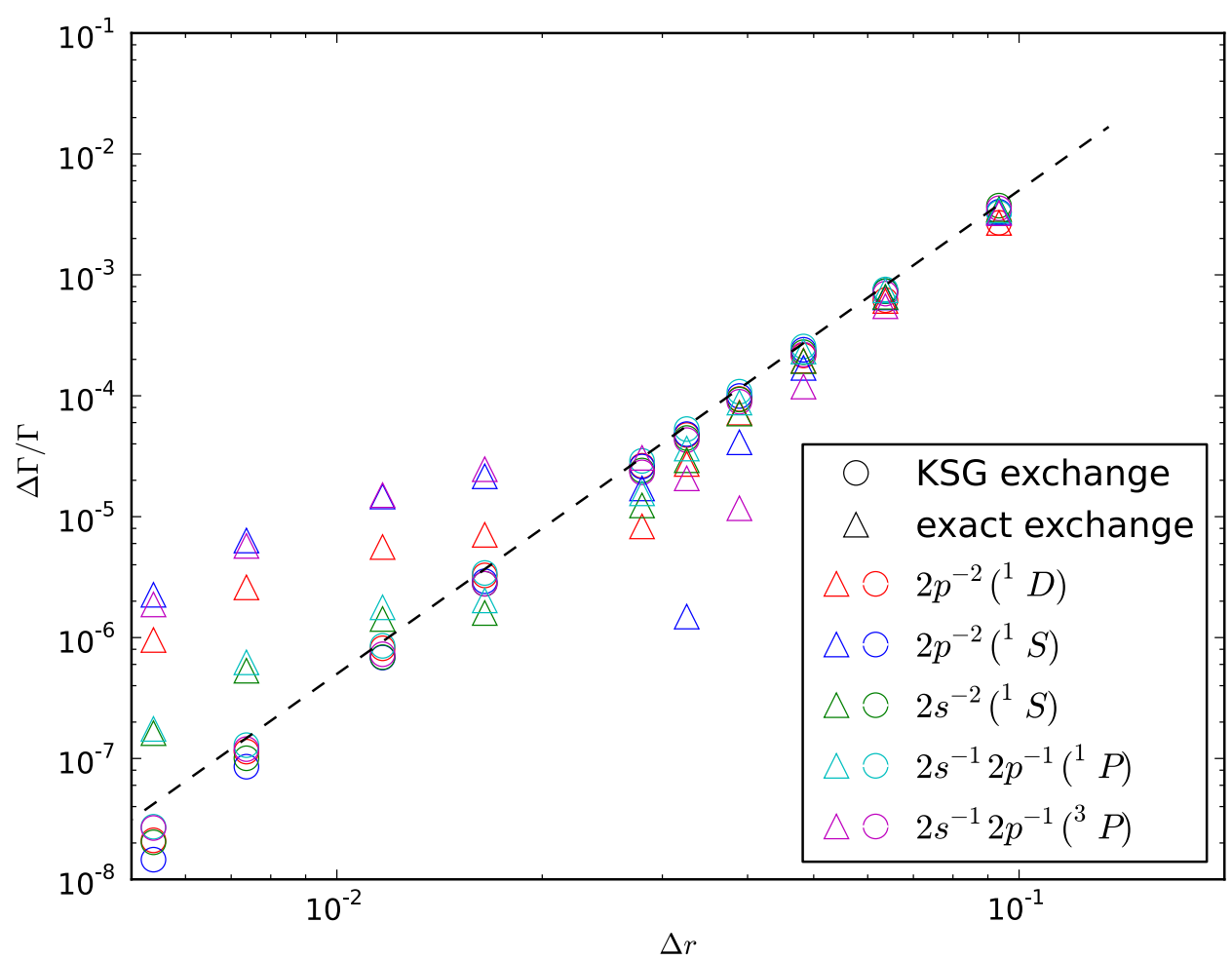

Figure 3.5: Relative error for the calculated Auger transition rates calculated with cc-pVTZ basis and (MR)CISD truncation scheme with exact and approximated exchange interaction as a function of the asymptotic grid spacing. The dotted line illustrates the $\Delta r^{4}$ power law, which is expected from the discretization error according to the Numerov scheme. The circles denote calculations with KSG exchange approximation, triangles denote calculations with exact exchange. The color denotes the respective final state.

$r \rightarrow r_{\max }$. Figure 3.5 shows the relative deviation of the calculated transition rates as a function of $\Delta r$ for both types of calculations, with exact exchange and with KSG exchange approximation. The reference solution was calculated with $N=5000$ grid points, which yields an asymptotic grid spacing of $\Delta r \simeq 0.004 a_{0}$. All calculations were performed using the CISD truncation scheme and the cc-pVTZ basis set.

As can be seen, for larger asymptotic grid spacing $\Delta r$, all the calculated transition rates follow the expected discretisation error. For $\Delta r$ below 0.03 a.u., the calculated values obtained with exact exchange deviate from the power law, and the relative error 
shows a systematic offset from the $\Delta r^{4}$ law. This behavior can be explained with additional numerical errors, which occur when obtaining the solution in an iterative scheme. Nevertheless, both calculation schemes (with exact exchange and with KSG exchange approximation) converge.

\subsubsection{Auger spectrum of neon after single and double core ionization}

To further validate the calculated transition rates, they are compared in Fig. 3.6 with the measured K-LL Auger spectrum, using values obtained with the KSG exchange approximation (see Table 3.2. . As can be seen, most features of the experimental spectrum are well represented by the calculated transition rates. The small peaks measured at 755-765 eV and at 785-795 eV are due to initial state shake-off and shake-up contributions 66.

Similar to the K-LL case, I next compare the experimentally determined KK-KLL spectrum, which occurs after double ionization of the K-shell, with the calculated transition rates and energies using the KSG exchange approximation. In Fig. 3.7 the measured spectrum by Southworth et al. 85, obtained via single-photon double ionization, is shown together with the calculated transition rates. To isolate the KK-KLL spectrum, the measured spectrum had to be subtracted from the background of the $2 p^{-2}\left({ }^{1} D\right)$ line of the K-LL Auger spectrum. Thus the spectral range below $820 \mathrm{eV}$ is missing in the experimental spectrum. As can be seen, the measured spectrum shows more structure than the calculated transitions provide. The additional peaks at $\simeq 845-865 \mathrm{eV}$ were interpreted by Southworth et al. 85] as additional state shake-up contributions in the initial $1 s^{-2}\left(1^{S}\right)$ state, in particular the additional excitations $2 p \rightarrow 3 p\left({ }^{1} S\right)$ and $2 s \rightarrow 3 s\left({ }^{3} S\right)$. These shake-up states are not relevant within the present context, and were therefore not included within the calculations performed here. They may, however, easily be considered by performing calculations using initial states, which resemble possible shake-up excitations. 


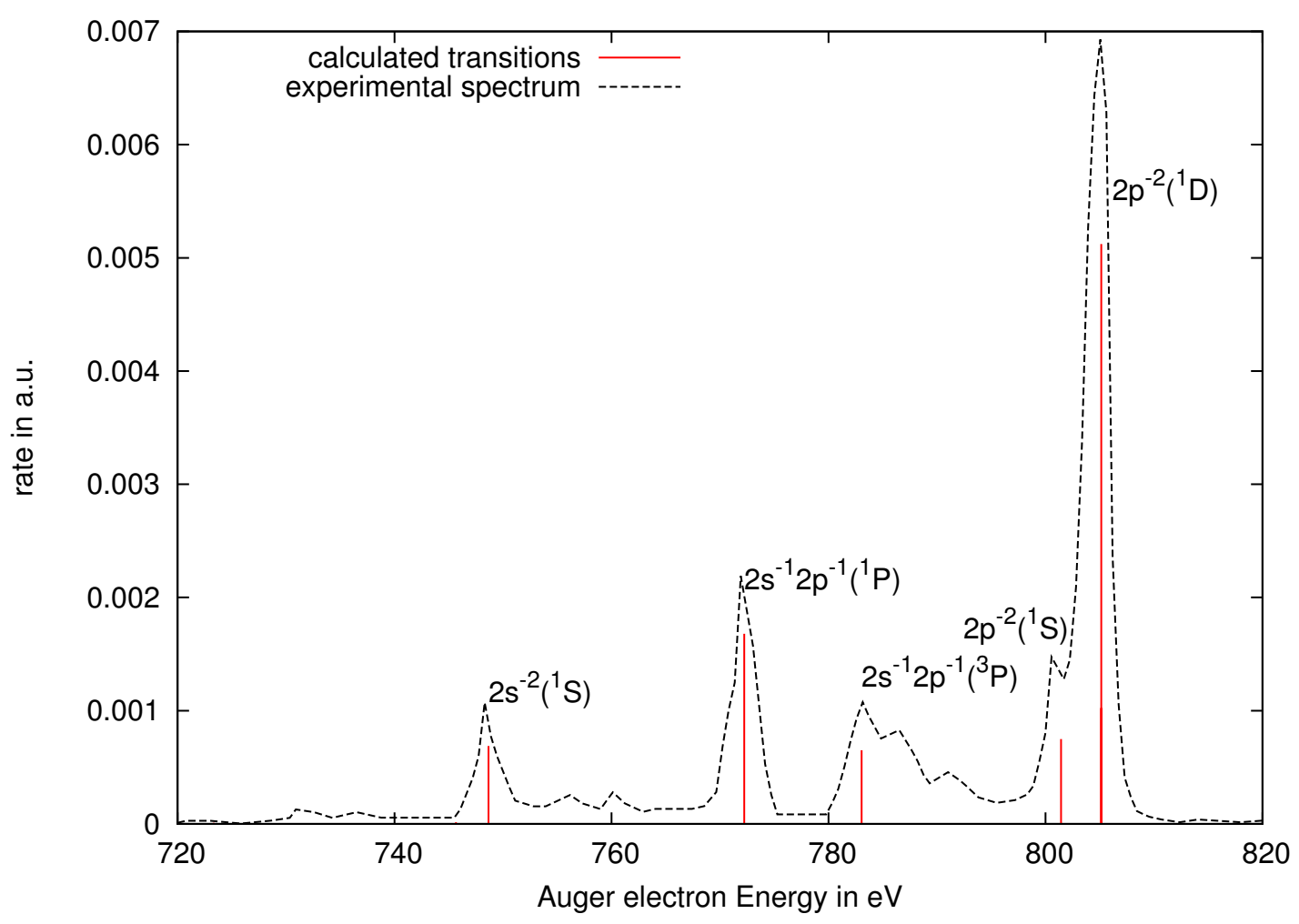

Figure 3.6: Calculated transitions compared to the measured K-LL Auger spectrum. The red vertical lines depict the calculated spectrum (cc-pVTZ basis, CISD/MRCISD, approximated exchange potential), after shifting the transition energy by $0.9 \mathrm{eV}$. The calculated transitions are labeled with the dominant hole configuration and the symmetry of the respective final states. The dashed line represents the measured spectrum taken from Ref. [85]. Most features of the experimental spectrum can be obtained from the calculation. 


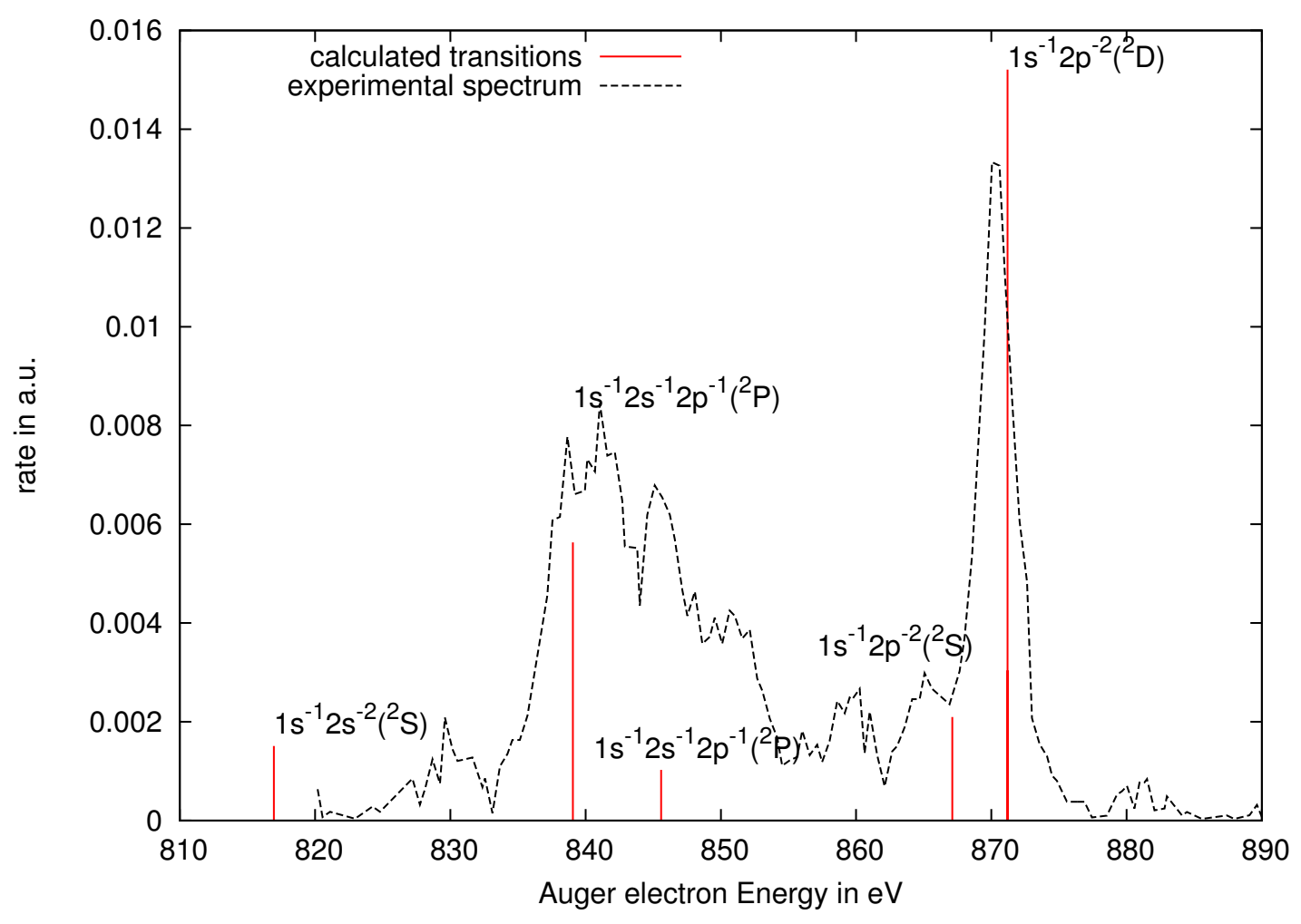

Figure 3.7: Calculated transitions compared to the measured KK-KLL Auger spectrum. The red vertical lines depict the calculated spectrum (cc-pVTZ basis, CISD/MRCISD, approximated exchange potential), after shifting the transition energy by $0.9 \mathrm{eV}$. The calculated transitions are labeled with the dominant hole configuration and the symmetry of the respective final states. The dashed line represents the measured spectrum taken from Ref. [85]. Additional peaks at $\simeq 850--870 \mathrm{eV}$ are due to shake-up contributions in the initial state. 


\section{Results}

\subsubsection{Discussion}

In this section, the procedure for calculation Auger decay rates was validated and its accuracy was systematically assessed with respect to all relevant parameters. These include the choice of basis set, the CI truncation scheme, and the radial grid size. This analysis revealed that the cc-pVTZ basis set and a (MR)CISD truncation scheme is a reasonable choice for evaluating Auger decay rates in neon and suggests that the same choice of parameters is appropriate to study other electron systems of similar size.

The comparison of the transition rates obtained by exact interaction and the KSG exchange approximation showed, that for some transitions, the KSG exchange approximation might bias the result by about $15 \%$. However, this deviation was only observed for the triplet final state, such that the total sum of decay rates was still in good agreement.

The calculated transition rates were compared with measured spectra and show good agreement for both cases, the K-LL and the KK-KLL Auger decay. In summary, the presented method to calculate Auger transitions yields reliable results for transition rates as well as for transition energies.

\subsection{Auger spectrum of water after single and double core ionization}

\subsubsection{Introduction}

In this article, we applied the method described in the previous chapter to study the evolution of a small molecule exposed to an intense X-ray pulse. As an example, we investigated the Auger spectrum of a water molecule after single and double core ionization. To study the dissociation of the water molecule and to estimate the vibrational broadening in the spectrum, we performed excited state molecular dynamics (MD) simulations, that follow the dissociative motion of the nuclei in the single and double core ionized state. From different snapshots of the MD simulations, Auger spectra were calculated and accumulated. The water molecule was chosen as a test system, because it is biologically relevant, and the limited number of electrons (10) allows for applying high level quantum chemical methods. Also, a number of previous studies on the Auger spectrum of water after single core ionization $[16,70,84,88$ allowed for a direct comparison of 


\section{Results}

both measured and calculated spectra with obtained results. The article was published in the Journal of Chemical Physics.

The main results of this study are:

- The presented approach to calculate bound-continuum transitions yields transition rates that compare well to other ab-initio approaches and experimentally determined ionization line-widths.

- The calculated Auger spectrum of a water molecule after single core ionization agrees very well with measured spectra 70 .

- In the double core ionized state, the molecule rapidly dissociates via repulsion of both protons. In contrast, the molecule remains bound in the single core ionized state .

- The strong dissociative motion in the double core ionized state blurs the Auger spectrum. Specifically, the rapid dissociation causes marked tails on the right (upshifted) side of each peak in the spectral part associated with the double K-hole decay.

- The calculated Auger decay rate of the double K-hole is significantly larger than expected from the calculated single K-hole decay rate.

\section{Individual contributions of the authors}

I have developed the CI program, the program for solving the continuum solutions and for calculating the transition rates. I have performed all the calculations on the institute's computer cluster, provided the analysis of the results, and drafted the manuscript. C. F. Burmeister helped with the conception of certain parts of the CI program. G. Groenhof helped with the calculation of the core ionized states via the Hartree-Fock method, H. Grubmüller helped with the inclusion of nuclear dynamics effects in the Auger spectrum. G. Groenhof and H. Grubmüller initiated and supervised the project and contributed to the manuscript. 


\title{
3 Results
}

\subsubsection{Article}

Reprinted with permission from The Journal of Chemical Physics, 136, 144304 (2012). Copyright 2012, American Institute of Physics.

\author{
THE JOURNAL OF CHEMICAL PHYSICS 136, 144304 (2012)
}

\section{Auger spectrum of a water molecule after single and double core ionization}

L. Inhester, ${ }^{\text {a) }}$ C. F. Burmeister, G. Groenhof, and H. Grubmüller

Max Planck Institute for Biophysical Chemistry, Am Faßberg 11, 37077 Göttingen, Germany

(Received 19 December 2011; accepted 19 March 2012; published online 9 April 2012)

The high intensity of free electron lasers opens up the possibility to perform single-shot molecule scattering experiments. However, even for small molecules, radiation damage induced by absorption of high intense $\mathrm{x}$-ray radiation is not yet fully understood. One of the striking effects which occurs under intense $\mathrm{x}$-ray illumination is the creation of double core ionized molecules in considerable quantity. To provide insight into this process, we have studied the dynamics of water molecules in single and double core ionized states by means of electronic transition rate calculations and ab initio molecular dynamics (MD) simulations. From the MD trajectories, photoionization and Auger transition rates were computed based on electronic continuum wavefunctions obtained by explicit integration of the coupled radial Schrödinger equations. These rates served to solve the master equations for the populations of the relevant electronic states. To account for the nuclear dynamics during the core hole lifetime, the calculated electron emission spectra for different molecular geometries were incoherently accumulated according to the obtained time-dependent populations, thus neglecting possible interference effects between different decay pathways. We find that, in contrast to the single core ionized water molecule, the nuclear dynamics for the double core ionized water molecule during the core hole lifetime leaves a clear fingerprint in the resulting electron emission spectra. The lifetime of the double core ionized water was found to be significantly shorter than half of the single core hole lifetime. () 2012 American Institute of Physics. [http://dx.doi.org/10.1063/1.3700233]

\section{INTRODUCTION}

Ultra intense femtosecond free electron lasers (FEL) allow one to study several new phenomena in molecules and atoms and hold the promise to obtain $\mathrm{x}$-ray scattering information from large biomolecules such as proteins at the single molecule level. ${ }^{1,2}$ Molecules exposed to intense X-ray pulses are expected to undergo severe radiation damage. ${ }^{1}$ At illumination conditions in the $\mathrm{x}$-ray regime the dominant electronic process is photoionization of core electrons into the continuum. These core ionizations trigger autoionization processes, e.g., Auger decay, which cause refilling of the core hole vacancy while emitting a secondary electron that carries away the excess energy.

Recent theoretical studies have addressed the formation of multiple core ionized electronic states by x-ray FEL radiation in atoms. ${ }^{3-5}$ These electronic states mainly result from sequential photoionization processes where the second photoionization occurs faster than the refilling of the core shell by Auger decay. Double core hole states are of particular interest in spectroscopy, as they can provide more insight into molecular structure than conventional single core spectroscopy. ${ }^{6-9}$ In experiments with intense FEL $\mathrm{x}$-ray pulses at the Linac Coherent Light Source (LCLS) significant quantities of such multiple core ionized states in neon atoms ${ }^{10}$ and nitrogen molecules ${ }^{11}$ were observed.

In addition to the pure electronic radiation damage, a second consequence of the exposure of molecules to intense

\footnotetext{
a)linhest@gwdg.de.
}

$\mathrm{x}$-ray radiation is the fast dissociative motion, the so-called Coulomb explosion, which is triggered by the fast charging of the molecule. This process has been studied by molecular dynamics force field simulations, ${ }^{1,12-14}$ in which electronic transitions are described stochastically, governed by atomic transition rates. However, because the molecular dynamics strongly depends on the ionization kinetics, accurate molecular photoionization, and Auger decay rates are desirable. From another point of view, Auger spectroscopy may provide a means to study the ionization dynamics and might give information on the fast nuclear motion. It is therefore of interest to elucidate ionization of molecules by intense $\mathrm{x}$-ray radiation and the formation of the corresponding Auger spectra with respect to the several ionization steps and the rapid nuclear motion.

One of the main challenges of calculating molecular Auger decay rates is the appropriate description of the continuum electron wavefunction, which cannot be represented by the commonly used square-integrable $\left(L^{2}\right)$ basis functions. Auger transitions for small molecules have been studied in several approaches, using (i) Stieltjes imaging, ${ }^{15}$ (ii) solving the Lippmann-Schwinger equation on a basis of Gaussiantype functions, ${ }^{16,17}$ (iii) the so-called one-center approach using atomic radial Auger integrals, ${ }^{18}$ and (iv) based on population analysis. ${ }^{19,20}$ The former two approaches rely on an asymptotical description of the continuum wavefunction with Gaussian basis functions close to the molecule, whereas the one-center approach uses atomic continuum wavefunctions. The method based on population analysis does not include the continuum electron explicitly. 


\section{Results}

Here, we calculated molecular ionization and Auger transition rates using the single center expansion (SCE) method. ${ }^{2}$ In our approach the continuum wavefunction is obtained by explicit integration of a set of coupled (static) radia Schrödinger equations, whereas the remaining bound electrons are described by usual linear combination of atomic orbitals (LCAO). This hybrid approach enabled us to accurately represent continuum wave functions while taking advantage of efficient $L^{2}$ basis sets for the bound orbitals.

Using the obtained molecular ionization and Auger decay rates, time-dependent populations of the single and double core ionized states were calculated, similarly to previous approaches for atoms. ${ }^{3-5}$ To also include effects of nuclear motion on the Auger spectrum within the core hole life time, we incoherently summed up instantaneous Auger spectra for different molecular geometries, obtained by classically propagating the nuclei positions with forces calculated "on the fly" for the core ionized electronic states. Such approach, already applied in previous calculations, ${ }^{22,23}$ avoids the explicit computation of the many involved potential energy surfaces but neglects possible interference effects on the spectrum. Other approaches, which address effects of nuclear motion in a coherent way and, thereby, are able to address vibrational features of the spectrum, rely on pre-calculated potential energy surfaces. For examples, Eroms et al. ${ }^{24}$ used the multi-configurational time-dependent Hartree technique to propagate the nuclear wave packets for the resonant Auge spectrum of water. Bao et al. ${ }^{25}$ presented a calculation of the normal Auger spectrum of the oxygen molecule based on the Kramers-Heisenberg formula

As a model system we considered the Auger spectrum of a singly and doubly core ionized water molecule. While the single core Auger spectrum $(K-L L)$ of water has been extensively studied, ${ }^{15,23,26}$ we are not aware of any studies of its double core Auger spectra $(K K-K L L)$. Our results confirm that the nuclear motion has little effect on the Auger spectrum during the few femtoseconds of the core hole lifetime for single core ionized water. ${ }^{23}$ Strikingly, however, the nuclear motion of double core ionized water was found to markedly affect the Auger spectrum due to fast dissociation dynamics.

The outline of the paper is as follows. Our approach to determine ionization transition rates is described in Sec. II. Section III describes the computational details of the calculations. Results and conclusion are presented in Secs. IV and V.

\section{CALCULATION OF ELECTRONIC IONIZATION TRANSITIONS RATES}

Calculation of ionization rates requires the description of initial $\left|\psi_{\text {ini }}\right\rangle$ and final $\left|\psi_{\text {fin }}\right\rangle$ electronic wave functions. To clarify the notation, we first describe in Subsection II A how the final electronic states $\left|\psi_{\text {fin }}\right\rangle$ are constructed from a molecular bound part and a continuum part. In Subsections II B and II $\mathrm{C}$ we describe how photoionization cross sections and Auger decay rates are obtained.

\section{A. Construction of the final electronic state}

A total final electronic state $\left|\psi_{\text {fin }}\right\rangle$ after ionization is constructed by combining a multi-electron bound part $\left|\tilde{\psi}_{\text {fin }}\right\rangle$ and single electron part described by the continuum electron wave function $\phi_{k, \sigma}(\mathbf{r})$ with energy $\epsilon=k^{2} / 2$ and spin $\sigma$. Following spin addition rules ${ }^{27}$ the state is given by

$$
\left|\psi_{\mathrm{fin}, M_{S}=1 / 2}^{(1) S=1 / 2}\right\rangle=c_{k, \alpha}^{\dagger}\left|\tilde{\psi}_{\mathrm{fin}, M_{S}=0}^{S=0}\right\rangle,
$$

$$
\left|\psi_{\mathrm{fin}, M_{S}=1 / 2}^{(2) S=1 / 2}\right\rangle=\frac{1}{\sqrt{3}}\left(-c_{k, \alpha}^{\dagger}\left|\tilde{\psi}_{\mathrm{fin}, M_{S}=0}^{S=1}\right\rangle+\sqrt{2} c_{k, \beta}^{\dagger}\left|\tilde{\psi}_{\mathrm{fin}, M_{S}=1}^{S=1}\right\rangle\right),
$$

for doublet states and

$$
\left|\psi_{\mathrm{fin}, M_{S}=0}^{S=0}\right\rangle=\frac{1}{\sqrt{2}}\left(-c_{k, \alpha}^{\dagger}\left|\tilde{\psi}_{\mathrm{fin}, M_{S}=-1 / 2}^{S=1 / 2}\right\rangle+c_{k, \beta}^{\dagger}\left|\tilde{\psi}_{\mathrm{fin}, M_{S}=1 / 2}^{S=1 / 2}\right\rangle,\right.
$$

for singlet states. The additional indices $S$ and $M_{S}$ describe total spin quantum numbers ${ }^{28}$ and $c_{k, \sigma}^{\dagger}$ is the creation operator for a continuum electron with wavefunction $\phi_{k, \sigma}(\mathbf{r})$.

For the evaluation of transition rates given by first-order perturbation theory, matrix elements of an operator $O$ between initial $\left|\psi_{\text {ini, } M_{S}}^{S}\right\rangle$ and final states $\left|\psi_{\text {fin, } M_{S}}^{S}\right\rangle$ have to be calculated. Assuming $O$ commutes with spin $S$, expressions for final states $\left|\psi_{\text {fin, } M_{S}=1 / 2}^{(2)}\right\rangle$ and $\left|\psi_{\text {fin, } M_{S}=0}^{S=0}\right\rangle$ can be simplified to ${ }^{29}$

$$
\begin{aligned}
& \left|\left\langle\psi_{\mathrm{ini}, M_{S}=1 / 2}^{S=1 / 2}|O| \psi_{\mathrm{fin}, M_{S}=1 / 2}^{(2) S=1 / 2}\right\rangle\right|^{2} \\
& \quad=3\left|\left\langle\psi_{\mathrm{ini}, M_{S}=1 / 2}^{S=1 / 2}|O| c_{k, \alpha}^{\dagger} \tilde{\psi}_{\mathrm{fin}, M_{S}=0}^{S S=1}\right\rangle\right|^{2},
\end{aligned}
$$

$$
\begin{aligned}
& \left|\left\langle\psi_{\mathrm{ini}, M_{S}=0}^{S=0}|O| \psi_{\mathrm{fin}, M_{S}=0}^{S=0}\right\rangle\right|^{2} \\
& \quad=2\left|\left\langle\psi_{\mathrm{ini}, M_{S}=0}^{S=0}|O| c_{k, \alpha}^{\dagger} \tilde{\psi}_{\mathrm{fin}, M_{S}=-1 / 2}^{S=1 / 2}\right\rangle\right|^{2} .
\end{aligned}
$$

The bound part $\left|\tilde{\psi}_{f i n, M_{S}}^{S}\right\rangle$ of the final electronic wavefunction can be represented by the usual linear combination of atomic orbitals (LCAO), while the description of the continuum wavefunction $\phi_{k, \sigma}$ requires a continuum representation. Here we represent $\phi_{k, \sigma}$ in a single center expansion ${ }^{21}$ given by

$$
\phi_{k, \sigma}(\mathbf{r})=\sum_{l m} \frac{P_{l m}^{k}(r)}{r} Y_{l m}(\theta, \phi),
$$

where $Y_{l m}(\theta, \phi)$ are spherical harmonics, and the $P_{l m}^{k}(r)$ are a set of radial wave functions, which solve the set of coupled radial Schrödinger equations ${ }^{21}$

$$
\frac{d^{2}}{d r^{2}} P_{l m}^{k}(r)+\sum_{l^{\prime} m^{\prime}} M_{l m, l^{\prime} m^{\prime}}(r) P_{l^{\prime} m^{\prime}}^{k}(r)=0,
$$




\section{Results}

with

$$
\begin{aligned}
M_{l m, l^{\prime} m^{\prime}}(r):= & \delta_{l^{\prime}, l} \delta_{m^{\prime}, m}\left(\frac{-l(l+1)}{r^{2}}+2 \epsilon\right) \\
& +2 \sum_{l^{\prime \prime} m^{\prime \prime}} v_{l^{\prime \prime} m^{\prime \prime}}(r) \int d \Omega Y_{l m}^{*}(\theta, \phi) \\
& \times Y_{l^{\prime \prime} m^{\prime \prime}}(\theta, \phi) Y_{l^{\prime} m^{\prime}}(\theta, \phi) .
\end{aligned}
$$

Here, $\int d \Omega$ describes integration over the solid angle and $v_{l m}(r)$ are the radial parts of the SCE of the potential $V(\mathbf{r})$ where

$$
\begin{aligned}
V(\mathbf{r}) & =\sum_{l m} \frac{v_{l m}(r)}{r} Y_{l m}(\theta, \phi)=V_{\mathrm{ne}}(\mathbf{r})+V_{\mathrm{ee}}(\mathbf{r}) \\
& =\sum_{l m} \frac{v_{l m}^{n e}(r)+v_{l m}^{e e}(r)}{r} Y_{l m}(\theta, \phi) .
\end{aligned}
$$

$V_{\text {ne }}(\mathbf{r})$ is the nuclear potential of the molecule and $V_{\text {ee }}(\mathbf{r})$ represents the interaction of the continuum electron with the remaining bound electrons. For the spherical nuclear coordinates $R_{n}, \theta_{n}, \phi_{n}$ of nucleus $n=1 \ldots N$, the radial parts $v_{l m}^{n e}(r)$ of the nuclear potential are given by

$$
v_{l m}^{n e}(r)=\sum_{n}-Z_{n} r \frac{r_{<}^{l}}{r_{>}^{l+1}} Y_{l m}^{*}\left(\theta_{n}, \phi_{n}\right),
$$

with $r_{<}=\min \left(r, R_{n}\right)$ and $r_{>}=\max \left(r, R_{n}\right)$ and $Z_{n}$ being the charge of nucleus $n$. The electron-electron interaction $V_{\mathrm{ee}}(\mathbf{r})$ is determined by the electrostatic potential of the charge density $\tilde{\rho}(\mathbf{r})$ of the electrons in the bound part $\left|\tilde{\psi}_{\text {fin }}\right\rangle$,

$$
J(\mathbf{r})=\int d \mathbf{r}^{\prime} \frac{\tilde{\rho}\left(\mathbf{r}^{\prime}\right)}{\left|\mathbf{r}-\mathbf{r}^{\prime}\right|},
$$

and the shorter ranged exchange part. We used the KSG (Ref. 30) exchange potential,

$$
V_{\mathrm{XC}}[\tilde{\rho}(\mathbf{r})]=-\left(\frac{3}{\pi} \tilde{\rho}(\mathbf{r})\right)^{\frac{1}{3}},
$$

to model the exchange interaction, which renders Eq. (7) as a homogenous differential equation. To further simplify the calculations, the non-spherical symmetric parts $(l \neq 0)$ of the electron density $\tilde{\rho}(\mathbf{r})$ in the exchange potential (Eq. (12)) are approximated by first-order Taylor expansion

$$
\begin{aligned}
V_{\mathrm{XC}}[\tilde{\rho}(\mathbf{r})]= & -\left(\frac{3}{\pi} \frac{\tilde{\rho}_{00}(r)}{r} Y_{00}\right)^{\frac{1}{3}}\left[1+\frac{1}{3} \sum_{l \neq 0, m} \frac{\tilde{\rho}_{l m}(r) Y_{l m}(\theta, \phi)}{\tilde{\rho}_{00}(r) Y_{00}}\right. \\
& \left.+\mathcal{O}\left(\sum_{l \neq 0, m} \frac{\tilde{\rho}_{l m}(r) Y_{l m}(\theta, \phi)}{\tilde{\rho}_{00}(r) Y_{00}}\right)^{2}\right]
\end{aligned}
$$

where $\tilde{\rho}_{l m}(r)$ are the radial parts of the SCE of the electron density

$$
\tilde{\rho}(\mathbf{r})=\sum_{l m} \frac{\tilde{\rho}_{l m}(r)}{r} Y_{l m}(\theta, \phi) .
$$

The radial parts $v_{l m}^{e e}(r)$ of the electron-electron interactions in Eq. (9) are finally given by the SCE of Coulomb potential $J(\mathbf{r})$ and the electron density $\tilde{\rho}(\mathbf{r})$

$$
\begin{aligned}
r \cdot v_{l m}^{e e}(r) \simeq & \int d \Omega Y_{l m}^{*}(\theta, \phi) J(\mathbf{r})+\frac{-1}{Y_{00}}\left(\frac{3}{\pi} \frac{\tilde{\rho}_{00}(r)}{r} Y_{00}\right)^{\frac{1}{3}} \\
& \times \begin{cases}1 & \text { for } l=0 \\
\tilde{\rho}_{l m}(r) /\left(3 \tilde{\rho}_{00}(r)\right) & \text { else. }\end{cases}
\end{aligned}
$$

Subsequently, the energy-degenerated solutions of Eq. (7), $P_{l m}^{\prime k, L M}(r)$, are labeled by the additional index tuple $L M$. They are required to fulfill the boundary conditions

$$
P_{l m}^{\prime k, L M}(r \rightarrow 0)=0,
$$

$$
P_{l m}^{\prime k, L M}(r \rightarrow \infty)=\sqrt{\frac{2}{\pi k}}\left(\delta_{L M, l m} F_{l}(k r)+R_{L M, l m} G_{l}(k r)\right) .
$$

$F_{l}(k r)$ and $G_{l}(k r)$ are the regular and irregular Coulomb functions and $R_{L M I m}$ are elements of an hermitian matrix $\mathbf{R}$ determined by the asymptotic behavior of the solutions. ${ }^{2}$

The above mentioned boundary conditions do not provide an energy-normalized solution, as required for correct transition rates. Hence, energy normalization is achieved by the linear combination ${ }^{31}$

$$
P_{l m}^{k, L M}(r)=\sum_{L^{\prime} M^{\prime}} \frac{1}{\sqrt{1+\lambda_{L M}}} U_{L M, L^{\prime} M^{\prime}} P_{l m}^{\prime k, L^{\prime} M^{\prime}},
$$

where the columns of $\mathbf{U}$ and $\lambda_{L M}$ are eigenvectors and eigenvalues of the Matrix $\mathbf{R}$, respectively.

Note, that here the bound electrons are considered to be not affected by the continuum electron, thus the bound electron part can be calculated independently. To evaluate electronic transition rates, electron integrals between bound an continuum electrons have to be calculated. For the purpose of calculating these quantities within the SCE, also the bound orbitals are expanded into the SCE as in Eq. (6).

\section{B. Photoionization}

Following first-order perturbation theory, the photoionization cross sections $\sigma_{\mathrm{ini}} \rightarrow$ fin in length gauge is proportional to the dipole matrix elements between the initial $\left|\psi_{\text {ini }}\right\rangle$ and final $\left|\psi_{\text {fin }}\right\rangle$ electronic states, ${ }^{32}$

$$
\sigma_{\text {ini } \rightarrow \text { fin }}=4 \alpha \pi^{2} \omega\left|\left\langle\psi_{\text {ini }}|\mathbf{s} \cdot \mathbf{d}| \psi_{\text {fin }}\right\rangle\right|^{2},
$$

where $\alpha \simeq 1 / 137$ is the fine-structure constant, $\omega$ is the photon energy, and $\mathbf{s}$ is the electric polarization vector of the electromagnetic wave. The elements of the transition dipole moment d are expressed in the SCE by ${ }^{5}$

$$
\begin{aligned}
d_{M}= & c_{i}^{\dagger} c_{j} \sqrt{\frac{4 \pi}{3}} \sum_{l m l^{\prime} m^{\prime}} \int_{0}^{\infty} d r P_{l m}^{i *}(r) r P_{l m}^{j}(r) \\
& \times \int d \Omega Y_{l m}(\theta, \phi) Y_{l^{\prime} m^{\prime}}^{*}(\theta, \phi) Y_{1 M}^{*}(\theta, \phi),
\end{aligned}
$$

where $P_{l m}^{i}(r)$ and $P_{l^{\prime} m^{\prime}}^{j}(r)$ represent radial parts of the respective bound and continuum electron wave functions and $c_{i}^{\dagger}, c_{j}$ 


\section{Results}

are the corresponding creation/annihilation operators, respectively. Averaging over all molecular orientations, yields

$$
\sigma_{\text {ini } \rightarrow \text { fin }}=\frac{4}{3} \alpha \pi^{2} \omega \sum_{M=-1,0,1}\left|\left\langle\psi_{\text {ini }}\left|d_{M}\right| \psi_{\text {fin }}\right\rangle\right|^{2} .
$$

The photoionization transition rate is given by

$$
\Gamma_{\text {ini } \rightarrow \text { fin }}^{\text {Photo }}=\sigma_{\text {ini } \rightarrow \text { fin }} \cdot F(t),
$$

where $F(t)$ is the time-dependent photon flux. The total photoionization cross section $\sigma_{\text {ini }}$ reads

$$
\sigma_{\text {ini }}=\sum_{\text {fin }} \sigma_{\text {ini } \rightarrow \text { fin }} .
$$

The above summation involves different continuum solutions $(L M)$ as well as different electronic bound parts $\left(\left|\tilde{\psi}_{\text {fin }}\right\rangle\right)$.

\section{Auger transition}

The transition rate for Auger decay $\Gamma_{\text {ini } \rightarrow \text { fin }}^{\text {Auger }}$ from firstorder perturbation theory is given by

$$
\Gamma_{\text {ini } \rightarrow \text { fin }}^{\text {Auger }}=2 \pi\left|\left\langle\psi_{\text {fin }}\left|H-E_{\text {ini }}\right| \psi_{\text {ini }}\right\rangle\right|^{2} .
$$

Assuming vanishing state overlap $\left\langle\psi_{\text {fin }} \mid \psi_{\text {ini }}\right\rangle=0$, the Auger transition rates are given by matrix elements of the electronic Hamiltonian, ${ }^{33}$

$$
\begin{aligned}
& \left\langle\psi_{\mathrm{fin}}\left|H-E_{\mathrm{ini}}\right| \psi_{\mathrm{ini}}\right\rangle \\
& =\left\langle\psi_{\mathrm{fin}}\left|\left(\sum_{i j} c_{i}^{\dagger} c_{j} h_{i j}+\frac{1}{2} \sum_{i j k l} c_{i}^{\dagger} c_{j}^{\dagger} c_{l} c_{k}\langle i j \mid k l\rangle\right)\right| \psi_{\mathrm{ini}}\right\rangle
\end{aligned}
$$

The above two- and one-electron integrals are readily evaluated in the SCE representation,

$$
\begin{aligned}
h_{i j}= & \delta_{\sigma_{i}, \sigma_{j}} \int d \mathbf{r} \phi_{i}^{*}(\mathbf{r})\left(-\frac{\Delta}{2}+V_{n e}(\mathbf{r})\right) \phi_{j}(\mathbf{r}) \\
= & \delta_{\sigma_{i}, \sigma_{j}} \int_{0}^{\infty} d r\left(\sum_{l m} \frac{1}{2} \frac{d P_{l m}^{i *}(r)}{d r} \frac{d P_{l m}^{j}(r)}{d r}\right. \\
& +\sum_{\substack{l m \\
l^{\prime} m^{\prime} l^{\prime \prime} m^{\prime \prime}}} \frac{P_{l m}^{i *}(r) v_{n e, l^{\prime} m^{\prime}}(r) P_{l^{\prime \prime} m^{\prime \prime}}^{j}(r)}{r} \\
& \left.\times \int d \Omega Y_{l m}^{*}(\theta, \phi) Y_{l^{\prime} m^{\prime}}(\theta, \phi) Y_{l^{\prime \prime} m^{\prime \prime}}(\theta, \phi)\right),
\end{aligned}
$$

and

$$
\langle i j \mid k l\rangle:=\delta_{\sigma_{i}, \sigma_{k}} \delta_{\sigma_{j}, \sigma_{l}}(i k \mid j l),
$$

$$
\begin{aligned}
(i k \mid j l)= & \int d^{3} r_{1} \int d^{3} r_{2} \phi_{i}^{*}\left(\mathbf{r}_{1}\right) \phi_{k}\left(\mathbf{r}_{1}\right) \frac{1}{r_{12}} \phi_{j}^{*}\left(\mathbf{r}_{2}\right) \phi_{l}\left(\mathbf{r}_{2}\right) \\
= & \sum_{\substack{l m \\
l^{\prime} m^{\prime} l^{\prime \prime} m^{\prime \prime}}} \int_{0}^{\infty} d r y_{l m}^{i k}(r) P_{l^{\prime} m^{\prime}}^{j *}(r) P_{l^{\prime \prime} m^{\prime \prime}}^{l}(r) \\
& \times \int d \Omega Y_{l m}^{*}(\theta, \phi) Y_{l^{\prime} m^{\prime}}^{*}(\theta, \phi) Y_{l^{\prime \prime} m^{\prime \prime}}(\theta, \phi)
\end{aligned}
$$

$$
\begin{aligned}
y_{l m}^{i k}(r):= & \sum_{\substack{l^{\prime} m^{\prime} \\
l^{\prime \prime} m^{\prime \prime}}} \int_{0}^{\infty} d r^{\prime} \frac{4 \pi P_{l^{\prime} m^{\prime}}^{i *}(r) P_{l^{\prime \prime} m^{\prime \prime}}^{k}(r)}{2 l+1} \frac{r_{<}^{l}}{r_{>}^{l+1}} \\
& \times \int d \Omega Y_{l m}(\theta, \phi) Y_{l^{\prime} m^{\prime}}^{*}(\theta, \phi) Y_{l^{\prime \prime} m^{\prime \prime}}(\theta, \phi) .
\end{aligned}
$$

Here, $\sigma_{i}$ is the spin of spin orbital $\phi_{i, \sigma_{i}}, r_{<}=\min \left(r, r^{\prime}\right)$ and $r_{>}=\max \left(r, r^{\prime}\right)$. Neglecting other relaxation effects such as fluorescence-which is small for the light nuclei considered here- the total lifetime $\tau$ of the initial state is given by

$$
\tau=1 / \Gamma_{\mathrm{ini}}^{\text {Auger }}=1 / \sum_{\mathrm{fin}} \Gamma_{\mathrm{ini} \rightarrow \text { fin }}^{\text {Auger }},
$$

where $\Gamma_{\mathrm{ini}}^{\text {Auger }}$ is the total transition rate of the initial state. Again summation index "fin" describes the complete relaxation channel given by continuum solution $(L M)$ and bound part $\left(\left|\tilde{\psi}_{\text {fin }}\right\rangle\right)$.

\section{COMPUTATIONAL DETAILS}

\section{A. Single center expansion}

Dunning's $c c-p V T Z$ basis set ${ }^{34}$ was used to represent bound molecular orbitals (MOs) in all computations. For each ionization and Auger decay step, molecular orbitals were calculated by a restricted (open shell) Hartree Fock procedure $(\mathrm{R}(\mathrm{O}) \mathrm{HF})$ optimized for the initial electronic state. Thus, we calculated MOs for the neutral and double core ionized state by RHF, respectively, and MOs for the single core ionized state with ROHF. The self-consistent field optimization for core ionized states was carried using a modified PSI3 quantum package ${ }^{35}$ as described elsewhere. ${ }^{36}$ This procedure is known to account for most of the core electron vacancy induced orbital relaxation effects. ${ }^{37}$

From the resulting MOs, the SCE of each orbital $\phi^{i}(\mathbf{r})$ (Eq. (6)) has the radial parts

$$
P_{l m}^{i}(r)=r \int d \Omega \phi^{i}(\mathbf{r}) Y_{l m}^{*}(\theta, \phi) .
$$

These radial parts as well as the radial parts of their electrostatic potential and density were numerically calculated using Gaussian-Legendre integration with $20 \times 20$ integration points in angle space. Because all of these quantities are real, real valued tesseral spherical harmonics ${ }^{38}$ instead of the usual complex valued spherical harmonics were used to reduce the computational cost. Angular integrals over three spherical harmonics, also known as Gaunt coefficients, are readily calculated by evaluation of Wigner $3-j$ symbols. ${ }^{39}$

For the radial coordinate non-equidistant radial grid points $r$ were used ${ }^{21}$ implicitly determined by

$$
\rho(r)=\alpha r+\beta \ln r+\sum_{n} \arctan \frac{r-R_{n}}{\gamma} .
$$

Here $R_{n}$ are the distances of atom $n$ to the center of the ex pansion, and variable $\rho$ is discretized on an equidistant grid. The number of radial grid points was 1500 , the largest radial grid point was set to $r=20$ a.u., and the center for the expansion was chosen at the position of the oxygen atom, which allowed the SCE to limit to angular quantum numbers $l \leq 5$, 
resulting in 36 different $(\mathrm{lm})$ tuples. These parameters turned out to represent the molecular orbitals and relevant continuum wavefunctions of the water molecule sufficiently accurate and thus have been used for all subsequent calculations.

\section{B. Configurational mixing}

We applied the frozen orbital approximation, i.e., initial and final electronic states were represented by the same orbital set. In particular, orbitals optimized for the initial state by the $\mathrm{R}(\mathrm{O}) \mathrm{HF}$ calculation mentioned above were used. The molecular orbital integrals, obtained from the PSI3 quantum package, ${ }^{35}$ were used to perform spin adapted multi-reference configuration interaction (MRCI) calculations for the final bound electronic states $\left|\tilde{\psi}_{\text {fin }, M_{S}}^{S}\right\rangle$ and single reference configuration interaction (CI) calculations ${ }^{40}$ for the initial states $\left|\tilde{\psi}_{\text {ini, } M_{S}}^{S}\right\rangle$. As references for the final state we have chosen the initial state reference with all possible combinations of one additional vacancy (for photoionization) or two valence electrons removed and a re-occupied core orbital (for Auger decay). From the reference occupations configuration state functions (CSFs) were built considering single excitations within the full MOs space and double excitations up to the 20th MO. This truncation of the CI space was used for both single and the multi-reference calculations, leading to a number of 2297-5852 CSFs for the initial states (single reference CI) and 8125-18220 CSFs for the final state (MRCI). From these calculations only solution vectors with significant contributions in the references (norm of projection into reference subspace $>0.01$ ) were used for subsequent calculations. For these solution vectors the SCE of the electrostatic potential and the electron density of the respective final electronic state was obtained as a linear combination of the electrostatic potential and electron density of the MOs.

\section{Integration of the continuum wavefunction}

For the relevant CI vectors, the 36 solutions for the continuum wavefunctions in the potential of the molecule of the specific final electronic state $\left|\tilde{\psi}_{\text {fin }}^{S}\right\rangle$ were integrated according to Eq. (7) for the given boundary conditions (Eq. (17)) using the vector sweep integration method adopted from Ref. 21. The continuum normalization was carried out by diagonalizing the obtained $\mathbf{R}$ matrix as described by Eq. (18).

\section{Transitions}

Photoionization cross sections and Auger decay transition rates were calculated by evaluating Eqs. (21) and (25) using Simpson's rule. In particular, for the two electron integrals a system of coupled differential equation $\mathrm{s}^{21,41}$ was solved for $r \cdot y_{l m}^{i k}(r)$ and then Eq. (28) was integrated by Simpson's rule, as had been described for atoms. ${ }^{42}$ As different angular continuum channels were not distinguished here, transition rates for different continuum solutions $(L M)$ were finally summed up.

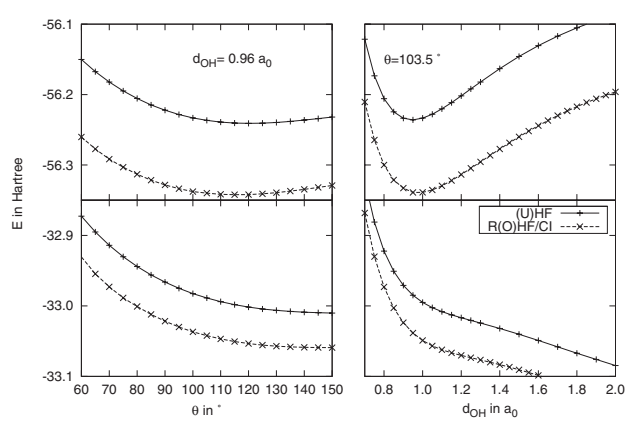

FIG. 1. Cuts through the potential energy surfaces for single (top) and double core (bottom) ionized water. (Left) Both hydrogen atoms are at equilibrium distance $d_{\mathrm{OH}}=0.96 a_{0}$ to the oxygen for different $\mathrm{HOH}$ angles $\Theta$. (Right) One hydrogen atom is fixed at $d_{\mathrm{OH}}=0.96 a_{0}$ and the other is at variable distances to the oxygen atom, while $\Theta$ is at equilibrium value of $103.5^{\circ}$.

\section{E. Molecular dynamics calculations}

All calculations of Auger decay rates were performed for a set of molecular geometries, obtained by ab initio molecular dynamics (MD) simulation. The nuclei were propagated using the Beeman integration scheme ${ }^{43}$ with a $0.1 \mathrm{fs}$ time step in the single and double core ionized state. Energy gradients were calculated with GAUSSIAN 09 (Ref. 44) from unrestricted Hartree Fock (UHF) calculations for the single core ionized state and restricted Hartree Fock (RHF) calculations for the double core ionized state. Convergence of the selfconsistent field procedure to the desired open core shell states was achieved by choosing an initial guess based on neutral optimized orbitals with single or unoccupied core orbital, respectively. To assess the accuracy of the (U)HF method used to generate the trajectories, we compared two sections of the potential energy surface obtained by the single determinant (U)HF method with that obtained by the CI method described in Sec. II B. As seen in Fig. 1, very similar curves are obtained, apart from a nearly constant offset. Therefore, we consider the gradients at (U)HF level to be sufficiently accurate to describe the nuclear dynamics after core ionizations.

\section{F. Initial conditions}

The Auger spectrum of water is dominated by FranckCondon broadening due to the very steep potential energy surfaces of the final electronic states. To estimate this broadening (as described further below), multiple MD trajectories were calculated with initial conditions at the $1 \sigma$ standard deviation of the ground state Wigner distribution. To that aim, neutral ground state optimization and vibrational mode calculations ${ }^{40}$ using harmonic approximation were performed with GAUSSIAN 09 (Ref. 44) on the MP2 level. From the optimized ground state geometry six different initial conditions with zero velocities were generated by varying the geometry in positive and negative directions along each of the three normal modes by the standard deviation $\sigma$ of the vibrational ground state distribution. Six further initial conditions were 


\section{Results}

generated from the optimized geometry with initial velocities in positive and negative directions along the vibrational normal modes. These velocities were chosen to match the standard deviation $\sigma$ of the vibrational ground state velocity distribution. Together with the optimized geometry with zero velocity, a total set of 13 sets of initial conditions were thus obtained.

\section{G. Spectrum}

For each of the 13 initial conditions, molecular dynamics simulations in the single core ionized state were started. Rather than estimating the Franck Condon broadening of the Auger lines from a weighted average over many Wignerdistributed trajectories (which would require a considerable number of trajectories to achieve sufficient sampling), the variance of the assumed Gaussian line profile of the dominan Auger transitions was estimated from the differences $\Delta \epsilon(t)$ in the Auger transition energies between the "central" trajectory (started from the optimized geometry with zero initial velocities) and the 12 "satellite" trajectories (started from altered initial conditions) as

$$
\begin{aligned}
\sigma^{2}(t)= & \sigma_{\exp }^{2}+\sigma_{\text {lifetime }}^{2}+\sum_{i=1}^{3} \frac{\Delta \epsilon_{i,+x}^{2}(t)+\Delta \epsilon_{i,-x}^{2}(t)}{2} \\
& +\frac{\Delta \epsilon_{i,+v}^{2}(t)+\Delta \epsilon_{i,-v}^{2}(t)}{2} .
\end{aligned}
$$

This computationally more efficient estimate rests on the assumption that the Gaussian shape of the nuclei wave packet is approximately maintained during the short simulation time. More precisely, we assume that the peak of the wave-packet remains sufficiently close to the "central" trajectory, and the "satellite" trajectories remain on average sufficiently close to the surrounding $1 \sigma$ hypersurface, such that the width of the wave packet can be estimated from their average distance to the "central" trajectory. Additionally, we assume that within the phase space region covered by the wave packet, the transition energy is sufficiently linear in the atomic coordinates and the individual transition rates are constant. Visual inspection of the trajectories showed that these conditions are satisfied. This allowed us to restrict the computation of Auger transition rates to the "central" trajectory, while for the "satellite" trajectories only transition energies needed to be calculated.

In Eq. (33) $\Delta \epsilon_{i, \pm x}(t)$ denotes the difference of the transition energy between the "central" and the "satellite" trajectory, started with geometries modified along the vibrational mode $i$. Similarly, $\Delta \epsilon_{i, \pm v}(t)$ denotes the difference of the transition energy between the "central" and the "satellite" trajectory, started with velocities modified along vibrational mode $i$. Additionally, the effect due to limited experimental resolution and due to line broadening was included, with $\sigma_{\mathrm{ex}}$ $=0.17 \mathrm{eV}\left(0.4 \mathrm{eV}\right.$ FWHM (Ref. 45)), and $\sigma_{\text {lifetime }}$ estimated from the decay rates calculated in Sec. IV B.

To follow the evolution of double core ionized water after a period of nuclear dynamics in the single core ionized state, additional simulations of the double core ionized state

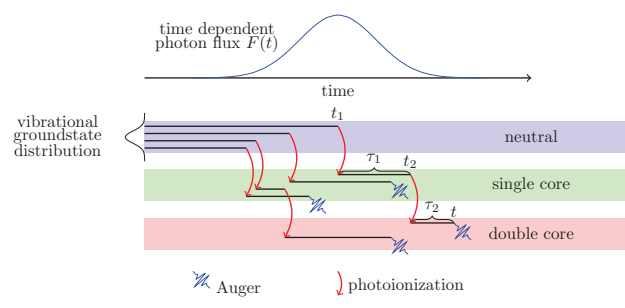

FIG. 2. Illustration of different ionization pathways. The total Auger spectrum is obtained from a superposition of spectra resulting from different trajectories along the neutral, single core ionized, and double core ionized states. Here, as an example, 4 pathways contributing to the single and the double core Auger spectrum are illustrated.

were started from selected snapshots of the single core ionized state trajectories ("central" and "satellite") at $0,1, \ldots 9 \mathrm{fs}$, thus resulting in a total of 130 double core trajectories. The simulation time for each of the concatenated single and double core trajectories was limited to $10 \mathrm{fs}$, and the simulation time in the double core ionized state was limited to $7 \mathrm{fs}$.

Figure 2 illustrates how the spectra were composed of different trajectories using different pathways through the single and double core ionized states. The accumulated single and double core hole Auger spectra, $S_{1}$ and $S_{2}$, were calculated considering all possible pathways by

$$
S_{1}=\int_{-\infty}^{\infty} d t \int_{-\infty}^{t} d t_{1} p_{1}\left(t, t_{1}\right) s_{1}\left(t-t_{1}\right)
$$

$S_{2}=\int_{-\infty}^{\infty} d t \int_{-\infty}^{t} d t_{2} \int_{-\infty}^{t_{2}} d t_{1} p_{2}\left(t, t_{1}, t_{2}\right) s_{2}\left(t-t_{1}, t-t_{1}-t_{2}\right)$,

where $s_{1}\left(\tau_{1}\right)$ is the time-dependent single core Auger spectrum obtained as instantaneous spectrum from the geometry resulting from propagating nuclei in the single core ionized state for a time interval $\tau_{1}$. Similarly, $s_{2}\left(\tau_{1}, \tau_{2}\right)$ denotes the instantaneous double core Auger spectrum resulting after a time interval $\tau_{1}$ of nuclear dynamics in the single core ionized state and subsequent nuclear dynamics in the double core ionized state for an interval $\tau_{2}$. The instantaneous single core Auger spectra $s_{1}\left(\tau_{1}\right)$ were weighted here with the joint probability $p_{1}\left(t, t_{1}\right) d t_{1}$ of finding the molecule at time $t$ in the single core ionized state after ionization at time $t_{1}$. Similarly the double core Auger spectra $s_{2}\left(\tau_{1}, \tau_{2}\right)$ were weighted with the joint probability $p_{2}\left(t, t_{1}, t_{2}\right) d t_{1} d t_{2}$ of finding the molecule in double core ionized state given that the first and second ionizations have occurred at times $t_{1}$ and $t_{2}$, respectively.

The joint probability densities $p_{1}\left(t, t_{1}\right)$ and $p_{2}\left(t, t_{1}, t_{2}\right)$ are expressed in terms of conditional probabilities $p_{1}\left(t \mid t_{1}\right)$ and $p_{2}\left(t \mid t_{1}, t_{2}\right)$ by

$$
\begin{gathered}
p_{1}\left(t, t_{1}\right)=p_{1}\left(t \mid t_{1}\right) p_{0}\left(t_{1}\right) \sigma_{0 \rightarrow 1} F\left(t_{1}\right), \\
p_{2}\left(t, t_{1}, t_{2}\right)=p_{2}\left(t \mid t_{1}, t_{2}\right) p_{1}\left(t_{2}, t_{1}\right) \sigma_{1 \rightarrow 2} F\left(t_{2}\right),
\end{gathered}
$$

where $p_{0}(t)$ is the probability of the neutral electronic state at time $t, F(t)$ is the photon flux, and $\sigma_{i \rightarrow j}$ are the partial 


\section{Results}

photoionization cross sections. Here the indices 0,1 , and 2 denote the neutral, single core ionized, and double core ionized states, respectively. The conditional probabilities were obtained from the numerical solution of the master equations

$$
\begin{gathered}
\frac{d p_{0}(t)}{d t}=-p_{0}(t) \sigma_{0} F(t) ; \quad p_{0}(t \rightarrow-\infty)=1, \\
\frac{d p_{1}\left(t \mid t_{1}\right)}{d t}=-p_{1}\left(t \mid t_{1}\right)\left(\Gamma_{1}^{\text {Auger }}+\sigma_{1} F(t)\right) ; \quad p_{1}\left(t_{1} \mid t_{1}\right)=1,
\end{gathered}
$$

$$
\frac{d p_{2}\left(t \mid t_{1}, t_{2}\right)}{d t}=-p_{2}\left(t, \mid t_{1}, t_{2}\right) \Gamma_{2}^{\text {Auger }} ; \quad p_{2}\left(t_{2}, \mid t_{1}, t_{2}\right)=1 .
$$

Here we neglected any geometry (i.e., time) dependence of the total Auger decay rates $\Gamma_{1}^{\text {Auger }}$ and $\Gamma_{2}^{\text {Auger }}$ as well as of the respective photoionization cross sections $\sigma_{i \rightarrow j}$, because they all were found to vary by less than $2 \%$ for all obtained geometries. To gain more insight in the ionization process, also the total populations of the single and double core ionized state at time $t$ were considered, given by

$$
\begin{gathered}
N_{1}(t)=\int_{-\infty}^{t} d t_{1} p_{1}\left(t, t_{1}\right) \\
N_{2}(t)=\int_{-\infty}^{t} d t_{2} \int_{-\infty}^{t_{2}} d t_{1} p_{2}\left(t, t_{1}, t_{2}\right) .
\end{gathered}
$$

\section{H. Illumination conditions}

We assumed a Gaussian x-ray pulse with 10 fs FWHM. Soft x-ray photon beams at photon energies of $1 \mathrm{keV}$ were considered, with peak intensities of 10 photons $/ \mathrm{fs} \AA^{2} \hat{\simeq} 1.6$ $\times 10^{16} \mathrm{~W} / \mathrm{cm}^{2}, 100$ photons $/ \mathrm{fs} \AA^{2} \hat{\simeq} 1.6 \times 10^{17} \mathrm{~W} / \mathrm{cm}^{2}$, and 1000 photons $/ \mathrm{fs} \AA^{2} \hat{\simeq} 1.6 \times 10^{18} \mathrm{~W} / \mathrm{cm}^{2}$, respectively. These parameters agree with the regime offered by the Atomic Molecular and Optical science instrument at the LCLS (Ref. 46) and provide a high ionization rate such that a considerable amount of double core ionizations is reached.

For visible light one might argue that such high fluxes give rise to instantaneous multi-photon ionization or tunne ionization events. These effects are relevant if the ponderomotive energy $U_{p}=\frac{8 \pi}{137} \frac{I}{4 \omega^{2}}$ exceeds the ionization energy. However, for the x-ray pulses considered here, $U_{p} \leq 0.14 \mathrm{eV}$, which is far below the ionization energy. Despite the high intensities, these effects can therefore assumed to be negligible, and thus the perturbative approach of sequential photoionization is justified.

\section{RESULTS AND DISCUSSION}

\section{A. Photoionization cross sections}

We first tested our approach by comparing calculated total photoionization cross sections for neon with values from McMasters compilation of $\mathrm{x}$-ray cross sections ${ }^{47}$ for selected
TABLE I. Calculated total photoionization cross sections for atomic neon compared to values from Ref. 47

\begin{tabular}{lcc}
\hline \hline $\begin{array}{l}\text { Photon } \\
\text { energy } \\
(\mathrm{keV})\end{array}$ & $\begin{array}{c}\text { This work } \\
\text { in } 10^{-3} \\
\text { a.u. }\end{array}$ & $\begin{array}{c}\text { From McMaster } \text { et al. }{ }^{47} \\
\text { in } 10^{-3} \\
\text { a.u. }\end{array}$ \\
\hline 1 & 9.63 & 9.10 \\
2 & 1.53 & 1.51 \\
10 & 0.0137 & 0.0137 \\
\hline \hline
\end{tabular}

photon energies. As shown in Table I, our calculated ionization cross sections agree well with the tabulated values. Table II lists the obtained total and partial cross sections of water for a photon energy of $\omega=1 \mathrm{keV}$. For water, the ratio of the partial cross sections to the single core ionized state to the total cross section $\sigma_{0 \rightarrow 1} / \sigma_{0}$ is about $80 \%$. The remaining $20 \%$ mostly involve core ionization with additional shake-up transitions of valence electrons. A similar ratio is seen for the second ionization step.

\section{B. Auger decay rates}

To also validate Auger decay rate calculations, we compared in Table III calculations for neon with calculation from Kolorenč and Averbukh, ${ }^{48}$ Bhalla et al. ${ }^{49}$ Yarzhemsky and Sgamellotti ${ }^{50}$ (single core), Kelly ${ }^{51}$ (single core), Pelicon et al. ${ }^{52}$ (double core), and $\mathrm{Chen}^{53}$ (double core). The values reported in these studies vary by about $10 \%$ for the single core and by up to $20 \%$ for the double core Auger transition. As can be seen in Table III, our values fall within these ranges.

Table IV compares the calculated single core Auger transition rates for water with previous calculations of absolute ${ }^{15}$ and relative values. ${ }^{26}$ We have adjusted the calculated energies to the experimental spectrum (see Fig. 5) by subtracting an overall offset of $1.1 \mathrm{eV}$. This offset may result from neglecting relativistic effects in our calculation, truncation of the CI space, or incomplete basis sets.

As can be seen, the relative rates (normalized to the dominant $1 b_{1}^{-2} S$ peak) compare well in the higher energy regime, where final states consist of two outer valence holes. In the lower energy range, somewhat larger deviations are seen, which can be explained by the stronger influence of shake-up contributions. Notably, our values tend to be larger than the Auger decay rates obtained by Stieltjes imaging calculations by Carravetta and Ågren ${ }^{15}$ and Kolorenč and Averbukh, ${ }^{48}$ with a total transition rate of $6.0 \times 10^{-3}$ a.u. compared to

TABLE II. Calculated total and partial photoionization cross sections for water at $1 \mathrm{keV}$. The indices 0,1 , and 2 denote the neutral, single core ionized, and double core ionized state, respectively.

\begin{tabular}{lc}
\hline \hline \multirow{2}{*}{ Transition } & Cross section in $10^{-3}$ \\
\hline$\sigma_{0}$ & a.u. \\
$\sigma_{0 \rightarrow 1}$ & 3.84 \\
$\sigma_{1}$ & 3.08 \\
$\sigma_{1 \rightarrow 2}$ & 2.62 \\
\hline \hline
\end{tabular}




\section{Results}

TABLE III. Comparison of calculated Auger decay rates for singly and doubly core ionized neon in $10^{-3}$ a.u

\begin{tabular}{|c|c|c|c|c|}
\hline & Total single col & e Auger decay rate i & $10^{-3}$ a.u & \\
\hline This work & $\begin{array}{c}\text { Kolorenč } \\
\text { and Averbukh }\end{array}$ & $\begin{array}{c}\text { Yarzhemsky } \\
\text { and Sgamellotti }\end{array}$ & Kelly ${ }^{51}$ & Bhalla et al..$^{49}$ \\
\hline 9.9 & 9.2 & 8.9 & 8.1 & 8.8 \\
\hline & $\begin{array}{l}\text { Total double co } \\
\text { Kolorenč }\end{array}$ & Auger decay rate i & $10^{-3}$ a.u & \\
\hline This work & and Averbukh ${ }^{48}$ & Pelicon et al. $^{52}$ & Chen $^{53}$ & Bhalla et al..$^{49}$ \\
\hline 26.1 & 18.6 & 22.9 & 29.5 & 26.0 \\
\hline
\end{tabular}

$5.5 \times 10^{-3}$ a.u. (Ref. 15 ) and $5.4 \times 10^{-3}$ a.u. (Ref. 48 ). However, our value for the total Auger decay rate is similar to the single core hole decay rate measured by Sankari et al.,${ }^{54} 5.8$ $\pm 0.2 \times 10^{-3}$ a.u.

Table V shows transition rates obtained for the double core Auger spectrum of water. As for the single core Auger transitions, energies have been shifted by $1.1 \mathrm{eV}$. We note, however, that relativistic contributions not taken into account in our calculations may contribute about $\simeq 1-2 \mathrm{eV}$ more in the double than in the single core hole case. ${ }^{55}$ The obtained total double core decay rate $\left(18.2 \times 10^{-3}\right.$ a.u. $)$ is about three times larger than the single core decay rate. It is also significantly larger than the value reported by Kolorenč and Averbukh ${ }^{48}$ who used Stieltjes imaging method $\left(11.4 \times 10^{-3}\right.$ a.u. $)$. These authors $^{48}$ estimated their value to be $20 \%$ too low due to insufficient inclusion of initial state orbital relaxation effects. This estimation was based on the discrepancy between thei results and other calculations for atomic neon (see Table III) Whereas Kolorenč and Averbukh ${ }^{48}$ used neutral state opti-
TABLE V. Total $\Gamma_{2}^{\text {Auger }}$ and partial $\Gamma_{2 \rightarrow f}^{\text {Auger }}$ Auger transition rates of water (double core) for the main transition channels (MP2 optimized equilibrium geometry).

\begin{tabular}{lcc}
\hline \hline Channel & Energy in eV & $\Gamma_{\text {ini } \rightarrow \text { fin }}^{\text {Auger }} 10^{-4}$ a.u \\
\hline $1 \mathrm{~b}_{1}^{-2}$ & 556.13 & 24.70 \\
$3 \mathrm{a}_{1}^{-1} 1 \mathrm{~b}_{1}^{-1}$ & 555.31 & 23.89 \\
$1 \mathrm{~b}_{2}^{-1} 1 \mathrm{~b}_{1}^{-1}$ & 552.81 & 18.18 \\
$3 \mathrm{a}_{1}^{-2}$ & 552.35 & 19.11 \\
$3 \mathrm{a}_{1}^{-1} 1 \mathrm{~b}_{2}^{-1}$ & 551.27 & 13.91 \\
$1 \mathrm{~b}_{2}^{-2}$ & 546.79 & 15.58 \\
$2 \mathrm{a}_{1}^{-1} 1 \mathrm{~b}_{1}^{-1}$ & 532.02 & 13.42 \\
$2 \mathrm{a}_{1}^{-1} 3 \mathrm{a}_{1}^{-1}$ & 531.53 & 13.78 \\
$2 \mathrm{a}_{1}^{-1} 1 \mathrm{~b}_{2}^{-1}$ & 527.35 & 9.37 \\
$2 \mathrm{a}_{1}^{-2}$ & 513.27 & 7.65 \\
$\Gamma_{2}^{\text {Auger }}$ & & 182.3 \\
\hline \hline
\end{tabular}

mized orbitals and cover orbital relaxation effects in initial and final states with the ADC(2)x (Ref. 56) method, our calculation is based on initial state optimized orbitals and incorporates final state orbital relaxation by configurational interaction. We therefore assume that our calculation does not suffer from these problems.

\section{Population}

Figure 3 shows the populations of the neutral, single, and double core ionized states obtained from Eqs. (41) and (42) for different beam intensities. The decrease rate of the neutral population increases with pulse intensity, whereas the transition rate to single and double core ionized populations

\begin{tabular}{|c|c|c|c|c|c|c|}
\hline \multirow[b]{2}{*}{ Channel } & \multirow{2}{*}{$\frac{\text { Energy in } \mathrm{eV}}{\text { This work }}$} & \multicolumn{2}{|c|}{$\Gamma_{\mathrm{ini} \rightarrow \text { fin }}^{\text {Auger }}$ in $10^{-4}$ a.u. } & \multicolumn{3}{|c|}{ Relative $\Gamma_{\text {ini } \rightarrow \text { fin }}^{\text {Auger }}$} \\
\hline & & This work & From Ref. 15 & This work & From Ref. 15 & From Ref. 26 \\
\hline $3 \mathrm{a}_{1}^{-1} 1 \mathrm{~b}_{1}^{-1} \mathrm{~T}$ & 500.67 & 0.26 & 0.11 & 3 & 2 & 2 \\
\hline $1 b_{1}^{-2} s$ & 499.39 & 8.25 & 5.79 & 100 & 100 & 100 \\
\hline $3 a_{1}^{-1} 1 b_{2}^{-1} S$ & 497.98 & 7.59 & 5.57 & 92 & 96 & 99 \\
\hline $1 b_{2}^{-1} 1 b_{1}^{-1} \mathrm{~T}$ & 496.60 & 0.00 & 0.00 & 0 & 0 & 0 \\
\hline $1 b_{2}^{-1} 1 b_{1}^{-1} S$ & 494.68 & 6.57 & 5.33 & 80 & 92 & 74 \\
\hline $3 a_{1}^{-2} S$ & 494.64 & 5.74 & 3.93 & 70 & 68 & 71 \\
\hline $3 \mathrm{a}_{1}^{-1} 1 \mathrm{~b}_{2}^{-1} \mathrm{~T}$ & 494.63 & 0.20 & 0.09 & 2 & 2 & 1 \\
\hline $3 a_{1}^{-1} 1 b_{2}^{-1} S$ & 492.36 & 5.62 & 5.57 & 68 & 96 & 58 \\
\hline $1 b_{2}^{-2} \mathrm{~S}$ & 487.45 & 4.55 & 3.45 & 55 & 60 & 34 \\
\hline $2 \mathrm{a}_{1}^{-1} 1 \mathrm{~b}_{1}^{-1} \mathrm{~T}$ & 482.30 & 2.03 & 1.19 & 25 & 21 & 14 \\
\hline $2 \mathrm{a}_{1}^{-1} 3 \mathrm{a}_{1}^{-1} \mathrm{~T}$ & 480.58 & 1.78 & 1.66 & 22 & 29 & 11 \\
\hline $2 \mathrm{a}_{1}^{-1} 1 \mathrm{~b}_{2}^{-1} \mathrm{~T}$ & 476.82 & 1.02 & 1.19 & 12 & 21 & 8 \\
\hline $2 a_{1}^{-1} 1 b_{1}^{-1} S$ & 475.76 & 3.19 & 3.35 & 39 & 58 & 55 \\
\hline $2 a_{1}^{-1} 3 a_{1}^{-1} S$ & 473.27 & 3.86 & 3.74 & 47 & 65 & 48 \\
\hline $2 a_{1}^{-1} 1 b_{2}^{-1} S$ & 468.75 & 2.18 & 2.58 & 26 & 45 & 32 \\
\hline $2 \mathrm{a}_{1}^{-2} \mathrm{~S}$ & 457.19 & 1.51 & 3.54 & 18 & 61 & 48 \\
\hline$\Gamma_{1}^{\text {Auger }}$ & & 60.01 & 55.20 & & & \\
\hline
\end{tabular}




\section{Results}

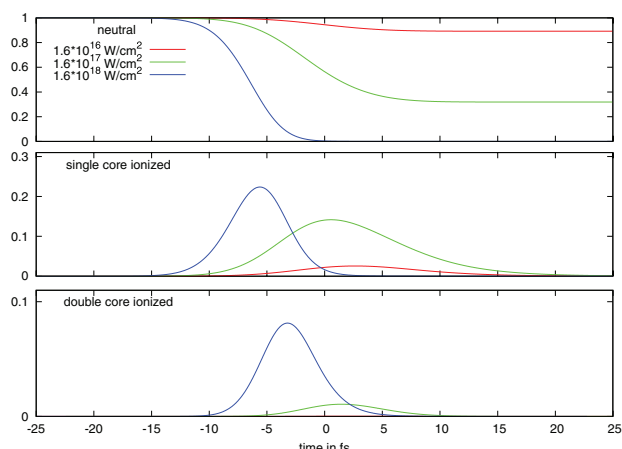

FIG. 3. Integrated population of the neutral $N_{0}(t)$, single core ionized $N_{1}(t)$ and double core ionized states $N_{2}(t)$. A Gaussian shaped x-ray pulse centere at time $t=0 \mathrm{fs}$, width of $10 \mathrm{fs}$ FWHM and a photon energy of $1 \mathrm{keV}$ wa assumed.

increase, such that their peak positions are shifted to earlier times.

As can be seen in Table VI, the probability for double core ionization is about 100 times smaller than for single core ionization at $1.6 \times 10^{16} \mathrm{~W} / \mathrm{cm}^{2}$, and reaches a ratio of $0.465 \mathrm{at}$ $1.6 \times 10^{18} \mathrm{~W} / \mathrm{cm}^{2}$. At $1.6 \times 10^{18} \mathrm{~W} / \mathrm{cm}^{2}$ the first ionization step is saturated, and the probability of first core ionization agrees with the ratio $\sigma_{0 \rightarrow 1} / \sigma_{0}=0.802$. Note that the missing $20 \%$ from shake-up contributions and valence ionization are not considered here and thus are missing in our simulations. After Auger decay the molecule may undergo further core ionizations, as has been observed for Neon. ${ }^{10}$ Note that these further ionization steps, which involve a large number of different channels, are not included in our simulation.

\section{Single and double core ionized Auger spectra}

Figure 4 illustrates trajectories starting from zero velocities and equilibrium geometry by snapshots of the electron densities in the molecular plane, calculated from the CI wavefunctions. The evolution of the $\mathrm{OH}$ bond length is shown in the upper left panel, that of the $\mathrm{HOH}$ bond angle in the supplementary material. ${ }^{40}$ As can be seen, in the single core ionized state the nuclei motion is mainly a bending motion, whereas in the double core ionized state protons are rapidly expelled from the molecule within a few femtoseconds. During that process, and with further ionization, the electron density becomes increasingly isotropic.

TABLE VI. Total probability of single and double core ionization for different flux intensities.

Intensity $\left(\mathrm{W} / \mathrm{cm}^{2}\right) \quad$ 1st core ionization 2 nd core ionization Ratio $2 \mathrm{nd} / 1 \mathrm{~s}$

\begin{tabular}{llll}
\hline $1.6 \times 10^{16}$ & 0.087 & 0.001 & 0.01 \\
$1.6 \times 10^{17}$ & 0.546 & 0.069 & 0.13 \\
$1.6 \times 10^{18}$ & 0.802 & 0.373 & 0.465 \\
\hline
\end{tabular}
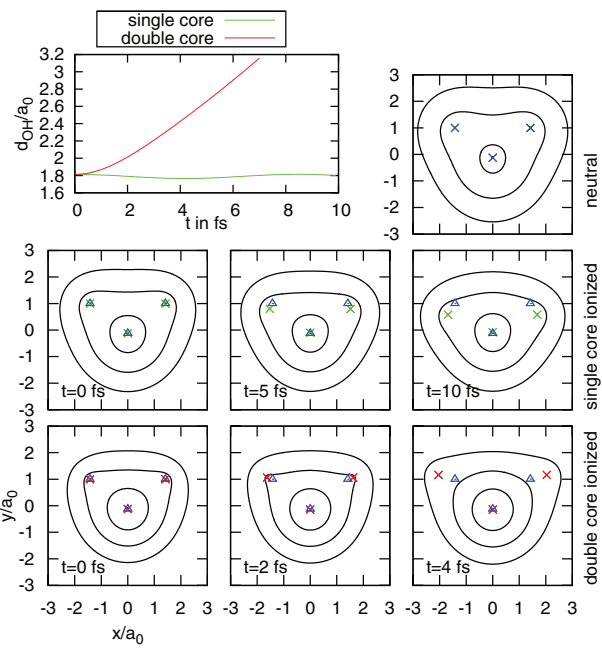

FIG. 4. Dynamics of a water molecule after single (middle row) and double (bottom row) core ionization. The upper left plot shows the evolution of the OH-bond length $d_{\mathrm{OH}}$ for the single and double core ionized state. The other plots show cuts through the electron density in the molecular plane (contour lines) at three selected times for the neutral, single core ionized, and double core ionized state. Crosses denote the positions of the nuclei; triangles mark core neres starting from equilibrium geometry with zero initial velocities.

Figure 5 compares the calculated single core spectrum at peak intensity $1.6 \times 10^{16} \mathrm{~W} / \mathrm{cm}^{2}$ with the spectrum measured by Moddeman et al. ${ }^{45}$ Also shown for comparison are the calculated spectra obtained with frozen nuclei. As can be seen, the calculated spectrum captures most of the features of the experimental spectrum very well. Only small deviations to the spectrum with frozen nuclei are observed. Obviously, for single core ionization the influence of the nuclear motion

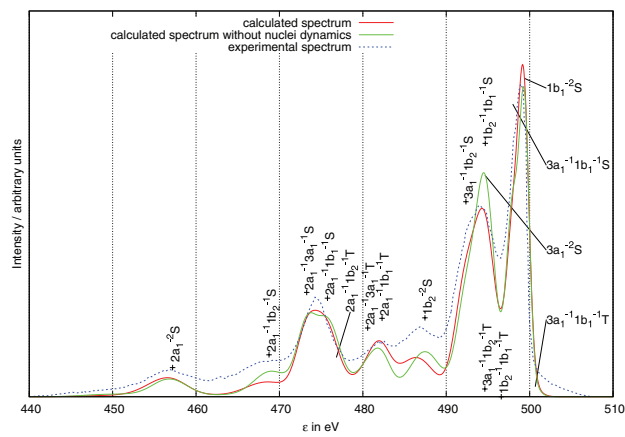

FIG. 5. Single core Auger spectrum. Comparison of experimental spectrum ${ }^{45}$ (dashed line) and calculated spectrum for peak intensity $I=1.6$ $\times 10^{16} \mathrm{~W} / \mathrm{cm}^{2}$ with (red line) and without (green line) nuclei dynamics. The position of the peaks were labeled according to their dominant hole configurations. 


\section{Results}
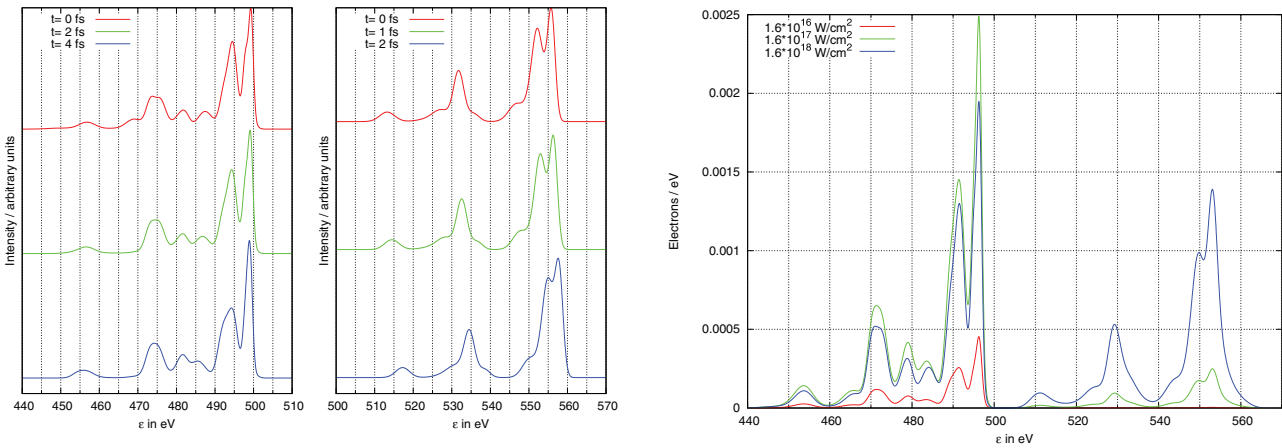

FIG. 6. (Left) Instantaneous single core Auger spectra after $0 \mathrm{fs}, 2 \mathrm{fs}$, an $4 \mathrm{fs}$ of the single core ionized state. (Right) Instantaneous double core Auger spectra after $0 \mathrm{fs}, 1 \mathrm{fs}$, and $2 \mathrm{fs}$ of the double core ionized state. For the double core Auger spectrum, nuclear motion was calculated only for the double core ionized state. The fast dissociation in the double core ionized state is reflected by the fast shift of the Auger spectrum to higher energies.

in the Auger spectrum is rather small, which can also be seen in Fig. 6 (left), where a set of instantaneous Auger spectra of selected snapshots of the single core ionized state trajectories are shown. Indeed, for different times these Auger spectra are very similar, with the notable exception at about 485 $495 \mathrm{eV}$, where final states are associated with vacancies in outer valence orbitals $1 b_{2}$ and $3 a_{1}$, which are mostly af fected by the hydrogen bending movement and thus sensitive to small geometry changes. The valence orbital $1 b_{1}$ is only weakly affected by geometry changes as its nodal plane is identical with the molecular plane.

Figure 7 shows the calculated double core spectrum at peak intensity $1.6 \times 10^{18} \mathrm{~W} / \mathrm{cm}^{2}$ and the calculated spectrum with frozen nuclei. Here the nuclear dynamics causes a long tail to higher energies for each peak in the spectrum. The instantaneous spectra at different time steps (Fig. 6, right) confirm that the spectra indeed shift to higher energies as the

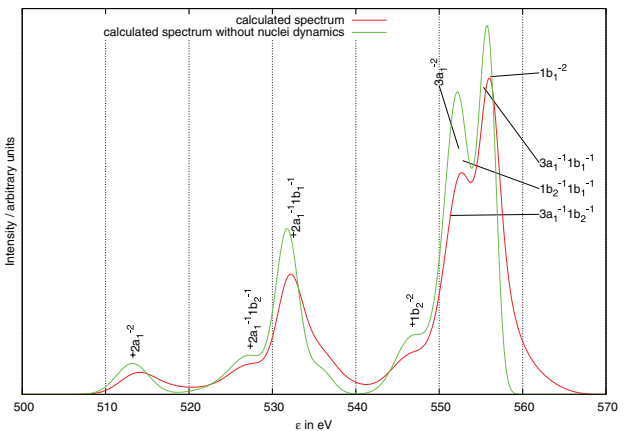

FIG. 7. Double core Auger spectrum. Calculated spectrum for peak intensity $I=1.6 \times 10^{18} \mathrm{~W} / \mathrm{cm}^{2}$ with (red line) and without (green line) nuclei dynamics. The positions of the peaks are labeled according to their dominant hole configurations.

FIG. 8. Single and double core Auger spectrum for different peak intensities. At peak intensity $1.6 \times 10^{18} \mathrm{~W} / \mathrm{cm}^{2}$ the two parts of the Auger spectrum are at comparable intensity.

double core ionized state evolves. This is a result of the strong dissociative motion in the double core ionized state (see Fig. 4, bottom). As both protons are repelled, positive charge is removed from the molecule. As a result, the subsequent Auger recombination involves larger energy differences.

The combined single and double core Auger spectra, composed according to Eqs. (34) and (35), are shown in Fig. 8 for the same flux parameters as in Fig. 3. For 1.6 $\times 10^{16} \mathrm{~W} / \mathrm{cm}^{2}$ the single core Auger spectrum clearly dominates, while at $1.6 \times 10^{17} \mathrm{~W} / \mathrm{cm}^{2}$ the double core spectrum has already significant contribution. At peak intensity 1.6 $\times 10^{18} \mathrm{~W} / \mathrm{cm}^{2}$, the double core and single core spectra reach the same intensity. Here, about $50 \%$ of the population is double core ionized, such that the single core Auger spectrum becomes even smaller than for $1.6 \times 10^{17} \mathrm{~W} / \mathrm{cm}^{2}$.

\section{CONCLUSION}

We have developed a procedure for calculating $a b$ initio transition rates for photoionization and molecular Auger decay, which was validated against previous calculations and experimental data for neon. Our test calculations demonstrates that the single center expansion method in combination with LCAO for the bound MOs provides reliable cross sections and transition rates for water. It was demonstrated that for describing ionization dynamics the second core ionization process must be considered for intensities above $10^{17} \mathrm{~W} / \mathrm{cm}^{2}$.

Auger spectra were computed for a single and double core ionized water molecule. For these calculations, the nuclear dynamics during the core hole lifetime were described by an MD approach based on the core ionized UHF/RHF wavefunction. The obtained total Auger decay rates as well as the spectra agree well with previous experimental data. Strikingly, the Auger decay rate of double core ionized water turned out to be three times larger than that of single core ionized water. Only small effects of the nuclear motion on the single core Auger spectrum were seen. In contrast, for the double core ionized water molecule fast dissociation dynamics is seen, which 


\section{Results}

strongly affects the respective Auger spectra. A particular signature of nuclear motion, which should be seen in future FEL experiments, are marked tails at the higher energy side of most peaks. This signature, therefore, might provide an independent probe for detecting fast nuclear motion on femtosecond time scales. Future work should address possible vibrational interference effects for the nuclear motion, which in our incoherent accumulation of spectra have been neglected. Although only small interference effects are expected for the single core hole Auger spectrum of the water monomer, more pronounced fingerprints might be visible in the double core hole Auger spectrum, and in particular in the spectra of the water dimer as has been demonstrated for the $\mathrm{X}$-ray emission spectrum. ${ }^{57,58}$

\section{ACKNOWLEDGMENTS}

This work has been supported by the DFG, Grant No. SFB 755

${ }^{1}$ R. Neutze, R. Wouts, D. van der Spoel, E. Weckert, and J. Hajdu, "Potential for biomolecular imaging with femtosecond X-ray pulses," Nature (London) 406, 752 (2000)

${ }^{2}$ K. J. Gaffney and H. N. Chapman, "Imaging atomic structure and dynamics with ultrafast X-ray scattering," Science 316, 1444 (2007).

with ultrafast X-ray scattering," Science 316, 1444 (2007). ambropoulos, and A. Mihelič, "Theory of multiphoton multielectron ionization of xenon under strong $93-\mathrm{eV}$ radiation,” Phys. Rev. tt. 102, $33002(2009$

${ }^{4} \mathrm{~N}$. Rohringer and R. Santra, "X-ray nonlinear optical processes using self-amplified spontaneous emission free-electron laser," Phys. Rev. A 76, 033416 (2007)

${ }^{5}$ S. Son, L. Young, and R. Santra, "Impact of hollow-atom formation on coherent x-ray scattering at high intensity,” Phys. Rev. A 83, 033402 (2011)

${ }^{6}$ R. Santra, N. V. Kryzhevoi, and L. S. Cederbaum, "X-ray two-photon photoelectron spectroscopy: a theoretical study of inner-shell spectra of the o ganic para-aminophenol molecule," Phys. Rev. Lett. 103, 013002 (2009). ${ }^{7}$ M. Tashiro, M. Ehara, H. Fukuzawa, K. Ueda, C. Buth, N. V. Kryzhevoi, and L. S. Cederbaum, "Molecular double core hole electron spectroscopy for chemical analysis," J. Chem. Phys. 132, 184302 (2010).

${ }^{8}$ L. S. Cederbaum, F. Tarantelli, A. Sgamellotti, and J. Schirmer, "On double vacancies in the core," J. Chem. Phys. 85, 6513 (1986).

${ }^{9}$ J. H. D. Eland, M. Tashiro, P. Linusson, M. Ehara, K. Ueda and R. Feifel, "Double core hole creation and subsequent auger decay in nh. Feife, molecules," Phys. Rev. Lett. 105, 213005 (2010).

${ }^{10}$ L Youles, 'P. P. Kev. Lett. 105, 213005 (201). L. Young, E. P. Kanter, B. Kraessig, Y. Li, A. M. March, S. T. Prat, R. San, C. A. Roedig, N. Benah, L. Fang, M. Ho , P. H. Bucksbaum, J.P. Cryan, C. Ghire, J. M. Glownia, D. A. Reis, J. D. Bozek, C. Bosted, and M. Messerschmidt, "Femtosecond electronic response of atoms to ultraintense X-rays," Nature (London) 466, 56 (2010).

${ }^{11}$ L. Fang, M. Hoener, O. Gessner, F. Tarantelli, S. T. Pratt, O. Kornilov, C. Buth, M. Guehr, E. P. Kanter, C. Bostedt, J. D. Bozek, P. H. Bucksbaum, M. Chen, R. Coffee, J. Cryan, M. Glownia, E. Kukk, S. R. Leone, and N. Berrah, "Double core-hole production in N-2: beating the Auger clock," Phys. Rev. Lett. 105, 083005 (2010).

${ }^{12}$ C. Gnodtke, U. Saalmann, and J. M. Rost, "Ionization and charge migration through strong internal fields in clusters exposed to intense x-ray pulses," Phys. Rev. A 79, 041201 (2009).

${ }^{13} \mathrm{Z}$. Jurek and G. Faigel, "The effect of tamper layer on the explosion dynamics of atom clusters," Eur. Phys. J. D 50, 35 (2008).

${ }^{14} \mathrm{Z}$. Jurek and G. Faigel, "The effect of inhomogenities on single-molecule imaging by hard XFEL pulses," EPL 86, 68003 (2009).

${ }^{15} \mathrm{~V}$. Carravetta and $\mathrm{H}$. ̊ngen "Stieltjes imaging method for molecular auger transition rates - application to the auger spectrum of water," Phys. Rev. A 35, 1022 (1987).

${ }^{16}$ B. Schimmelpfennig, B. Nestmann, and S. Peyerimhoff, "Ab initio calculation of transition rates for autoionization: the Auger spectra of HF and lation of transition rates for autoionization: the Auger spect
$F^{-}$," J. Electron Spectrosc. Relat. Phenom. 74, 173 (1995).

${ }^{17} \mathrm{R}$. Colle and S. Simonucci, "Method for calculating Auger decay-rates in ${ }^{17}$ R. Colle and S. Simonucci, "Method for cal
molecules," Phys. Rev. A 39, 6247 (1989).
${ }^{18}$ E. Chelkowska and F. Larkins, "Auger-Spectroscopy for molecules-tables of matrix-elements for transition-rate calculations corresponding to an type, p-type, or d-type initial hole," At. Data Nucl. Data Tables 49, 12 (1991)

${ }^{19}$ M. Mitani, O. Takahashi, K. Saito, and S. Iwata, "Theoretical molecular Auger spectra with electron population analysis," J. Electron Spectrosc. Relat. Phenom. 128, 103 (2003).

${ }^{20} \mathrm{M}$. Tashiro, K. Ueda, and M. Ehara, "Auger decay of molecular double core-hole state," J. Chem. Phys. 135, 154307 (2011).

${ }^{21}$ P. Demekhin, A. Ehresmann, and V. Sukhorukov, "Single center method: a computational tool for ionization and electronic excitation studies of molecules," J. Chem. Phys. 134, 024113 (2011).

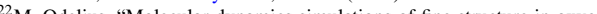
Kedre -edge x-ray emis

${ }^{23}$ O. Takahashi, M. Odelius, D. Nordlund, A. Nilsson, H. Bluhm, and L. Pettersson, "Auger decay calculations with core-hole excited-state molecular-dynamics simulations of water," J. Chem. Phys. 124, 064307 (2006)

${ }^{24}$ M. Eroms, O. Vendrell, M. Jungen, H. Meyer, and L. Cederbaum, "Nuclea dynamics during the resonant Auger decay of water molecules," J. Chem. Phys. 130, 154307 (2009)

${ }^{25}$ Z. Bao, R. Fink, O. Travnikova, D. Ceolin, S. Svensson, and M. Piancastelli, "Detailed theoretical and experimental description of norma Auger decay in $\mathrm{O}_{2}$," J. Phys. B 41, 125101 (2008)

${ }^{26} \mathrm{H}$. Siegbahn, L. Asplund, and P. Kelfve, "The Auger electron spectrum of water vapour," Chem. Phys. Lett. 35, 330 (1975).

${ }^{27}$ R. Pauncz, Spin Eigenfunctions: Construction and Use (Plenum, New York, 1979).

${ }^{28}$ In cases where the total spin quantum numbers are irrelevant these additional indices are skipped in the following.

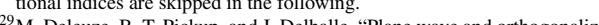
. Delezze, B. T. Pickup, and J. Dehalle, "Plane wave and orthogonalized plane wave many-body green's functioncacul

R. Gaspar, "Uber eine approximation des Hartreefockschen potential durch eine universelle potentialfunktion," Acta Phys. Acad. Sci. Hung. 3, 263 (1954)

${ }^{31}$ P. Demekhin, D. Omelyanenko, B. Lagutin, V. Sukhorukov, L. Werner, A. Ehresmann, K.-H. Schartner, and H. Schmoranzer, "Investigation of photoionization and photodissociation of an oxygen molecule by the method of coupled differential equations," Opt. Spectrosc. 102, 318 (2007). ${ }^{32} \mathrm{~J}$. Sakurai and S. F. Tuan, Modern Quantum Mechanic (Benjamin/Cummings, 1985), Vol. 1.

${ }^{33} \mathrm{R}$. Manne and H. Ågren, "Auger transition amplitudes from general manyelectron wavefunctions," Chem. Phys. 93, 201 (1985).

${ }^{34} \mathrm{~T}$. H. Dunning, "Gaussian basis sets for use in correlated molecular calculations. I. The atoms boron through neon and hydrogen," J. Chem. Phys. 90, 1007 (1989)

${ }^{35}$ T. D. Crawford, C. D. Sherrill, E. F. Valeev, J. T. Fermann, R. A. King, M. L. Leininger, S. T. Brown, C. L. Janssen, E. T. Seidl, J. P. Kenny, and W. D. Allen, "PSI3: an open-source ab initio electronic structure package," J. Comp. Chem. 28, 1610 (2007)

36. S. Bagus, "Self-consistent-field wave functions for hole states of some Ne-like and Ar-like ions," Phys. Rev. 139, A619 (1965). ${ }^{37}$ N. V. Kryzhevoi, R. Santra, and L. S. Cederbaum, "Inner-shell single an double ionization potentials of aminophenol isomers," J. Chem. Phys. 135, 38 .

${ }^{38} \mathrm{E}$. Whittaker and G. Watson, A Course of Modern Analysis (Cambridge University Press, 1952)

${ }^{39}$ L. Biedenharn, J. Louck, and P. Carruthers, Angular Momentum in Quantum Physics: Theory and Application, Encyclopedia of Mathematics an its Applications Series, Vol. 8 (Addison-Wesley, Reading, 1981).

${ }^{40}$ See supplementary material at http://dx.doi.org/10.1063/1.3700233 for detailed results for initial state energies, the obtained ground state optimize geometry and vibrational mode frequencies, and the $\mathrm{HOH}$ bond angle evolution.

${ }^{41}$ L. Chernysheva, Computation of Atomic Processes: A Handbook for the ATOM Programs, Bd. 1 (Institute of Physics, 1997).

${ }^{42} \mathrm{~J}$. McDougall, "The calculation of the terms of the optical spectrum of an atom with one series electron," Proc. R. Soc. Lond. Ser. A 138, 550 (1932), see http://rspa.royalsocietypublishing.org/content/138/836/ 550.full.pdf+html.

${ }^{43}$ D. Frenkel and B. Smit, Understanding Molecular Simulation (Academic, San Diego, CA, 1996). 


\section{Results}

${ }^{44}$ M. J. Frisch, G. W. Trucks, H. B. Schlegel et al., Gaussian 09, Revision A. 02 , Gaussian, Inc., Wallingford, CT, 2009

${ }^{45}$ W. Moddeman, T. Carlson, M. Krause, B. Pullen, W. Bull, an G. K. Schweitz, "Determination of K-LL auger spectra of $\mathrm{N}_{2}, \mathrm{O}_{2}, \mathrm{CO}$, NO, $\mathrm{H}_{2} \mathrm{O}$, and CO," J. Chem. Phys. 55, 2317 (1971).

${ }^{46}$ SLAC National Accelerator Laboratory, "Atomic, molecular \& optical science," 2012, see http://lcls.slac.stanford.edu/amo/.

${ }^{47}$ W. McMaster, N. del Grande, J. Mallett, and J. Hubbell, "Compilation of X-ray cross sections," Lawrence Radiation Laboratory Report No. UCRL50174, Sec. II, Rev. 1 (1969)

${ }^{48} \mathrm{P}$. Kolorenč and V. Averbukh, "K-shell Auger lifetime variation in dou. Ko ion (2011).

C. P. Bhalla, N. O. Folland, and M. A. Hein, "Theoretical $K$-shell Auger rates, transition energies, and fluorescence yields for multiply ionize neon," Phys. Rev. A 8, 649 (1973).

${ }^{50} \mathrm{~V}$. Yarzhemsky and A. Sgamellotti, "Auger rates of second-row atoms calculated by many-body perturbation theory," J. Electron Spectrosc. Relat. Phenom. 125, 13 (2002).

${ }^{51}$ H. P. Kelly, "K Auger rates calculated for $\mathrm{Ne}^{+}$," Phys. Rev. A. 11, 556 (1975)
${ }^{52}$ P. Pelicon, I. Čadež, M. Žitnik, Ž. Šmit, S. Dolenc, A. Mühleisen, an R. I. Hall, "Formation of the hollow $1 s^{o 1} S$ state of $\mathrm{Ne}^{2+}$ by electron impact: observation by means of an Auger hypersatellite," Phys. Rev. A 62, 022704 (2000)

${ }^{53}$ M. H. Chen, "Auger transition rates and fluorescence yields for the double$K$-hole state," Phys. Rev. A 44, 239 (1991).

${ }^{54}$ R. Sankari, M. Ehara, H. Nakatsuji, Y. Senba, K. Hosokawa, H. Yoshida, A. D. Fanis, Y. Tamenori, S. Aksela, and K. Ueda, "Vibrationally resolved O 1s photoelectron spectrum of water," Chem. Phys. Lett. 380, 647 (2003). 55 J. Niskanen, P. Nom $\mathrm{H}$. Aksela, " . Niskanen, P. Norman, H. Aksela, and H. Agren, "Relativistic contributions to single and double core electron ionization energies of noble gases, J. Chem. Phys. 135, 054310 (2011).

${ }^{56}$ P. Kolorenč, V. Averbukh, K. Gokhberg, and L. S. Cederbaum, "Ab initio calculation of interatomic decay rates of excited doubly ionized states in (2008)

${ }^{57}$ M. P. Ljungberg, A. Nilsson, and L. G. M. Pettersson, "Semiclassical description of nuclear dynamics in x-ray emission of water," Phys. Rev. B 82 245115 (2010)

${ }^{58}$ M. P. Ljungberg, L. G. M. Pettersson, and A. Nilsson, "Vibrational interference effects in $\mathrm{x}$-ray emission of a model water dimer: implications for the interpretation of the liquid spectrum," J. Chem. Phys. 134, 044513 (2011). 


\subsection{Double core hole decay and core hole screening in first row hydrides}

\subsubsection{Introduction}

Inspired by the findings of the previous article, we subsequently investigated the decay rate of single and double $\mathrm{K}$ holes for a series of molecules, the first-row hydrides. The enhancement of the decay rate of double K-holes turned out to be a systematic effect occurring in all studied molecules. We discuss possible reasons for this enhancement and relate it to the molecular core hole screening effect. In essence, these screening effect consists of a valence electron transfer from the outer parts of the molecule towards the nucleus, on which the core hole is located due to the uncompensated nucleus charge. This screening effect can also be determined from simple population analysis, which allows estimating the core hole decay rate from simpler quantum chemical procedures. The resulting article was published in the Journal of Chemical Physics.

\section{Individual contributions of the authors}

I performed the calculations, the analysis of the results, and drafted the manuscript. G. Groenhof and H. Grubmüller helped to develop the relation of the total Auger decay rate to the electron population and supervised the project. They both contributed to the manuscript. 


\title{
3 Results
}

\subsubsection{Article}

Reprinted with permission from The Journal of Chemical Physics, 138, 164304 (2013). Copyright 2013, American Institute of Physics.

THE JOURNAL OF CHEMICAL PHYSICS 138, 164304 (2013)

\section{Core hole screening and decay rates of double core ionized first row hydrides}

\author{
L. Inhester, ${ }^{1, a)}$ G. Groenhof, ${ }^{2}$ and H. Grubmüller ${ }^{1}$ \\ ${ }^{1}$ Max Planck Institute for Biophysical Chemistry, Am Faßberg 11, 37077 Göttingen, Germany \\ ${ }^{2}$ Department of Chemistry and Nanoscience Center, University of Jyväskylä, P.O. Box 35, \\ FI-40014 Jyväskylä, Finland
}

(Received 14 February 2013; accepted 29 March 2013; published online 22 April 2013)

Because of the high intensity, X-ray free electron lasers allow one to create and probe double core ionized states in molecules. The decay of these multiple core ionized states crucially determines the evolution of radiation damage in single molecule diffractive imaging experiments. Here we have studied the Auger decay in hydrides of first row elements after single and double core ionization by quantum mechanical ab initio calculations. In our approach the continuum wave function of the emitted Auger electron is expanded into spherical harmonics on a radial grid. The obtained decay rates of double K-shell vacancies were found to be systematically larger than those for the respective single $\mathrm{K}$-shell vacancies, markedly exceeding the expected factor of two. This enhancement is attributed to the screening effects induced by the core hole. We propose a simple model, which is able to predict core hole decay rates in molecules with low $Z$ elements based on the electron density in the vicinity of the core hole. (c) 2013 AIP Publishing LLC. [http://dx.doi.org/10.1063/1.4801660]

\section{INTRODUCTION}

In ultra short X-ray pulse coherent diffractive imaging experiments with single macromolecules, Auger decay and ionization are the primary processes that lead to radiation damage and, ultimately to Coulomb explosion. ${ }^{1,2}$ At the photon energies required for such experiments, photo ionization of core electrons is the dominant ionization process. For elements with a low atomic number $Z$, core ionization induces Auger decay, during which a valence electron refills the core, while a second valence electron is emitted. Thereby, the sample is charged up in sequences of photo ionization and Auger decay events, which eventually leads to a Coulomb explosion. To determine how this structure disintegration proceeds and whether it is possible to collect sufficient scattered photons of the unperturbed structure, knowledge of molecular Auger decay rates is essential.

At the high intensities required for single molecule diffractive imaging, the ionization rates become comparable to Auger decay rates and therefore electronic states with double vacancies in the $\mathrm{K}$ shell become relevant. Further, these states have a much smaller ionization cross section and thus can effectively suppress the formation of high charge states at short pulse durations - a phenomenon known as "frustrated absorption." ${ }^{3,4}$ For these reasons, the lifetimes of the intermediate single and double $\mathrm{K}$-shell vacant states determine the competition of scattering and destruction of the sample.

The investigation of multiple core hole states has recently received attention also because multiple core hole spectroscopy opens up a way to sensitively probe electronic structure. $^{5-8}$ The Auger process in double core hole states in molecules of low $Z$ elements, such as $\mathrm{N}, \mathrm{C}, \mathrm{O}$ has been

a) linhest@gwdg.de studied in a series of theoretical ${ }^{9-11}$ and experimental ${ }^{10,12}$ studies. However, to our knowledge only few attempts have been made to study the decay rate of doubly K-shell ionized states in molecules or atoms. Because in these states there are twice the number of decay channels available compared to the single core ionized case, one may be tempted to assume that the decay rate is simply twice the single core hole decay rate. Indeed, a recent calculation ${ }^{11}$ of the double core hole decay (KK-KLL) of a series of small molecules seems to support such doubled rate, albeit this calculation may not have covered relevant relaxation effects sufficiently, and thus the authors estimated that the true value might be about $20 \%$ larger. ${ }^{11}$ For atoms, Chen ${ }^{13}$ calculated double K-hole vacancy Auger rates (KK-KLL) for atomic numbers $Z=10 \ldots 36$ and found that for lower $Z$ elements the Auger decay rate of double core shell vacancies was significantly larger than twice the Auger decay rate for the single core shell vacancy case. For the water molecule, we have recently calculated an even larger rate increase by a factor of three. ${ }^{14}$

A possible explanation for this pronounced rate increase over single core hole Auger decay are electronic relaxation effects. In particular, a single or double core hole in an atom or molecule induces relaxation of the surrounding electron density, such that the remaining electrons tend to screen the core hole. It can be anticipated that the valence electron density is deformed more dramatically in case of a double core vacancy, compared to that in the presence of a single core hole.

For atoms the additional contraction of electronic shells in a double core hole vacancy state results in an increased decay rate. ${ }^{13,15}$ In molecules, additionally, the shift of valence electrons from neighboring atoms towards the core hole needs to be considered. ${ }^{16}$ As we will show, this effect further increases the decay rates of double core ionized states. 


\section{Results}

To systematically investigate such effects in smal molecules, we have computed the Auger decay rates of a series of single and double core ionized molecules via $a b$ initio quantum chemical methods and analyzed the respective electron density. We have chosen a sequence of isoelectronic molecules, namely, the neutral first-row hydrides methane $\mathrm{CH}_{4}$, ammonia $\mathrm{NH}_{3}$, water $\mathrm{H}_{2} \mathrm{O}$, and hydrogenfluoride $\mathrm{HF}$, as well as their singly charged protonated states, $\mathrm{CH}_{3}^{+}, \mathrm{OH}^{-}, \mathrm{H}_{3} \mathrm{O}^{+}, \mathrm{NH}_{4}^{+}, \mathrm{NH}_{2}^{-}, \mathrm{F}^{-}$, and $\mathrm{H}_{2} \mathrm{~F}^{+}$. All of these molecules have ten electrons, so that any variation of decay rates can be attributed solely to their different electronic structure.

\section{METHODS}

\section{A. Theory}

As described in detail before ${ }^{14}$ Auger decay rates were calculated using a single center expansion of the electronic wave functions. ${ }^{17}$ The bound part of the molecular states was calculated from Configuration Interaction (CI) calculations, while the continuum part was determined by solving the Schrödinger equation in the potential generated by the left-behind molecular cation. To this end, the molecular wave function was expanded into spherical harmonics on a radia grid. Accordingly, the molecular orbitals and single electron continuum wave function are given by

$$
\phi_{i, \sigma}(\mathbf{r})=\sum_{l m} \frac{P_{l m}^{i}(r)}{r} Y_{l m}(\theta, \phi),
$$

where $Y_{l m}(\theta, \phi)$ are spherical harmonics and $P_{l m}^{i}(r)$ radial parts of the expansion. The exchange interaction of the bound electrons with the continuum electron was approximated here by the $\mathrm{KSG}^{18}$ exchange interaction potentia functional

$$
V_{\mathrm{XC}}[\rho(\mathbf{r})]=-\left(\frac{3}{\pi} \rho(\mathbf{r})\right)^{\frac{1}{3}}
$$

of the bound molecular electron density $\rho(\mathbf{r})$. From the determined continuum solutions, first order transition rates for Auger decay,

$$
\Gamma_{\text {ini } \rightarrow \text { fin }}^{\text {Auger }}=2 \pi\left|\left\langle\psi_{\text {fin }}\left|H-E_{\text {ini }}\right| \psi_{\text {ini }}\right\rangle\right|^{2},
$$

were calculated for all relevant final states, including all available continuum channels. Under the assumption that the fluorescence decay channel can be neglected, these rates were finally summed up to obtain the total decay rate.

\section{B. Computational details}

All molecular geometries used here were obtained by geometry optimization for the electronic ground state using GAUSSIAN $09^{19}$ at the MP2/cc-pVTZ level of theory. ${ }^{20}$ For both, initial and final electronic states, CI calculation were based on molecular orbitals (MOs), which were obtained from a restricted (open shell) Hartree-Fock calculation for the respective initial single or double core ionized electronic states using Dunning's cc-pVTZ basis set. ${ }^{21}$ The
Hartree-Fock calculations and the transformation of two electron integrals were performed using a modified PSI3 quantum package. $^{22}$

For the CI calculation we used a slightly different truncation scheme than that in Ref. 14. In particular we included all singly and doubly excited configurations from the respective reference(s). For the initial state, which is the singly or doubly core ionized state, we used a single reference, with a singly or doubly occupied core shell. For the final states of the Auger decay of a single core hole, we used multiple references with all possible combinations of rearranged two valence holes and refilled core shell. For the final states of the Auger decay of a double core hole, the same references were used but with a single occupied core shell. For all core vacant states, i.e., initial states and final states of the double core hole decay, single and double excited configurations were restricted by requiring the core orbital either singly or doubly occupied. This approach served to avoid any refilling of the core hole due to the pseudo continuum formed by the higher energetic valence orbitals.

We note that our rates for neon and water are slightly smaller (by $\leq 10 \%$ ) than those we have reported previously. ${ }^{14}$ This difference is explained by the smaller CI space used in Ref. 14, in which double excitations were limited up to a certain Hartree-Fock orbital eigenvalue. The CI truncation scheme used in the present work allows a higher flexibility of the wave function and therefore improved the description of the final states based on MOs optimized for the initial core ionized state.

The computational parameters for the single center ex pansion were chosen as in Ref. 14. For the linear molecules $\mathrm{HF}, \mathrm{OH}^{-}$and atoms $\mathrm{Ne}, \mathrm{F}^{-}$, the number of $m$ quantum numbers was reduced by exploiting molecular symmetries.

\section{RESULTS AND DISCUSSION}

To validate our method, we first calculated molecular K-LL Auger decay rates for which calculated ${ }^{11,13,15,16}$ or measured ${ }^{23-25}$ values are available. As can be seen in Table I, the single core (K-LL) Auger decay rates we obtained agree with those obtained by Kolorenč and Averbukh ${ }^{11}$ within $4 \%$. For methane and water decay rates, which have been measured via the linewidth, also agree within $12 \%$ with our

TABLE I. Calculated K-LL Auger decay rates $\Gamma_{K}$ in $10^{-3}$ a.u. $^{\text {a }}$ compared with literature values.

\begin{tabular}{lccccc}
\hline \hline & \multicolumn{3}{c}{ Theory } & & \multicolumn{2}{c}{} \\
\cline { 2 - 4 } & This work & Ref. 16 & Ref. 11 & \multicolumn{2}{c}{ Experiment } \\
\hline $\mathrm{CH}_{4}$ & 3.2 & 3.5 & 3.1 & $3.4-3.5^{\mathrm{b}}$ & $3.1 \pm 0.4^{\mathrm{c}}$ \\
$\mathrm{NH}_{3}$ & 4.3 & 5.2 & 4.5 & & \\
$\mathrm{H}_{2} \mathrm{O}$ & 5.6 & 6.8 & 5.4 & $5.8 \pm 0.2^{\mathrm{d}}$ & \\
$\mathrm{HF}$ & 7.3 & 8.3 & & & \\
\hline \hline
\end{tabular}

1 a.u. $=4.13 \times 10^{16} 1 / s$

${ }^{b}$ From Ref. 23

From Ref. 24

${ }^{\mathrm{d}}$ From Ref. 25 


\section{Results}

TABLE II. Calculated KK-KLL Auger decay rates $\Gamma_{K K}$ in $10^{-3}$ a.u. for neon compared with previous calculation.

\begin{tabular}{lccc}
\hline \hline & This work & Ref. 15 & Ref. 13 \\
\hline$\Gamma_{K}$ & 9.2 & 8.8 & 10.3 \\
$\Gamma_{K K}$ & 25.8 & 26.0 & 29.5 \\
$\Gamma_{K K} /\left(2 \Gamma_{K}\right)$ & 1.4 & 1.5 & 1.4 \\
\hline \hline
\end{tabular}

results. Although the values calculated by Larkins ${ }^{16}$ are systematically larger by about $15 \%$ than ours, the relative rate differences within the considered series of molecules also agree well with our results.

Table II compares the calculated single and double core hole Auger decay rates for neon with those calculated by Bhalla et al. ${ }^{15}$ and Chen. ${ }^{13}$ Again, the values we obtained agree within $13 \%$ to their calculations. Notably, for all double core hole decay calculations of neon, a marked enhancemen $\Gamma_{K K} /\left(2 \Gamma_{K}\right)$ of about $1.4-1.5$ is seen. From this comparisons we conclude that our approach yields sufficiently accurate results to quantitatively analyze the systematic trends discussed further below.

As the main result, we have calculated single (K-LL) and double (KK-KLL) core hole decay rates for the above isoelectronic series of molecules (Table III). As can be seen, with increasing atomic number $Z$ of the central atom, the decay rate increases both for single and for double core ionized states. In contrast, the protonation state of the molecule seems to have only a minor effect on both single and double core hole decay rates. Overall, and in line with the results that have been obtained for atoms, ${ }^{13,15}$ all molecular double core hole decays $\Gamma_{K K}$ per core hole (i.e., divided by two) are markedly faster than that of the single core hole $\Gamma_{K}$.

Remarkably, for double core ionized states, the relative increase of the decay rate with $Z$ appears to be slightly smaller. In fact, the ratio of double to single core hole decay rate per core hole, $\Gamma_{K K} /\left(2 \Gamma_{K}\right)$, decreases monotonically with increasing $Z$, from about $\Gamma_{K K} /\left(2 \Gamma_{K}\right)=1.8$ for methane to about $\Gamma_{K K} /\left(2 \Gamma_{K}\right)=1.4$ for neon. Thus, not only the above systematic rate enhancement resembles the trend observed ${ }^{13}$ for atoms with atomic number $Z=10 \ldots 36$, but also its systematic variation of the rate with $Z$.

We therefore asked whether and how this trend can be explained in terms of electronic structure changes due to the core hole, both as a function of $Z$ as well as the number of hy drogen atoms. Specifically, we will analyze valence electron density shifts from the peripheral hydrogen atoms towards the central atom. To this aim, we will introduce a simplified model that relates the decay rates $\Gamma_{K}$ and $\Gamma_{K K}$ to the valence electron population on the central atom, applying a series of approximation steps.

Neglecting correlation effects, we first assume that the dominant contribution to an individual Auger transition rate results from a single pair of initial and final configuration state functions (CSF), both described by the same set of MOs. The amplitude for the Auger process from a CSF with single $1 s$ core hole to a final CSF with vacancies in the valence MOs $v$ and $v^{\prime}$ with either singlet or triplet spin symmetry is
TABLE III. Auger decay rates in $10^{-3}$ a.u. for single core ionized $\left(\Gamma_{K}\right)$ and double core ionized $\left(\Gamma_{K K}\right)$ molecules.

\begin{tabular}{lcccc}
\hline & $Z$ & $\Gamma_{K}$ & $\frac{\Gamma_{K K}}{2}$ & $\frac{\Gamma_{K K}}{2 \Gamma_{K}}$ \\
\hline $\mathrm{CH}_{4}$ & 6 & 3.2 & 5.8 & 1.8 \\
$\mathrm{CH}_{3}^{-}$ & 6 & 3.0 & 5.4 & 1.8 \\
$\mathrm{NH}_{4}^{+}$ & 7 & 4.3 & 8.1 & 1.9 \\
$\mathrm{NH}_{3}$ & 7 & 4.3 & 7.2 & 1.7 \\
$\mathrm{NH}_{2}^{-}$ & 7 & 4.1 & 6.9 & 1.7 \\
$\mathrm{H}_{3} \mathrm{O}^{+}$ & 8 & 5.6 & 9.0 & 1.6 \\
$\mathrm{H}_{2} \mathrm{O}$ & 8 & 5.6 & 8.8 & 1.6 \\
$\mathrm{OH}^{-}$ & 8 & 5.6 & 8.9 & 1.6 \\
$\mathrm{H}_{2} \mathrm{~F}^{+}$ & 9 & 7.2 & 10.8 & 1.5 \\
$\mathrm{HF}$ & 9 & 7.3 & 10.9 & 1.5 \\
$\mathrm{~F}^{-}$ & 9 & 7.4 & 11.0 & 1.5 \\
$\mathrm{Ne}$ & 10 & 9.2 & 12.9 & 1.4 \\
\hline \hline
\end{tabular}

expressed by ${ }^{26}$

$$
\begin{aligned}
& \frac{M_{v v^{\prime}}}{\sqrt{2 \pi}} \\
& =\left\{\begin{array}{cc}
\langle 1 s k|1 / r| v v\rangle & \text { for } v=v^{\prime} \text {, singlet } \\
\frac{1}{\sqrt{2}}\left(\left\langle 1 s k|1 / r| v v^{\prime}\right\rangle+\left\langle 1 s k|1 / r| v^{\prime} v\right\rangle\right) & \text { for } v \neq v^{\prime} \text {, singlet } \\
\frac{1}{\sqrt{2}}\left(\left\langle 1 s k|1 / r| v v^{\prime}\right\rangle-\left\langle 1 s k|1 / r| v^{\prime} v\right\rangle\right) & \text { for } v \neq v^{\prime} \text {, triplet . }
\end{array}\right.
\end{aligned}
$$

The above two-electron integrals are defined as

$$
\begin{aligned}
\left\langle 1 s k|1 / r| v v^{\prime}\right\rangle:= & \int d^{3} r_{1} \int d^{3} r_{2} \phi_{1 s}\left(\mathbf{r}_{1}\right) \phi_{v}\left(\mathbf{r}_{1}\right) \\
& \times \frac{1}{\left|\mathbf{r}_{1}-\mathbf{r}_{2}\right|} \phi_{k}\left(\mathbf{r}_{2}\right) \phi_{v^{\prime}}\left(\mathbf{r}_{2}\right),
\end{aligned}
$$

where $\phi_{1 s}(\mathbf{r})$ denotes the core orbital, $\phi_{k}(\mathbf{r})$ the continuum wave function; $\phi_{v}(\mathbf{r})$ and $\phi_{v^{\prime}}(\mathbf{r})$ are valence orbitals. The KK KLL Auger decay rate of double $\mathrm{K}$ hole states is given by a similar expression, except that the final states have doublet spin symmetry, resulting in an additional factor of two for the rates.

Due to the strong localization of the hole $\phi_{1 s}$, we further assume that the two-electron integrals in Eq. (4) are dominated by those parts of the valence electron wave function $\phi_{v}(\mathbf{r})$ and $\phi_{v^{\prime}}(\mathbf{r})$ which are close to the core hole $\phi_{1 s},{ }^{27,28}$ whereas the contribution of the remaining part of these orbitals to $M_{v v^{\prime}}$ is assumed to be small.

With the above assumptions, and following Refs. 29-32, we approximate each molecular Auger transition rate by

$$
I_{v v^{\prime}}=\left|M_{v v^{\prime}}\right|^{2} \simeq \sum_{a a^{\prime}} C_{v a}^{2} C_{v a^{\prime}}^{2} A_{a a^{\prime}} .
$$

Here, $A_{a a^{\prime}}$ is an atomic Auger rate for a final state with vacancies in the atomic orbitals $a$ and $a^{\prime}$, with populations $C_{v a}^{2}$ and $C_{v^{\prime} a^{\prime}}^{2}$ in the molecular orbitals $v$ and $v^{\prime}$, respectively. Further, we assume that the atomic Auger rates $A_{a a^{\prime}}$ are independent of $Z$; indeed, calculated Auger radial matrix elements ${ }^{33,34}$ for atoms with $6 \leq Z \leq 10$ vary by less than $6 \%$. Also, the atomic Auger rates $A_{a a^{\prime}}$ for the different decay paths (i.e. two-hole combinations $a, a^{\prime}$ ) are replaced by an averaged rate 


\section{Results}

$\hat{A}$, such that the variation of the molecular decay rate $I_{v, v^{\prime}}$ is determined solely by the changes of the populations $C_{v a}^{2}, C_{v^{\prime} a}^{2}$ of the atomic valence orbitals. ${ }^{28}$

If this scenario holds true, the total decay rate $\Gamma$ of a core shell vacancy should be proportional to the number of possible combinations of creating two holes in the valence electrons that occupy the atomic orbitals of the central atom. To test this idea, we estimated the number of valence electron $n_{\text {val }}$ from the Mulliken population on that atom from the $\mathrm{CI}$ wave function for the single or double core ionized state, respectively. It follows that the rates should approximately obey

$\Gamma=\left\{\begin{array}{cc}\hat{A}^{(\mathrm{K})} \frac{1}{2} n_{\mathrm{val}}^{(K)}\left(n_{\mathrm{val}}^{(K)}-1\right) & \text { for a single core hole, } \\ \hat{A}^{(\mathrm{KK})} n_{\mathrm{val}}^{(K K)}\left(n_{\mathrm{val}}^{(K K)}-1\right) & \text { for a double core hole, }\end{array}\right.$

i.e., the relationship between $\Gamma_{K}$ and $\Gamma_{K K}$ and the number of possible combinations of creating two holes, $n_{\mathrm{val}}\left(n_{\mathrm{val}}-1\right) / 2$, should be linear.

Overall, as can be seen from Fig. 1(a), both decay rates indeed follow this expected trend according to Eq. (7). To test whether the remaining deviations are mainly due to ou particular - and somewhat arbitrary - choice of population analysis, Fig. 1(b) shows the same data, but with the number of valence electrons $n_{\text {val }}$ derived from Löwdin populations ${ }^{35}$ instead. Because only minor changes are seen, the deviations are likely to have a different origin.

To address this issue, we note that, whereas the effect of varying $Z$ seems to be well described for neutral molecules by both approaches, the electron populations seem to be larger for the anions than expected from the respective decay rates, and smaller for the cations. In fact, inspection of the respec-
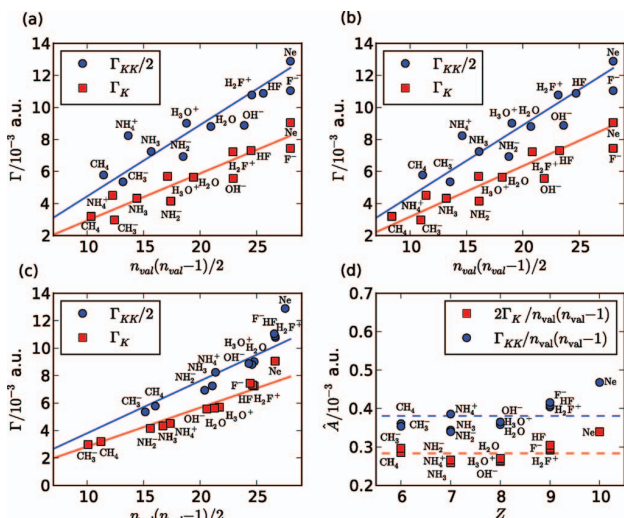

FIG. 1. (a)-(c) Decay rate per core hole as a function of the number of valence electron pairs $n_{\mathrm{v}}\left(n_{\mathrm{v}}-1\right) / 2$ on the atom at which the core hole is located, estimated from the respective core ionized ste wave function is loMulliken and (b) Löwdin population analysis, as well as via (c) the ineg (a) election den (d) Den density within a sphere of radius $r=1.9 a_{0}$ ) around the core hole. (d) Decay rate per core hole and per valence electon pair, where the number

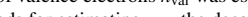
ods for estimating $n_{\mathrm{val}}$ the decay rate $\Gamma_{\mathrm{K}}$ and $\Gamma_{\mathrm{KK}}$ is roughly proportional to $n_{\text {val }}\left(n_{\text {val }}-1\right) / 2$. tive electron density distributions shows only little differences for varying protonation state in the vicinity of the central atom. Apparently, both population analyzes used above markedly exaggerate the changes of the electron density relevant for Auger decay caused by different protonation states.

We therefore asked if, instead, a simple estimate of $n_{\text {val }}$ via the integrated total valence electron density within sphere of a certain radius $r$ around the central atom might better capture the total electron density relevant for the Auger two-electron integrals. We chose a value of $r=1.9 a_{0}$, a value close to the average bond length, for which the integrated electron density yields populations comparable with the Mulliken and Löwdin population. For this choice Eq. (7) predicts all decay rates much better [Figure 1(c)], except for neon. Qualitatively similar results are obtained for $1.5 a_{0}<r$ $<2.5 a_{0}$ (data not shown).

Overall, despite the relatively crude approximations used, Eq. (7) describes the Auger decay rates of the considered series of molecules with varying $Z-$ both for the two wave function based and for the density based population estimates - remarkably well.

Notably, in Figs. 1(a)-1(c), the slope for $\Gamma_{\mathrm{KK}} / 2$, i.e., the averaged atomic decay rate $\hat{A}^{(\mathrm{KK})}$, is about $35 \%$ larger than that for $\Gamma_{\mathrm{K}}$. This trend is quantified in Fig. 1(d), which displays the ratios $\hat{A}^{(\mathrm{K})}=2 \Gamma_{\mathrm{K}} /\left[n_{\mathrm{val}, \mathrm{K}}\left(n_{\mathrm{val}, \mathrm{K}}-1\right)\right]$ and $\hat{A}^{(\mathrm{KK})}$ $=\Gamma_{\mathrm{KK}} /\left[n_{\mathrm{val}, \mathrm{KK}}\left(n_{\mathrm{val}, \mathrm{KK}}-1\right)\right]$ as a function of $Z$. Because a similar value of enhancement has been found for neon (40\%), we attribute this $35 \%$ larger average atomic decay rate $\hat{A}$ to an atomic effect.

Taken together, we have identified and quantified two effects which dominate the systematic differences and trends fo single and double core hole Auger decay rates $\Gamma_{\mathrm{KK}} / 2$ over $\Gamma_{\mathrm{K}}$ for the series of iso-electronic molecules studied here. The rate enhancement for the double core hole over single core hole is, first, due to an increased valence population in the vicinity of the core hole and, second, due to an systematic increase of the Auger decay rate per core hole and valence electron pair. The rate increase with atomic number $Z$ can also be attributed to an increased valence electron population on the atom with the core hole. Generalizing these ideas, we propose that the presented approach is applicable to other molecules in which single and double core holes (single-sited) are located on elements with low atomic number $Z$.

We note that in this work effects of nuclear motion have been neglected. For the water molecule these effects have previously been found to affect the spectrum of the Auger electrons. ${ }^{14}$ However, the total decay rate, which we address here, turned out to be nearly independent of the nuclear dynamics. We therefore assume that this is also the case for the molecules considered here.

\section{SUMMARY AND CONCLUSION}

In summary, we have calculated molecular Auger decay rates for single core hole (K-LL) and double core hole (KKKLL) states for the iso-electronic series of first row hydrides with atomic numbers $Z=6 \ldots 10$ and their singly charged protonated states. We demonstrated that decay of molecular double core hole states is $40 \%$ to $90 \%$ faster than twice the decay 


\section{Results}

of the single core hole. This finding is partially explained by a valence electron shift towards the atom at which the core hole is localized. An unexpectedly simple model based on population analysis enables one to estimate Auger decay rates in molecules. As molecular Auger decay of multiple core holes is a crucial factor for the charging up of molecular samples in coherent diffractive imaging experiments with XFEL, ${ }^{1,2}$ our approach should help to develop efficient descriptions and models of how radiation damage evolves after multiple core ionization also in larger molecules.

\section{ACKNOWLEDGMENTS}

This work has been supported by the Deutsche Forschungsgemeinschaft (DFG), Grant No. SFB 755.

${ }^{1}$ K. J. Gaffney and H. N. Chapman, "Imaging atomic structure and dynamics with ultrafast X-ray scattering," Science 316, 1444 (2007).

${ }^{2}$ R. Neutze, R. Wouts, D. van der Spoel, E. Weckert, and J. Hajdu, "Potential for biomolecular imaging with femtosecond X-ray pulses," Nature (London) 406, 752 (2000).

${ }^{3}$ M. Hoener, L. Fang, O. Kornilov, O. Gessner, S. T. Pratt, M. Guehr, E. P. Kanter, C. Blaga, C. Bostedt, J. D. Bozek, P. H. Bucksbaum, C. Buth, M. Chen, R. Coffee, J. Cryan, L. F. DiMauro, M. Glownia, E. Hosler, E. Kukk, S. R. Leone, B. McFarland, M. Messerschmidt, B. Murphy, V. Petrovic, D. Rolles, and N. Berrah, "Ultraintense X-ray induced ionization, dissociation, and frustrated absorption in molecular nitrogen, Phys. Rev. Lett. 104, 253002 (2010).

${ }^{4}$ L. Young, E. P. Kanter, B. Kraessig, Y. Li, A. M. March, S. T. Pratt, R. Santra, S. H. Southworth, N. Rohringer, L. F. DiMauro, G. Doumy, C. A Roedig, N. Berrah, L. Fang, M. Hoener, P. H. Bucksbaum, J. P. Cryan, S. Ghimire, J. M. Glownia, D. A. Reis, J. D. Bozek, C. Bostedt, an M. Messerschmidt, "Femtosecond electronic response of atoms to ultraintense X-rays," Nature (London) 466, 56 (2010).

${ }^{5}$ L. S. Cederbaum, F. Tarantelli, A. Sgamellotti, and J. Schirmer, "On double vacancies in the core," J. Chem. Phys. 85, 6513 (1986).

${ }^{6} \mathrm{~N}$. V. Kryzhevoi, M. Tashiro, M. Ehara, and L. S. Cederbaum, "Interatomic relaxation effects in double core ionization of chain molecules," J. Chem. Phys. 137, 154316 (2012)

N. V. Kryzhevoi, R. Santra, and L. S. Cederbaum, "Inner-shell single an double ionization potentials of aminophenol isomers," J. Chem. Phys. 135, $084302(2011)$

${ }^{8}$ M. Tashiro, M. Ehara, H. Fukuzawa, K. Ueda, C. Buth, N. V. Kryzhevoi, and L. S. Cederbaum, "Molecular double core hole electron spectroscopy for chemical analysis," J. Chem. Phys. 132, 184302 (2010).

${ }^{9}$ M. Tashiro, K. Ueda, and M. Ehara, "Auger decay of molecular double core-hole state," J. Chem. Phys. 135, 154307 (2011).

${ }_{10}^{10} \mathrm{~J}$. H. D. Elat, ' . Chem. Phys. 135, 154307 (2011). "Double core hole creation and subsequent auger decay in $\mathrm{NH}_{3}$ and $\mathrm{CH}_{4}$ "Double core hole creation and subsequent auger

${ }^{11}$ P. Kolorenč and V. Averbukh, "K-shell Auger lifetime variation in doubly "P. Kolorenć and V. Averbukh, "K-shell Auger lifetime variation in doubly
ionized Ne and first row hydrides," J. Chem. Phys. 135, 134314 (2011). 12 J. Cryan, J. Glownia, J. Andreasson, A. Belkacem, N. Berrah, C. Blaga,
1 . ${ }^{12}$ J. Cryan, J. Glownia, J. Andreasson, A. Belkacem, N. Berrah, C. Blaga,
C. Bostedt, J. Bozek, C. Buth, L. DiMauro, L. Fang, O. Gessner, M. C. Bostedt, J. Bozek, C. Buth, L. DiMauro, L. Fang, O. Gessner, M Guehr, J. Hajdu, M. Hertlein, M. Hoener, O. Kornilov, J. Marangos, A
March, B. McFarland, H. Merdji, V. Petrovic, C. Raman, D. Ray, D. Reis, March, B. McFarland, H. Merdji, V. Petrovic, C. Raman, D. Ray, D. Reis, and R. Coffee, "Auger electron angular distribution of double core-hole states in the molecular reference frame," Phys. Rev. Lett. 105, 083004 (2010).
${ }^{13} \mathrm{M}$. H. Chen, "Auger transition rates and fluorescence yields for the double$K$-hole state," Phys. Rev. A 44, 239 (1991).

${ }^{14}$ L. Inhester, C. F. Burmeister, G. Groenhof, and H. Grubmuller, "Auger spectrum of a water molecule after single and double core ionization," J. Chem. Phys. 136, 144304 (2012)

${ }^{15}$ C. P. Bhalla, N. O. Folland, and M. A. Hein, "Theoretical $K$-Shell auger rates, transition energies, and fluorescence yields for multiply ionized neon," Phys. Rev. A 8, 649 (1973).

${ }^{16} \mathrm{~F}$. Larkins, "Influence of core hole screening on molecular auger rates and inner-shell lifetimes," J. Electron Spectrosc. Relat. Phenom. 67, 159 (1994).

${ }^{17}$ P. Demekhin, A. Ehresmann, and V. Sukhorukov, "Single center method: A computational tool for ionization and electronic excitation studies of A computational tool for ionization and electron
molecules," J. Chem. Phys. 134, 024113 (2011).

molecules," J. Chem. Phys. 134, 024113 (2011).
${ }^{18}$ R. Gaspar, Über eine approximation des hartreefockschen potentials durch ${ }^{18}$ R. Gaspar, Über eine approximation des hartreefockschen potentials durch
eine universelle potentialfunktion," Acta Phys. Acad. Sci. Hung. 3, 263 (1954)

${ }^{19}$ M. J. Frisch, G. W. Trucks, H. B. Schlegel et al., Gaussian 09, Revision A.02, Gaussian, Inc., Wallingford, CT, 2009.

${ }^{20}$ J. A. Pople, R. Krishnan, H. B. Schlegel, and J. S. Binkley, "Derivative studies in Hartree-Fock and Mller-Plesset theories," Int. J. Quantum Chem. 16, 225 (1979).

${ }^{21}$ T. H. Dunning, "Gaussian basis sets for use in correlated molecular calculations. I. The atoms boron through neon and hydrogen," J. Chem. Phys. 90, 1007 (1989)

${ }^{22}$ T. D. Crawford, C. D. Sherrill, E. F. Valeev, J. T. Fermann, R. A. King, M. L. Leininger, S. T. Brown, C. L. Janssen, E. T. Seidl, J. P. Kenny, and W. D. Allen, "PSI3: An open-source ab initio electronic structure package," J. Comput. Chem. 28, 1610 (2007).

${ }^{23}$ T. X. Carroll, N. Berrah, J. Bozek, J. Hahne, E. Kukk, L. J. Sæthre, and T. D. Thomas, "Carbon $1 s$ photoelectron spectrum of methane: Vibrational T. D. Thomas, "Carbon $1 s$ photoelectron spectrum of methane:
excitation and core-hole lifetime," Phys. Rev. A 59, 3386 (1999).

${ }^{24}$ H. M. Köppe, B. S. Itchkawitz, A. L. D. Kilcoyne, J. Feldhaus, B. Kemp-
(a) H. M. Köppe, B. S. Itchkawitz, A. L. D. Kilcoyne, J. Feldhaus, B. Kemp-
gens, A. Kivimäki, M. Neeb, and A. M. Bradshaw, "High-resolution c $1 s$ gens, A. Kivimäki, M. Neeb, and A. M. Bradshaw, "High-resolution c
photoelectron spectra of methane," Phys. Rev. A 53, 4120 (1996).

${ }^{25}$ photoelectron spectra of methane," Phys. Rev. A 53, 4120 (1996).
${ }^{2}$. Sankari, M. Ehara, H. Nakatsuji, Y. Senba, K. Hosokawa, H. Yoshida, ${ }^{25}$ R. Sankari, M. Ehara, H. Nakatsuji, Y. Senba, K. Hodokawa, H. Yoshida,
A. D. Fanis, Y. Tamenori, S. Aksela, and K. Ueda, "Vibrationally resolved O 1s photoelectron spectrum of water," Chem. Phys. Lett. 380, 647 (2003).

${ }^{26} \mathrm{R}$. Manne and H. Ågren, "Auger transition amplitudes from general manyelectron wavefunctions," Chem. Phys. 93, 201 (1985).

${ }^{27}$ H. Siegbahn, L. Asplund, and P. Kelfve, "The Auger electron spectrum of water vapour," Chem. Phys. Lett. 35, 330 (1975).

${ }^{28} \mathrm{M}$. Coville and T. D. Thomas, "Molecular effects on inner-shell lifetimes: Possible test of the one-center model of auger decay,” Phys. Rev. A 43, 6053 (1991).

${ }^{29}$ D. E. Ramaker, J. S. Murday, N. H. Turner, G. Moore, M. G. Lagally, and J. Houston, "Calculated and measured auger line shapes in $\mathrm{sio}_{2}$," Phys. Rev. B 19, 5375 (1979).

${ }^{30}$ D. E. Ramaker, "Final-state correlation effects in auger line shapes: Application to $\mathrm{SiO}_{2}$," Phys. Rev. B 21, 4608 (1980).

${ }^{31}$ cation to $\mathrm{SiO}_{2}$," Phys. Rev. B 21, 4608 (1980). ${ }^{31}$ F. Larkins, "Theoretical interpretation of molec

tron Spectrosc. Relat. Phenom. 51, 115 (1990). M. Mitani, O. Takahashi, K. Saito, and S. Iwata, "Theoretical molecular Auger spectra with electron population analysis," J. Electron Spectrosc Relat. Phenom. 128, 103 (2003).

${ }^{33} \mathrm{D}$. Walters and C. Bhalla, "Nonrelativistic k-shell auger rates and matrix elements for $4<\mathrm{z} \leq 54$," At. Data Nucl. Data Tables 3, 301 (1971).

${ }^{34}$ M. H. Chen, F. P. Larkins, and B. Crasemann, "Auger and coster-kronig radial matrix elements for atomic numbers $6 \leq \mathrm{z} \leq 92$," At. Data Nucl. Data Tables 45, 1 (1990).

${ }^{35}$ P.-O. Lowdin, "On the non-orthogonality problem connected with the use of atomic wave functions in the theory of molecules and crystals," J. Chem. Phys. 18, 365 (1950). 


\subsection{Auger spectrum of acetylene after single and double core ionization}

\subsubsection{Introduction}

So far, I considered only core hole vacancies in molecules with one K-shell in this thesis. Now, I will study a molecule with two heavier nuclei, specifically, I consider the Auger decay after single and double core ionization in acetylene $\left(\mathrm{C}_{2} \mathrm{H}_{2}\right)$. Acetylene was selected as it is one of the simplest hydrocarbons and thus represents a starting point for future studies of hydrocarbons. Furthermore, the linear symmetry of this molecule (point group symmetry $D_{\infty h}$ ) simplifies electronic structure calculations considerably. With respect to the Auger decay, the situation in this molecule is different compared with the molecules studied in the previous sections, because here the two core orbitals are delocalized over both carbon atoms. The molecular orbital structure is sketched in Fig. 3.8. As can be seen, acetylene contains 14 electrons and the energetically highest occupied molecular orbital is the $\pi_{u}$ orbital, which forms together with the $3 \sigma_{g}$ molecular orbital the triple-bond. The two core orbitals, one with gerade and one with ungerade parity, are energetically nearly degenerate, which give rise to two energetically close lying single core ionized states. The K-LL Auger decay originating from these two core ionized states have been studied before experimentally by Kivimäki et al. [57] and theoretically by Colle et al. [21].

Double core ionization of acetylene gives rise to two distinct types of Auger transitions originating from double ionization on the same nuclear site (single-site double core hole) or on different nuclear sites (two-site double core hole). Recently, the Auger spectra corresponding to these two types of double core ionization in acetylene has been measured by Lablanquie et al. 63 via single-photon double core ionization using multicoincidence techniques. In calculations, these Auger decay transitions have - up to my knowledge - only been studied based on population analysis [91, and calculations of the absolute double core hole decay rate have not been performed, yet. As motivated in the introduction (chapter 1), these quantities provide important insight into the radiation damage mechanism of a molecule exposed to the high intense X-ray pulse provided by XFELs, as the refilling of a core vacancy determines how fast subsequent ionization occurs.

In the following subsections, I will show how the decay rates for the two double core hole types in acetylene were obtained. Further, I will demonstrate that the core hole screening effects, studied in the previous section 3.3 for molecules with a single K-shell [51], have 
dramatic effects on the decay rate of these core hole vacancies. Closer inspection of the electron densities of the single-site and two-site double core hole states illustrates that the two types of double core vacancies have considerably different impact on the valence electronic structure. To assess my calculations against previous studies $13,21,22,55$, 57 I will also present results regarding the K-LL Auger spectrum originating from single core ionization.
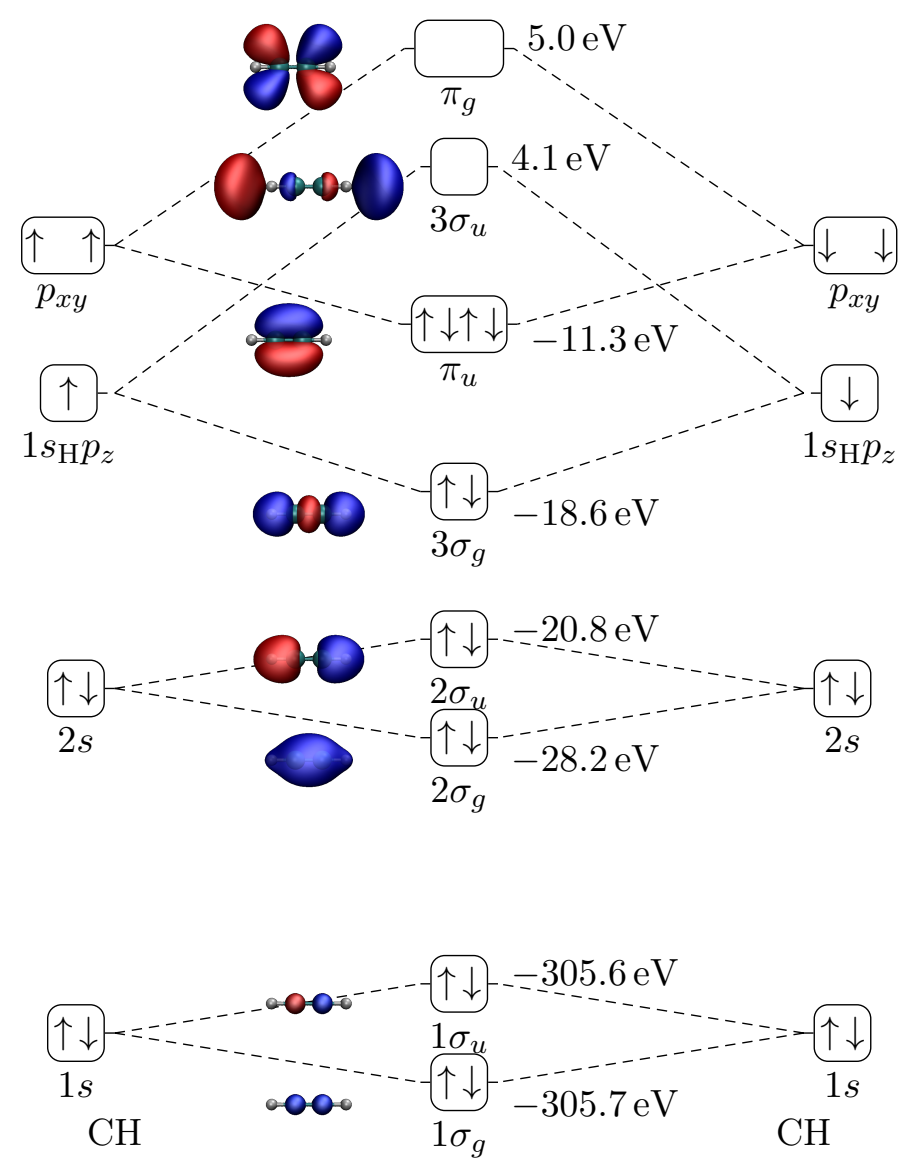

Figure 3.8: Molecular orbital diagram of acetylene $\left(\mathrm{C}_{2} \mathrm{H}_{2}\right)$ with orbital energies from a neutral state RHF calculation for the occupied and the first unoccupied molecular orbitals. 


\section{Results}

\subsubsection{Computational details}

For all the calculation performed for this section, I used molecular orbitals obtained in a Hartree-Fock calculation employing the cc-pVTZ basis set [95]. The molecular geometry was taken from a MP2 geometry optimization performed with the GAUSSIAN09 quantum package [36. Initial and final electronic bound states were calculated with configuration interaction calculations using multiple references. Details on the specific choice of reference and truncation scheme are given below.

For the single center expansion, I used an adapted truncation of $l, m$ angular quantum numbers. Orienting the molecule along the $z$ axis, the angular quantum number $m$ is a good quantum number. Keeping in mind that acetylene has two electrons in a $\pi$ orbital $(m= \pm 1)$, the Auger electron can have a $m$ quantum number for which $|m| \leq 2$. Thus, I restricted the calculation to include only spherical harmonics of $|m| \leq 2$. The center of the expansion was chosen to be at the molecular center of mass. To decide at which angular quantum number $l$ the calculation should be truncated, the individual contributions of the molecular orbitals for a specific $l$ quantum number were inspected. Figure 3.9 shows the partial norm $\int_{0}^{\infty} d r\left|P_{i, l m}(r)\right|^{2}$ as a function of the $l$ quantum number for the relevant orbitals, which were obtained in a $\mathrm{ROHF}$ calculation for the $1 \sigma_{g}$ vacancy state. As can be seen, for each orbital the contributions decline rapidly with $l$, such that for $l \geq 10$ only small contributions are found mainly for the two core orbitals. The Auger electron may carry away at maximum an angular momentum of two bound electrons, thus an angular quantum number truncation of $l \leq 20$ was used. For the radial expansion, a total number of 2000 radial grid points was used with a maximal grid point at 20 a.u. 

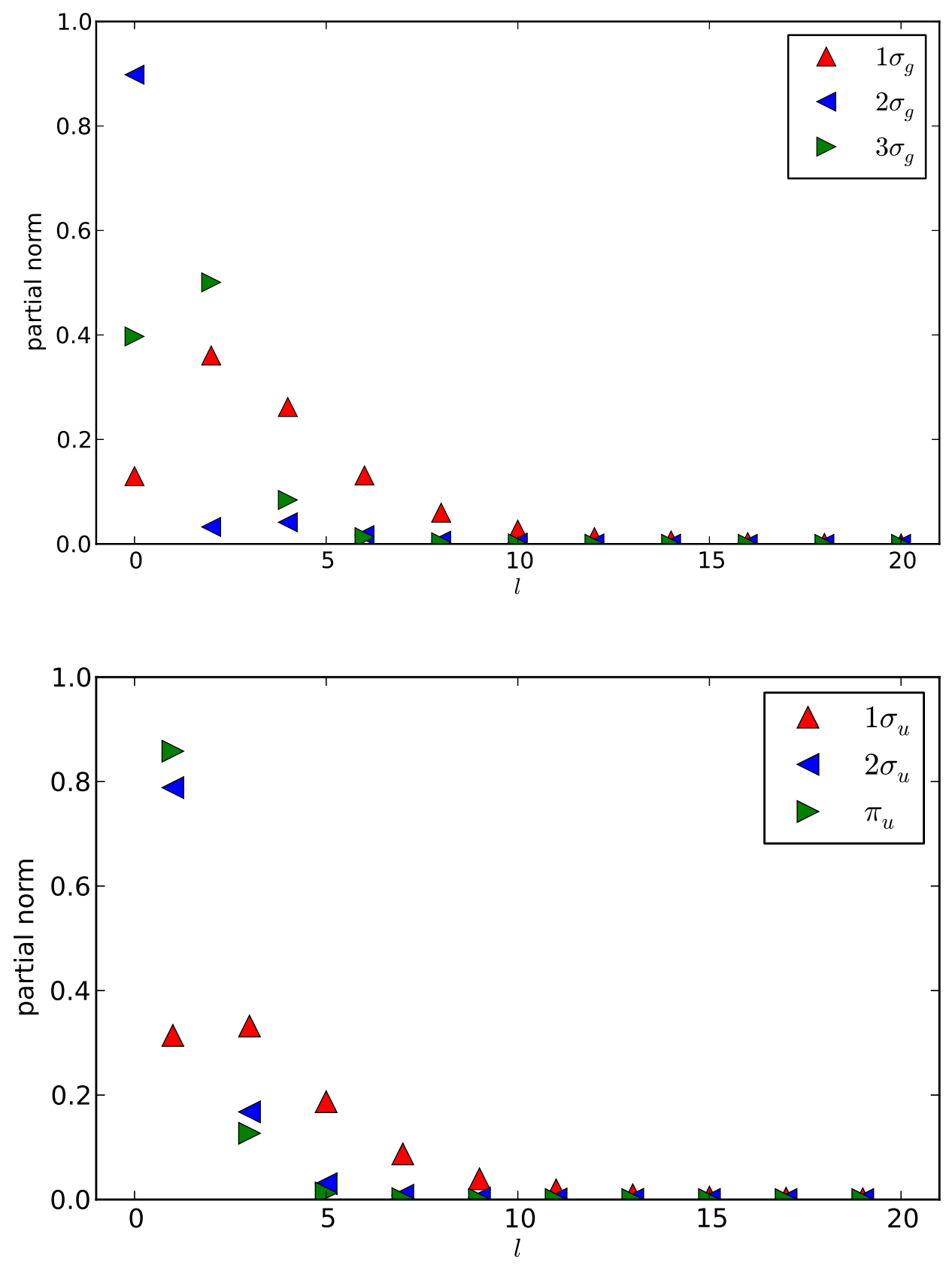

Figure 3.9: Partial norm $\left(\int_{0}^{\infty} d r\left|P_{i, l m}(r)\right|^{2}\right)$ of the molecular orbitals of acetylene as a function of the angular quantum number $l$. The upper figure shows orbitals with gerade parity, the lower figure shows orbitals with ungerade parity. For all considered molecular orbitals the partial norm declines rapidly with $l$. 


\subsubsection{Auger spectrum after single core ionization $(\mathrm{K}-\mathrm{LL})$}

Two different kinds of K-LL Auger decay transitions arise from the two core holes, $1 \sigma_{g}$ and $1 \sigma_{u}$, which are investigated here individually. Unless a resonant Auger process is considered, in which the core electron is initially not ionized but excited to a higher lying molecular orbital (e.g. $1 \sigma_{g} \rightarrow \pi_{g}$ ), it is impossible to discern between these two cases experimentally. Instead, the initial ionization creates in general a linear combination of both vacancies states. The interferences in the resulting Auger spectrum originating from the linear combination of these two core hole states influences the angular distribution of the emitted Auger electron. In this calculation, the angular averaged spectrum is considered in which these interferences cancel out because of their different parity [22]. It is therefore justified to treat these two core hole states independently. The resonant and non-resonant K-LL Auger spectra of acetylene have been studied before, both experimentally [57] and by ab-initio quantum chemical calculations [21, 22], in which a basis set of hermite Gaussian functions was used to represent the electronic continuum. Thus, in the following, I will assess how well these results compare with my calculations.

\section{Computational parameters for the $\mathrm{Cl}$ calculation}

For the computation of the two Auger spectra, I used molecular orbitals which were obtained from a restricted open shell Hartree-Fock calculation (ROHF) of the $1 \sigma_{g}$ and $1 \sigma_{u}$ vacancy state, respectively. The ROHF calculations were performed using the PSI3 quantum package 24. The two core vacancy states, $1 \sigma_{g}^{-1}$ and $1 \sigma_{u}^{-1}$, were then obtained using a multi-reference configuration interaction calculation, with both desired occupations as references. All single and double excitations from these references have been included in the calculation (CISD) with the additional restriction that there are three electrons in both core orbitals $\left(1 \sigma_{g}\right.$ and $\left.1 \sigma_{u}\right)$. For the final electronic states, I used a CI truncation including all single and double excitations from multiple references. These references represent all possible combinations of two valence orbital vacancies.

\section{Results}

In Figure 3.10, the calculated K-LL Auger decay transitions are compared with the experimental spectrum from Ref. [57]. To facilitate the direct comparison, all calculated 
transitions have been downshifted by $2.3 \mathrm{eV}$. As discussed in section 3.1 , this shift may result from the CI truncation, the limited basis set, and neglected relativistic effects. In line with previous studies [21], minor differences in the transition rates between the $1 \sigma_{g}$ and $1 \sigma_{u}$ hole occur. The calculated transitions for the $1 \sigma_{g}$ hole are convoluted by a Gaussian profile with FWHM $=3.5 \mathrm{eV}$ to mimic the broadening due to the vibrational effects and the finite detector band width. As can be seen, the features of the experimental spectrum are much broader in the low energy range of the spectrum compared to the high energy part. This increase in broadening cannot be solely explained by the higher density of inner valence vacancy states in the low energy range of the spectrum. A similar behavior has been found for the water molecule (see section 3.2 ) and is explained by taking into account the vibrational broadening more explicitly. This analogy suggests that the inner valence hole states see a much steeper potential energy surface, such that the lower part of the spectrum is subject to a much stronger vibrational broadening. An investigation of the vibrational broadening for each individual transition may thus considerably improve the agreement with the experimental spectrum and will be adressed in future studies.

The total decay rate of the core hole was found to be $2.93 \cdot 10^{-3}$ a.u. for the $1 \sigma_{g}$ hole and $3.04 \cdot 10^{-3}$ a.u. for the $1 \sigma_{u}$ hole. These values are in good agreement with the values calculated by Colle et al. 21] $\left(2.94 \cdot 10^{-3}\right.$ a.u. and $2.5 \cdot 10^{-3}$ a.u. $)$ and comply with the line widths extracted from photo-ionization spectra $\left(3.9 \pm 0.1 \cdot 10^{-3}\right.$ a.u. 13 and $3.3 \pm 0.3$. $10^{-3}$ a.u. [55]). Also the previously [51] calculated value for methane $\left(3.2 \cdot 10^{-3}\right.$ a.u. $)$ compares well with the here calculated decay rates for acetylene.

In summary, the overall spectrum is well reproduced by the calculated transition rates originating from both, the $1 \sigma_{g}$ and the $1 \sigma_{u}$ core holes. The numerical procedure quantitatively reproduces experimental results. This agreement also supports the choice of computational parameters for the considerations in the following subsection regarding the KK-KLL Auger spectrum. 


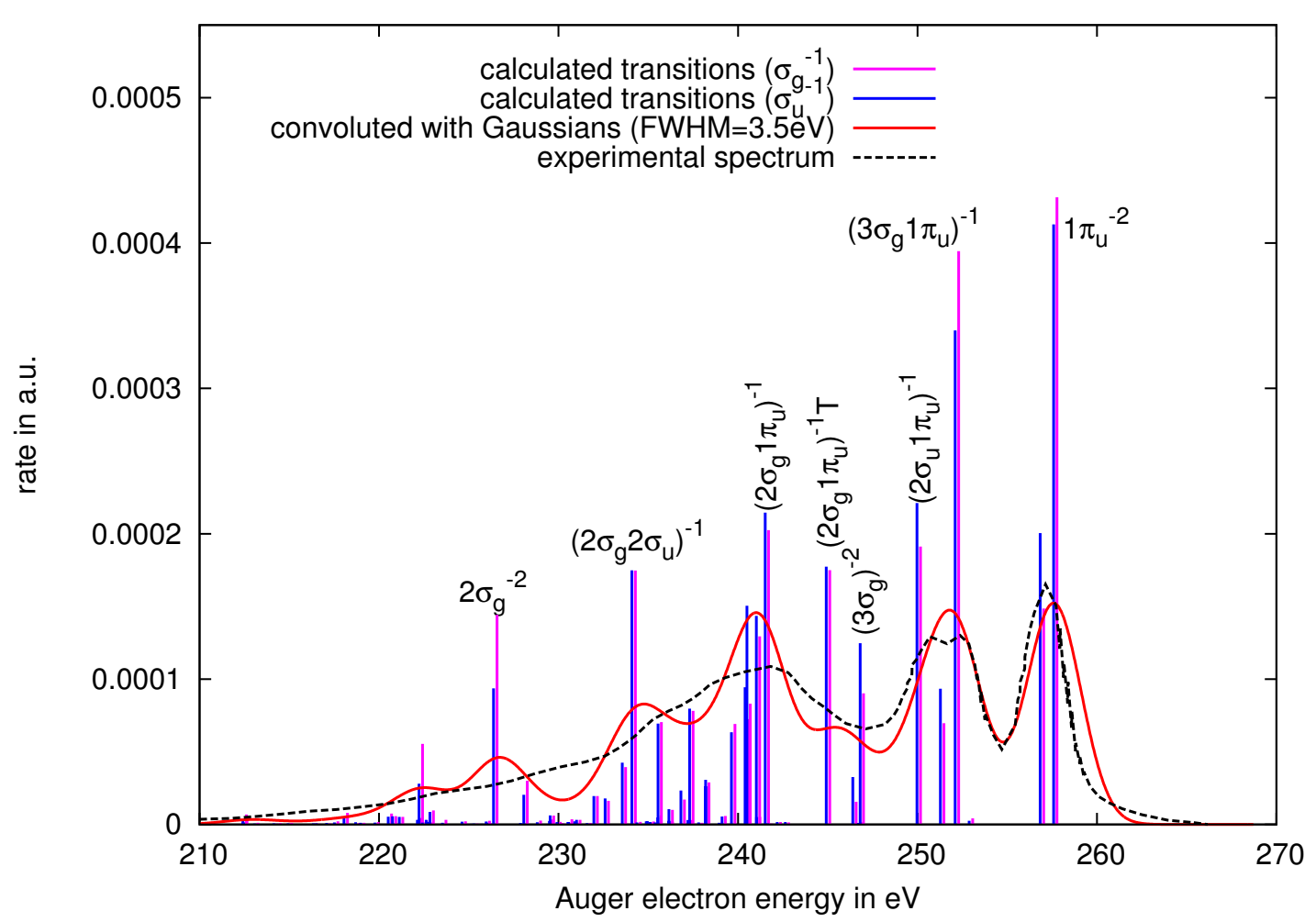

Figure 3.10: Calculated K-LL Auger transitions for acetylene compared with the experimentally determined spectrum from Ref. [57] (dashed line). The magenta (blue) vertical lines show the transitions originating from the $1 \sigma_{g}\left(1 \sigma_{u}\right)$ hole. Calculated transition energies have been downshifted by $2.3 \mathrm{eV}$. The major decay transitions have been labeled according to their dominant final valence hole configuration, where $\mathrm{T}$ denotes a triplet final state configuration. The red line shows the calculated transitions of the $\sigma_{g}$ hole convoluted with a Gaussian function with $3.5 \mathrm{eV}$ FWHM. 


\subsubsection{Auger spectrum after double core ionization (KK-KLL)}

For the KK-KLL Auger transition, the following combination of double K-holes arise in acetylene:

$$
\begin{aligned}
\psi_{\Sigma_{g},+} & =\frac{1}{\sqrt{2}}\left(\left(1 \sigma_{g}\right)^{-2}+\left(1 \sigma_{u}\right)^{-2}\right), \\
\psi_{\Sigma_{g},-} & =\frac{1}{\sqrt{2}}\left(\left(1 \sigma_{g}\right)^{-2}-\left(1 \sigma_{u}\right)^{-2}\right), \\
\psi_{\Sigma_{\Sigma_{u},+}} & =\frac{1}{\sqrt{2}}\left(\left(1 \sigma_{g, \uparrow} 1 \sigma_{u, \downarrow}\right)^{-1}+\left(1 \sigma_{u, \uparrow} 1 \sigma_{g, \downarrow}\right)^{-1}\right), \\
\psi_{\Sigma_{\Sigma_{u},-}} & =\frac{1}{\sqrt{2}}\left(\left(1 \sigma_{g, \uparrow} 1 \sigma_{u, \downarrow}\right)^{-1}-\left(1 \sigma_{u, \uparrow} 1 \sigma_{g, \downarrow}\right)^{-1}\right) .
\end{aligned}
$$

The first three are singlet states $\left({ }^{1} \Sigma_{g},+,{ }^{1} \Sigma_{g},-\right.$ and $\left.{ }^{1} \Sigma_{u},+\right)$, the last one is a triplet state $\left({ }^{3} \Sigma_{u},-\right)$. Rewriting the molecular core orbitals as

$$
\phi_{1 \sigma_{g}}=\frac{1}{\sqrt{2}}\left(\phi_{1 s_{a}}+\phi_{1 s_{b}}\right), \quad \text { and } \quad \phi_{1 \sigma_{u}}=\frac{1}{\sqrt{2}}\left(\phi_{1 s_{a}}-\phi_{1 s_{b}}\right),
$$

where $\phi_{1 s_{a}}$ and $\phi_{1 s_{b}}$ are now atomic core orbitals localized on carbon atom $a$ and $b$, respectively, yields

$$
\begin{aligned}
& \psi_{\Sigma_{\Sigma_{g},+}}=\frac{1}{\sqrt{2}}\left(\left(1 s_{a}\right)^{-2}+\left(1 s_{b}\right)^{-2}\right), \\
& \psi_{\Sigma_{\Sigma_{g},-}}=\frac{1}{\sqrt{2}}\left(\left(1 s_{a}, \uparrow 1 s_{b}, \downarrow\right)^{-1}+\left(1 s_{b}, \uparrow 1 s_{a}, \downarrow\right)^{-1}\right), \\
& \psi_{\Sigma_{\Sigma_{u},+}}=\frac{1}{\sqrt{2}}\left(\left(1 s_{a}\right)^{-2}-\left(1 s_{b}\right)^{-2}\right), \\
& \psi_{3 \Sigma_{u},-}=\frac{1}{\sqrt{2}}\left(\left(1 s_{a}, \uparrow 1 s_{b}, \downarrow\right)^{-1}-\left(1 s_{b}, \uparrow 1 s_{a}, \downarrow\right)^{-1}\right) .
\end{aligned}
$$

From these equations one can infer, that the first and the third singlet states $\left({ }^{1} \Sigma_{g},+\right.$ and $\left.{ }^{1} \Sigma_{u},+\right)$ represent a linear combination of hole states, in which both K-holes are located on the same nuclear site. In contrast, the second singlet and the triplet state $\left({ }^{1} \Sigma_{g},-\right.$ and $\left.{ }^{3} \Sigma_{u},-\right)$ represent hole states with both K-holes located on different nuclear sites 17 .

Although the $1 \sigma_{g}$ and $1 \sigma_{u}$ orbitals are nearly degenerate, the two types of combinations, the single-site and the two-site double core holes, are energetically well separated mainly due to the electrostatic repulsion between the two holes. Thus, the spectroscopic 


\section{Results}

analysis of these different types of molecular double core holes provide sensitive information on the distance of the two involved core holes and thereby on the molecular and electronic structure [82, 90].

\section{Computational details of the $\mathrm{Cl}$ calculation}

For the calculations of all KK-KLL Auger transitions, I used molecular orbitals which were obtained by a ROHF calculation performed with the PSI3 quantum package 24 in which each of the $1 \sigma_{g}$ and $1 \sigma_{u}$ orbitals was singly occupied. With these molecular orbitals, initial and final states were calculated in a configuration interaction calculation setup similar to that used for the calculation of the K-LL Auger transitions. In particular, single and double excitations from multiple references that represent all double vacancies in the core were used for the initial states with the additional restriction of having always two electrons in the two core orbitals. The final electronic states were obtained from configuration interaction calculations including all single and double excitations from multiple references that represent all combinations of having two vacancies in the valence orbitals. Additionally, it was required that in all configurations there are three electrons in the two core orbitals. Whereas the singlet states always decay into final doublet states, for the decay of the triplet double core hole state ${ }^{3} \Sigma_{u}$, - also final quadruplet states were calculated.

\section{Results}

The calculated KK-KLL Auger transitions for the single-site core hole states are shown in Fig. 3.11. As for the K-LL transitions, the transition energies have been downshifted by $2.3 \mathrm{eV}$ to yield a better agreement with the experimental spectrum. Both singlesite core hole states ${ }^{1} \Sigma_{g},+$ and ${ }^{1} \Sigma_{u},+$ reveal slight differences in some transition rates, which is in line with the observation for the K-LL Auger transitions. The Auger decay transitions originating from the ${ }^{1} \Sigma_{g},+$ double core hole state convoluted with Gaussian functions with FWHM $3.5 \mathrm{eV}$ are in good agreement with the measured spectrum. As observed for the K-LL Auger spectrum, the spectral features tend to be broader in the low energy range. Closer inspection of the potential energy surfaces might also provide a more reliable convolution scheme for incorporating the vibrational broadening here.

Direct comparison of the calculated KK-KLL Auger decay transitions for the twosite double core hole states with the experimental spectrum is more difficult, as the 
experimental spectrum for these transitions is vague. The experimental detection of the two-site double core Auger electrons is much more challenging, as the probability for a two-site double core hole via single-photon ionization is much lower, namely only $1.6 \%$ with respect to single-site double core ionization [63]. Furthermore, the energies of the emitted Auger electrons overlap with those originating from a single K-hole. The resulting experimental values have therefore very large error bars. Figure 3.12 shows the calculated KK-KLL Auger transitions compared to the experimental KK-KLL Auger spectrum for the two-site double core hole states. As observed for the single-site KK-KLL Auger decay and the K-LL Auger decay, slight differences occur in the calculated transitions for the two nearly degenerate core hole states ${ }^{1} \Sigma_{g},-$ and ${ }^{3} \Sigma_{u},-$. The convoluted transition rates, here again shifted by $2.3 \mathrm{eV}$, are in agreement with the experimental spectrum.

Table 3.3: Calculated total transition rates $\Gamma^{\text {Auger }}$ and life times $\tau$ for double K-holes in acetylene.

\begin{tabular}{c|l|l} 
core hole state & $\Gamma^{\text {Auger }}$ in $10^{-3}$ a.u. & $\tau$ in fs \\
\hline${ }^{1} \Sigma_{g},+$ & 11.79 & 2.1 \\
${ }^{1} \Sigma_{u},+$ & 11.79 & 2.1 \\
${ }^{1} \Sigma_{g},-$ & 5.20 & 4.6 \\
${ }^{3} \Sigma_{u},-$ & 5.16 & 4.6
\end{tabular}

The total decay rates obtained for all four double core hole states are listed in Table 3.3 . It can be seen, that the decay rates within the single-site and the two-site double core holes are pairwise almost equal. The previously calculated total decay rate for double core ionized methane $\left(11.6 \cdot 10^{-3}\right.$ a.u. $)[51]$ is in good agreement with the decay rates of the single-site double core holes. Remarkably, the decay rates of the single-site double core holes $\left({ }^{1} \Sigma_{g},+\right.$ and $\left.{ }^{1} \Sigma_{u},+\right)$ are much larger compared with the decay rates of the two-site core holes $\left({ }^{1} \Sigma_{g},-\right.$ and $\left.{ }^{3} \Sigma_{u},-\right)$.

This observation is in line with the findings in section 3.3, which explains the enhancement of Auger decay rates as a result of the core hole screening effects. A double core hole located on the same nuclear site causes strong screening effects, such that valence electrons from the non-ionized carbon nucleus are transferred towards the core hole. In contrast, for core holes located on different sites, these screening effects are non-cooperative and thus much weaker. The increased valence electron density results in a much larger number of valence electrons that are in the vicinity of the core hole and thus available for recombination in the Auger decay. Therefore, the decay rate in the single-site double core holes is much higher. 
To investigate this core hole screening mechanism in more detail, I now compare the different electron density distributions for the different core hole states. To perform this analysis for the single-site double core holes, a wave function with localized core holes (both located on carbon atom a) was considered, which is given by the linear combination

$$
\psi_{1 s_{a}-2}=\frac{1}{\sqrt{2}}\left(\psi_{1 \Sigma_{g},+}+\psi_{1 \Sigma_{u},+}\right) .
$$

The electron density distribution of this wave function $\rho_{1 s_{a}{ }^{-2}}(\mathbf{r})$ reads in terms of the electron density distributions of the two states ${ }^{1} \Sigma_{g},+$ and ${ }^{1} \Sigma_{u},+$

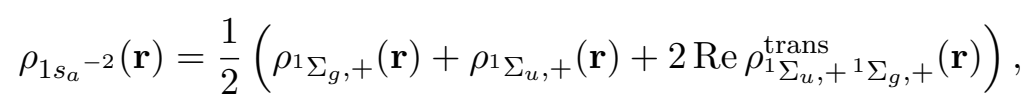

where $\rho_{1 \Sigma_{g},+}(\mathbf{r})$ and $\rho_{1 \Sigma_{u},+}(\mathbf{r})$ are the electron density distributions of the ${ }^{1} \Sigma_{g},+$ and

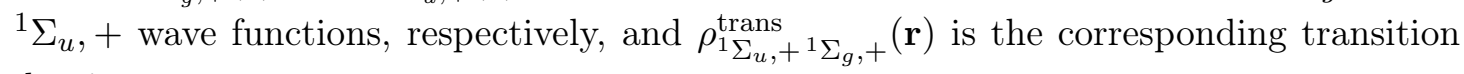
density.

Accordingly, for the single core hole, I consider the electron density distribution from the linear combination of both, the $\sigma_{g}^{-1}$ and $\sigma_{u}^{-1}$ core hole states,

$$
\psi_{1 s_{a}-1}=\frac{1}{\sqrt{2}}\left(\psi_{2 \Sigma_{g}}+\psi_{2 \Sigma_{u}}\right)
$$

from which the electron density is derived accordingly. The resulting electron densities are illustrated in Fig. 3.13, where a planar cut through the electron density containing the molecular axis is shown. As anticipated, the single-site double core hole results in much stronger valence electron rearrangements than those observed for the other core hole states. The stronger screening effect is also quantified by the number of valence electrons located on the respective core ionized carbon atom as estimated by the Mulliken and Löwdin population analysis. The resulting valence electron populations are shown in Table 3.4

In summary, single-site double core holes in acetylene have a much stronger decay rate than two-site double core holes. This is explained by the different core hole screening effects, which are cooperative in the single-site case and anti-cooperative in the two-site case. 
Table 3.4: Valence electron population on the respective core hole site for the neutral, single, and double core ionized states of acetylene obtained by Mulliken and Löwdin population analysis.

\begin{tabular}{r|l|l} 
Core hole state & Mulliken & Löwdin \\
\hline Neutral & 4.30 & 4.15 \\
Single core hole & 4.83 & 4.64 \\
Double core hole (two-site) & 4.41 & 4.29 \\
Double core hole (single-site) & 5.56 & 5.69
\end{tabular}

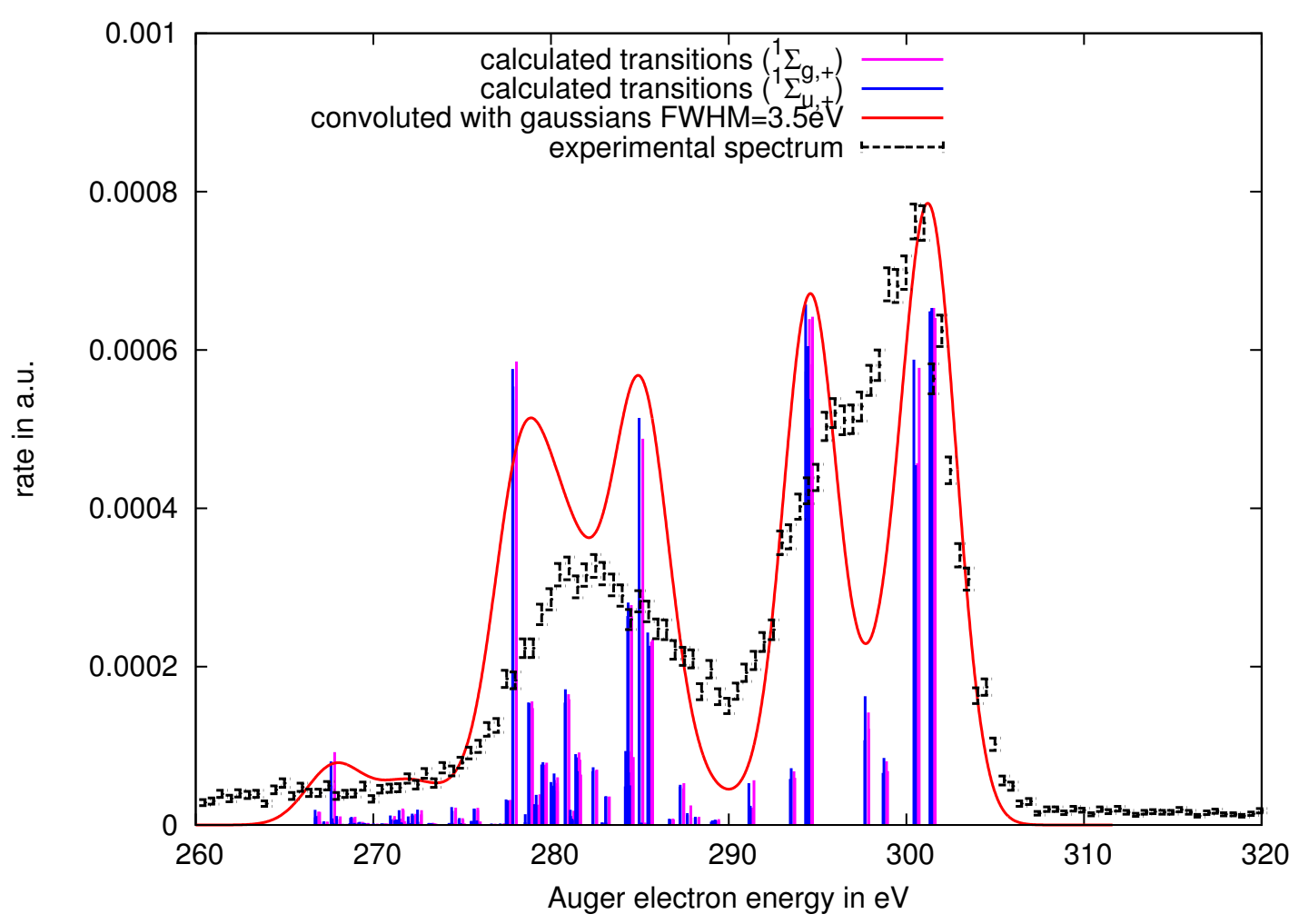

Figure 3.11: Calculated KK-KLL Auger transitions compared with the measured spectrum for the single-site core hole states. The calculated transition rates are denoted by magenta and blue vertical lines for the ${ }^{1} \Sigma_{g},+$ and ${ }^{1} \Sigma_{u},+$ double core hole states, respectively. Calculated transition energies have been downshifted by $2.3 \mathrm{eV}$. The experimental spectrum was taken from Ref. 92 (black error bars). The calculated transitions originating from the ${ }^{1} \Sigma_{g},+$ double core hole state have been convoluted with a Gaussian function with $3.5 \mathrm{eV}$ FWHM (red line). 


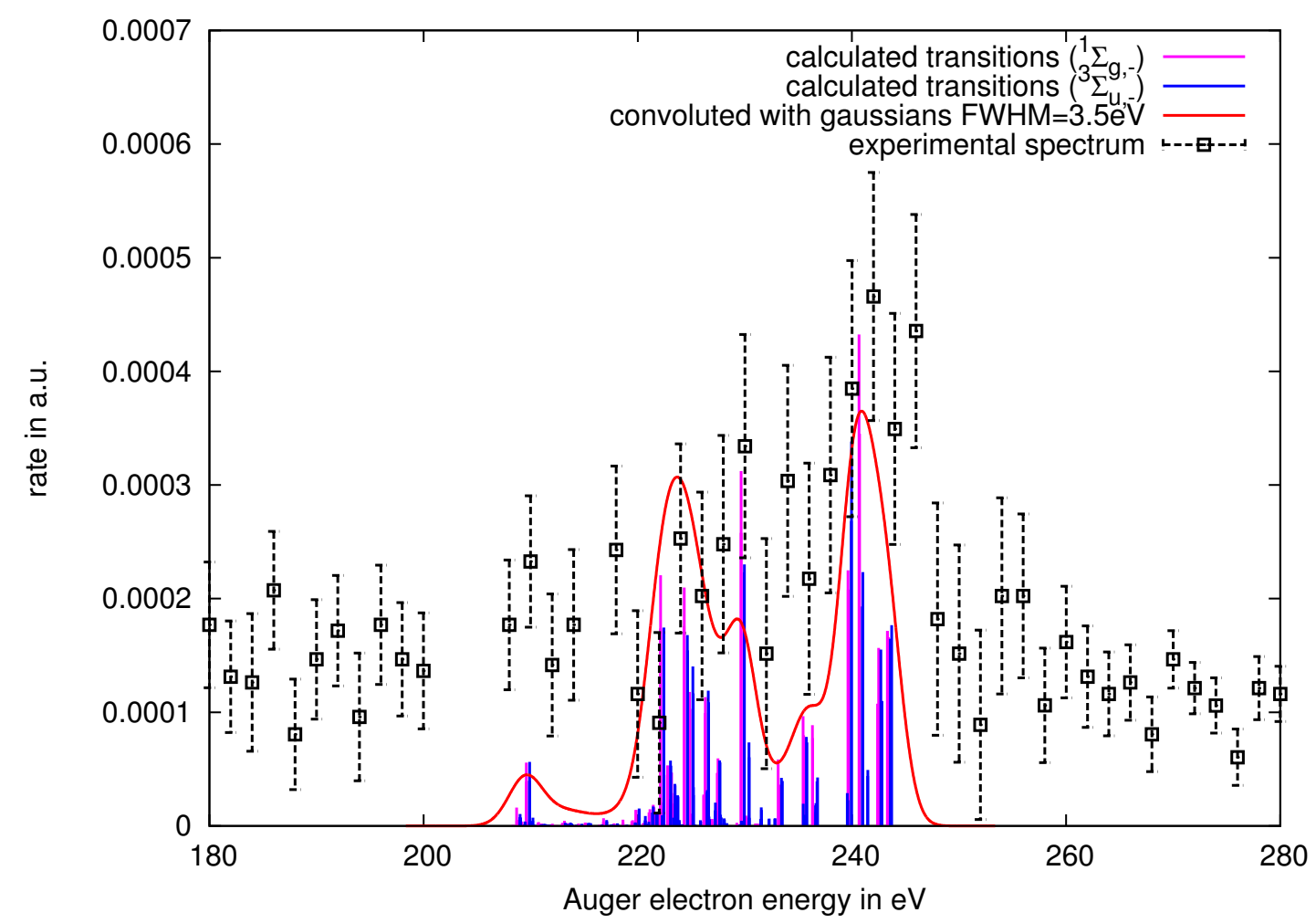

Figure 3.12: Calculated KK-KLL Auger transitions compared with experimental spectrum for the two-site double core hole states. The magenta and blue vertical lines depict the calculated transition rates for the ${ }^{1} \Sigma_{g},-$ and ${ }^{3} \Sigma_{u},-$ double core hole states, respectively. Calculated transition energies have been downshifted by $2.3 \mathrm{eV}$. The experimental spectrum was taken from Ref. [91] (black errorbars). The calculated transitions originating from the ${ }^{1} \Sigma_{g},-$ double core hole state have been convoluted with a Gaussian function with $3.5 \mathrm{eV}$ FWHM (red line). 


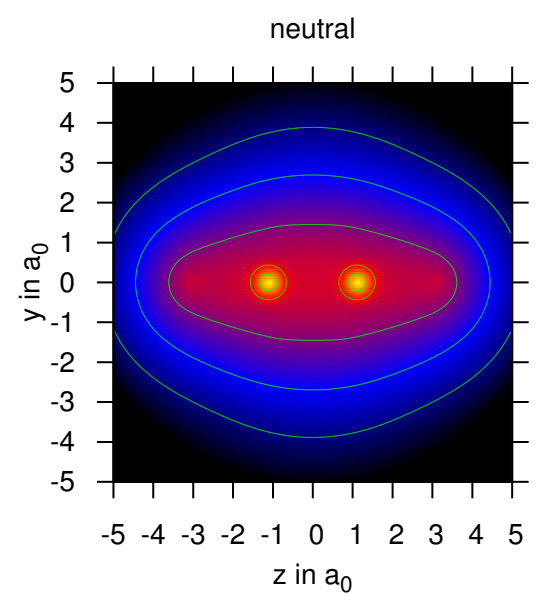

double core hole (two-site)

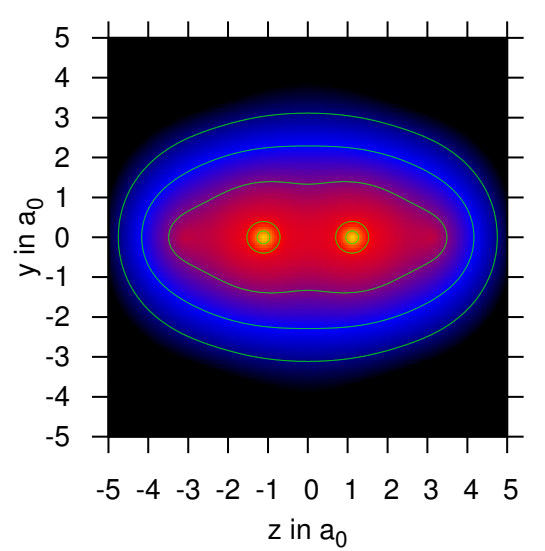

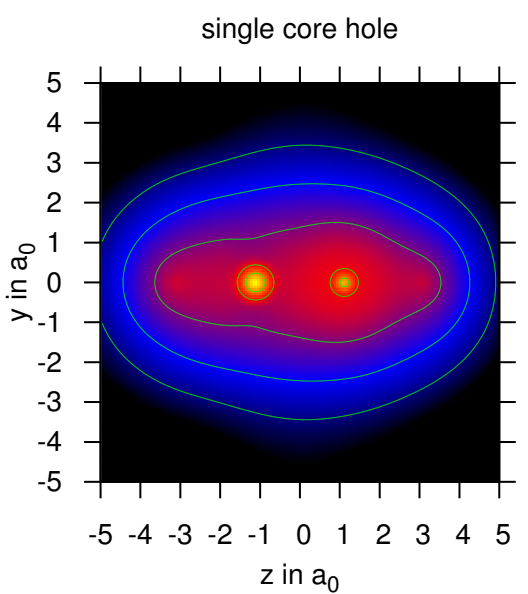

double core hole (single-site)

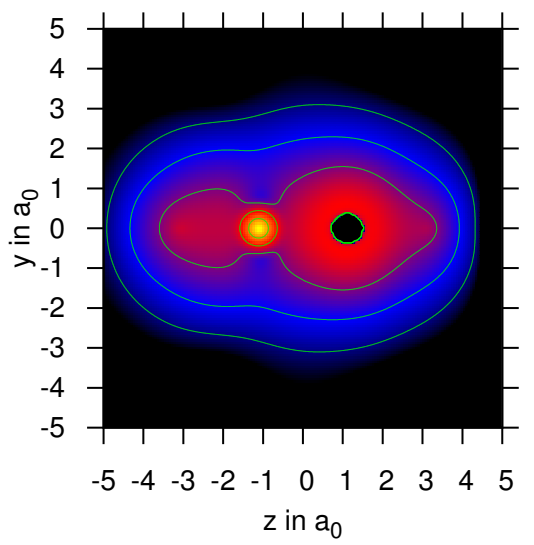

Figure 3.13: Planar cut through the electron density of acetylene $\left(\mathrm{C}_{2} \mathrm{H}_{2}\right)$ containing the molecular axis for the neutral, single, and double core ionized state (both on a single nuclear site and on two nuclear sites). Green lines denote the electron density contour lines. For each core hole state (single and double core holes) the electron density is deformed due to screening effects. 


\section{Results}

\subsubsection{Discussion \& Outlook}

In this section, I have applied the developed approach to the single and double core hole Auger decay in acetylene. Auger electron spectra and total decay rates were calculated for all types of single and double core holes. Calculated K-LL Auger transitions agree well with previously calculated 21] and experimentally determined values [13, 55, 57. For KK-KLL Auger transitions, the calculated spectra are in good agreement with those from experiments 63. Previous calculations addressing the KK-KLL Auger decay in molecules with two K-shells 91, 92 were based on an electron population estimate of the two-electron integrals and do not allow any statement about the decay rate. The calculation performed here is therefore the first ab-initio calculation of decay rates for this type of Auger decay. Interestingly, the decay of a single-site core hole was found to be much faster than that of the two-site double core hole, which is explained by the much stronger valence electron rearrangement for the single-site core holes. This finding again reveals that the core hole screening mechanism has a strong impact on the Auger decay.

Further studies should extend these calculations to ethane $\left(\mathrm{C}_{2} \mathrm{H}_{6}\right)$ and ethylene $\left(\mathrm{C}_{2} \mathrm{H}_{4}\right)$ to investigate a possible systematic influence of the electronic valence structure. Also, the dependence on the carbon-carbon bond length should be addressed. It was pointed out before [17] that the energies of two-site double core holes sensitively probe chemical environment. Thus, it can be speculated that the Auger spectrum of two-site double core holes also show a strong dependence on the molecular geometry. This way, Auger electron spectroscopy of two-site double core holes might provide a new tool for sensitive probing molecular geometries 90 . 


\section{Conclusion \& Outlook}

With the development of X-ray free electron lasers, the decay of molecular double core ionized states has become an active field of current research. One of the key processes relevant in this context is the Auger decay. In this thesis, I describe the development and implementation of a method to investigate Auger decay and ionization processes in small molecules after single and double core ionization. This method is based on configuration interaction calculations (CI) in combination with the single center approach to represent the electronic continuum. The accuracy of this approach was assessed in comparisons with previous experimental and theoretical studies. New results were obtained for the water molecule, where the the fast proton dynamics in relation to the core hole decay was investigated. Furthermore, I performed a study of absolute decay rates for double K-holes in first-row hydrides. These double K-holes were found to result in considerable rearrangements of valence electrons (core hole screening effects) that strongly enhance Auger decay rates. A relation to estimate these enhancements from a simple electron population analysis was derived. I also applied the developed approach to acetylene. Remarkably, the obtained Auger decay rates for acetylene were found to be substantially larger for double core holes located on the same nuclear site than for double core holes located on different nuclear sites.

Among these results, I consider most important that the decay of double K-holes in molecules is much faster than expected from single K-hole decay rates. As I have shown, this enhancement is a result of the relaxation of the molecular electronic structure during core ionization. The consequence of this finding is that significantly higher ionization rates in molecules exposed to intense X-ray radiation are achieved than was to be expected from atomic calculations or extrapolated from single K-hole decay rates.

A coherent diffractive imaging (CDI) experiment with single molecules necessarily involves multiple ionizations of core shells. The associated larger core hole decay rates help to faster refill core vacancies and thus effectively lead to a larger ionization rate. This finding may have considerable impact on the frustrated absorption mechanism, which is suggested to temporarily delay the radiation damage: As a double K-shell vacancy decays faster, the temporary induced X-ray transparency gained by the empty K-shell, 
lasts shorter. Thus, to rely on this mechanism, the pulse length of the X-ray beam has to be shorter, e.g., supposedly in the regime of the double K-hole lifetime. Future simulations of radiation damage in macro molecules could provide a more detailed insight in the consequences of this enhanced ionization rate and might predict more specific technical requirements for single molecule diffractive imaging. Whether this technical requirements can be met by the new European X-ray free electron laser at DESY 32] is not clear, yet.

Further implications for CDI experiments are connected to the electronic damage itself. Given the fact, that for one scattered photon about ten K-holes are created (which in Auger cascades will ionize the sample even more), the recorded electron density is unavoidably influenced by electronic relaxation (e.g., core hole screening) effects. Also, nuclear motion within the few femtoseconds of exposure cannot be neglected in general. As demonstrated here for a single water molecule, protons are immediately emitted from a biological sample. This phenomenon reduces the high charge within the molecule and thus might on a longer timescale delay further structural disintegration. A better understanding of these proton emittance combined with electronic relaxation effects may provide tools to account for radiation damage during reconstruction of the molecular geometry from obtained scattering data 74 .

Essential steps in investigating mechanisms of radiation damage in small molecules, which represent molecular components relevant for more complex bio-molecules, have been achieved in this work. To allow explicit statements for perspective CDI experiments, future work should aim at extending these calculations to further molecules. Current limitations are the computational cost and memory requirements for calculating the highly excited electronic states and the large number of angular quantum numbers $(\mathrm{lm})$, which needs to be considered. The latter requirement could be partially subdued by, for example, exploiting molecular symmetries more effectively. In particular, it would be feasible to include symmetry restrictions for the continuum electron, which would reduce the dimensionality of the solution vector of the continuum wave functions considerably. Also, the calculation cost could be reduced by using an advanced radial basis, e.g. radial splines or wavelets instead of a radial grid. Furthermore, the method presented here relies on a description of initial and final electronic states with identical sets of molecular orbitals and thus requires a large CI space (high truncation level). It is conceivable to adapt different sets of molecular orbitals for the initial and the final states in a way that the required CI truncation is reduced. This approach, however, would involve another unitary transformation for the calculation of the decay rates.

The ultimate aim is of course to study larger systems, e.g., complete macromolecules. 
To that effect a molecular component could in the calculation be embedded electrostatically into a larger chemical environment. Interestingly, this procedure would also allow to study the impact of a highly charged environment as expected in a CDI experiment. This charged chemical environment might influence on his part the local electronic structure, and thus modify Auger decay and ionization processes. First steps to investigate these effects have been made in recent single atom calculations [94].

Furthermore, an emitted electron may be slowed down or even trapped within a spatially confined region, which may also have an impact on Auger decay rates and allows the reverse process, dielectronic recombination. Also, future work may combine calculations of Auger decay rates with molecular dynamics simulations. This approach would allow to address the radiation damage in a macro molecule on longer timescales at which the nuclear displacement becomes more relevant. The computational procedure presented here could provide an essential component for such future studies. 


\section{Bibliography}

[1] M. Abramowitz and I. A. Stegun. Handbook of Mathematical Function with Formulas, Graphs, and Mathematical Tables. Vol. 55. National Bureau of Standards, Applied Mathematics Series, 1965.

[2] H. Ågren and H. Siegbahn. "Semi-internal correlation in the Auger-Electron spectrum of $\mathrm{H}_{2} \mathrm{O}$ ". Chemical Physics Letters 69, 424-429 (1980)

[3] E. Anderson, Z. Bai, C. Bischof, S. Blackford, J. Demmel, J. Dongarra, J. Du Croz, A. Greenbaum, S. Hammarling, A. McKenney, and D. Sorensen. LAPACK Users' Guide. Third. Philadelphia, PA: Society for Industrial and Applied Mathematics, 1999.

[4] V. Averbukh, U. Saalmann, and J. M. Rost. "Auger decay in the field of a positive charge". Physical Review A 85, 063405 (2012)

[5] Z. Bao, R. Fink, O. Travnikova, D. Ceolin, S. Svensson, and M. Piancastelli. "Detailed theoretical and experimental description of normal Auger decay in $\mathrm{O}_{2}$ ". Journal of Physics B (Atomic, Molecular and Optical Physics) 41, 125101 (2008)

[6] A. Barty, C. Caleman, A. Aquila, N. Timneanu, L. Lomb, T. A. White, J. Andreasson, D. Arnlund, S. Bajt, T. R. M. Barends, M. Barthelmess, M. J. Bogan, C. Bostedt, J. D. Bozek, R. Coffee, N. Coppola, J. Davidsson, D. P. DePonte, R. B. Doak, T. Ekeberg, V. Elser, S. W. Epp, B. Erk, H. Fleckenstein, L. Foucar, P. Fromme, H. Graafsma, L. Gumprecht, J. Hajdu, C. Y. Hampton, R. Hartmann, A. Hartmann, G. Hauser, H. Hirsemann, P. Holl, M. S. Hunter, L. Johansson, S. Kassemeyer, N. Kimmel, R. A. Kirian, M. Liang, F. R. N. C. Maia, E. Malmerberg, S. Marchesini, A. V. Martin, K. Nass, R. Neutze, C. Reich, D. Rolles, B. Rudek, A. Rudenko, H. Scott, I. Schlichting, J. Schulz, M. M. Seibert, R. L. Shoeman, R. G. Sierra, H. Soltau, J. C. H. Spence, F. Stellato, S. Stern, L. Strueder, J. Ullrich, X. Wang, G. Weidenspointner, U. Weierstall, C. B. Wunderer, and H. N. Chapman. "Self-terminating diffraction gates femtosecond X-ray nanocrystallography measurements". Nature Photonics 6, 35-40 (2012) 
[7] XCOM: Photon Cross Sections Database (version 1.5). M. Berger, J. Hubbell, S. Seltzer, J. Chang, J. Coursey, R. Sukumar, D. Zucker, and K. Olsen National Institute of Standards and Technology, Gaithersburg, MD. URL: http://www.ni st.gov/pml/data/xcom/(Nov. 2010)

[8] N. Berrah, L. Fang, B. Murphy, T. Osipov, K. Ueda, E. Kukk, R. Feifel, P. van der Meulen, P. Salen, H. T. Schmidt, R. D. Thomas, M. Larsson, R. Richter, K. C. Prince, J. D. Bozek, C. Bostedt, S.-i. Wada, M. N. Piancastelli, M. Tashiro, and M. Ehara. "Double-core-hole spectroscopy for chemical analysis with an intense X-ray femtosecond laser". Proceedings of the National Academy of Sciences of the United States of America 108, 16912-16915 (2011)

[9] C. P. Bhalla, N. O. Folland, and M. A. Hein. "Theoretical K-Shell Auger Rates, Transition Energies, and Fluorescence Yields for Multiply Ionized Neon". Physical Review A 8, 649-657 (1973)

[10] L. Biedenharn, J. Louck, and P. Carruthers. Angular Momentum in Quantum Physics: Theory and Application. Encyclopedia of Mathematics and its Applications Series. Addison-Wesley, Reading, 1981.

[11] J. S. Binkley, J. A. Pople, and W. J. Hehre. "Self-consistent molecular orbital methods. 21. Small split-valence basis sets for first-row elements". Journal of the American Chemical Society 102, 939-947 (1980)

[12] D. M. Bishop. "Single-Center Molecular Wave Functions". Advances in Quantum Chemistry 3, 25 -59 (1967)

[13] K. J. Børve, L. J. Sæthre, T. D. Thomas, T. X. Carroll, N. Berrah, J. D. Bozek, and E. Kukk. "Vibronic structure in the carbon $1 s$ photoelectron spectra of $\mathrm{HCCH}$ and DCCD". Physical Review A 63, 012506 (2000)

[14] R. Bulirsch and J. Stoer. Einführung in die Numerische Mathematik (Bd.I und II). Springer, 1978.

[15] C. F. Burmeister. "Primary Effects of X-ray and Photo-Absorption Induced Excitations in Biomolecules". PhD Thesis. Uni Göttingen, 2013.

[16] V. Carravetta and H. Ågren. "Stieltjes imaging method for molecular Auger transition rates - application to the Auger spectrum of water". Physical Review A 35, 1022-1032 (1987)

[17] L. S. Cederbaum, F. Tarantelli, A. Sgamellotti, and J. Schirmer. "On double vacancies in the core". The Journal of Chemical Physics 85, 6513-6523 (1986) 
[18] H. N. Chapman, P. Fromme, A. Barty, T. A. White, R. A. Kirian, A. Aquila, M. S. Hunter, J. Schulz, D. P. DePonte, U. Weierstall, R. B. Doak, F. R. N. C. Maia, A. V. Martin, I. Schlichting, L. Lomb, N. Coppola, R. L. Shoeman, S. W. Epp, R. Hartmann, D. Rolles, A. Rudenko, L. Foucar, N. Kimmel, G. Weidenspointner, P. Holl, M. Liang, M. Barthelmess, C. Caleman, S. Boutet, M. J. Bogan, J. Krzywinski, C. Bostedt, S. Bajt, L. Gumprecht, B. Rudek, B. Erk, C. Schmidt, A. Hoemke, C. Reich, D. Pietschner, L. Strueder, G. Hauser, H. Gorke, J. Ullrich, S. Herrmann, G. Schaller, F. Schopper, H. Soltau, K.-U. Kuehnel, M. Messerschmidt, J. D. Bozek, S. P. Hau-Riege, M. Frank, C. Y. Hampton, R. G. Sierra, D. Starodub, G. J. Williams, J. Hajdu, N. Timneanu, M. M. Seibert, J. Andreasson, A. Rocker, O. Joensson, M. Svenda, S. Stern, K. Nass, R. Andritschke, C.-D. Schroeter, F. Krasniqi, M. Bott, K. E. Schmidt, X. Wang, I. Grotjohann, J. M. Holton, T. R. M. Barends, R. Neutze, S. Marchesini, R. Fromme, S. Schorb, D. Rupp, M. Adolph, T. Gorkhover, I. Andersson, H. Hirsemann, G. Potdevin, H. Graafsma, B. Nilsson, and J. C. H. Spence. "Femtosecond X-ray protein nanocrystallography". Nature 470, 73-U81 (2011)

[19] M. H. Chen and B. Crasemann. "K-LL Auger transition probabilities for elements with low and intermediate atomic numbers". Physical Review A 8, 7-13 (1973)

[20] C. Cohen-Tannoudji, J. Dupont-Roc, and G. Grynberg. Atom-Photon Interactions. Wiley Science Paperback Series. Wiley, 1998.

[21] R. Colle, D. Embriaco, M. Massini, S. Simonucci, and S. Taioli. "Ab initio calculation of the normal Auger spectrum of C $2 \mathrm{H}$ 2". Journal of Physics B: Atomic, Molecular and Optical Physics 37, 1237 (2004)

[22] R. Colle, D. Embriaco, M. Massini, S. Simonucci, and S. Taioli. "Auger-electron angular distributions calculated without the two-step approximation: Calculation of angle-resolved resonant Auger spectra of $\mathrm{C}_{2} \mathrm{H}_{2}$ ". Physical Review A 70, 042708 (2004)

[23] M. Coville and T. D. Thomas. "Molecular effects on inner-shell lifetimes: Possible test of the one-center model of Auger decay". Physical Review A 43, 6053-6056 (1991)

[24] T. D. Crawford, C. D. Sherrill, E. F. Valeev, J. T. Fermann, R. A. King, M. L. Leininger, S. T. Brown, C. L. Janssen, E. T. Seidl, J. P. Kenny, and W. D. Allen. "PSI3: An open-source ab initio electronic structure package". Journal of Computational Chemistry 28, 1610-1616 (2007) 
[25] J. P. Cryan, J. M. Glownia, J. Andreasson, A. Belkacem, N. Berrah, C. I. Blaga, C. Bostedt, J. Bozek, C. Buth, L. F. DiMauro, L. Fang, O. Gessner, M. Guehr, J. Hajdu, M. P. Hertlein, M. Hoener, O. Kornilov, J. P. Marangos, A. M. March, B. K. McFarland, H. Merdji, V. S. Petrović, C. Raman, D. Ray, D. Reis, F. Tarantelli, M. Trigo, J. L. White, W. White, L. Young, P. H. Bucksbaum, and R. N. Coffee. "Auger Electron Angular Distribution of Double Core-Hole States in the Molecular Reference Frame". Physical Review Letters 105, 083004 (2010)

[26] P. Demekhin, D. Omel'yanenko, B. Lagutin, V. Sukhorukov, L. Werner, A. Ehresmann, K.-H. Schartner, and H. Schmoranzer. "Investigation of photoionization and photodissociation of an oxygen molecule by the method of coupled differential equations". Optics and Spectroscopy 102, 318-329 (2007)

[27] P. Demekhin, A. Ehresmann, and V. Sukhorukov. "Single center method: A computational tool for ionization and electronic excitation studies of molecules." The Journal of Chemical Physics 134, 024113 (2011)

[28] J. W. Demmel, N. J. Higham, and R. S. Schreiber. "Stability of block LU factorization". Numerical Linear Algebra with Applications 2, 173-190 (1995)

[29] G. Doumy, C. Roedig, S.-K. Son, C. I. Blaga, A. D. DiChiara, R. Santra, N. Berrah, C. Bostedt, J. D. Bozek, P. H. Bucksbaum, J. P. Cryan, L. Fang, S. Ghimire, J. M. Glownia, M. Hoener, E. P. Kanter, B. Krässig, M. Kuebel, M. Messerschmidt, G. G. Paulus, D. A. Reis, N. Rohringer, L. Young, P. Agostini, and L. F. DiMauro. "Nonlinear Atomic Response to Intense Ultrashort X-Rays". Physical Review Letters 106, 083002 (2011)

[30] R. W. Dunford, S. H. Southworth, D. Ray, E. P. Kanter, B. Krässig, L. Young, D. A. Arms, E. M. Dufresne, D. A. Walko, O. Vendrell, S.-K. Son, and R. Santra. "Evidence for interatomic Coulombic decay in Xe K-shell-vacancy decay of $\mathrm{XeF}_{2}$ ". Physical Review A 86, 033401 (2012)

[31] S. Dutta and H. M. Berman. "Large Macromolecular Complexes in the Protein Data Bank: A Status Report". Structure 13, 381 -388 (2005)

[32] Photon beam properties at the European XFEL. E.A. Schneidmiller and M.V. Yurkov Technical Report URL: https://docs.xfel.eu/alfresco/d/a/workspace/ SpacesStore / 32150a06 - 3b16 - 4c44 - 8ea5 - b1741220cdc2 / TR - 2011 - 006 _ Photon_Beam_Properties.pdf( 2011)

[33] B. Erk, D. Rolles, L. Foucar, B. Rudek, S. W. Epp, M. Cryle, C. Bostedt, S. Schorb, J. Bozek, A. Rouzee, A. Hundertmark, T. Marchenko, M. Simon, F. Filsinger, L. Christensen, S. De, S. Trippel, J. Küpper, H. Stapelfeldt, S. Wada, K. Ueda, M. Swiggers, M. Messerschmidt, C. D. Schröter, R. Moshammer, I. Schlicht- 
ing, J. Ullrich, and A. Rudenko. "Ultrafast Charge Rearrangement and Nuclear Dynamics upon Inner-Shell Multiple Ionization of Small Polyatomic Molecules". Physical Review Letters 110, 053003 (2013)

[34] E. Fick. Einführung in die Grundlagen der Quantentheorie. AKA-Lehrbuch. Leipzig: Akademische Verlagsgesellschaft Geest \& Portig, 1981.

[35] W. Friedrich, P. Knipping, and M. Laue. "Interferenzerscheinungen bei Röntgenstrahlen". Annalen der Physik 346, 971-988 (1913)

[36] Gaussian 09 Revision A.02. M. J. Frisch, G. W. Trucks, H. B. Schlegel, G. E. Scuseria, M. A. Robb, J. R. Cheeseman, G. Scalmani, V. Barone, B. Mennucci, G. A. Petersson, H. Nakatsuji, M. Caricato, X. Li, H. P. Hratchian, A. F. Izmaylov, J. Bloino, G. Zheng, J. L. Sonnenberg, M. Hada, M. Ehara, K. Toyota, R. Fukuda, J. Hasegawa, M. Ishida, T. Nakajima, Y. Honda, O. Kitao, H. Nakai, T. Vreven, J. A. Montgomery Jr., J. E. Peralta, F. Ogliaro, M. Bearpark, J. J. Heyd, E. Brothers, K. N. Kudin, V. N. Staroverov, R. Kobayashi, J. Normand, K. Raghavachari, A. Rendell, J. C. Burant, S. S. Iyengar, J. Tomasi, M. Cossi, N. Rega, J. M. Millam, M. Klene, J. E. Knox, J. B. Cross, V. Bakken, C. Adamo, J. Jaramillo, R. Gomperts, R. E. Stratmann, O. Yazyev, A. J. Austin, R. Cammi, C. Pomelli, J. W. Ochterski, R. L. Martin, K. Morokuma, V. G. Zakrzewski, G. A. Voth, P. Salvador, J. J. Dannenberg, S. Dapprich, A. D. Daniels, O. Farkas, J. B. Foresman, J. V. Ortiz, J. Cioslowski, and D. J. Fox Gaussian Inc. Wallingford CT 2009 ( 2009)

[37] K. J. Gaffney and H. N. Chapman. "Imaging atomic structure and dynamics with ultrafast X-ray scattering". Science 316, 1444-1448 (2007)

[38] Gnu Scientific Library: Reference Manual. M. Galassi, J. Davies, J. Theiler, B. Gough, G. Jungman, M. Booth, and F. Rossi URL: http://www.gnu.org/soft ware/gsl/manual/( 2003)

[39] R. Gaspar. "Über eine Approximation des Hartreefockschen Potentials durch eine universelle Potentialfunktion". Acta Physica Academiae Scientiarum Hungaricae 3, 263-286 (1954)

[40] G. Geloni, E. Saldin, L. Samoylova, E. Schneidmiller, H. Sinn, T. Tschentscher, and M. Yurkov. "Coherence properties of the European XFEL". New Journal of Physics 12, 035021 (2010)

[41] C. Gnodtke, U. Saalmann, and J. M. Rost. "Ionization and charge migration through strong internal fields in clusters exposed to intense x-ray pulses". Physical Review A 79, 041201 (2009) 
[42] Göppert-Mayer, Maria. "Über Elementarakte mit zwei Quantensprüngen". Annalen der Physik 401, 273-294 (1931)

[43] O. Gunnarsson and K. Schönhammer. "Dynamical theory of Auger processes". Physical Review B 22, 3710-3733 (1980)

[44] S. Hau-Riege. High-Intensity X-rays - Interaction with Matter: Processes in Plasmas, Clusters, Molecules and Solids. Wiley, 2012.

[45] S. P. Hau-Riege, R. A. London, G. Huldt, and H. N. Chapman. "Pulse requirements for x-ray diffraction imaging of single biological molecules". Physical Review E 71, 061919 (2005)

[46] S. P. Hau-Riege, R. A. London, and A. Szoke. "Dynamics of biological molecules irradiated by short x-ray pulses". Physical Review E 69, 051906 (2004)

[47] T. Helgaker, J. Olsen, and P. Jorgensen. Molecular Electronic-Structure Theory. Wiley, 2002.

[48] M. Hoener, L. Fang, O. Kornilov, O. Gessner, S. T. Pratt, M. Guehr, E. P. Kanter, C. Blaga, C. Bostedt, J. D. Bozek, P. H. Bucksbaum, C. Buth, M. Chen, R. Coffee, J. Cryan, L. F. DiMauro, M. Glownia, E. Hosler, E. Kukk, S. R. Leone, B. McFarland, M. Messerschmidt, B. Murphy, V. Petrovic, D. Rolles, and N. Berrah. "Ultraintense X-Ray Induced Ionization, Dissociation, and Frustrated Absorption in Molecular Nitrogen". Physical Review Letters 104, (2010)

[49] H. H. Homeier and E. Steinborn. "Some properties of the coupling coefficients of real spherical harmonics and their relation to Gaunt coefficients". Journal of Molecular Structure: THEOCHEM 368, 31 -37 (1996)

[50] L. Inhester, C. F. Burmeister, G. Groenhof, and H. Grubmüller. "Auger spectrum of a water molecule after single and double core ionization". The Journal of Chemical Physics 136, 144304 (2012)

[51] L. Inhester, G. Groenhof, and H. Grubmüller. "Core hole screening and decay rates of double core ionized first row hydrides". The Journal of Chemical Physics 138, 164304 (2013)

[52] Z. Jurek and G. Faigel. "The effect of inhomogenities on single-molecule imaging by hard XFEL pulses". Europhysics Letters 86, 68003 (2009)

[53] Z. Jurek and G. Faigel. "The effect of tamper layer on the explosion dynamics of atom clusters". The European Physical Journal D - Atomic, Molecular, Optical and Plasma Physics 50, 35-43 (2008) 
[54] Z. Jurek, G. Faigel, and M. Tegze. "Dynamics in a cluster under the influence of intense femtosecond hard X-ray pulses". The European Physical Journal D Atomic, Molecular, Optical and Plasma Physics 29, 217-229 (2004)

[55] B. Kempgens, H. Köppel, A. Kivimäki, M. Neeb, L. S. Cederbaum, and A. M. Bradshaw. "Core Level Energy Splitting in the C $1 s$ Photoelectron Spectrum of $\mathrm{C}_{2} \mathrm{H}_{2}$ ". Physical Review Letters 79, 3617-3620 (1997)

[56] J. Kendrew, G. Bodo, H. Dintzis, R. Parrish, and H. Wyckoff. "A Three-Dimensional Model of the Myoglobin Molecule Obtained by X-Ray Analysis". Nature 181, 662$666(1958)$

[57] A. Kivimäki, M. Neeb, B. Kempgens, H. M. Köppe, K. Maier, and A. M. Bradshaw. "Angle-resolved Auger spectra of the $\mathrm{C}_{2} \mathrm{H}_{2}$ molecule". Journal of Physics B: Atomic, Molecular and Optical Physics 30, 4279 (1997)

[58] P. Kolorenč and V. Averbukh. "K-shell Auger lifetime variation in doubly ionized Ne and first row hydrides". The Journal of Chemical Physics 135, 134314 (2011)

[59] P. Kolorenč, V. Averbukh, K. Gokhberg, and L. S. Cederbaum. "Ab initio calculation of interatomic decay rates of excited doubly ionized states in clusters". The Journal of Chemical Physics 129, 244102 (2008)

[60] M. O. Krause. "Atomic radiative and radiationless yields for K and L shells". Journal of Physical and Chemical Reference Data 8, 307-327 (1979)

[61] N. V. Kryzhevoi, R. Santra, and L. S. Cederbaum. "Inner-shell single and double ionization potentials of aminophenol isomers". The Journal of Chemical Physics 135, $084302(2011)$

[62] N. V. Kryzhevoi, M. Tashiro, M. Ehara, and L. S. Cederbaum. "Interatomic relaxation effects in double core ionization of chain molecules". The Journal of Chemical Physics 137, 154316 (2012)

[63] P. Lablanquie, T. P. Grozdanov, M. Žitnik, S. Carniato, P. Selles, L. Andric, J. Palaudoux, F. Penent, H. Iwayama, E. Shigemasa, Y. Hikosaka, K. Soejima, M. Nakano, I. H. Suzuki, and K. Ito. "Evidence of Single-Photon Two-Site Core Double Ionization of $\mathrm{C}_{2} \mathrm{H}_{2}$ Molecules". Physical Review Letters 107, 193004 (2011)

[64] S. Lavrent'ev, B. Lagutin, M. Vasil'eva, and V. Sukhorukov. "Photoionization of valence shells of neon-like molecules". Soviet Journal of Chemical Physics 7, 27280 (1990)

[65] R. B. Lehoucq, D. C. Sorensen, and C. Yang. Arpack User's Guide: Solution of Large-Scale Eigenvalue Problems With Implicityly Restorted Arnoldi Methods (Software, Environments, Tools). Society for Industrial \& Applied Math, 1997. 
[66] M. Leväsalmi, H. Aksela, and S. Aksela. "Satellite Structure in the KLL Spectrum of Neon". Physica Scripta 1992, 119 (1992)

[67] L. Lomb, T. R. M. Barends, S. Kassemeyer, A. Aquila, S. W. Epp, B. Erk, L. Foucar, R. Hartmann, B. Rudek, D. Rolles, A. Rudenko, R. L. Shoeman, J. Andreasson, S. Bajt, M. Barthelmess, A. Barty, M. J. Bogan, C. Bostedt, J. D. Bozek, C. Caleman, R. Coffee, N. Coppola, D. P. DePonte, R. B. Doak, T. Ekeberg, H. Fleckenstein, P. Fromme, M. Gebhardt, H. Graafsma, L. Gumprecht, C. Y. Hampton, A. Hartmann, G. Hauser, H. Hirsemann, P. Holl, J. M. Holton, M. S. Hunter, W. Kabsch, N. Kimmel, R. A. Kirian, M. Liang, F. R. N. C. Maia, A. Meinhart, S. Marchesini, A. V. Martin, K. Nass, C. Reich, J. Schulz, M. M. Seibert, R. Sierra, H. Soltau, J. C. H. Spence, J. Steinbrener, F. Stellato, S. Stern, N. Timneanu, X. Wang, G. Weidenspointner, U. Weierstall, T. A. White, C. Wunderer, H. N. Chapman, J. Ullrich, L. Strüder, and I. Schlichting. "Radiation damage in protein serial femtosecond crystallography using an x-ray free-electron laser". Physical Review $B$ 84, 214111 (2011)

[68] U. Lorenz, N. M. Kabachnik, E. Weckert, and I. A. Vartanyants. "Impact of ultrafast electronic damage in single-particle x-ray imaging experiments". Physical Review E 86, 051911 (2012)

[69] M. Mitani, O. Takahashi, K. Saito, and S. Iwata. "Theoretical molecular Auger spectra with electron population analysis". Journal of Electron Spectroscopy and Related Phenomena 128, 103-117 (2003)

[70] W. Moddeman, T. Carlson, M. Krause, B. Pullen, W. Bull, and Schweitz.GK. "Determination of K-LL Auger spectra of $\mathrm{N}_{2}, \mathrm{O}_{2}, \mathrm{CO}, \mathrm{NO}, \mathrm{H}_{2} \mathrm{O}$, and CO". The Journal of Chemical Physics 55, 2317 (1971)

[71] R. Neutze, R. Wouts, D. van der Spoel, E. Weckert, and J. Hajdu. "Potential for biomolecular imaging with femtosecond X-ray pulses". Nature 406, 752-757 (2000)

[72] J. Niskanen, P. Norman, H. Aksela, and H. Ågren. "Relativistic contributions to single and double core electron ionization energies of noble gases". The Journal of Chemical Physics 135, 054310 (2011)

[73] R. Pauncz. Spin eigenfunctions: construction and use. Plenum Press New York, 1979.

[74] H. M. Quiney and K. A. Nugent. "Biomolecular imaging and electronic damage using X-ray free-electron lasers". Nature Physics 7, 142 (2011) 
[75] J. Rasch and A. C. H. Yu. "Efficient Storage Scheme for Precalculated Wigner 3j, 6j and Gaunt Coefficients". Society for Industrial and Applied Mathematics Journal on Scientific Computing 25, 1416-1428 (2003)

[76] L. Redecke, K. Nass, D. P. DePonte, T. A. White, D. Rehders, A. Barty, F. Stellato, M. Liang, T. R. Barends, S. Boutet, G. J. Williams, M. Messerschmidt, M. M. Seibert, A. Aquila, D. Arnlund, S. Bajt, T. Barth, M. J. Bogan, C. Caleman, T.-C. Chao, R. B. Doak, H. Fleckenstein, M. Frank, R. Fromme, L. Galli, I. Grotjohann, M. S. Hunter, L. C. Johansson, S. Kassemeyer, G. Katona, R. A. Kirian, R. Koopmann, C. Kupitz, L. Lomb, A. V. Martin, S. Mogk, R. Neutze, R. L. Shoeman, J. Steinbrener, N. Timneanu, D. Wang, U. Weierstall, N. A. Zatsepin, J. C. H. Spence, P. Fromme, I. Schlichting, M. Duszenko, C. Betzel, and H. N. Chapman. "Natively Inhibited Trypanosoma brucei Cathepsin B Structure Determined by Using an X-ray Laser". Science 339, 227-230 (2013)

[77] A. G. Redfield. "On the Theory of Relaxation Processes". IBM Journal of Research and Development 1, 19-31 (1957)

[78] Protein Database Holdings Report. Research Collaboratory for Structural Bioinformatics URL: http://www.rcsb.org/pdb/statistics/holdings.do/June 2013)

[79] N. Rohringer and R. Santra. "X-ray nonlinear optical processes using a selfamplified spontaneous emission free-electron laser". Physical Review A 76, 033416 (2007)

[80] B. Rudek, S.-K. Son, L. Foucar, S. W. Epp, B. Erk, R. Hartmann, M. Adolph, R. Andritschke, A. Aquila, N. Berrah, C. Bostedt, J. Bozek, N. Coppola, F. Filsinger, H. Gorke, T. Gorkhover, H. Graafsma, L. Gumprecht, A. Hartmann, G. Hauser, S. Herrmann, H. Hirsemann, P. Holl, A. Hoemke, L. Journel, C. Kaiser, N. Kimmel, F. Krasniqi, K.-U. Kuehnel, M. Matysek, M. Messerschmidt, D. Miesner, T. Moeller, R. Moshammer, K. Nagaya, B. Nilsson, G. Potdevin, D. Pietschner, C. Reich, D. Rupp, G. Schaller, I. Schlichting, C. Schmidt, F. Schopper, S. Schorb, C.-D. Schroeter, J. Schulz, M. Simon, H. Soltau, L. Strueder, K. Ueda, G. Weidenspointner, R. Santra, J. Ullrich, A. Rudenko, and D. Rolles. "Ultra-efficient ionization of heavy atoms by intense X-ray free-electron laser pulses". Nature Photonics 6, 858-865 (2012)

[81] J. Sakurai and S. F. Tuan. Modern quantum mechanics. Vol. 1. Benjamin/Cummings, 1985. 
[82] R. Santra, N. V. Kryzhevoi, and L. S. Cederbaum. "X-Ray Two-Photon Photoelectron Spectroscopy: A Theoretical Study of Inner-Shell Spectra of the Organic Para-Aminophenol Molecule". Physical Review Letters 103, 013002 (2009)

[83] Quantum many particle physics. Schönhammer, K. Lecture notes Uni Göttingen (2007)

[84] H. Siegbahn, L. Asplund, and P. Kelfve. "The Auger electron spectrum of water vapour". Chemical Physics Letters 35, 330 -335 (1975)

[85] S. H. Southworth, E. P. Kanter, B. Krässig, L. Young, G. B. Armen, J. C. Levin, D. L. Ederer, and M. H. Chen. "Double K-shell photoionization of neon". Physical Review A 67, 062712 (2003)

[86] V. L. Sukhorukov, V. F. Demekhin, V. A. Yavna, I. D. Petrov, L. A. Demekhina, and S. V. Lavrentiev. "Расчет многоэлэктронных корреляций в молекулах". Координационная Химия 9, 158 (1983)

[87] A. Szabo and N. S. Ostlund. Modern Quantum Chemistry. New York: McGraw Hill, 1982.

[88] O. Takahashi, M. Odelius, D. Nordlund, A. Nilsson, H. Bluhm, and L. Pettersson. "Auger decay calculations with core-hole excited-state molecular-dynamics simulations of water". The Journal of Chemical Physics 124, (2006)

[89] F. Tarantelli, A. Sgamellotti, L. S. Cederbaum, and J. Schirmer. "Theoretical investigation of many dicationic states and the Auger spectrum of benzene". The Journal of Chemical Physics 86, 2201-2206 (1987)

[90] M. Tashiro, M. Ehara, H. Fukuzawa, K. Ueda, C. Buth, N. V. Kryzhevoi, and L. S. Cederbaum. "Molecular double core hole electron spectroscopy for chemical analysis". The Journal of Chemical Physics 132, 184302 (2010)

[91] M. Tashiro, M. Nakano, M. Ehara, F. Penent, L. Andric, J. Palaudoux, K. Ito, Y. Hikosaka, N. Kouchi, and P. Lablanquie. "Auger decay of molecular double core-hole and its satellite states: Comparison of experiment and calculation". The Journal of Chemical Physics 137, 224306 (2012)

[92] M. Tashiro, K. Ueda, and M. Ehara. "Auger decay of molecular double core-hole state". The Journal of Chemical Physics 135, 154307 (2011)

[93] J. Taylor. Scattering theory: the quantum theory on nonrelativistic collisions. Mineola, New York: Dover Publications, 2006.

[94] R. Thiele, S.-K. Son, B. Ziaja, and R. Santra. "Effect of screening by external charges on the atomic orbitals and photoinduced processes within the HartreeFock-Slater atom". Physical Review A 86, 033411 (2012) 
[95] J. Thom H. Dunning. "Gaussian basis sets for use in correlated molecular calculations. I. The atoms boron through neon and hydrogen". The Journal of Chemical Physics 90, 1007-1023 (1989)

[96] M. Toutounji. "Mixed quantum-classical Redfield master equation". The Journal of Chemical Physics 123, 244102 (2005)

[97] J. M. Varah. "On the Solution of Block-Tridiagonal Systems Arising from Certain Finite-Difference Equations". Mathematics of Computation 26, pp. 859-868 (1972)

[98] T. R. Walsh, T. E. Meehan, and F. P. Larkins. "Prediction of molecular Auger rates using a statistical model". Journal of Physics B: Atomic, Molecular and Optical Physics 27, 2211 (1994)

[99] J. Watson and F. Crick. "Molecular Structure of Nucleic Acids: A Structure for Deoxyribose Nucleic Acid". Nature 171, 737-738 (1953)

[100] V. Yarzhemsky and A. Sgamellotti. "Auger rates of second-row atoms calculated by many-body perturbation theory". Journal of Electron Spectroscopy and Related Phenomena 125, 13 -24 (2002)

[101] J. Yeh and I. Lindau. "Atomic subshell photoionization cross sections and asymmetry parameters: $1 \leq Z \leq 103$ ". Atomic Data and Nuclear Data Tables 32, 1 $-155(1985)$

[102] L. Young, E. P. Kanter, B. Kraessig, Y. Li, A. M. March, S. T. Pratt, R. Santra, S. H. Southworth, N. Rohringer, L. F. DiMauro, G. Doumy, C. A. Roedig, N. Berrah, L. Fang, M. Hoener, P. H. Bucksbaum, J. P. Cryan, S. Ghimire, J. M. Glownia, D. A. Reis, J. D. Bozek, C. Bostedt, and M. Messerschmidt. "Femtosecond electronic response of atoms to ultra-intense X-rays". Nature 466, 56-U66 (2010)

[103] K. Zähringer, H. Meyer, and L. Cederbaum. "Molecular-scattering wave-functions for Auger decay-rates - The Auger spectrum of hydrogen-fluoride". Physical Review A 45, 318-328 (1992)

[104] B. Ziaja, H. N. Chapman, R. Santra, T. Laarmann, E. Weckert, C. Bostedt, and T. Möller. "Heterogeneous clusters as a model system for the study of ionization dynamics within tampered samples". Physical Review A 84, 033201 (2011) 


\section{Lebenslauf}

Ludger Thomas InHESTER, geboren am 03.03.1983 in Göttingen,

Staatsangehörigkeit: deutsch.

\section{Schulbildung}

1990-1994 Godehardschule I, Göttingen

1994-1996 Bonifatiusschule II, Göttingen

1996-2002 Theodor Heuss Gymnasium Göttingen, abgeschlossen mit allgemeiner Hochschulreife

\section{Zividienst}

2002-2003

Göttinger Werkstätten gGmbH

\section{Studium}

2003-2005

Grundstudium Universität Karlsruhe (TH)

2005-2009 Hauptstudium Universität Göttingen, Abschluss: Diplom Physik

April $2009 \quad$ Diplomarbeit "Full counting statistics in Mehrfach-Verbindungen von Quantendrähten" in der AG Schönhammer am Institut für theoretische Physik Göttingen

seit August 2009 wissenschaftlicher Mitarbeiter und Promotionsstudent am Max Planck Institut für biophysikalische Chemie

Göttingen, 03. Juli 2013 